\title{
SOLAR HEATING AND HOT WATER SYSTEM INSTALLED AT SOUTHEAST OF SALINE, UNIFIED SCHOOL DISTRICT 306, MENTOR, KANSAS
}

Prepared from documents furnished by

Wilson \& Company

Engineerings and Architects

Salina, Kansas 67401

Under DOE Contract EG-77-A-01-4077

Monitored by the

National Aeronautics and Space Administration

George C. Marshall Space Flight Center, Alabama 35812

For the U. S. Department of Energy

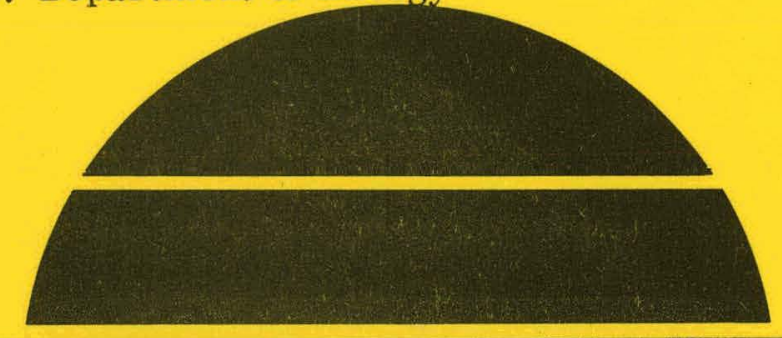

\section{U.S. Department of Energy}




\section{DISCLAIMER}

This report was prepared as an account of work sponsored by an agency of the United States Government. Neither the United States Government nor any agency Thereof, nor any of their employees, makes any warranty, express or implied, or assumes any legal liability or responsibility for the accuracy, completeness, or usefulness of any information, apparatus, product, or process disclosed, or represents that its use would not infringe privately owned rights. Reference herein to any specific commercial product, process, or service by trade name, trademark, manufacturer, or otherwise does not necessarily constitute or imply its endorsement, recommendation, or favoring by the United States Government or any agency thereof. The views and opinions of authors expressed herein do not necessarily state or reflect those of the United States Government or any agency thereof. 


\section{DISCLAIMER}

Portions of this document may be illegible in electronic image products. Images are produced from the best available original document. 
NOTICE

Th1s report was prepared to document work sponsored by the United States Government. Nelther the United States nor 1ts agents the United Srates Department of Energy, the United States Nat1onal Aeronaut1c8 and Space Adminlstration, nor any federal employees, nor any of their contractors, subcontractors or the1r employees, make any warranty, express or 1mpl1ed, or assume any legal liabllity or responsibility for the accuracy, completeness, or usefulness of any information, apparatus, product or process disclosed, or represent that 1 ts use would not infringe privately owned rights. 


\begin{tabular}{|c|c|}
\hline $\begin{array}{l}\text { 1. REPORT NO. } \\
\text { DOE/NASA CR-161271 }\end{array}$ & 3. RECIPIENT'S CATALOG NO. \\
\hline \multirow{2}{*}{$\begin{array}{l}\text { 4. TITLE AND SUBTITLE } \\
\text { Solar Heating and Hot Water System Installed at Southeast of } \\
\text { Saline, Unified School District } 306 \text {, Mentor, Kansas }\end{array}$} & $\begin{array}{l}\text { 5. REPORT DATE } \\
\text { July } 1979\end{array}$ \\
\hline & 6. PERFORMING ORGANIZATION CCIDE \\
\hline 7. AUTHOR(S) & 8. PERFORMING ORGANIZATION REPORT \# \\
\hline \multirow{3}{*}{$\begin{array}{l}\text { 9. PERFORMING ORGANIZATION NAME AND ADORESS } \\
\text { Wilson \& Company } \\
\text { Engineers \& Architects } \\
\text { Salina, Kansas }\end{array}$} & 10. WORK UNIT, NO. \\
\hline & $\begin{array}{l}\text { 11. CONTRACT OR GRANT NO. } \\
\text { DOE EG-77-A-01-4077 }\end{array}$ \\
\hline & 13. TYPE OF REPORI \& PERIOD COVERED \\
\hline \multirow{2}{*}{$\begin{array}{l}\text { 12. SPONSORING AGENCY NAME AND ADDRESS } \\
\text { National Aeronautics and Space Administration } \\
\text { Washington, D. C. } 20546\end{array}$} & Contractor Report \\
\hline & 14. SPONSORING AGENCY CODE \\
\hline
\end{tabular}

15. SUPPLEMENTARY NOTES

This work was done under the technical management of Mr. Lary Lawson, George C. Marshall Space Flight Center, Alabama.

16. ABSTRACT

A cooperative agreement was negotiated in April 1978 for the installation of a space and domestic hot water system at Southeast of Saline, Kansas Unified School District 306, Mentor, Kansas, in cooperation with the U. S. Department of Energy. The Project Manager for this effort was Mr. James Hall II, Wilson \& Co., Salina, Kansas.

The solar system was installed in a new building and was designed to provide 52 percent of the estimated annual space heating load and 84 percent of the estimated annual potable hot water requirement. The collectors are liquid flat plate, manufactured by Inter-Technology Corporation. They are ground-mounted and cover a total area of 5125 square feet. The system will provide supplemental heat for the school's closed-loop water-to-air heat pump system and domestic hot water. The storage medium is water inside steel tanks with a capacity of 11, 828 gallons for space heating and 1,600 gallons for domestic hot water.

This final report, which describes in considerable detail the solar heating facility, contains detailed drawings of the completed system. The facility was declared operational in September 1978, and has functioned successfully since.

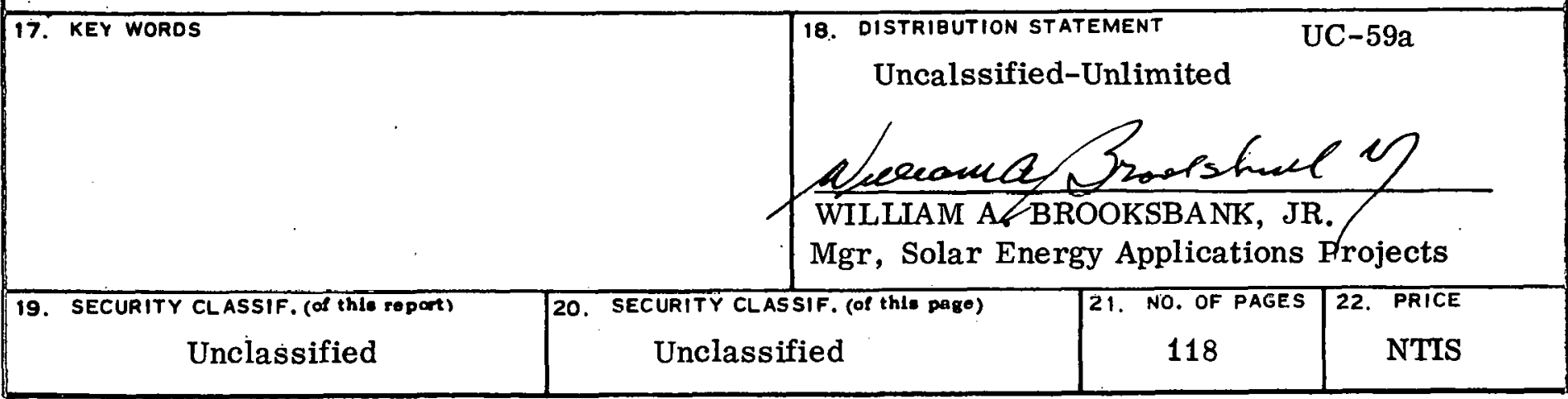


THIS PAGE

\section{WAS INTENTIONALLY LEFT BLANK}


Page No.

I. Description of Building and Contents

a. Building Description

b. Design Philosophy

c. Solar System Design Overview

(1) General Description

(2) Site Planning

(3) Major Components

II. Project Cost Data

III. Acceptance Test Plan Data 3

a. Mechanical $\quad 3$

b. Electrical

IV. Interim Performance Criteria Certification 5

V. Operation \& Control Logic 6

a. Thermal Storage Tank Heating 6

b. Domestic Water Heating 6

VI. Problems Encountered and Solutions 6

VII. Successful Components and Processes 7

VIII. Lessons Learned and Recommendations 7

Appendix A - As-Built Drawings

Appendix B - Parts Listing and Manufacturer's Literature

Appendix C - Supplemental Cost Data

Appendix D - Pictures

Appendix E - Supplemental Control, Maintenance and Testing Data

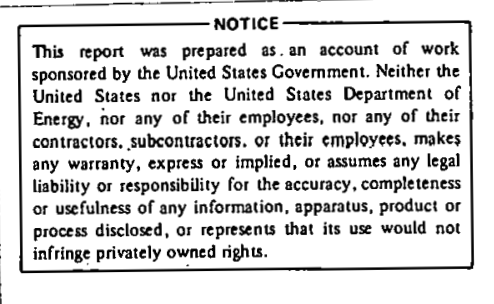




\section{DESCRIPTION OF BUILDING AND CONTENTS}

a. Building Description. The USD \#306 school building serves an estimated 750 students ranging in age from Kindergarten to 12th grade with approximately 140,000 s.f. of floor area. General building construction is metal frame with brick fascia. Low heat loss characteristics are incorporated into building construction by the use of double glazed windows with a low ratio of window to wall space and the generous use of batt insulation. Building thermal characteristics are typified by overall U-values of 0.05 and 0.07 for the walls and roof respectively. An aerial photograph including both school and collector field is shown on page 9.

Building heating consists of a closed loop water to air heat pump system with individual heat pumps in each classroom area, as well as roof-mounted heat pumps for the larger areas such as the gymnasium and the auditorium. The auxiliary heating system is all electric. The domestic water heating system consists of electric powered hot water generators which are supplemented by solar pre-heating.

The solar supplemental system was designed to contribute approximately 50 percent of the building's space heating needs and 80 percent of the domestic hot water needs for the year. Early estimates of the power costs savings to the school district are approximately $\$ 10,000-\$ 11,000$ per year.

b. Design Philosophy. In the early design stages of the facility, the school board authorized the design of a system with sufficient flexibility to operate either with or without solar supplemental heat. The solar collectors were to be iucluded only if a grant could be secured to pay for part of the solar installation. Accordingly, these provisions were included in the design for a future solar system. Construction was well underway before the grant application was approved to install the supplemental solar heating system. These provisions for future installation made it relatively easy to incorporate the new solar equipment into the existing building system.

c. Solar System Design Overview. The building heat pump system requires circulation water temperatures between $70^{\circ} \mathrm{F}$ and $95^{\circ} \mathrm{F}$. Because of the relatively low temperatures required the decision was made to use flat-plate collectors for solar energy collection. In comparison with high-temperature collection systems this resulted in lower overall costs, simpler design and greater operational efficiency.

(1) General Description. The entire solar supplemented building heating and cooling system, shown schematically on page 10, consists of four heat transfer loops. In the first loop a glycol/water solution is pumped from a field of solar collectors to the tube side of the solar loop heat exchanger. Water is pumped in the second loop from the shell side of the same heat exchanger to thermal storage tanks each equipped with auxiliary electric heaters required for solar system back-up. In the third loop, water is pumped from the thermal storage tanks to the shell side of a second heat exchanger used for domestic water preheat. Building space 
heating and cooling is performed in the fourth loop as water from the thermal storage tanks is pumped to the individual building heat pumps where heat is either pumped out of (room heating) or into (room cooling) the water source. Since each heat pump is capable of independently satisfying its individual load, the cooling of internal heat gains in one area of the building provides heat to other areas requiring heat via the central energy thermal storage tanks.

The physical location of major equipment items is in two areas, the mechanical equipment room and the collector field.

(2) Site Planning. The collector field is located on level ground approximately 120 feet due south of the southeast corner of the main school building. Overall field size is 154 feet by 70 feet with individual collectors facing directly south and tilted at an angle of $53^{\circ}$ fruil the horizontal, optimum for solar energy collection during the winter months.

The collectors are ground mounted due in part to the installation of collectors after the design of the building had been completed and to the belief that ground mounting would allow easier maintenance and better public view of the actual solar hardware and avoidance of roof leakage problems.

Internal headering between individual collectors allows for their arrangement in rows. A structural steel frame, embedded in concrete piers, was used to support each row.

A 4 feet high chain link fence surrounds the collector field at a distance of 10 feet from the field perimeter.

Supply and return piping mains for the collector field were run underground from the main to the field proper. All the piping within the field itself is above ground.

(3) Major Components. 'The tollowing are brlef descriptions of the major solar system components. (See Appendix B for manufacturer's litcrature.)

(a) The collectors are of the flat-plate type with single tempered glazing and black chromium absorber coating. Internal cross tubes and manifolds are copper and are arranged in a gated flow configuration. Aluminum absorber fins are mechanically bonded to the cross tubes.

type.

(b) The heat exchangers are water-to-water shell and tube

(c) Pumps for the circulation loop from the thermal energy storage tanks to the solar heat exchanger are base mounted, centrifugal type. All other pumps in the system are in-line centrifugal type. 
(d) The expansion tanks are ASME rated with gauge glass.

(e) The air separator is a pipeline-mounted steel tank with strainer. Tank inlet and outlet are tangentially-mounted.

(f) The three way flow diverting valve is made of cast iron with flanged ports. Two temperature sensitive control elements are internally mounted.

(g) The heat rejector consists of a finned-tube radiator and motorized fan mounted on a steel base.

II. PROJECT COST DATA

Consultants

$\underline{\text { Costs }}$

Wilson \& Company, Engineers \& Architects

Intertechnology Corp.

$\$ 15,635$

1,535

Contractors

Mechanical

Buckley, Inc.

163,700

Electrical

Electrical Equipment Co., Inc. 11,476

General

J.S. Frank Construction Co., Inc.

87,807

TOTAL CONSTRUCTION COSTS

$\$ 280,153$

DOE Funding

$\$ \underline{181,000}$

PROJECT COST TO OWNER

$\$ 99,153$

The project, as bid and built, had no cost overruns. However, moneys were not provided for monitoring instrumentation, which will be necessary if extensive performance data collection is ever desired.

\section{ACCEPTANCE TEST PLAN DATA}

Testing of the solar system, during construction and after, was handled by the contractors involved, under observation of a designated Wilson \& Company representative. The following are outlines of the testing performed.

a. Mechanical. With the exception of the solar collector arrays, system piping and components were hydrostatically tested at a pressure equal to that in the water main serving the building, approximately 55 psig. The collector arrays were hydrostatically tested at $30 \mathrm{psig}$. Each of these test pressures was held for a 24 hour period.

Al1 pump motors were tested for proper rpm, suction pressure, discharge pressure and motor current and voltage. 
b. Electrical. The completed wiring system was tested for open circuits, short circuits, and grounds. Load balancing was performed on the electrical distribution system to provide a balanced load on each phase.

The system became operational in September 1978. The collector to heat exchanger loop was first filled with water for testing and balancing under operating conditions. In October 1978 the water was drained and replaced with a solution of 50 percent propylene glycol and 50 percent water.

The temperature, flow rate and manual calculations that have been done indicate that the system is collecting thermal energy as predicted. Since no thermal energy recording instruments were included in the project detailed performance information cannot be provided. 
WILSON

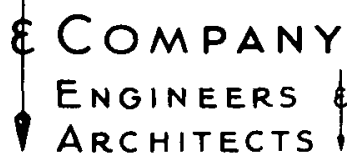

ENGINEERS

ARCHITECTS

PLANNERS

An Equal Opportwnity Employer

16 May 1979

Mr. Kenneth D. Sowell

Contracting officer

National Aeronautics \& Space Admin.

George C. Marshall Space Flight Center

Marsha11 Space Flight Center

Huntsville, AL 35812

Re: DOE Contract EG-77-A-01-4077

Interim Performance Criteria

Sir:

Consider this letter as our letter of certification that the solar heating system under discussion meets the Tnterim Performance Critcria, where said criteria are applicable.

WILSON \& COMPANY

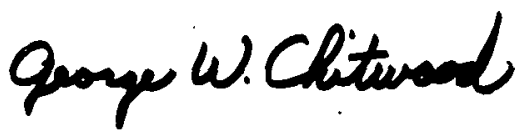

George W. Chitwood 
The solar system operation may be divided into two semi-independent control. sequences, thermal storage tank heating and domestic water heating. A schematic of the control system is shown in Appendix A drawing $\mathrm{M}-20$, Solar Heating System Schedules and Schematics.

a. Thermal Storage Tank Heating. Heating of the tank is accomplished by differential temperature control of the system pumps. When the collector plate temperature rises $20^{\circ} \mathrm{F}$ above the water storage temperature a temperature differential switch, TDS-1, will start the collector loop pumps, P-5, 6 and the storage loop pumps, P-3, 4. The pumps will continue to run until the temperature differential, as sensed by TDS-1, decreases to $5^{\circ} \mathrm{F}$.

If the differential has been reduced to $5^{\circ} \mathrm{F}$ but the collector temperature exceeds $190^{\circ} \mathrm{F}$ a temperature switch, TS-2, will override TDS-1 to keep the collector loop pumps running to provide circulation through the heat rejector. At $210^{\circ} \mathrm{F}$ an internal sensor starts the heat rejector fan.

b. Domestic Water Heating. Again healing of the tank is accompliahcd by differential temperature control of the system pumps. When the thermal storage tank temperature rises to $20^{\circ} \mathrm{F}$ above the domestic water tank temperature a temperature differential switch, TDS-3, will start the domestic water pre-heat pumps, $P-7,8,9,10$. The pumps will continue to run until the differential decreases to $5^{\circ} \mathrm{F}$.

When the domestic water tank temperature reaches $140^{\circ} \mathrm{F}$, regardless of the current differential temperature, a temperature switch, TS-4, will. override TDS-3 to stop the pumps.

\section{PROBLEMS ENCOUNTERED AND SOLUTIONS}

Cost escalation from the time the proposal was prepared to the time that the contracts were actually awarded was responsible for several changes aimed at keeping the project on budget without the sacrifice of any essential itcms. By deleting the permanent installation of standby pumps (these are stored in shipping crates in the equipment room), shifting from the use of base-mounted to in-line pumps and careful analysis and redesign of the collector structural support system, considerable cust savings were cffected.

One of the structural problems encountered was alignment of collectors on the mounting frame. This was solved through the use of slotted holes in the structural framing members allowing some "play" between collector and mounting frame.

The factory supplied pressure cap for the heat rejector was found to have too low a pressure rating. It was replaced with a higher rated cap.

One of the major problems encountered was that of the hose connections between adjacent collectors. The design of these hose connections proved to be extremely tight, allowing very little room to work. The collectors had to be modified in the field by cutting so that installation could 
actually be made. These hose connections, although satisfactory now, presented difficulty in securing a leak-free installation, and required considerable effort on the part of the mechanical contractor.

Problems were encountered with some of the solid-state control components and the use of unshielded control cables. Both. of these items have been replaced and are now operating satisfactorily.

Glass breakage of some of the collector cover plates occurred on the job. All were immediately replaced. Real-life testing of the tempered glass in a Kansas hail storm has not occurred yet, but the owner has secured insurance coverage for glass coverplate liability.

\section{SUCCESSFUL COMPONENTS OR PROCESSES}

The entire project is an overall success and works as it was intended, despite minor start-up problems. Some of the more successful design features which should be mentioned include the use of preinsulated jacketed steel pipe for the underground portion of the piping, tempered glass for the collector covers and metallic jacketing for exterior aboveground insulation. In erecting the structural steel support system, the general contractor used a laser type alignment device which proved helpful by making actual collector installation easier. As previously mentioned, the use of slotted adjustable bolt attachments for the interface between the structural steel frame and the collectors proved very helpful in collector installation.

One of the best design featues in the entire project was the use of internal headering between individual collectors. This minimized the connections between the collectors and the supply and return distribution mains and was extremely helpful in controlling the cost of the project. Calibrated balancing valves were used to adjust the flow at each bank of collectors and a reverse return type piping distribution system simplified the job of balancing flows to the collectors.

Incorporation of the future installation of solar supplemental heat into the original system design made the work of interconnecting after the system was well under construction relatively easy. Only minor modifications to piping were required.

\section{LESSONS LEARNED AND RECOMMENDATIONS}

On subsequent projects dealing with supplemental solar heating, the following changes would be desirable:

1. Spare glass coverplates should be ordered for the Owner to have in case of accidental breakage.

2. Brazed or soldered internal heater connections between solar collectors would be more desirable and less time consuming for field installation. 
3. From the standpoint of avoiding potential roof leakage problems, ground mounted collectors are preferable to roof mounting provided space is available.

4. The structural steel collector mounting system could probably be improved and installed in a less expensive fashion by incorporating something similar to a Uni-strut system.

5. The heat rejector radiator pressure rating should be high enough to avoid problems with pressure blow-off.

6. A permanent removable cover should be provided for covering of collectors and heat rejector when system is not used during summer months.

7. On any future project it would be advisable to include some simplc basic instrumentation for project monitoring to determine the extent of the solar contribution and its effectiveness. 


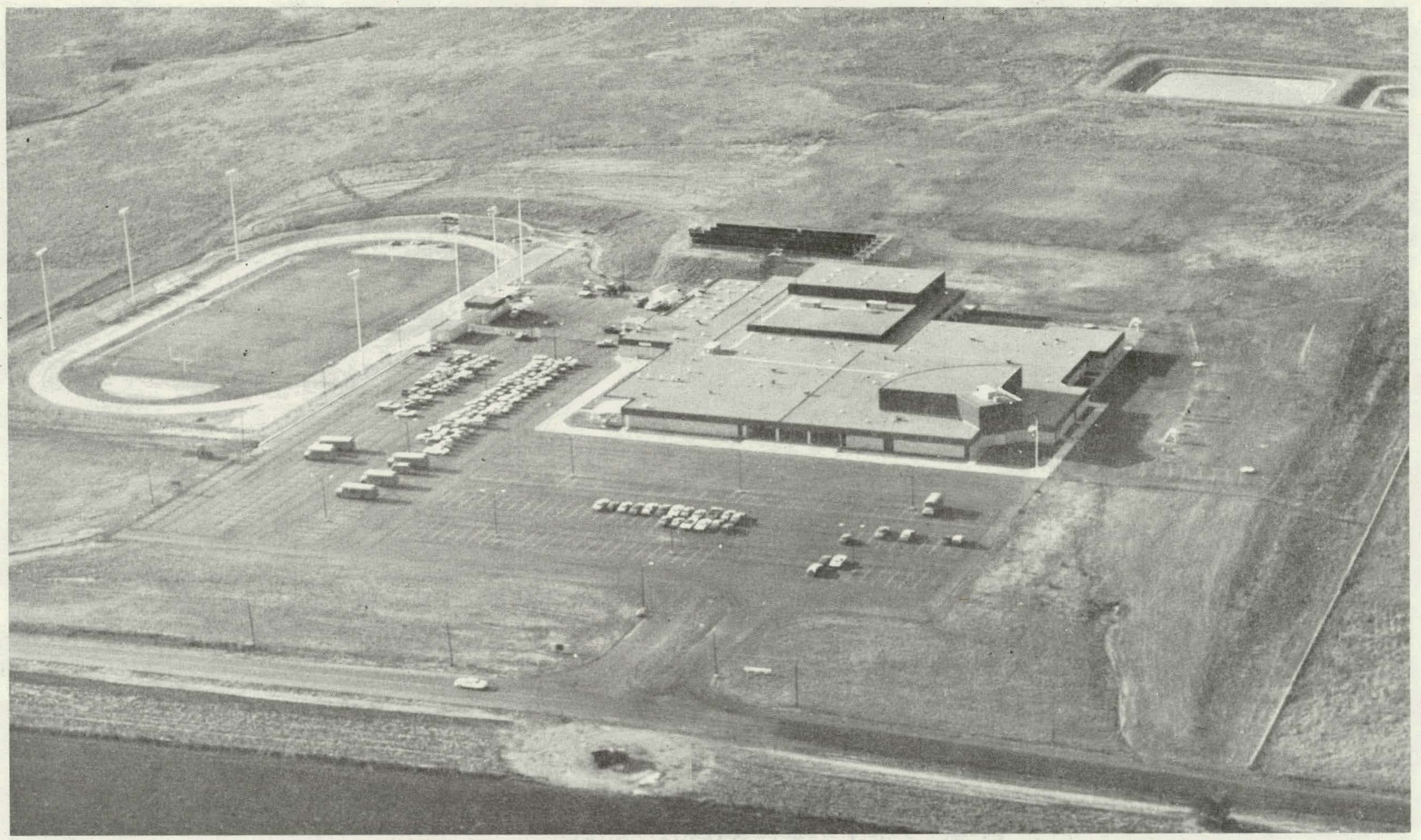

KANSAS UNIFIED SCHOOL DISTRICT 306

MENTOR, KANSAS

SCHOOL HEATING SOLAR DEMONSTRATION PROJECT

PON JSE-76-2 


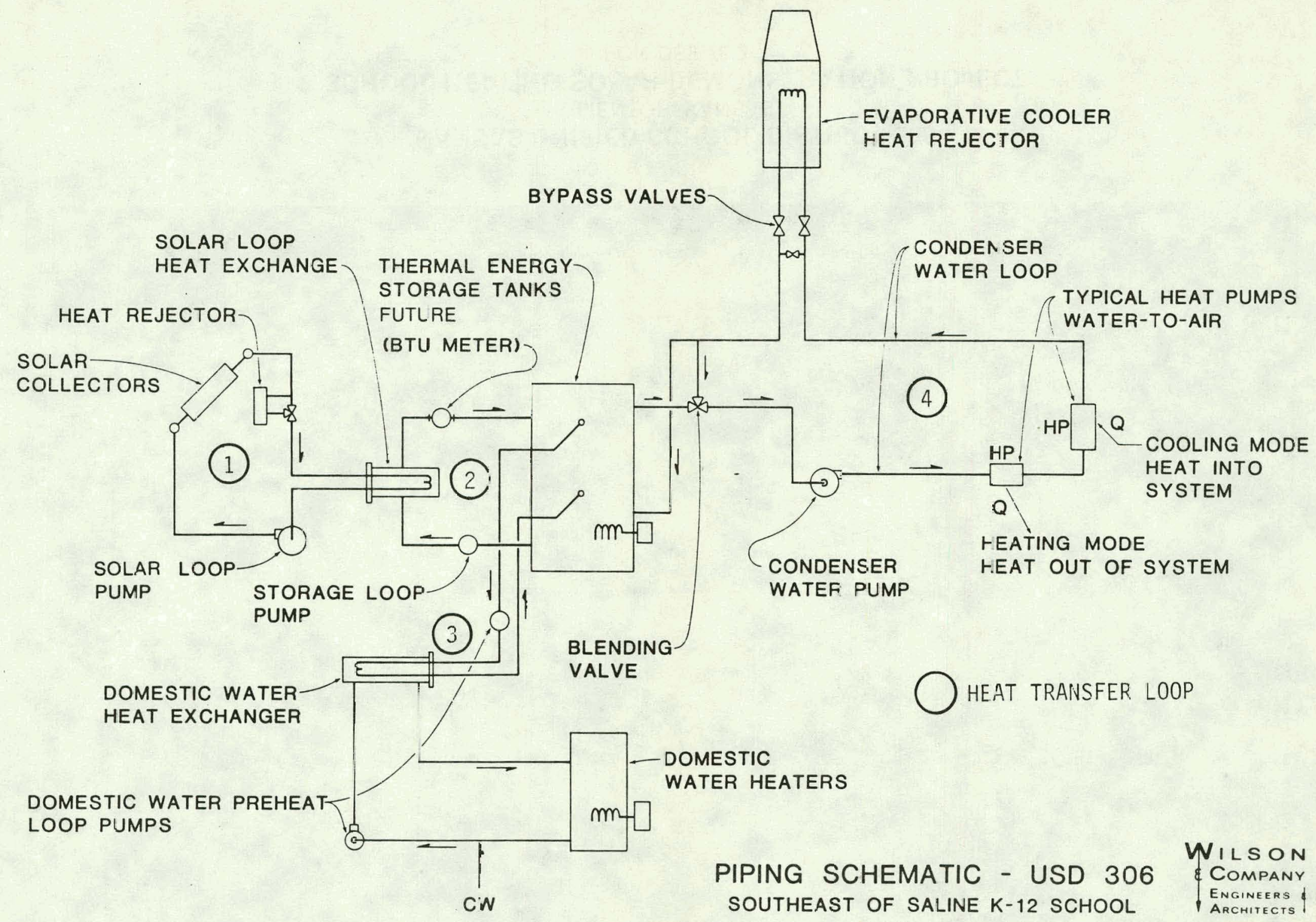




\section{APPENDIX A}

AS-BUILT DRAWINGS

$\begin{array}{ll}\text { A-1 } & \text { DRAWING } M-19 \\ \text { A-2 } & \text { DRAWING } M-20 \\ \text { A-3 } & \text { DRAWING M-15 } \\ \text { A-4 } & \text { DRAWING M-5 } \\ \text { A-5 } & \text { DRAWING } 1 \text { OF } 3 \\ \text { A-6 } & \text { DRAWING S-17 } \\ \text { A-7 } & \text { URAWING E-1 } \\ \text { A-8 } & \text { DRAWING E-4 } \\ \text { A-9 } & \text { DRAWING E-19 }\end{array}$

SOLAR COLLECTION INSTALLATION

SOLAR HEATING SYSTEM-SCHEDULES AND SCHEMATICS SOLAR COLLECTION SYSTEM LAYOUT

DOMESTIC HOT WATER SYSTEM LAYOUT

SITE PLAN

COLLECTOR MOUNT DETAILS

SITE ELECTRICAL LAYOUT

BUILDING ELECTRICAL SCHEMATIC

ELECTRICAL FLOOR PLAN 


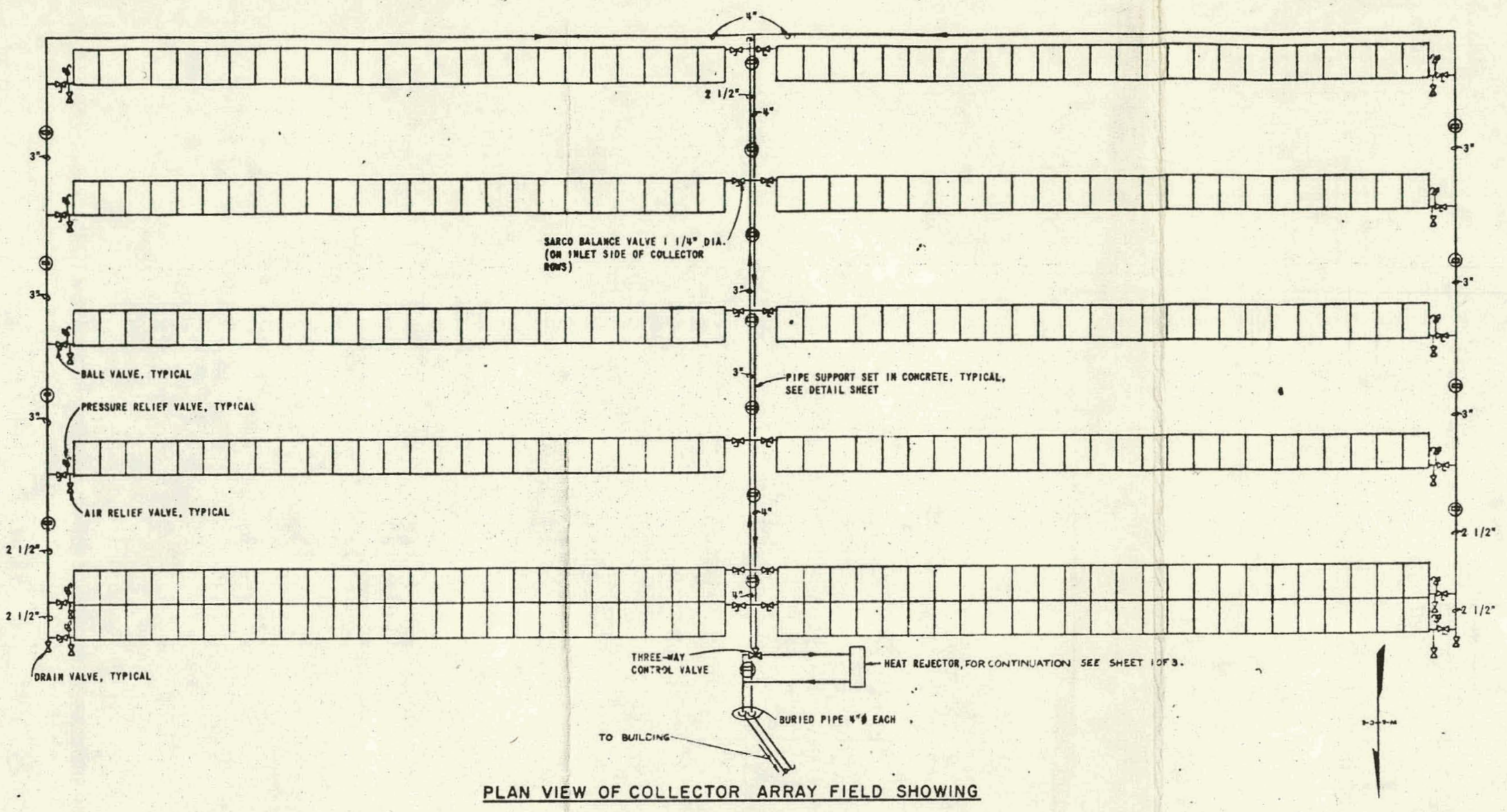

wises:

(1)

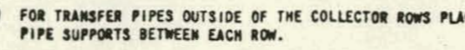

TRANSEER PIPING

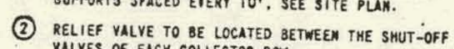
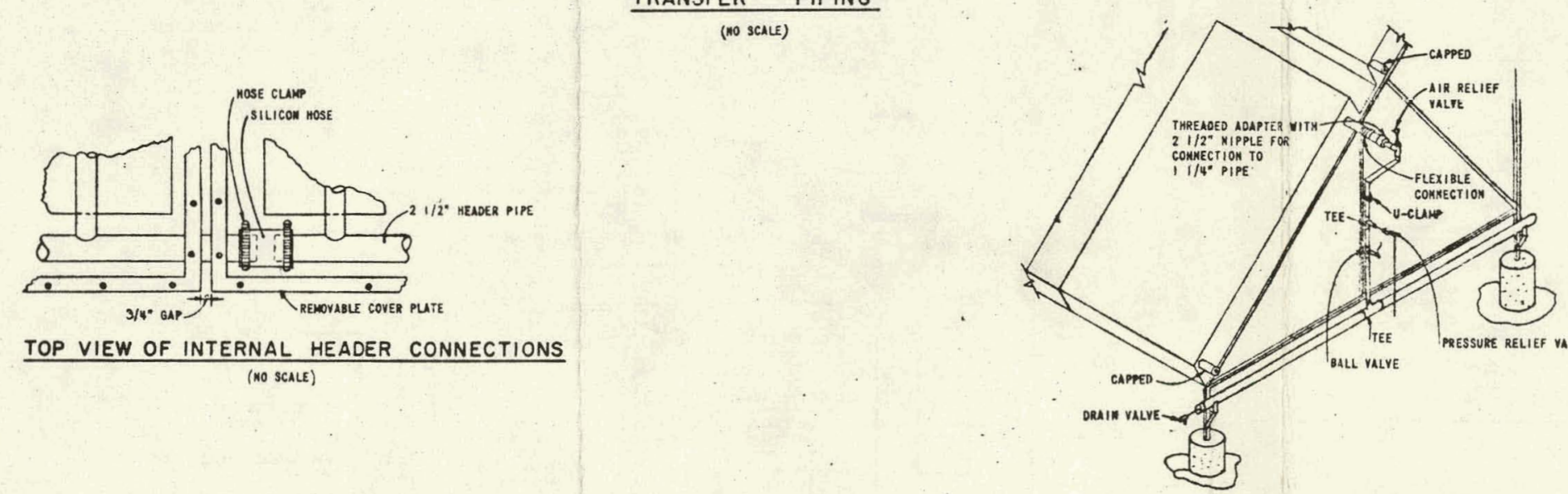

ISOMETRIC VIEW OF COLLECTOR END

OF ROW RETURN LINE SHOWING

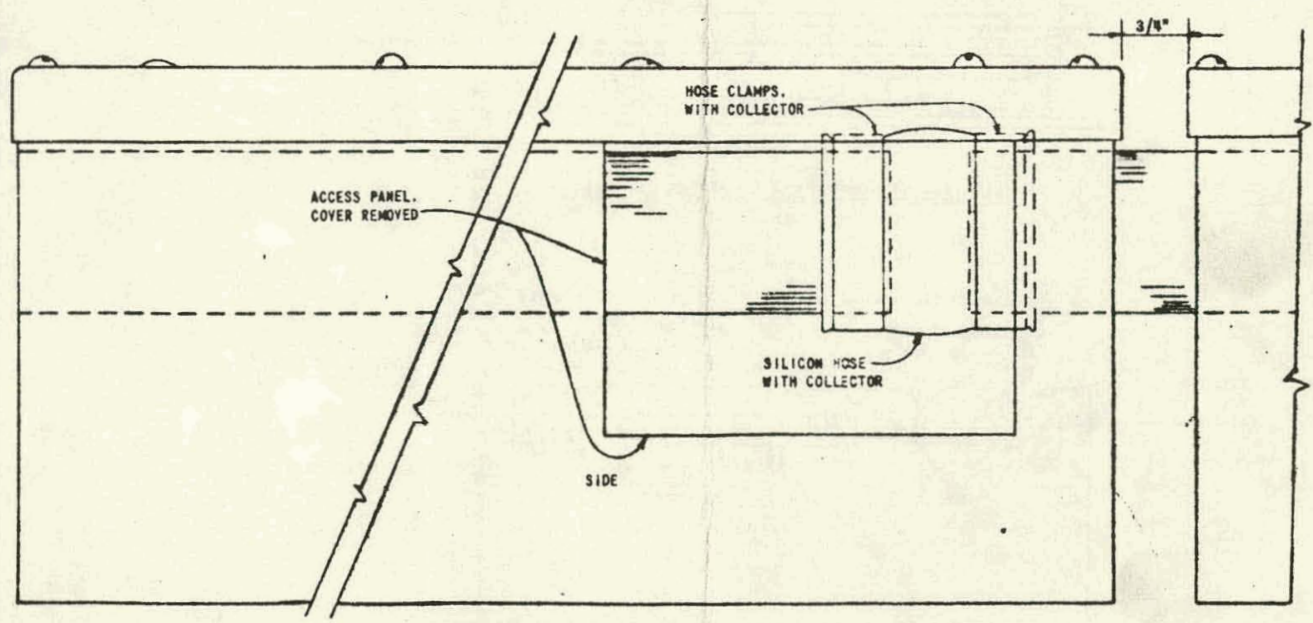

SIDE VIEW OF INTERNAL HEADER CONNECTIONS
T)

(1)

$\frac{\text { FITTINGS }}{\text { (os osale) }}$

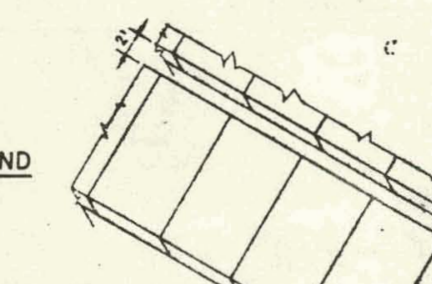

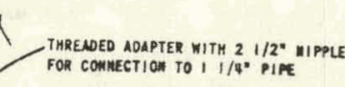

Q

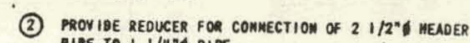

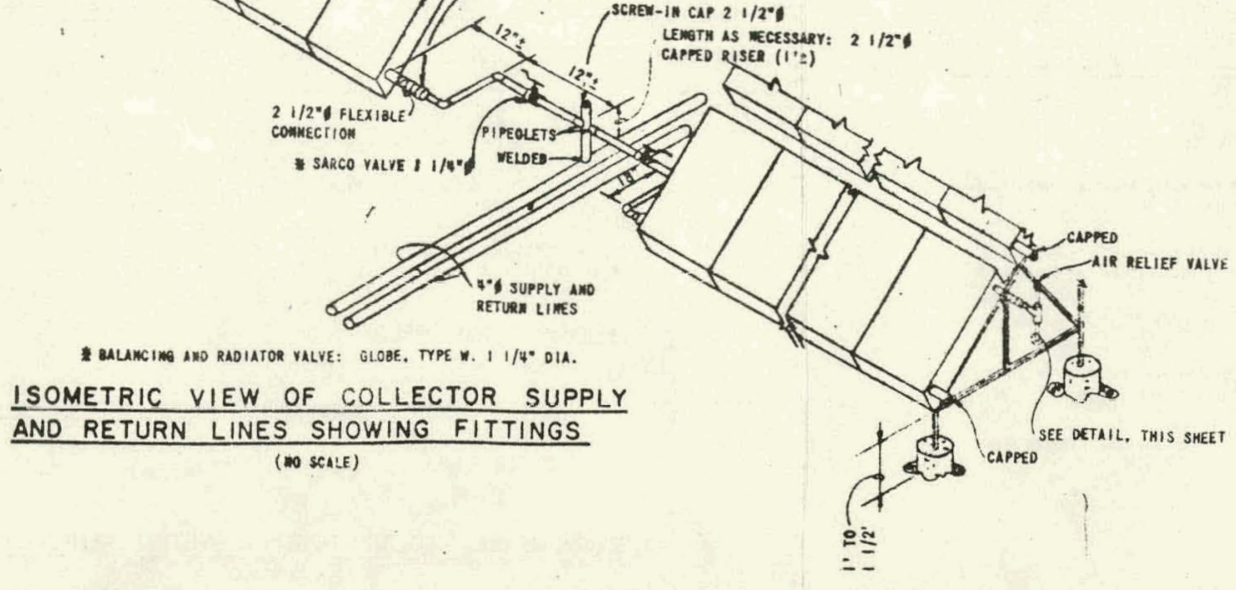




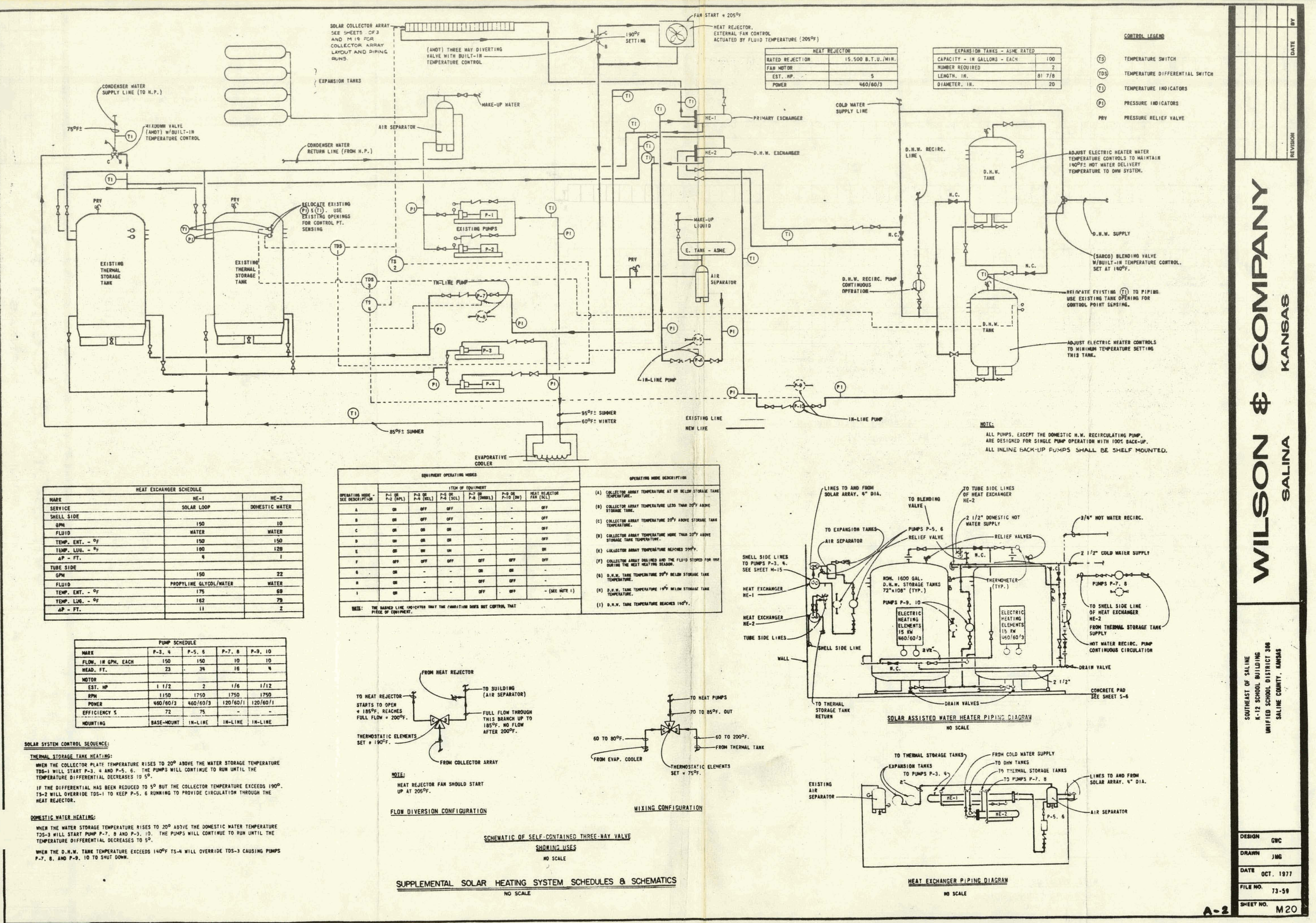




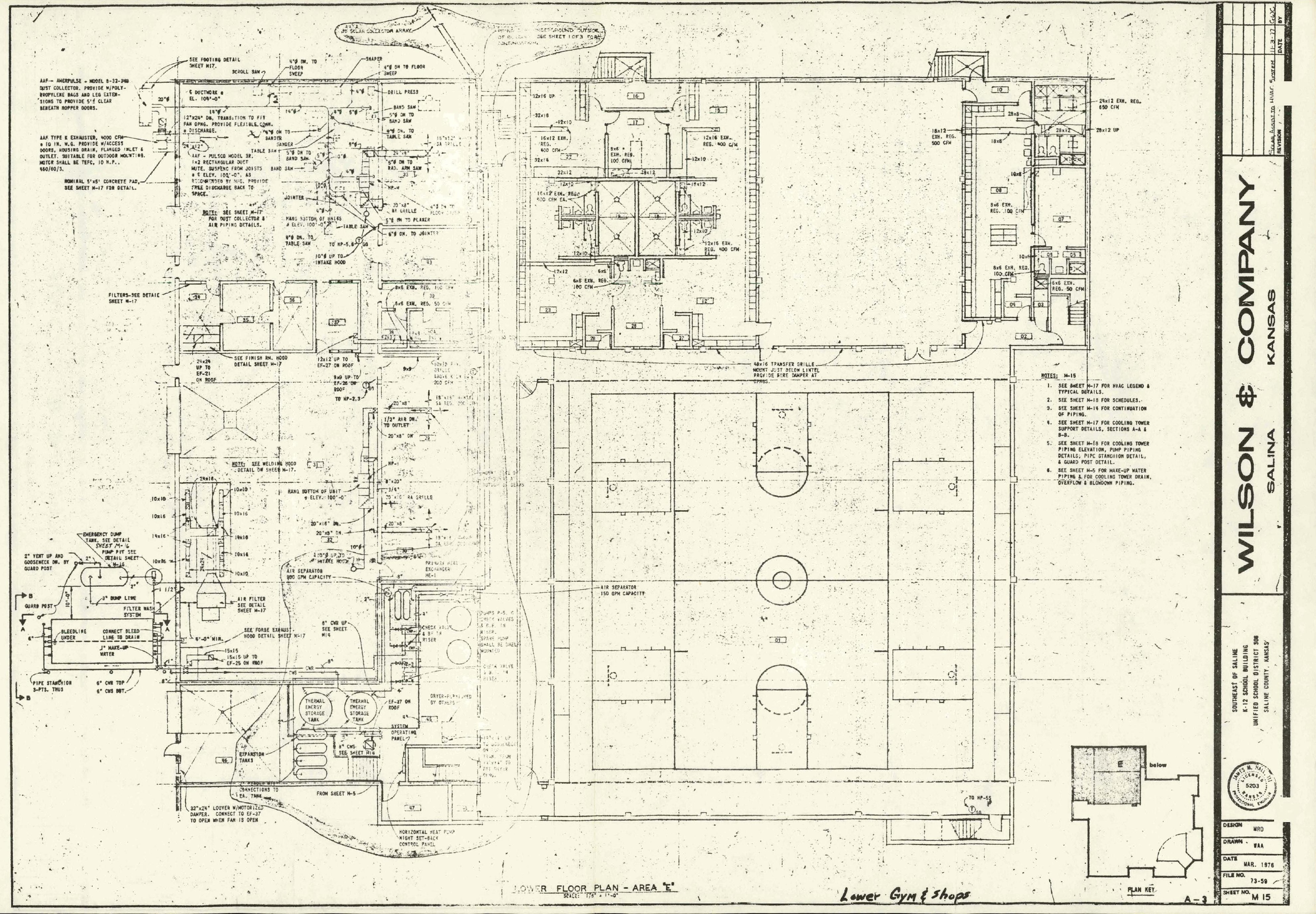




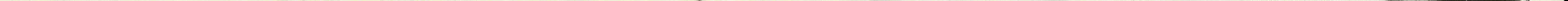




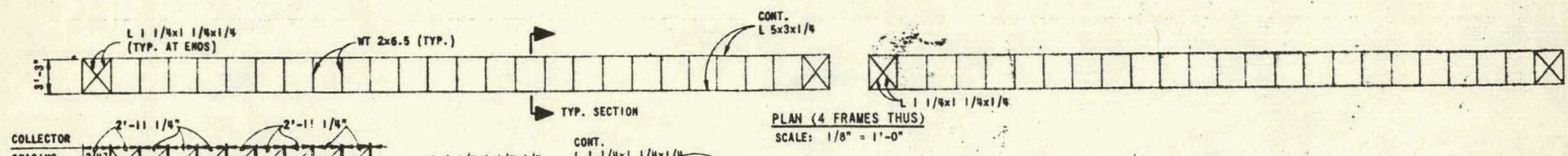
sauta

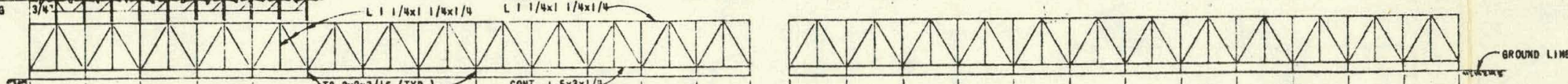

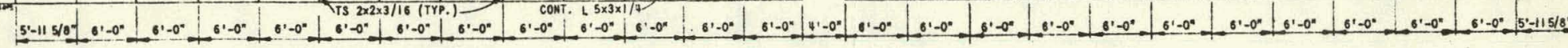

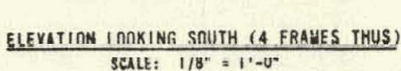

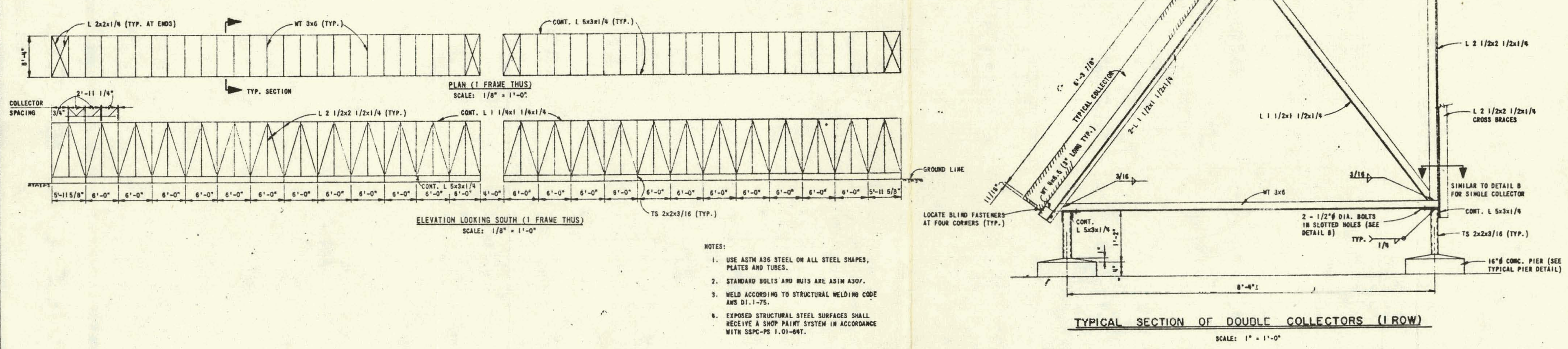

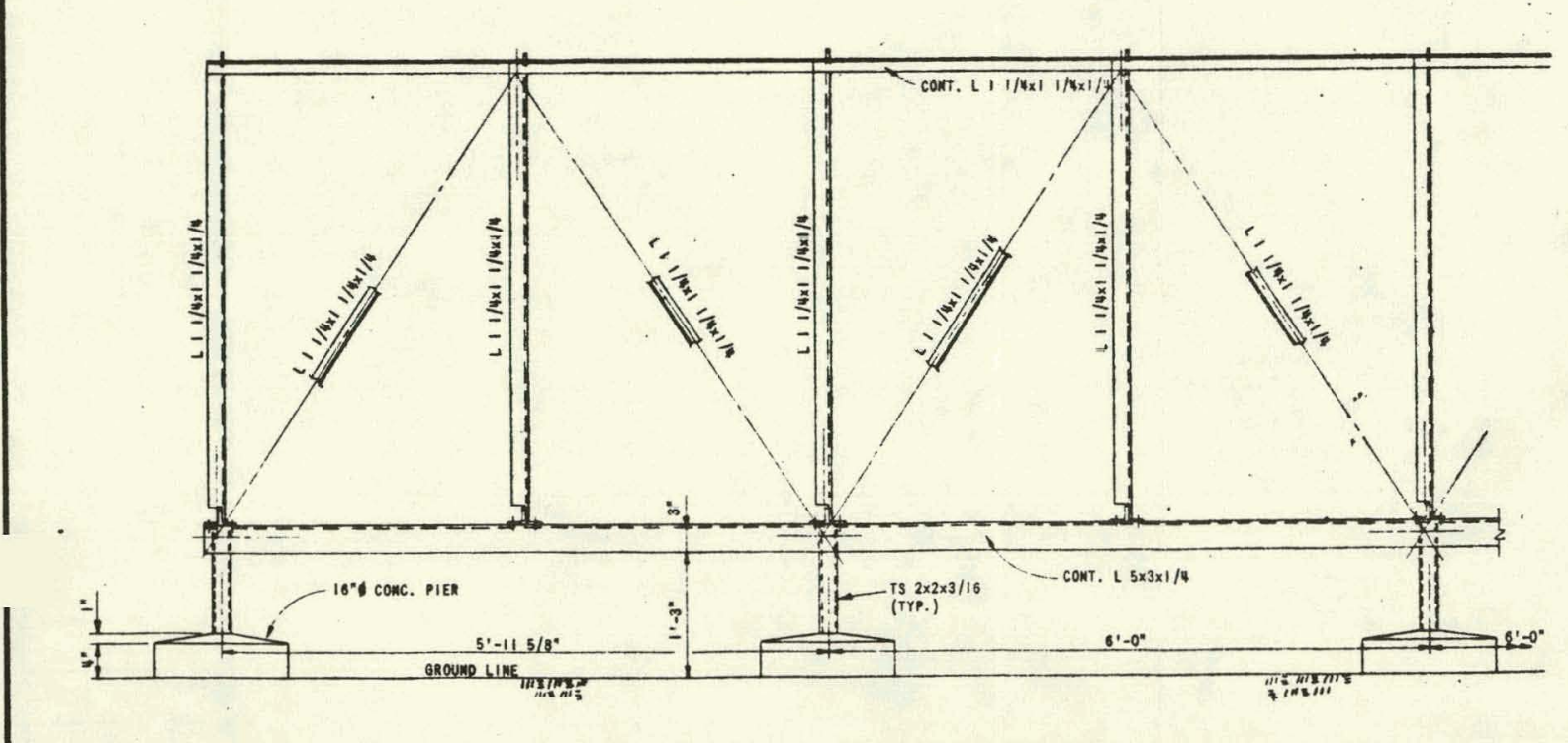

$\frac{\text { TYPICAL ELEVATION LOOKING SOUTH }}{\text { SOME: } 1.211 .00}$
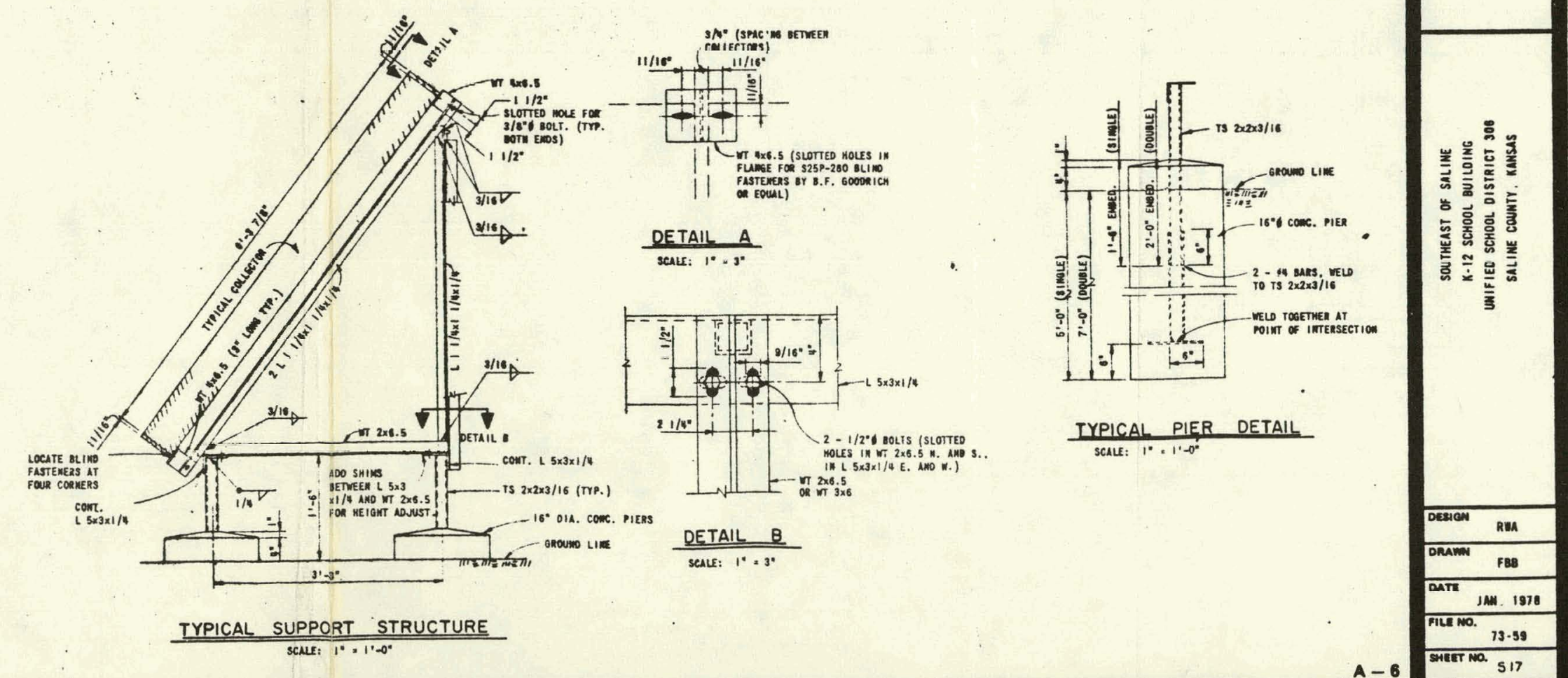


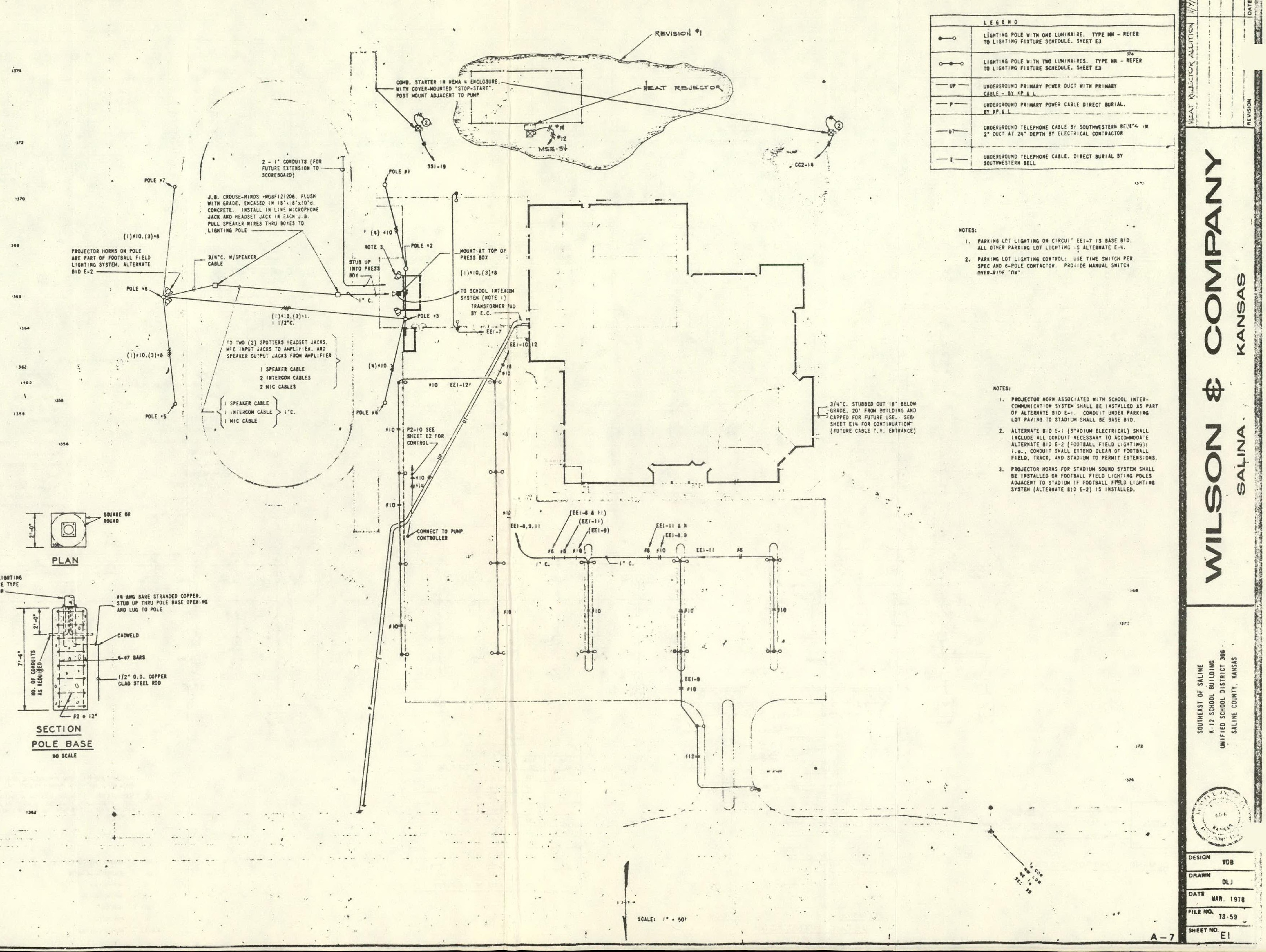




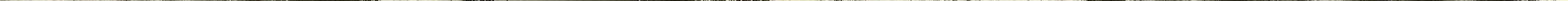




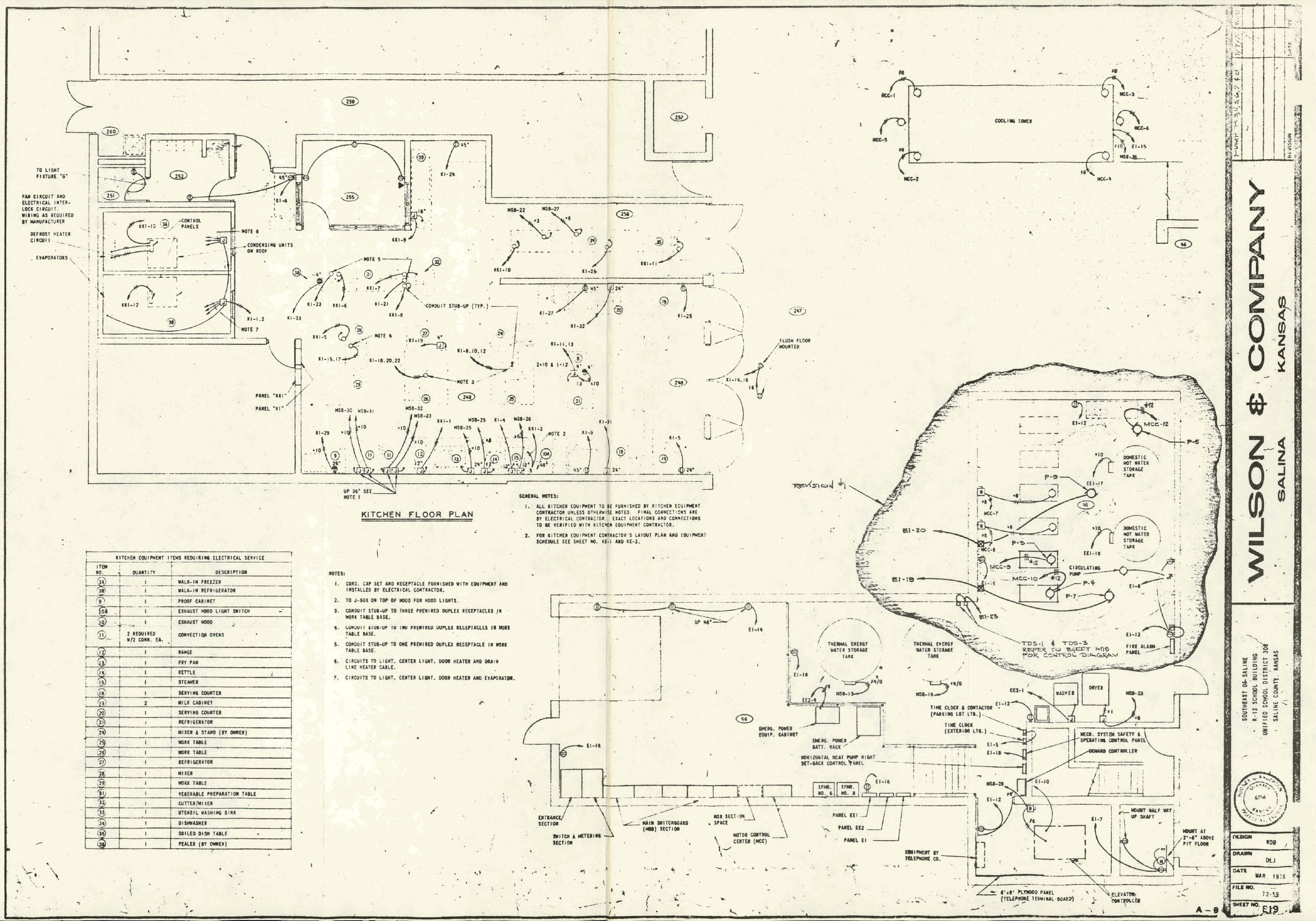




\section{APPENDIX B}

PARTS LISTING AND MANUFACTURERS LITERATURE

PARTS LISTING INCLUDES MANUFACTURERS DATA AND LOCATION ON PLANS.

B1 - 1 SOLAR COLLECTORS

LOCATED SOUTH OF SCHOOL BUILDING, SEE DWG. 1 OF 3 AND M-19

B2 - 1 HEAT REJECTOR

LOCATED SOUTH OF SCHOOL BUILDING, SEE DWG 1 OF 3 AND M-20

B - 3 SOLAR HEAT EXCHANGER

LOCATED IN MECHANICAL EQUIPMENT ROOM, SEE DWG. M-15 AND M-20

B-4 DOMESTIC WATER HEAT EXCHANGER

LOCATED IN MECHANICAL EQUIPMENT ROOM, SEE DWG M-5 AND M-20

B5 - 1 PUMPS AND ACCESSORIES

LOCATED IN MECH. EQUIP. ROOM, SEE DWG M-5, M-15, AND M-20

B6 - 1 AIR SEPARATOR

LOCATED IN MECH. EQUIP. ROOM, SEE DWG M-15 AND M-20

B-7 EXPANSION TANKS

LOCATED IN MECH. EQUIP. ROOM DWG M-15 AND M-20

B8 - 1 RELIEF AND REDUCING VALVES

B9 - 1 THERMOSTATIC VALVE

LOCATED IN MECH. EQUIP. ROOM DWG M-15 AND M-20

B10 - 1 TEMPERATURE CONTROLS

LOCATED IN MECH. EQUIP. ROOM AND ON EQUIP., SEE DWG M-2O

B11 - 1 PRE-INSULATED UNDERGROUND PIPING

LOCATED SOUTH OF SCHOOL BUILDING, SEE OWG 1 OF 3

B12 - 1 PIPE INSULATION

SEE DWG M-5, M-15, M-19 AND M-20

B13 - 1 THERMAL ENERGY STORAGE TANKS

LOCATED IN MECH. EQUIP. ROOM, SEE DWG M-15 AND M-20

B14 - 1 BLIND FASTENERS

LOCATED ON COLLECTORS, SEE DWG S-17 


\section{COST EFFECT DESICN SOLAR COLLETORS}

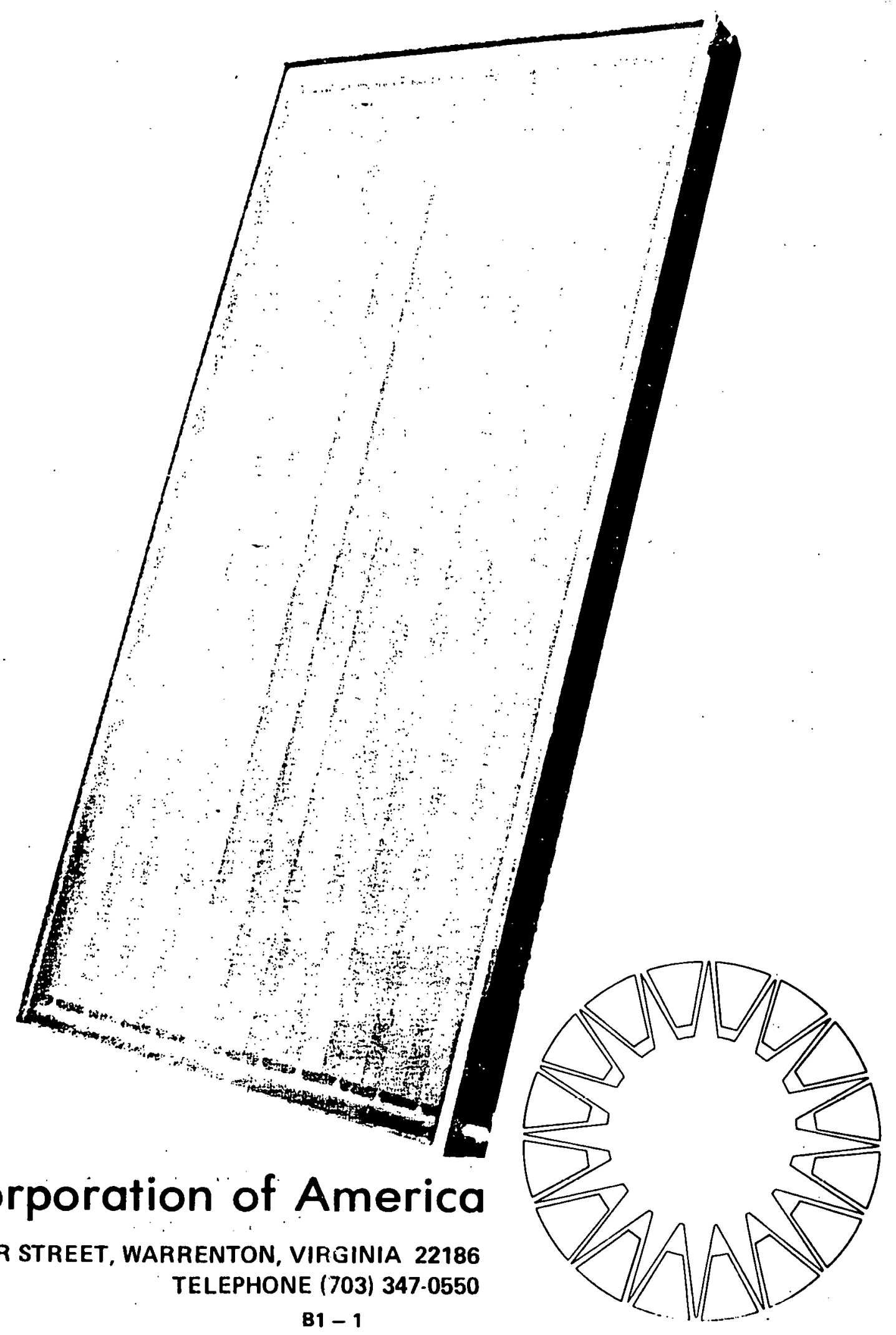

Solar Corporation of America

19 WINCHESTER STREET, WARRENTON, VIRGINIA 22186

$$
\text { B1 - } 1
$$


The CED Collector is Cost Effective Because:

It provides rapid roof top or ground mounting clips to reduce costly field labor. Installation costs can run over $25 \%$ of collector costs.

Large $15 / 8$ " OD Copper fully insulated manifolds are available inside the box to eliminate the need for unsightly exterior piping, which can add $\$ 2.00$ per foot plus rooftop brazing labor and insulation.

Quick connectors are available to rapidly jo in a row of boxes together up to 150 feet long without brazing or soldering. Can be taught easily to unskilled labor.

SCA boxes are $4^{\prime} \times 8^{\prime}$ to fit building standard modules and to require fewer connections on the roof.

SCA boxes are structural, requiring support only at the ends to provide the correct tilt angle.

All SCA CED collector materials are designed to last 25 years or more. Other collectors which use black paint will have to be recoated in 5 years, according to the paint supplier.

SCA has designed for the highest available efficiency so that a small collector area will supply the heat requirement. This saves both collector initial cost and installation cost. In addition, the SCA thermal control design permits double glazing efficiency with a single glaze.

CED provides expansion joints in the manifolds and in the boxes.

CED permits on-the-roof glass replacement should that ever be required.

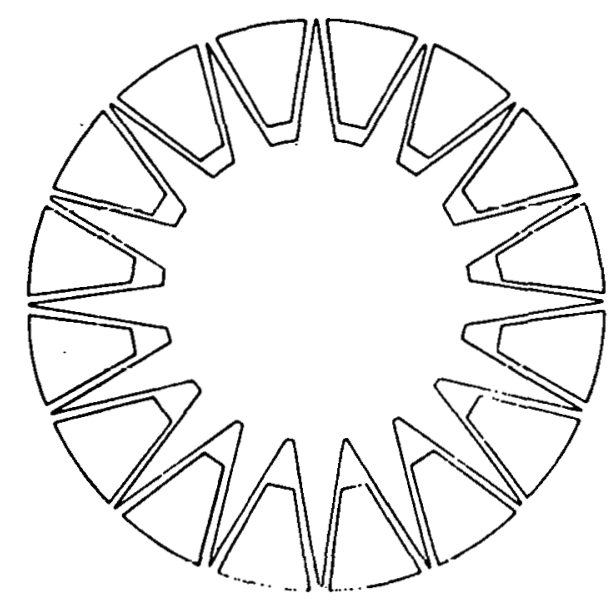

\section{Solar Corporation of America}




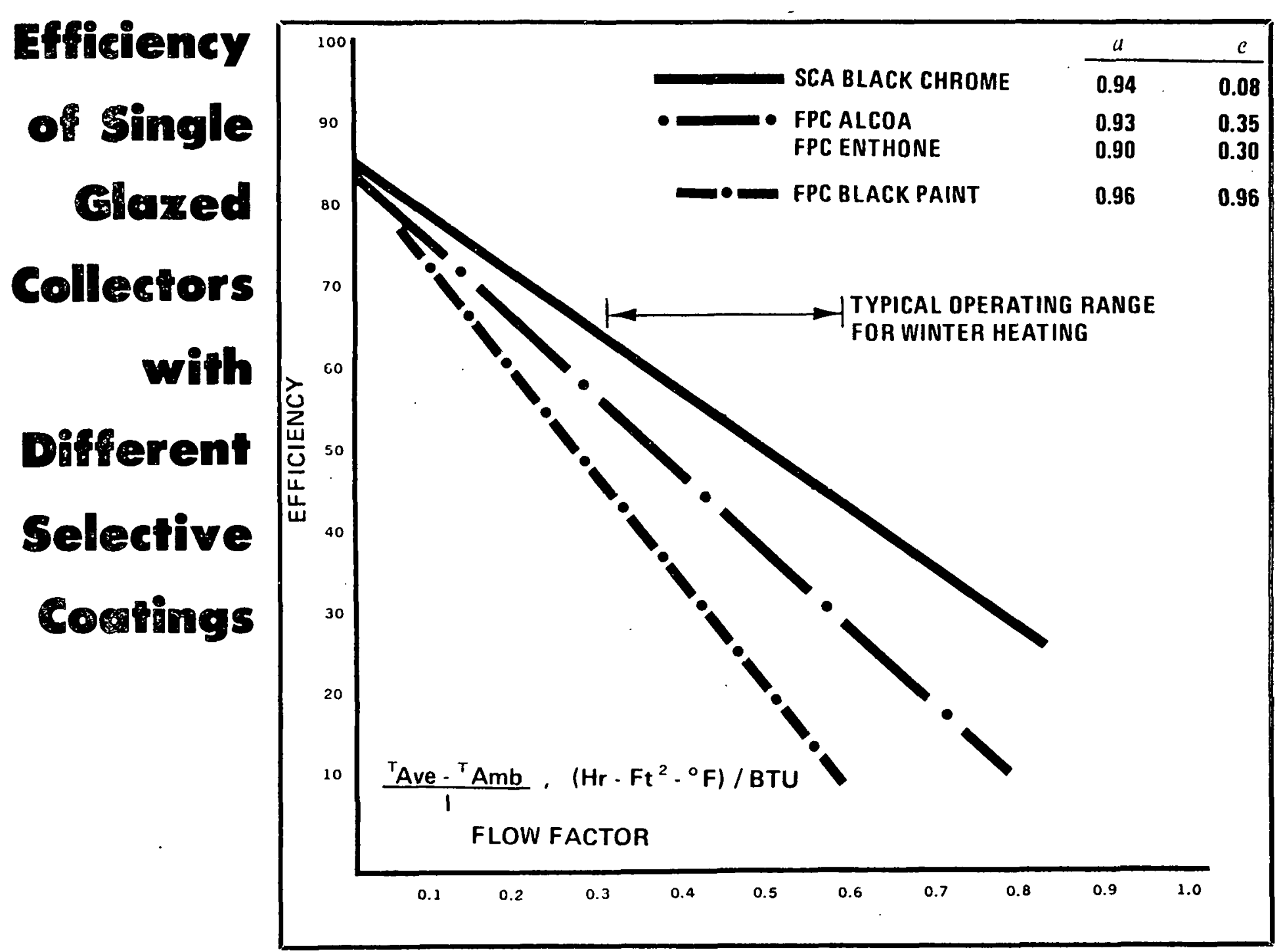

\section{CED Mark IV Solar Collector}

Solar Corporation of America offers a high technology solar collector; designed to last the term of your mortgage and to keep producing the most heat for the installed dollar available with current technology. We believe we are the only company who offers all these top features:

- Custom design service.

- Collector can use water or any conventional coolant.

- All copper liquid system for corrosion resistance.

- Tempered, high transrnittance $3 / 16^{\prime \prime}$ glass in one or two pane styles.

- High temperature seals for dry plate: temperature resistance.
- Black chrome on absorber for highest thermal performance.

- Fiberglass insulation in galvanized steel box modules.

- Uniroof ${ }^{T M}$, Uniside ${ }^{T M}$, concentrating, planar, and freestanding designs.

\section{*Installation} *Service

- For heating and cooling or heating and hot water only.

- System engineered for lowcost installation.

- Installation available; collector only or complete system.

- Two-year limited warranty.

- Extended warranty and maintenance available.

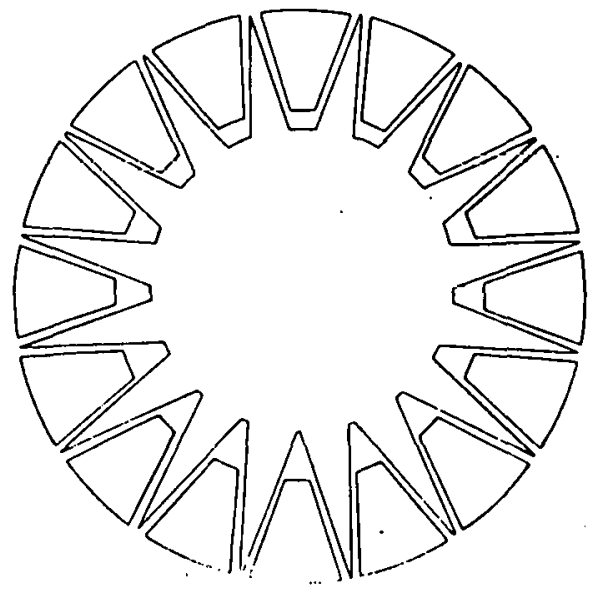




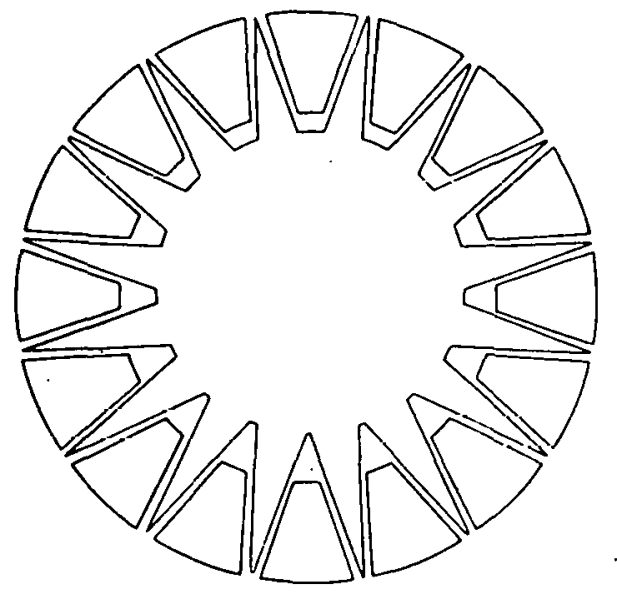

*Design

*Manufacture *Marketing
Solar Corporation of America takes pleasure in announcing its increased line of products:

- Solar Collectors for Buildings and Homes.

- Solar Hot Water Heaters.

- Solar Swimming Pool Heaters.

- Solar Collector Modules for the Do-It-Yourselfer.

- Solar Heaters for Commerce (Restaurants, Motels, Laundries, Car Washes, ....

- Solar Heaters for Industry. (Process Heat)

- Solar Crop Dryers.

Solar Air Conditioning Equipment.
The SCA line of CED (Cost-Effective Design) solar collectors are backed by America's leading solar engineering consultants, InterTechnology Corporation (ITC).

The CED collectors are winning wide acceptance for their life-cycle low cost.

SCA will be most responsive to the needs of both large and small solar requirements.

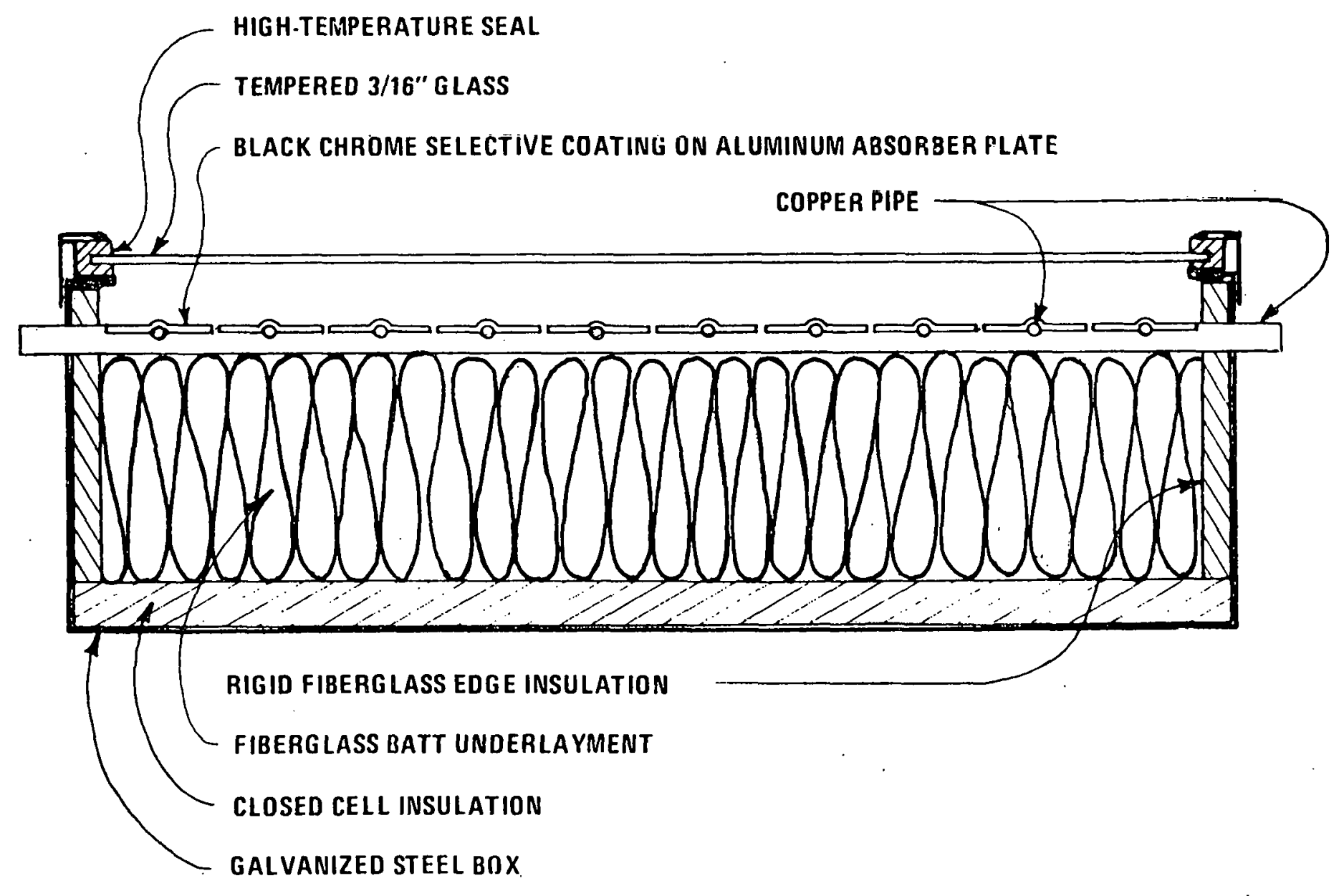




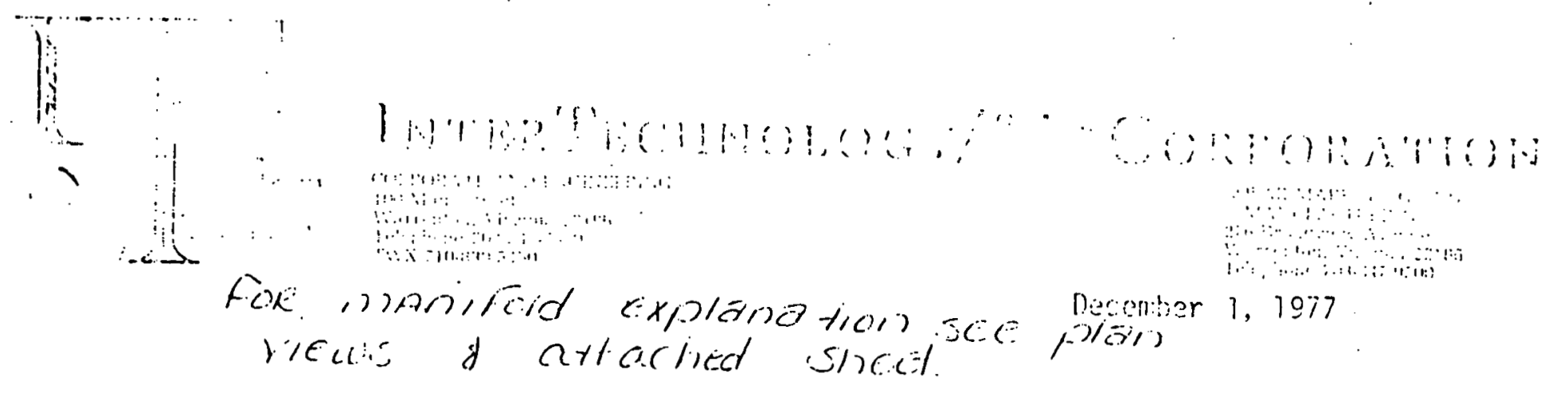

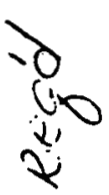

\section{Specification 770 s:01 \\ Solar collector - Hediun Temperature}

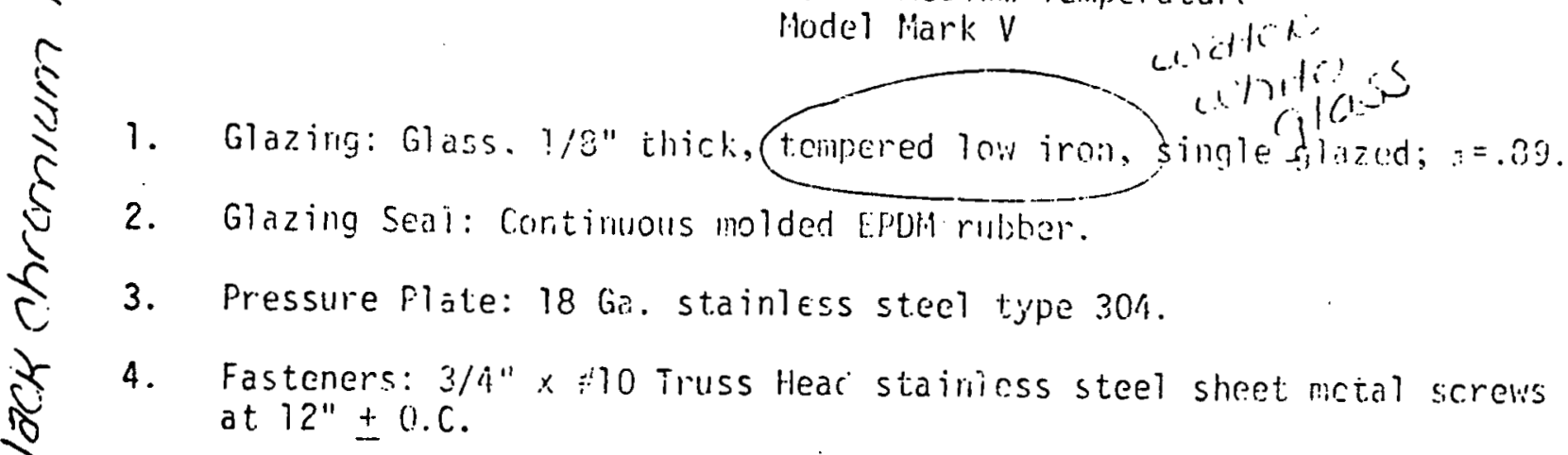

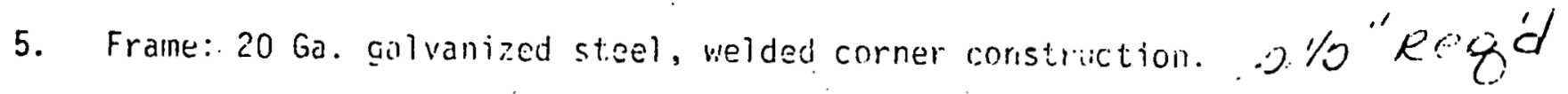

6. Fin Tube Absorber Assembiy.
A. Manifolds: Tro, Marddran, type li copper tubes, (1"joninai.

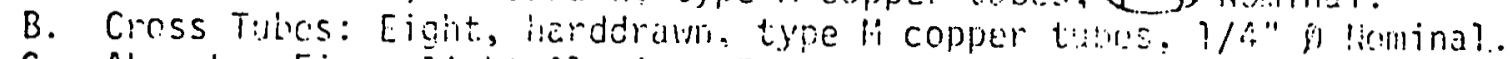

C. Absorber Fins: Eglt Altan Extrusions; . $660^{\prime \prime} \times 22 \times 68.5^{\prime \prime}$.

D. Absorber Coatino E Elack paint $a=0.95 \quad c=0.3$.

7. Manifold Connectors- High temperature formed ribber hose with hose clamps. Silicin?

ix: $\quad$ Insulation - $1 "$ fiberglass around ejges $3 "$ " riberglass under absorber.

9. Working pressure - 50 PSIG; naximium 100 PSIG.

10. Area - 19.0 square feet $\left(353 / 8^{\prime \prime} \| \times 773 / 8 " L \times 51 / 4 " D\right.$ overal) dimensions).

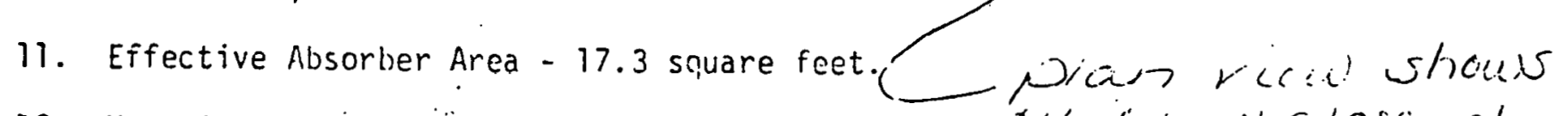
12. Hounting Provisions - Consult factory.

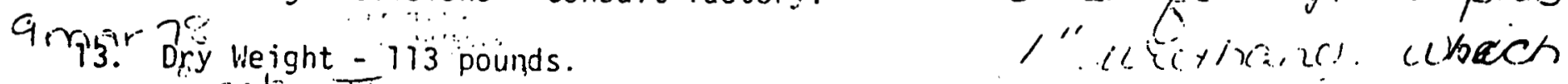

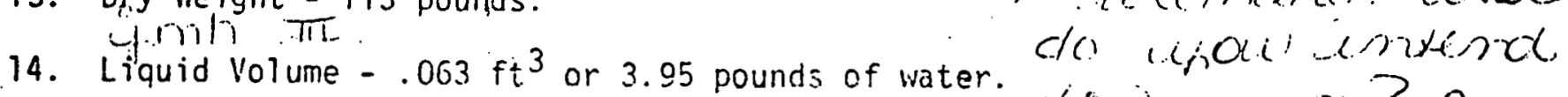

Yas.s

Thiniteld

cibsazber lontiriy
insulation

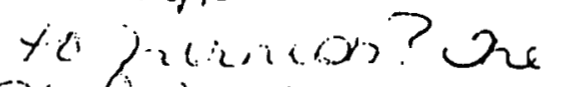

$$
\begin{aligned}
& \text {-pen(m) ins corract. }
\end{aligned}
$$

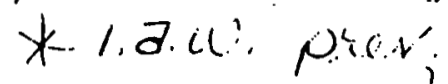

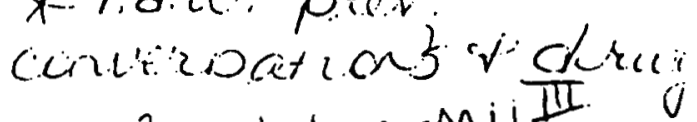

$$
\begin{aligned}
& \text { - Plearanatif JMH Jil U }
\end{aligned}
$$




\section{Retail Pricing and Options Available for}

Model Mark V

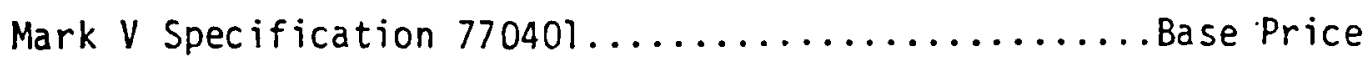

$\$ 208.00$

\section{Options}

1. Water White Glass: High transmittance $\tau=.91 \ldots \ldots \ldots \ldots \ldots \ldots . \$ 11.00$

2. Black Chromium Absorber Coating with $\alpha=0.94 \varepsilon=0.1 \ldots \ldots \ldots \ldots \quad \$ 45.00$

3. Optional manifold 2" or $21 / 2 " \emptyset$ Nominal - Consult factory for pricing.

All option prices are in addition to Mark $V$ base price. Options available in any combination.

Prices effective 7 January 1978. All prices subject to change without notice. All prices exclusive of packing and freight.

Collectors and accessories FOB Warrenton, Va.

\section{Retail Discount Schedule}

Volume (Units)
$\begin{aligned} 1-10 \\ 11-50 \\ 51-100\end{aligned}$
Greater than 100

Discount

$$
\begin{aligned}
& -0- \\
& 5.0 \% \\
& 10.0 \% \\
& \text { Factory Pricing }
\end{aligned}
$$

\section{Order Terms}

$1 / 3$ with order, COD. Net 30 days with approved credit. Orders honored as placed. 
InterTechnology/Mini Corporation

(:URIOURATE: AND ENGINEERING

S(H.AK MIARKFIINL; $A N I)$

MANUFACTIURINC

276 Rrnadview Airnur

Trelephnne 703-347-7900

$\operatorname{Tix} 710-839 \cdot 5450$

IVirrenton. Virminia 2218

Telephrone 703.347 .9500

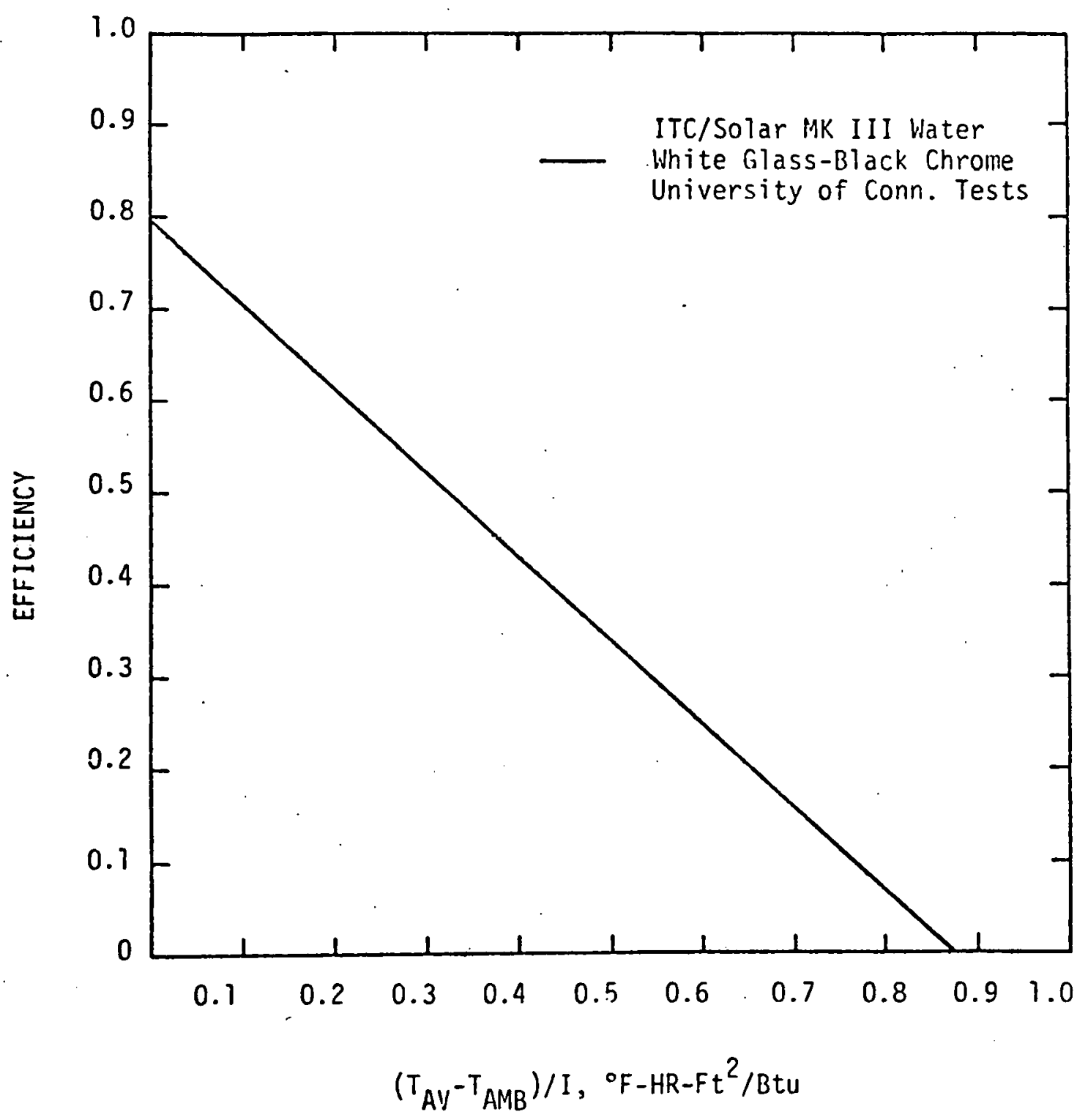

I 


\author{
LIMITED WARRANTY \\ (HUD Solar Hot Water Initiative) \\ InterTechnology/Solar Corporation \\ ("ITC/Solar") \\ 100 Ma in Street \\ Warrenton, Virginia 22186 \\ 703-347-9500
}

PRODUCT

Joule Box ${ }^{T M}$ (Solar Water Heating) System

EXTENT AND DURATION OF. WARRANTY

I. System

A. Extent

Any system failure caused by a defect in either materials, manufacture or installation by ITC/Solar or its distributor or agent will be remedied. All necessary (as solely determined by ITC/Solar) replacement parts, labor, shipping or handling will be provided without charge to the owner. Where installation is performed by the owner, the warranty covers only defects in materials or manufacture and is limited to the provision, at the site and without charge to the owner of a:11 parts necessary (as solely determined by ITC/Solar) to remedy the defect.

B. Duration

This warranty is effective for one (1) year from the date of completion of the initial installation. Any warranty replaced item will carry only the unexpired portion of the original warranty.

II. Solar Collectors

A. Extent

1. Defects in materials or manufacture

All necessary (as solely determined by ITC/Solar) replacement parts, labor, shipping or handling will be provided without charge to the owner.

2. Corrosion of the absorber plate and other coolant passages which adversely affects performance of the system

All necessary (as solely determined by ITC/Solar) replacement parts, labor, shipping or handling will be provided without charge to the owner. (NOTE limitation in B below.)

B. Duration and special limitation

Excluding corrosion defects described below, this warranty is effective for five (5) years from the date of completion of the initial installation. HOWEVER, the warranty for corrosion defects arising more than one (1) year from the date of completion of the initial installation covers only the cost of any parts or new collectors, delivered to 
the site, necessary (as solely determined by ITC/Solar) to remedy the defect. Any warranty replaced item will carry only the unexpired portion of the original warranty.

\section{Other Components}

The other components (tanks, pumps and controller) used in the Joule Box TM System carry their own manufacturer's limited warranty. ITC/Solar and/or its distributors pass these warranties to the owner at the point of sale. In particular, ITC/Solar warrants against defects in materials and manufacture of heat exchangers and tanks for a period of five (5) years. This warranty shall include shipping and handling but not include removal and reinstallation.

\section{OWNER RESPONSIBILITIES}

If the joule Box ${ }^{\text {TM }}$ System is installed by parties other than ITC/Solar or its distributor or agent, the owner is responsible for insuring that the installation is made in accordance with ITC/Solar specifications.

A) though ITC/Solar and/or its distributor will promptly inspect the system.at the site to verify failure, establish probable cause, and determine corrective action, if the inspection reveals no warranty related defect, a reasonable charge may be made to the owner for the inspection. Any item to be replaced must be made available for inspection in exchange for a replacement.

HOW TO MAKE A CLAIM

A claim under this warranty should be made to the distributor from whom the system was purchased. If this cannot be done, ITC/Solar should be contacted directly.

\section{ITEMS NOT COVERED BY THIS WARRANTY}

ITC/Solar and/or its distributors are not responsible for any damage to the glass or other components of the system arising from causes not attributable to defects in materials, manufacture or installation. Damage attributable to natural disaster or other acts of God is specifically excluded from coverage under this warranty.

ITC/Solar and/or its distributors are not liable for any incidental or consequential damages or any payment other than for repair, replacement or refund of the purchase price paid for the system. NOTE: Some states do not allow the exclusion or limitation of incidental or consequential damages. Therefore, this limitation or exclusion may not apply to the owner.

\section{GENERAL}

All warranties shall continue, to the extent of the warranty period remaining. to any subsequent owner of the system, provided that the new owner contacts ITC/Solar or the distributor within thirty (30) days of purchase to establish 
the new ownership, have questions answered and obtain the system operating and mainterlance manuals.

In the absence of other suitable proof of the date of completion of the installation, the effective date of this warranty shall be based upon the warranty registration records maintained by $I T C /$ Solar or its distributor.

THIS WARRANTY GIVES THE OWNER SPECIFIC LEGAL RIGHTS. THE OWNER MAY ALSO HAVE OTḦER RIGHTS WHICH VARY FROM STATE TO STATE.

Warranty Registration

Promptly Return to Seller

liame of Owner

Address of Owner

Model Number

Date Purchased
Name of Dealer

Address of Dealer

Serial Number

Date Installed 

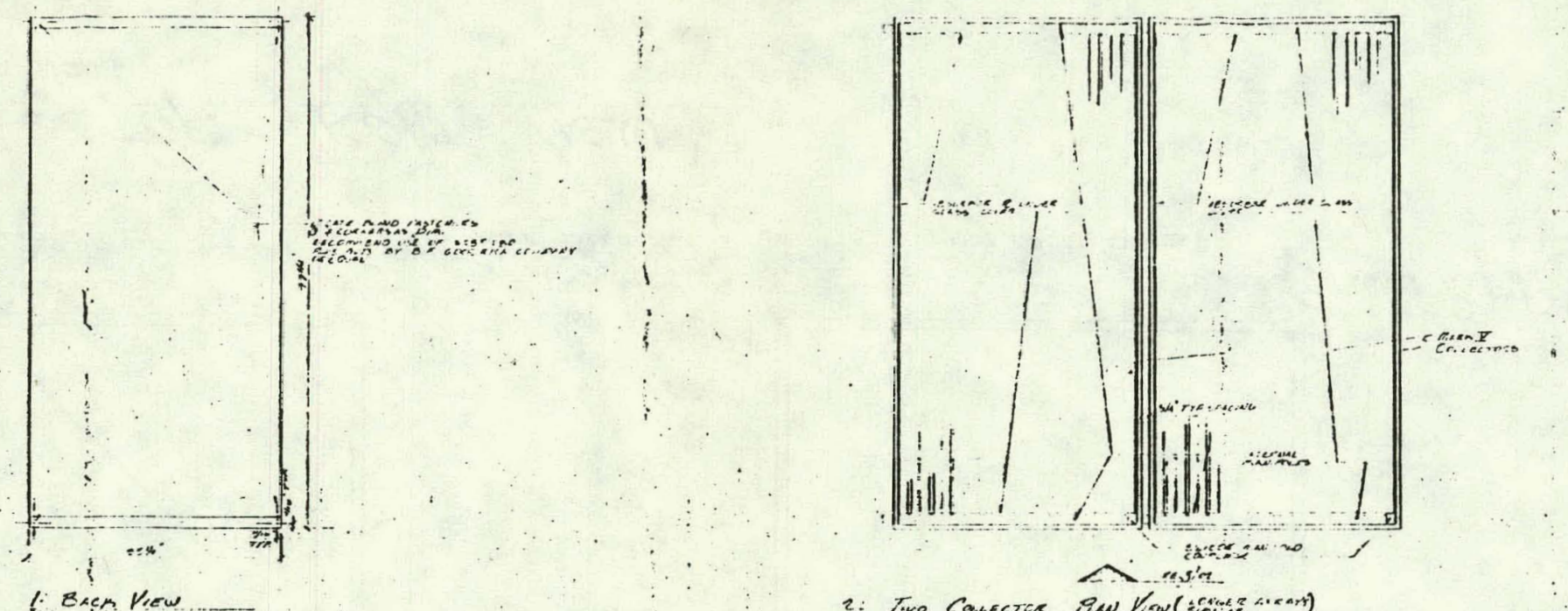

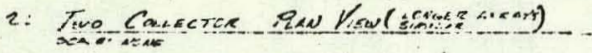

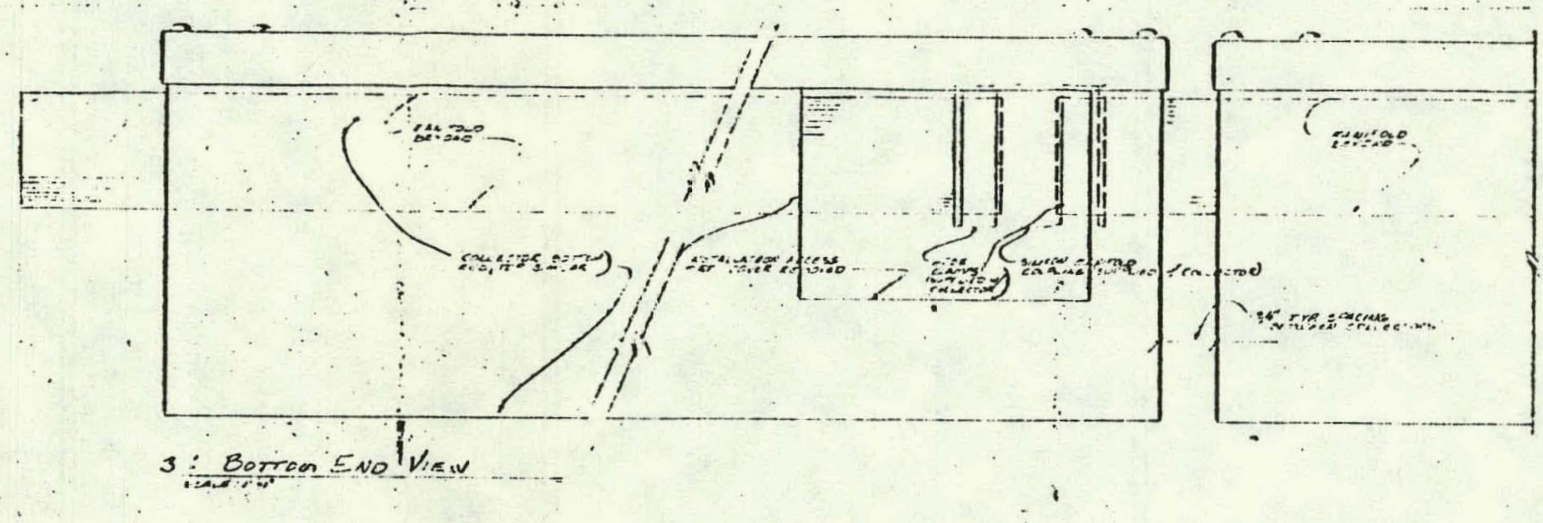




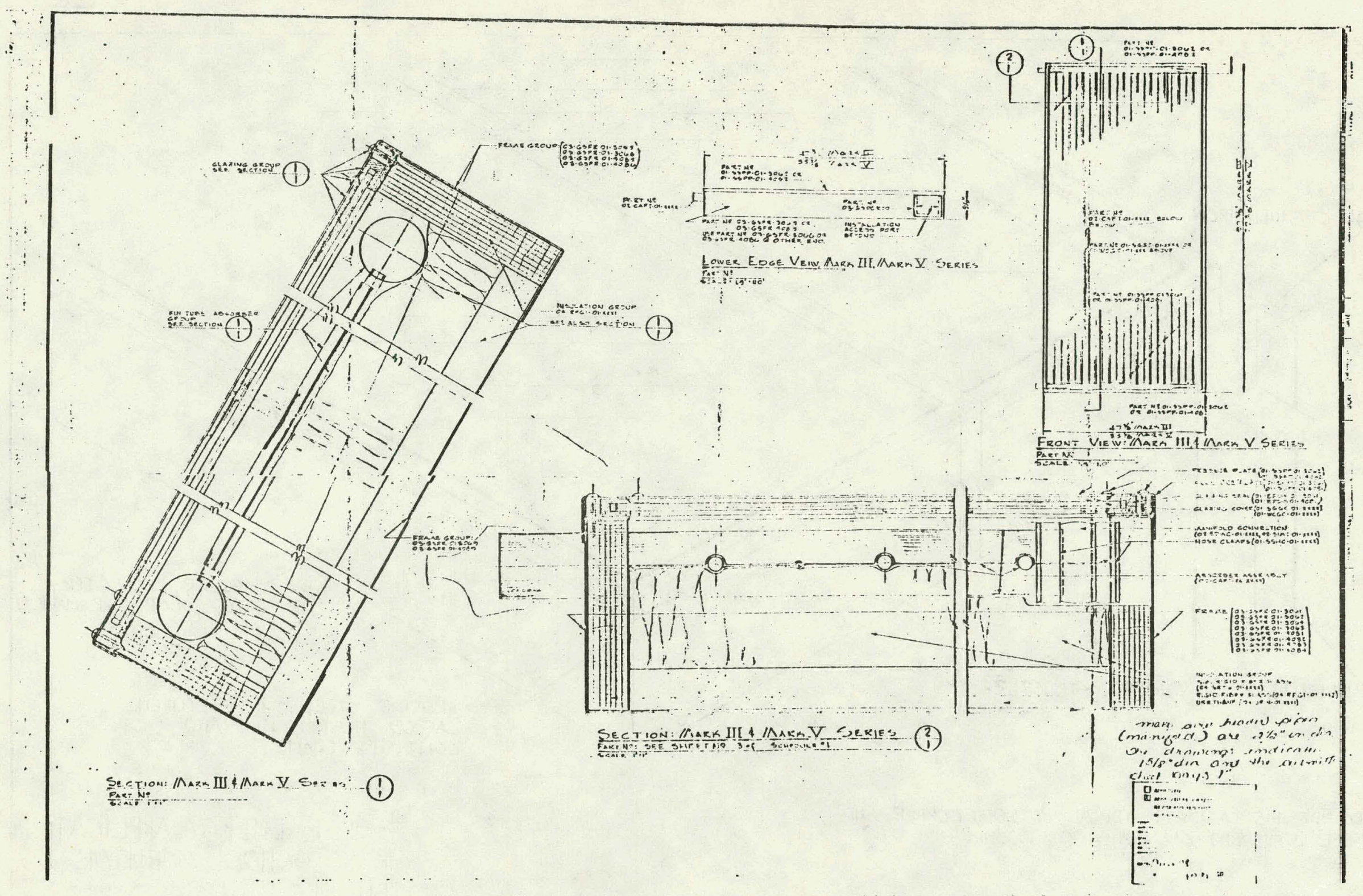




\section{RECELIVIik:}

Insuectiun

- Inspect unit ior shipping diunde (espoially core) before uncrating.

- Sotate fan which should nove freely. Note that one blade is wired to guard on sone models for shipping. Hemove wire before rotating ran.

Hundling

- Excercise care to avoid damare to cort.

- All units are shipged with wool skid facilitating rork lift handing. Lifting holts are provided in MWC and sune HC side nembers. HC units have integril us renovable lifting lup.s (shipped loose on'some inodels).

\section{Storage}

- Units normally have one coat of medium gray, semi-tiloss (chlorinated rubber base) enumel paint. A finjsh coat of paint should be applied to all exterior lerrous suriaces unless storage is indoors and no significant corrosion started during shipment.

- Do not paint core as this may impair air tolow and heat transfer.

- All openings are sealed with appropriate pipe plugs, pipe caps, thread protectors, liunse suvers and/or pressure caps at the lactory. Be sure these are in plice.

\section{INSTALLATION}

Location

- Insure that no obstructions impede either discharge or inlet air flow. If unit is mounted in wall, provide auple room ventilation.

- MWC units should be oriented to take advantage of prevailing winds to avoid recirculation and the resultant loss of cooling effect. Expected wind direstion on hot, summer days should especially be anticipated.

- Duct work on housine of unit adjs air flow static resistance which must be overcone with extra ian capacity. Ducting should be larger in area than heat exchariger cure iace and contain only straight runs ani long radius turns.

Pound:ttion and lounting

- Flexible connections or non-rigid plubing (lans:ars und right-angle bends) should be used to allow thermil expansion of core, inculation from vibration and freedom from static loading.

- Consult "cooled equipment" manufactirer tor recommendations on specific plunbing systern. Inlat to heat exchanger is nornslly at the highest cormection, but this is not essential.

- In the aa.je of round tuts corcs with counter-1low pastes, plumb inlet fluid to annection farthest from blower fan to avoid co-ilow of thie fluid and cooling air.

Pan Drive Sisteri

- If lan i.; motur jriven, check thit voltaie, irequelisy and number of mhases un motor nunf:plate are compatible with electrical supply.

- lonnect iastor leads so that rutiltion oi forced dript blower ian is counler-cliwise when viewed frow tan side or unil unless fan is supplied with special, lelt-hand blades.

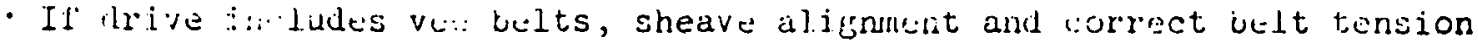

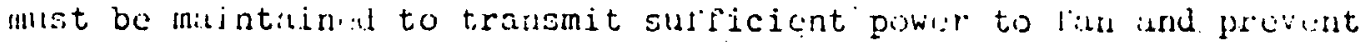
écossivis belt and buaring wear.

- If lan is entive driven, the pafterred lucation for the idler sheave is

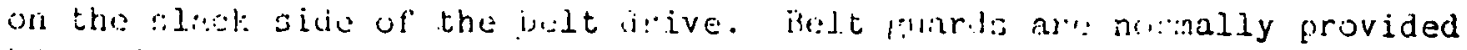

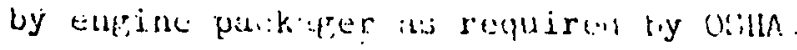




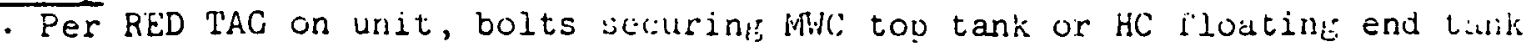
to side members must be loosentid to permit differential themal expansiun of steel sides and nonferrous : jru.

- It tube fluid is niter, liil unit lrom a cleun and chenicil rree source. Use a Jü aquious etijlene rlycol solution if freezing is possible. For ambient conciticas telow -25F(-3ii) use a $60 \%$ sclution, ielow $-50 F(-45 \mathrm{C})$ consult isactory.

- Check unit for undue vibration resulting irom ian imbalance or loose dirive components.

\section{MAIINENANCE}

Motor

- iall vearings should be lubricuted once per year or each nine months for sever duty moturs, li hp or lareer. Consult locil motor manuacturer representative ior broase type specificution.

- Clean ventilaticri openines as needed.

Fan Bearings (Vee Eelt \& ixternil Drive Systems)

- Fun anu idler bearings are provided with conoination Alemite grease and reliel titings and should be lubricaled once per year or more often for scvere duty appliciations.

- Eearinf tempiriture shoula ne:sr exceed 150-160F.

Fan Drive

- Check and mintain allgnmsrit of shesves by adjustine location cn shaft and adjusting ijlluw bloci base.

- Check and maintain cyerect tension of fan belts by adjusting iler.

- Check ior frate vibrijicn jus to iar. imoalance, cearing wear ijo loose drive colipunents.

\section{Core ind Frume}

- Periodically ciack for core or pluming lestigge.

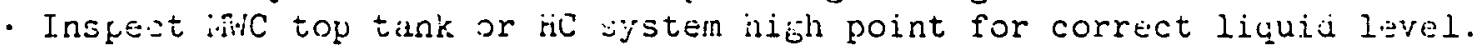

- Test anti-fresze coolant for correct specific gravity before cold weather.

- Exanize interior of lwil ton tink througn riller neck for sizns of scale

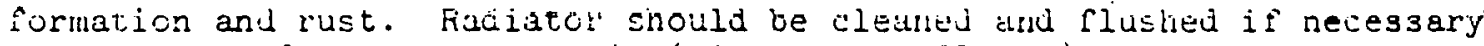

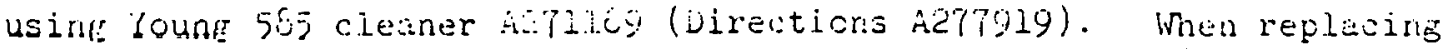

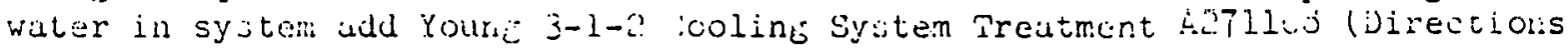
A<7\%y:o)

- If eteel irane jevelops rus: cloan and repaint as necessary.

- Dirt acumulation on : ore ä: ve removeu by orushing and blswing with

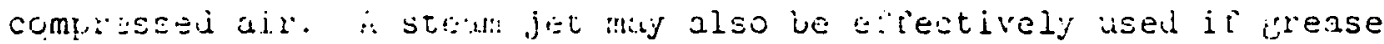
defosits are ch core. liuts: re curefui tilat fins o: tubes are not

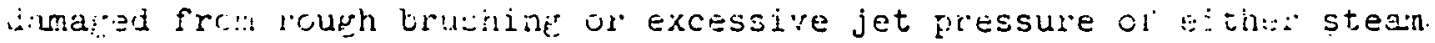
or $: i i r$.

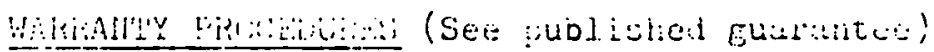

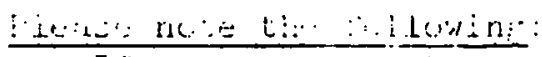

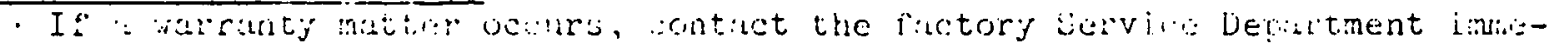

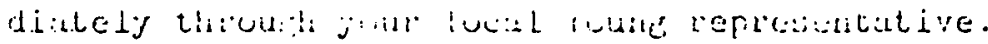

- In all communi:etions wita jervice Department, include complete nanerlate idita.

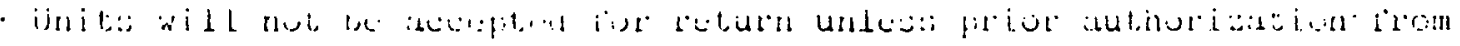

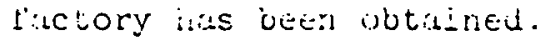

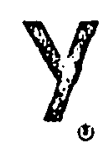

Torn: :Tic $;-5-i$

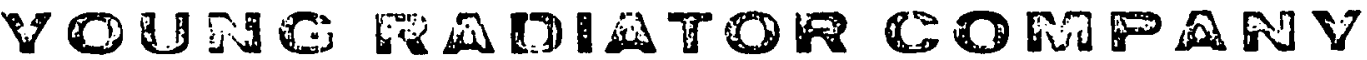

2825 Four Mile: Road o Facine. Wisconsin 5.3404

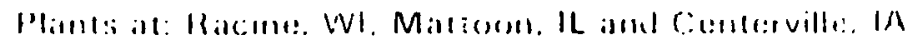

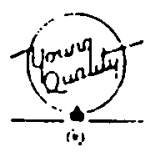

n:27\% 


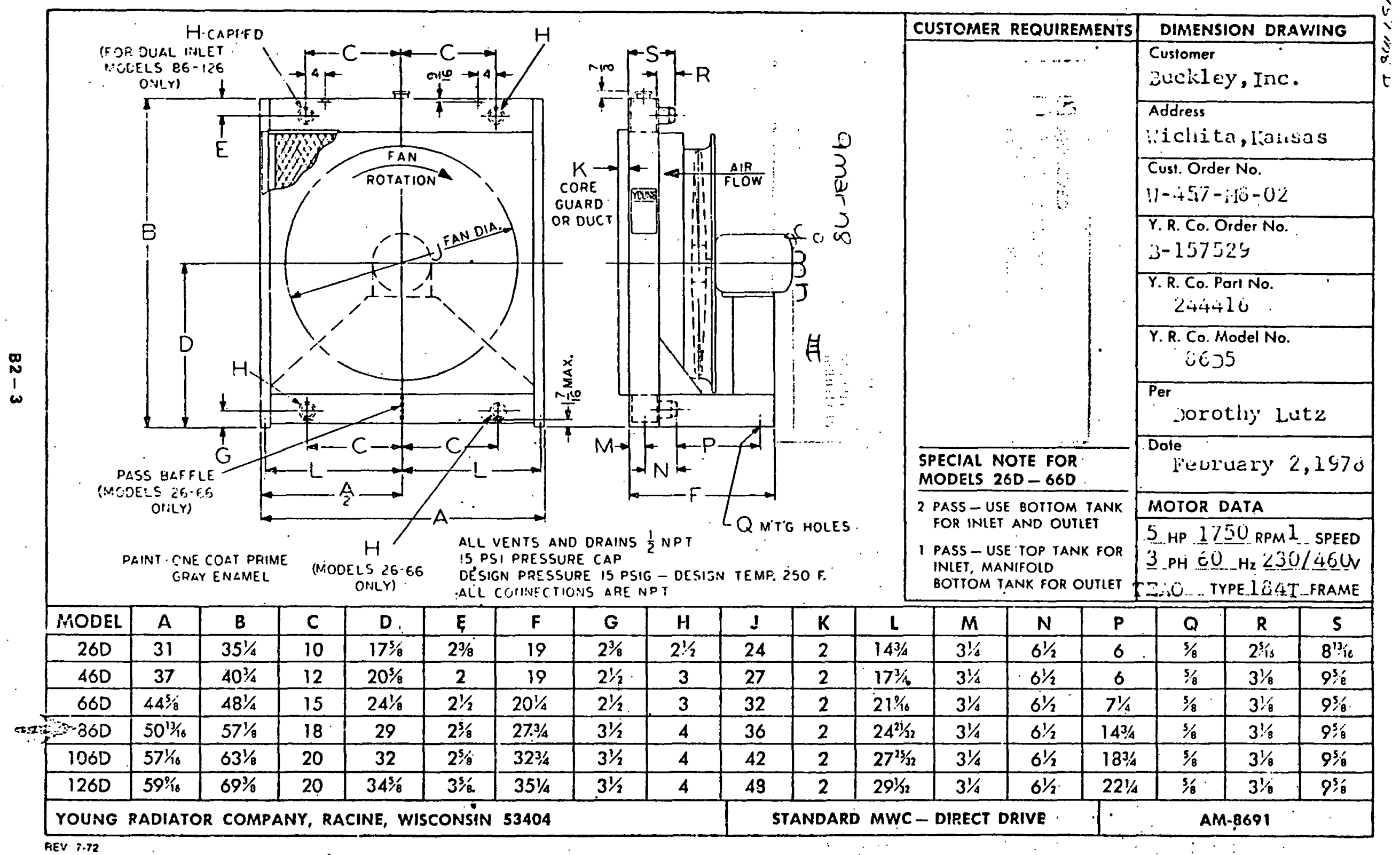


This page has been removed because of copy restriction. For information on the solar heating $150 \mathrm{gpm}$ heat exchanger, unit number 4CT-14-90, contact Richmond Engineering Company, Richmond, Virginia. 
This page has been removed because of copy restriction. For information on the domestic hot water heat exchanger, unit number 2CT-05-36, contact Richmond Engineering Company, Richmond, Virginia. 


$$
\begin{aligned}
& \text { Buckley, Inc. sec difiched } \\
& \text { w-457-M8-05 sheet for explanaticins } \\
& \text { 2-9-78 } \\
& \text { J-9432 }
\end{aligned}
$$

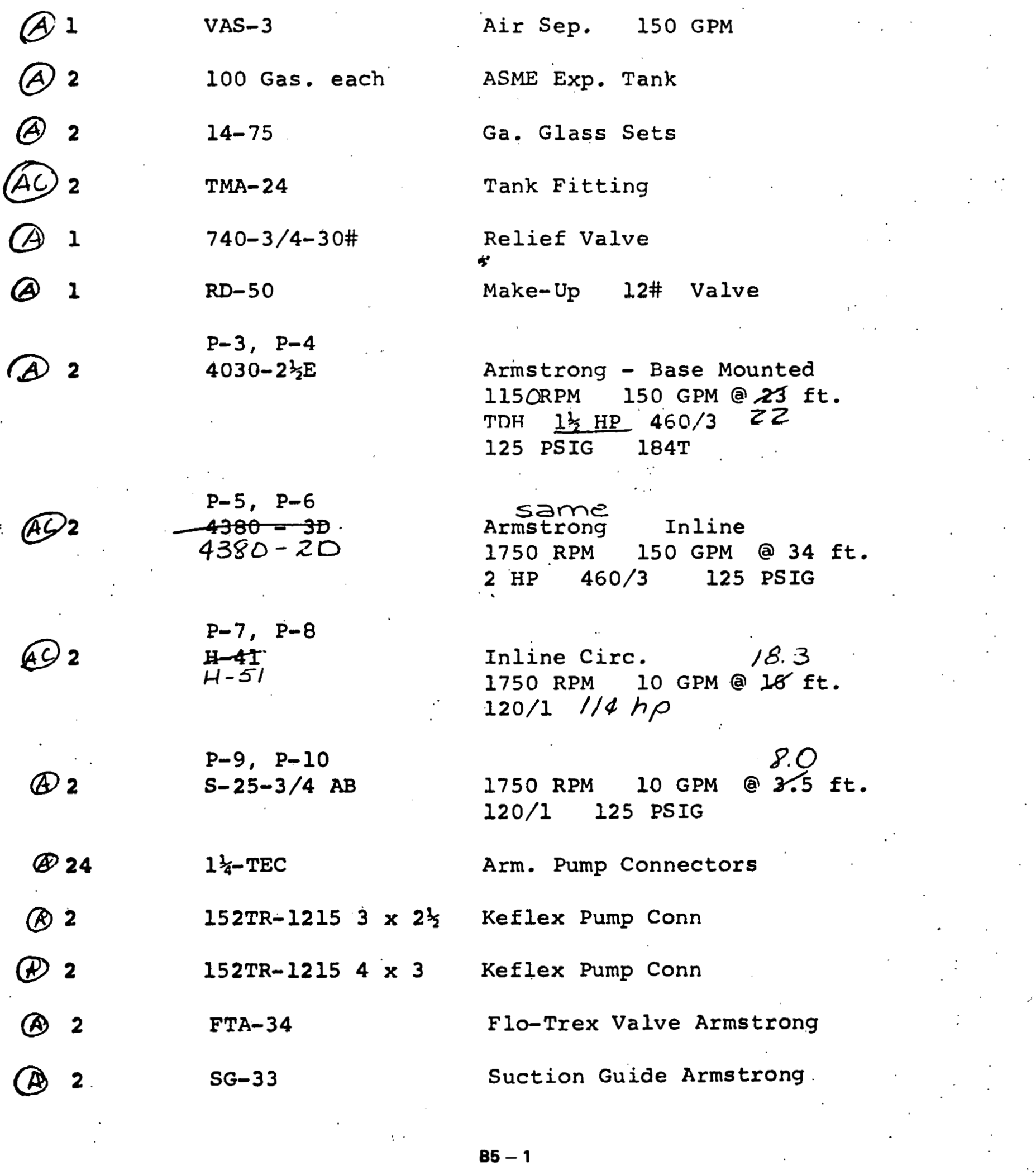


now $g w C$

WILSON

COMPANY

architects? date: uso \#306 fol le $73-59$

$\because \because: \because: 3$ saner shop drawings

$p-5,6$

due to the pumps having to handle wide variations in total dynamic head i believe the 4320-20 would be the best choice

$p=-7,8$

your submittal data, other than the cover sheet, shows the $1-51$ ass the selection while the cover sheet shows the $\mathrm{H}-4 \mathrm{l}$ due to the submittal heat exchangers having a higher pressure drop than expected $i$ think the $H$ - 51 to be the pump needed.

BS - 2 


\section{ARMSTRONG CENTRIFUGAL PUMPS SUBMITTAL DATA}

\begin{tabular}{|c|c|}
\hline $\begin{array}{l}\text { FILE NO } \\
\text { DATE JON }\end{array}$ & $\begin{array}{l}.96 \\
1968\end{array}$ \\
\hline $\begin{array}{l}\text { SUPERSEDES } \\
\text { DATE }\end{array}$ & NF: \\
\hline
\end{tabular}

\section{SERIES 4030 TYPE MB}

\begin{tabular}{|c|c|c|c|}
\hline \multicolumn{4}{|c|}{ CUSTOMER } \\
\hline \multicolumn{4}{|l|}{$\bar{x}$} \\
\hline \multicolumn{4}{|c|}{ ENGINEER } \\
\hline \multicolumn{4}{|l|}{. } \\
\hline \\
\hline \multicolumn{4}{|c|}{ कीTIFT" SAECIFICATION REFERENCE } \\
\hline \multicolumn{4}{|c|}{$\because \ldots \ldots$ ank $\ldots \ldots$} \\
\hline \multicolumn{4}{|c|}{ PUMP DESIGN DATA } \\
\hline \multicolumn{2}{|l|}{$\begin{array}{l}\text { PUMP } \\
\text { SIZE }\end{array}$} & \multicolumn{2}{|l|}{ LIOUID } \\
\hline \multicolumn{2}{|c|}{$\begin{array}{l}\text { CAPACITY } \\
\text { U.S.G.P.M }\end{array}$} & \multicolumn{2}{|l|}{ TEMP. } \\
\hline \multicolumn{2}{|l|}{$\begin{array}{ll}\text { FEET } & 23 \\
\text { HEAD } & 23\end{array}$} & \multicolumn{2}{|l|}{$\begin{array}{l}\text { SPECIFIC } \\
\text { GRAVITY }\end{array}$} \\
\hline \multirow{2}{*}{$\begin{array}{l}\text { PART } \\
\text { NAME }\end{array}$} & \multicolumn{3}{|c|}{ CONSTRUCTION } \\
\hline & $\begin{array}{l}\text { ALL. } \\
\text { IRON }\end{array}$ & $\begin{array}{l}\text { BRONZE - } \\
\text { FITTED }\end{array}$ & $\begin{array}{ll}\text { ALL- } \\
\text { BRONZE }\end{array}$ \\
\hline $\begin{array}{l}\text { VOLUTE } \\
\text { I MPELLEA } \\
\text { COVERPLATE }\end{array}$ & $\begin{array}{ll}\text { CAST IRON } \\
\text { CAST IRON } \\
\text { CAST IRON }\end{array}$ & $\begin{array}{l}\text { CAST IRON } \\
\text { BRONZE } \\
\text { CAST IRON }\end{array}$ & $\begin{array}{l}\text { BRONZE } \\
\text { BRONZE } \\
\text { BRONZE }\end{array}$ \\
\hline $\begin{array}{l}\text { BEARING FRAME } \\
\text { BEARINGS }\end{array}$ & $\begin{array}{l}\text { CAST IRON } \\
\text { BALL }\end{array}$ & $\begin{array}{l}\text { CAST IRON } \\
\text { BALL }\end{array}$ & $\begin{array}{l}\text { CAST IRON } \\
\text { BALL }\end{array}$ \\
\hline $\begin{array}{l}\text { SHAFT } \\
\text { SHAFT SLEEVE }\end{array}$ & $\begin{array}{l}\text { CARBON STEEL } \\
\text { ST. STEEL }\end{array}$ & $\begin{array}{l}\text { C.ANBON STEEL } \\
\text { COPPEH }\end{array}$ & ST. STEEL \\
\hline $\begin{array}{l}\text { MECH. SEAL } \\
\text { SEAL RING } \\
\text { SEAT INSERT } \\
\text { INSERT GASKET }\end{array}$ & $\begin{array}{l}\text { STEEL-FITTED } \\
\text { CARBON } \\
\text { CERAMIC } \\
\text { RUBBER }\end{array}$ & $\begin{array}{l}\text { BRASS - FITTED } \\
\text { CARHOH! } \\
\text { CERAM IC } \\
\text { RUBBES }\end{array}$ & $\begin{array}{l}\text { BRASS - F ITTED } \\
\text { CARIBON } \\
\text { CFAMAIIC } \\
\text { RUBBER }\end{array}$ \\
\hline BASEPLATE & STEEL & STEEL & STEEL \\
\hline 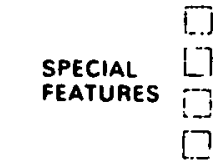 & $\begin{array}{l}\text { HIGH TEMPERAT } \\
\text { CAST IRON BAS } \\
\text { CASE WEARING }\end{array}$ & $\begin{array}{l}\text { IRE CONSTRUCTIO } \\
\text { PLATE } \\
\text { INGS }\end{array}$ & \\
\hline
\end{tabular}

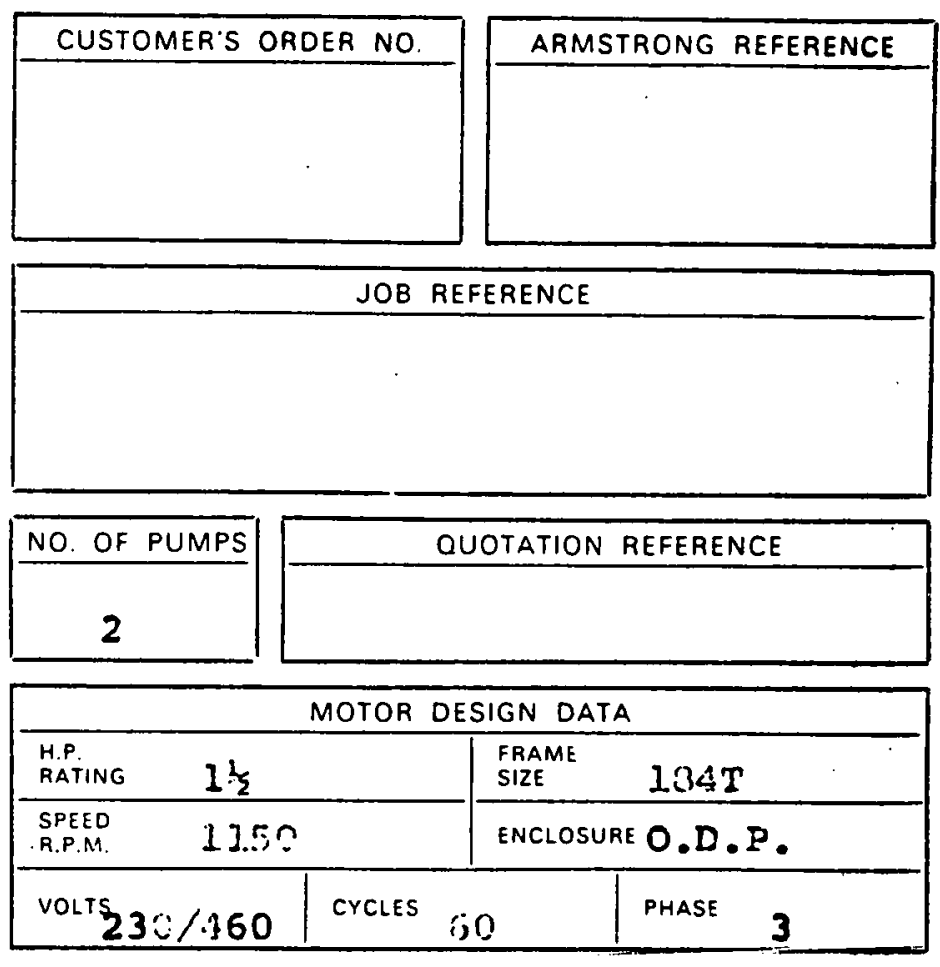

\begin{tabular}{l} 
SPECIFICATIONS \\
\hline PUMP: - \\
IIORIZONTAL SHAFT, SINGLE STAGE, END SUCTION. \\
RAOIALLY SPLIT CASING, CENTRIFUGAL TYPE WITH SINGLE \\
MECHANICAL SENL AND ANT I FAICTION BEARINGS. DI - \\
RECTLY CONNECTED TO DRIVING MOTOR THROUGH A \\
FLEXIBLE COUPLING PUMP AND MOTOR MOUNTED ON A \\
COMBINATION TYPE BASEPLATE. \\
MOTOR:- \\
MORIZONTAL SHAFT, FOOT-MOUNTED, CONTINUOUSLY RATED. \\
BALL BEARING, SQUIRREL-CAGE INDUCTION TYPE.
\end{tabular}

PREPARED BY:

DATE :

Form No. 6205 Litho in Canada REMARKS AND/OR ACCESSORIES

DIMENSIONS: SEE FILE NO.

ATTACHED

PERFORMANCE: SEE FILE NO.

ATTACHED 


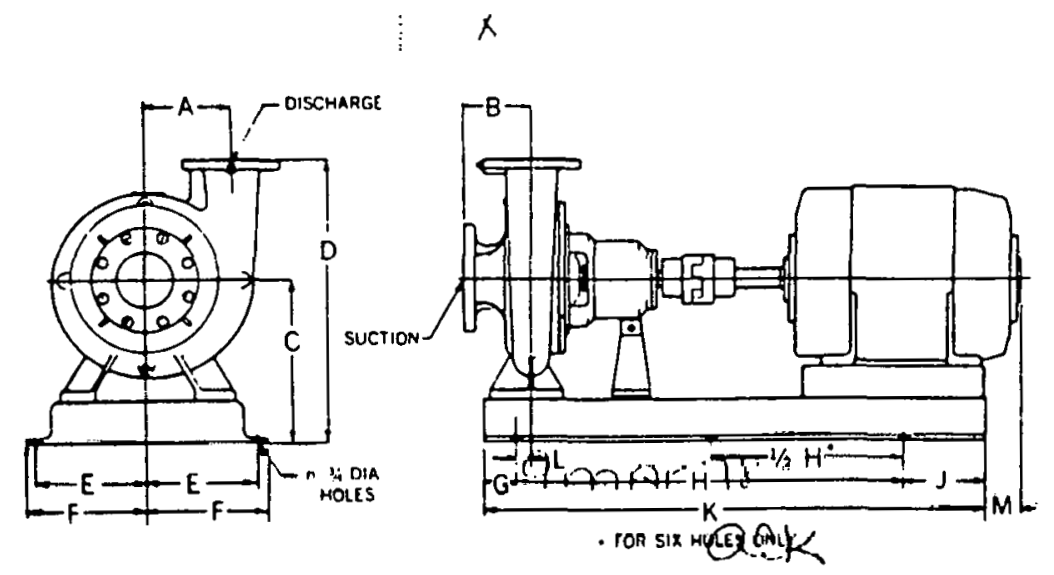

ALL DIMENSIONS IN INCHES*

\section{DIMENSIONAL DATA}

\section{SERIES 4023 AND 4030}

\section{WITH T-FRAME MOTOR}

PUMP SIZES 2" AND LARG̈ER HAVE T25 LB. STANDARD FLANGES. SMALLER PUMP SIZES HAVE SC:REWED CONNECTIONS. ALL PUMPS ARE RATED FOR 175 PSIG MAXIMUM WORKING PRESSURE.

\begin{tabular}{|c|c|c|c|c|c|c|c|c|c|c|c|c|c|c|c|c|c|c|c|c|c|}
\hline \multirow{2}{*}{$\begin{array}{l}\text { PUMP } \\
\text { SIZE }\end{array}$} & \multicolumn{2}{|c|}{$\begin{array}{l}\text { BRANCH } \\
\text { SIZES }\end{array}$} & \multicolumn{2}{|c|}{$\begin{array}{l}\text { MOTOR } \\
\text { FRAME SIZE }\end{array}$} & \multirow{2}{*}{$\begin{array}{l}\text { BASE } \\
\text { NO. }\end{array}$} & \multirow[t]{2}{*}{ A } & \multirow[t]{2}{*}{$B$} & \multirow[t]{2}{*}{$c$} & \multirow[t]{2}{*}{$D$} & \multirow[t]{2}{*}{$E$} & \multirow[t]{2}{*}{$F$} & \multirow[t]{2}{*}{$G$} & \multirow[t]{2}{*}{$\mathrm{H}$} & \multirow[t]{2}{*}{ J } & \multirow[t]{2}{*}{ K } & & \multirow[t]{2}{*}{$n$} & \multicolumn{4}{|c|}{$\begin{array}{l}\text { DIMENSION ' } M \text { '. } \\
\text { DRIP.PROOF | TEFC }\end{array}$} \\
\hline & DISCH. & suct. & (i) & (ii) & & & & & & & & & & & & & & (i) & (ii) & (i) & (ii) \\
\hline \multirow{4}{*}{$11 / \propto D$} & & & 56 & - & 1 & $41 / 2$ & $27 / 8$ & $81 / 2$ & $13 \frac{1}{2}$ & 7 & 8 & 3 & 20 & 5 & 29 & $1 \%$ & 4 & - & - & - & - \\
\hline & $1 \%$ & $11 / 2$ & 'i43T & $145 \mathrm{~T}^{\circ}$ & $s-i$ & $4 \frac{1}{2}$ & $2 \%$ & $81 / 2$ & $\overline{131 / 2}$ & 7 & $\because$ & 1 & 2 & & 29 & $11 / 8$ & 4 & - & - & $3 / 8$ & $13 / 8$ \\
\hline & NPT & NPT & $182 \mathrm{~T}$ & $184 \mathrm{~T}$ & s-3 & $4 \frac{1}{2}$ & $27 / 8$ & $81 / 2$ & $131 / 2$ & 7 & 8 & 3 & 23 & 7 & 33 & $1 y^{\prime}$ & 4 & - & 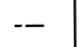 & - & - \\
\hline & & & $213 T$ & $215 \mathrm{~T}^{-}$ & S.3 & $4 \frac{1}{2}$ & $2 \%$ & $\overline{91 / 4}$ & $141 / 4$ & 7 & 8 & 3 & 23 & 7 & 33 & $1 \%$ & 4 & $3 / 4$ & $2 \frac{1}{4}$ & 31/ & $4 \%$ \\
\hline \multirow{4}{*}{$11 / 2 D$} & & & 56 & 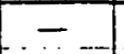 & $S-1$ & $45 / 8$ & $31 / 8$ & $81 / 2$ & $141 / 2$ & 7 & 8 & 3 & 20 & 6 & 29 & 11/i6 & 4 & - & $\cdots$ & - & - \\
\hline & $1 \frac{1}{2}$ & & $143 \mathrm{~T}$ & $145 \mathrm{~T}$ & $s-1$ & $45 \%$ & $3 \%$ & $81 / 2$ & $141 / 2$ & 7 & 8 & 3 & 20 & $\epsilon$ & 19 & $1 / 16$ & 14 & - & - & $3 / 8$ & $1 \%$ \\
\hline & NPT & NPT & $182 T$ & $184 \mathrm{~T}$ & $5-3$ & 45 & $3 \%$ & $81 / 2$ & $14 \frac{11 / 2}{2}$ & 7 & 8 & 3 & 23 & 7 & 33 & $1 \mathrm{l} / \mathrm{is}$ & 4 & - & - & - & - \\
\hline & & & $213 \mathrm{~T}$ & $215 \mathrm{~T}$ & $s-3$ & $4 \%$ & $3 \%$ & $91 / 4$ & $151 \%$ & 7 & 8 & 3 & 23 & 7 & 33 & $11 / 16$ & 4 & $3 / 4$ & $2 \%$ & $31 \%$ & $4 \%$ \\
\hline \multirow{5}{*}{ 2D } & & & 56 & - & S-1. & $43 / 4$ & 3 & $81 / 2$ & 15 & . & 8 & 3 & 20 & 6 & 29 & $11 / 16$ & 4 & - & 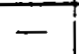 & - & - \\
\hline & & & $143 \mathrm{~T}$ & i45T & S. 1 & $43 / 4$ & 3 & $81 / 2$ & 15 & 7 & 8 & 0 & 20 & 0 & 29 & $11 / 16$ & 4 & - & - & $3 / 8$ & $13 / 8$ \\
\hline & 2 & $21 / 2$ & $182 \mathrm{~T}$ & $184 \mathrm{~T}$ & S.3 & $43 / 4$ & 3 & $81 / 2$ & 15 & 7 & 8 & 3 & 2 & 7 & 33 & $11 / 16$ & 4 & - & - & - & - \\
\hline & & & $213 \mathrm{~T}$ & $215 T$ & S-3 & $43 \%$ & 3 & $9 \frac{1}{4}$ & 153 & 7 & 8 & 3 & $2:$ & 7 & 33 & $11 / 16$. & 4 & $3 / 4$ & $2^{1 / 4}$ & $3 \%$ & $43 \%$ \\
\hline & & & $254 T$ & $256 \mathrm{~T}$ & $M-1$ & $4 \%$ & 3 & 11 & $171 / 2$ & 9 & 10 & 3 & 36 & $7 \%$ & $46 \%$ & $2 \frac{1}{116}$ & 6 & - & - & - & $1 \frac{1}{2}$ \\
\hline & & & 56 & - & $S-1$ & $411 / 16$ & $3 \%$ & $8 \frac{1}{2}$ & $14 \frac{1}{2}$ & 7 & 8 & 3 & 20 & 6 & 29 & 1 & 4 & - & - & $\overline{-}$ & $\ldots$ \\
\hline & & & $143 T$ & $145 \mathrm{~T}$ & $5-1$ & $41 / 16$ & $3 \%$ & $\overline{81 / 2}$ & $\overline{141 / 2}$ & 7 & 8 & 3 & 2 & 6 & 29 & 1 & 4 & -1 & - & $3 / 8$ & $13 / 8$ \\
\hline $21 / 2 D$ & $2 \frac{1}{2}$ & 3 & $182 \mathrm{~T}$ & $184 \mathrm{~T}$ & $5-3$ & 4 'ins & 35 & $81 / 2$ & $14 \frac{1}{2}$ & 7 & 8 & 3 & 23 & 7 & 33 & 1 & 4 & - & - & - & - \\
\hline & & ASA & $213 \mathrm{~T}$ & $215 T$ & $s-3$ & $41 \overline{1} 16$ & $3 \%$ & $91 / 4$ & $151 / 4$ & 7 & 8 & 3 & 23 & 7 & 33. & 1 & 4 & $3 / 4$ & $2 \frac{11 / 4}{4}$ & $31 / 8$ & $43 / 8$ \\
\hline & & & $254 \mathrm{~T}$ & $256 \mathrm{~T}$ & $M-1$ & 411/16 & $3 \%$ & 11 & 17 & 9 & 10 & 3 & 36 & $7 \%$ & $46 \%$ & 2 & 6 & - & $=$ & - & $1 \frac{1}{2}$ \\
\hline & & & $143 T$ & $14.5 \mathrm{~T}$ & $s-1$ & 5 & $4 \%$ & $81 / 2$ & $14 \frac{1}{2}$ & 7 & 8 & 3 & 20 & 6 & 29 & $13 / 16$ & 4 & - & $=$ & $3 / 8$ & $1 \%$ \\
\hline & & & $182 \mathrm{~T}$ & $184 T$ & $5-3$ & 5 & $4 \frac{1}{8}$ & $81 / 2$ & $14 \frac{1}{2}$ & 7 & 8 & 3 & 23 & 7 & 33 & $13 / 16$ & 4 & - & 二 & - & - \\
\hline 30 & 3 & 4 & $213 \mathrm{~T}$ & $2 i \overline{5}$ & $5-3$ & 5 & $4 \%$ & $91 / 4$ & $15 \frac{1}{4}$ & 7 & 8 & 3 & 23 & 7 & 33 & $13 / 16$ & 4 & $3 / 4$ & $21 / 4$ & $31 / 8$ & $4 \%$ \\
\hline & & & $254 \mathrm{~T}$ & $256 T$ & $M-1$ & 5 & $4 \%$ & 11 & 17 & 9 & 10 & 3 & 36 & $7 \%$ & $46 \%$ & $113 / 16$ & 6 & -1 & - & -1 & $91 / 2$ \\
\hline & & & $284 \mathrm{TS}$ & $286 \mathrm{TS}$ & $M-1$ & 5 & $4 \%$ & 11 & 17 & 9 & 10 & 3 & 36 & $7 \%$ & $46 \%$ & $113 / 16$ & 6 & $=$ & $3 / 4$ & $13 / 4$ & $31 / 4$ \\
\hline & & & $143 T$ & $145 T$ & S- & 5 & $43 / 4$ & $9 \frac{1}{2}$ & $15^{3 / 4}$ & 7 & 8 & 3 & 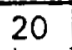 & 6 & 29 & $9 / 16$ & 4 & $=$ & $\bar{\cdots}$ & $3 / 8$ & $1 \%$ \\
\hline & & & $182 T$ & $184 T$ & $s-3$ & 5 & $43 / 4$ & $91 / 2$ & $153 / 4$ & 7 & 8 & 3 & 2 & 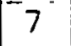 & 33 & $9 / 16$ & 44 & - & - & - & - \\
\hline & 4 & 5 & $213 T$ & $215 T$ & S-3 & 5 & $4 \%$ & $91 / 2$ & $15 \%$ & 7 & 8 & 3 & 23 & 7 & 33 & $9 / 16$ & 4 & $3 / 4$ & $2 \%$ & $31 / 8$ & $43 / 8$ \\
\hline 4 & A. & ASA & $254 \mathrm{~T}$ & $256 \mathrm{~T}$ & $M-1$ & 5 & $43 / 2$ & 12 & $18 \frac{1}{4}$ & $9^{-}$ & 10 & 3 & 36 & $7 \%$ & $46 \%$ & $19 \%$ & 6 & -1 & - & - & $1 \frac{1}{2}$ \\
\hline & & & $284 T S$ & $286 \mathrm{TS}$ & $M-1$ & 5 & $4 \%$ & 12 & $18 \frac{1}{4}$ & 9 & 10 & 3 & 36 & $7 \%$ & $461 / 4$ & $1 \% / 16$ & 6 & - & $3 / 4$ & $13 / 4$ & $31 / 4$ \\
\hline & & & $324 \mathrm{TS}$ & - & $M-1$ & 5 & $4 \%$ & 12 & $18 \%$ & 9 & 10 & 3 & 36 & $7 \%$ & $46 \%$ & $19 \%$ & 6 & $1 \%$ & - & $4 \%$ & - \\
\hline & & & $143 \mathrm{~T}$ & $145 T$ & $s-1$ & $51 / 2$ & $2^{13 / 16}$ & $91 / 2$ & $171 / 2$ & - & 8 & 3 & 20 & 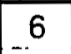 & 29 & $1 \mathrm{~V} / 6$ & 4 & -1 & $=$ & $3 / 8$ & $13 \%$ \\
\hline & & & $182 \mathrm{~T}$ & $184 \mathrm{~T}$ & $S-3$ & $51 / 2$ & $2^{13} / 16$ & $91 / 2$ & $171 / 2$ & 7 & 8 & 3 & 23 & 7 & 33 & $11 / 16$ & 4 & - & $=$ & - & - \\
\hline $11 / 4 \mathrm{E}$ & $1 \frac{11 / 4}{4}$ & $11 / 2$ & $213 \mathrm{~T}$ & $215 T$ & s. 3 & $51 / 2$ & $2^{13 / 16}$ & $91 / 2$ & $17 \frac{1}{2}$ & 7 & 8 & 3 & 23 & 7 & 33 & $11 / 16$ & 4 & $3 / 4$ & $21 / 4$ & $31 / 8$ & $43 / 8$ \\
\hline & & & $254 \mathrm{~T}$ & $256 \mathrm{~T}$ & $M-1$ & $5 \frac{1}{2}$ & $2^{13 / 16}$ & 12 & 20 & 9 & -10 & 3 & 36 & $71 / 4$ & $461 / 4$ & $2 \%$ & 6 & - & - & - & $1 \frac{1}{2}$ \\
\hline & & & $28 \overline{4 T S}$ & $286 \mathrm{TS}$ & $M-1$ & $5 \ddot{1} / 2$ & $2^{13 / 16}$ & 12 & $20^{\circ}$ & 9 & 10 & 3 & 36 & $71 / 4$ & $46^{1 / 4}$ & $2 \%$ & 6 & -1 & $3 / 4$ & $13 / 4$ & $31 / 4$ \\
\hline
\end{tabular}


.LL DIMENSIONS IN INCHES*

\begin{tabular}{|c|c|c|c|c|c|c|c|c|c|c|c|c|c|c|c|c|c|c|c|c|c|}
\hline \multirow{2}{*}{$\begin{array}{l}\text { PUMP } \\
\text { SIZE }\end{array}$} & \multicolumn{2}{|c|}{$\begin{array}{l}\text { BRANCH } \\
\text { SIZES }\end{array}$} & \multicolumn{2}{|c|}{$\begin{array}{c}\text { MOTOR } \\
\text { FRAME SIZE } \\
\end{array}$} & \multirow{2}{*}{$\begin{array}{l}\text { BASE } \\
\text { NO. }\end{array}$} & \multirow{2}{*}{$A$} & \multirow{2}{*}{ B } & \multirow{2}{*}{$C$} & \multirow{2}{*}{$D$} & \multirow{2}{*}{$E$} & \multirow{2}{*}{$F$} & \multirow{2}{*}{$G$} & \multirow{2}{*}{$\mathrm{H}$} & \multirow{2}{*}{$J$} & \multirow{2}{*}{ K } & \multirow{2}{*}{$L$} & \multirow{2}{*}{$n$} & \multicolumn{4}{|c|}{$\begin{array}{l}\text { DIMENSION } M \\
\text { ORIP-PROOF }\end{array}$} \\
\hline & DISCH. & Suct. & (i) & (ii) & & & & & & & & & & & & & & & (ii) & $\frac{\pi}{(i)}$ & (iii) \\
\hline \multirow{5}{*}{$1 \frac{1 / 2 E}{}$} & \multirow{5}{*}{$\begin{array}{l}1 \frac{1}{2} \\
\text { NPT }\end{array}$} & \multirow{5}{*}{$\begin{array}{c}2 \\
\text { NPT }\end{array}$} & $143 \mathrm{~T}$ & $145 \mathrm{~T}$ & $S-1$ & $5 \frac{1}{4}$ & $3 \%$ & $91 / 2$ & 16 & 7 & 8 & 3 & 20 & 6 & 29 & $11 / 16$ & 4 & - & - & $3 / 8$ & $13 / 8$ \\
\hline & & & $182 \mathrm{~T}$ & $184 \mathrm{~T}$ & S-3 & $5 \%$ & $3 \%$ & $9 \frac{1 / 2}{2}$ & 16 & 7 & 8 & 3 & 23 & 7 & 33 & $11 / 16$ & 4 & - & $\ddot{-}$ & - & $\ddot{-}$ \\
\hline & & & $213 \mathrm{~T}$ & $215 T$ & $5-3$ & $5 \%$ & $31 / 8$ & $91 / 2$ & 16 & 7 & 8 & 3 & $\overline{2} 3$ & 7 & 33 & $11 / 16$ & 4 & $3 / 4$ & $2 \%$ & $31 \%$ & $43 / 8$ \\
\hline & & & $254 \mathrm{~T}$ & $256 \mathrm{~T}$ & $M-1$ & $5 \%$ & $3 \%$ & 12 & $18 \%$ & 9 & 10 & 3 & 36 & $7 \ddot{\%}$ & $46 \%$ & $21 / 16$ & 6 & - & - & - & $1 \%$ \\
\hline & & & $284 \mathrm{TS}$ & $286 \mathrm{TS}$ & $M-1$ & 53 & $31 / 6$ & 12 & $18 \frac{1}{2}$ & 9 & 10 & 3 & 36 & $7 \%$ & $46 \%$ & $21 / 16$ & 6 & - & $3 / 4$ & $13 / 4$ & $31 / 4$ \\
\hline & & & $143 \mathrm{~T}$ & $145 \mathrm{~T}$ & S-1 & $5 \%$ & $37 / 16$ & $91 / 2$ & $161 / 2$ & 7 & 8 & 3 & 20 & 6 & 29 & $15 / 16$ & 4 & - & - & $3 / 8$ & $13 / 8$ \\
\hline & & & $182 T$ & $184 \mathrm{~T}$ & $5-3$ & $5 \%$ & $37 / 16$ & $91 / 2$ & $\overline{16 / 2}$ & 7 & 8 & 3 & $23^{\circ}$ & 7 & 33 & $i 3 / 16$ & 4 & - & - & $\therefore$ & - \\
\hline & & $21 / 2$ & $213 \mathrm{~T}$ & $215 \mathrm{~T}$ & S-3 & $5 \%$ & $37 / 16$ & $91 / 2$ & $161 / 2$ & 7 & 8 & 3 & 23 & 7 & 33 & $15 / 16$ & 4 & - & $2 \%$ & $3 \%$ & $43 \%$ \\
\hline $2 E$ & ASA & ASA & $254 \mathrm{~T}$ & $256 \mathrm{~T}$ & $M-1$ & $5 \%$ & $37 / 16$ & 12 & 19 & 9 & 10 & 3 & 36 & $7 \%$ & $46 \%$ & $115 / 16$ & 6 & - & $\div$ & $=$ & $1 \frac{1}{2}$ \\
\hline & & & 284TS & $286 \mathrm{TS}$ & $M-1$ & $5 \%$ & $37 / 16$ & 12 & 19 & 9 & 10 & 3 & 36 & $7 \%$ & $46 \%$ & $113 / 16$ & 6 & - & $3 / 4$ & $13 / 4$ & $3 \%$ \\
\hline & & & 324TS & - & $M-1$ & $5 \%$ & 3716 & 12 & 19 & 9 & 10 & 3 & 36 & $7 \%$ & $461 / 4$ & $713 / 16$ & 6 & $1 \%$ & $=$ & $4 \%$ & - \\
\hline & & & $182 \mathrm{~T}$ & $184 \mathrm{~T}$ & S-3 & 6 & $3 \frac{1}{2}$ & $91 / 2$ & $161 / 4$ & 7 & 8 & 3 & 23 & 7 & 33 & $19 / 16$ & 4 & - & - & - & - \\
\hline $21 / 2 E$ & ASA & ASA & $213 T$ & $215 \mathrm{~T}$ & S.3 & 6 & $31 / 2$ & $91 / 2$ & $161 / 4$ & 7 & 8 & 3 & 23 & 7 & $\overline{33}$ & $15 / 16$ & 4 & $3 \%$ & $2 \overline{1}$ & $3 \%$ & $4 \overline{3}$ \\
\hline & & & $182 \mathrm{~T}$ & $184 \mathrm{~T}$ & S.3 & $61 / 8$ & $4 \frac{3}{4}$ & $91 / 2$ & 17 & 7 & 8 & 3 & 23 & 7 & 33 & $\%$ & 4 & - & - & - & - \\
\hline $3 E$ & 3 & 4 & $213 T$ & $215 \mathrm{~T}$ & S-3 & $6 \%$ & $4 \%$ & $91 / 2$ & 17 & 7 & 8 & 3 & 23 & 7 & 33 & 5 & 4 & $3 / 4$ & $2 \%$ & $3 \%$ & 43 \\
\hline & & & $254 \mathrm{~T}$ & - & $5-7$ & $61 / 8$ & $43 / 4$ & $101 / 2$ & 18 & $73 / 4$ & $83 / 4$ & 3 & 28 & 7 & 38 & $5 \%$ & 4 & - & - & - & - \\
\hline & & & - & $184 \mathrm{~T}$ & S-5 & $6 \%$ & $43 / 4$ & $10 \frac{1}{2}$ & 18 & 7 & 8 & 3 & 23 & 7 & 33 & $5 / 6$ & 4 & - & - & - & - \\
\hline $4 E$ & 4 & 5 & $213 T$ & $215 T$ & $S-5$ & $6 \%$ & $43 / 4$ & $101 / 2$ & 18 & 7 & 8 & 3 & 23 & 7 & 33 & $5 / 8$ & 4 & $3 / 4$ & $2 \%$ & $3 \%$ & $4 \%$ \\
\hline & & & $25 \wedge \mathrm{T}$ & $266 \mathrm{~T}$ & $5-7$ & $63 \%$ & $4 \%$ & $171 / 2$ & 19 & $73 / 4$ & $8 \%$ & 3 & 28 & 1 & 38 & 5 & 4 & - & 115,16 & - & 3316 \\
\hline & & & $213 T$ & $215 T$ & S.5 & $6^{15 / 16}$ & $5 \%$ & $11 \frac{1}{2}$ & $193 / 4$ & 7 & 8 & $J$ & 23 & 7 & 33 & $1 / 8$ & 4 & $3 / 4$ & $21 / 4$ & $3 \%$ & $4 \%$ \\
\hline $5 E$ & 5 & 6 & $254 \mathrm{~T}$ & $256 \mathrm{~T}$ & S-9 & $6^{15 / 16}$ & $5 \%$ & 14 & $22 \frac{1}{4}$ & 9 & 10 & 3 & 30 & 7 & 40 & $\%$ & 4 & $=$ & $=$ & - & $13 / 16$ \\
\hline & ASA & ASA & $284 \mathrm{~T}$ & - & $M-5$ & $6^{13 / 16}$ & $5 \%$ & 14 & $22 \frac{1}{4}$ & 10 & 11 & 3 & 36 & $71 / 2$ & $46 \%$ & $1 \%$ & 6 & $1 / 6$ & - & $2 \%$ & - \\
\hline & & 8 & $254 \mathrm{~T}$ & $256 \mathrm{~T}$ & $M-3$ & $7 \frac{1}{2}$ & 7 & 15 & 26 & 10 & 11 & 3 & 36 & $31 / 4$ & $42 \%$ & $11 / 16$ & 6 & $1 \%$ & 3㽝 & $3 \frac{1}{2}$ & $5 \%$ \\
\hline 6 & ASA & ASA & $284 \mathrm{~T}$ & $286 \mathrm{~T}$ & $M-5$ & $71 / 2$ & 7 & 15 & 26 & 10 & 11 & 3 & 36 & $7 \frac{1}{2}$ & $46^{1 / 2}$ & $11 / 16$ & $\overline{6}$ & $1 / 8$ & $\overline{15 / 8}$ & $2 \%$ & $4 \%$ \\
\hline & & 3 & $213 T$ & $215 \mathrm{~T}$ & $M-3$ & $7 \frac{1}{2}$ & $51 / 2$ & 14 & $23 \frac{1}{2}$ & 10 & 11 & 3 & 36 & $31 / 4$ & $42 \frac{1}{4}$ & 13 & 6 & - & - & - & - \\
\hline $2 \mathbf{G}$ & ASA & ASA & $254 \mathrm{~T}$ & $256 \mathrm{~T}$ & $M-3$ & $71 / 2$ & $51 / 2$ & 14 & $231 / 2$ & 10 & 11 & 3 & 36 & $31 / 4$ & $42 \%$ & $1 \%$ & 6 & - & $13 / 8$ & $13 / 4$ & $3 \%$ \\
\hline 39 & & & $213 T$ & $215 T$ & $M-3$ & $71 / 2$ & $5 \%$ & 14 & $231 / 2$ & 10 & 11 & 3 & 36 & $31 / 4$ & $42 \frac{1}{4}$ & $13 \%$ & 6 & - & - &.- & $=1$ \\
\hline $3 G$ & ASA & ASA & $254 \mathrm{~T}$ & $256 \mathrm{~T}$ & $M \cdot 3$ & $71 / 2$ & $51 / 2$ & 14 & $231 / 2$ & 10 & 11 & 3 & 36 & $3 \%$ & $42 \%$ & $1 \%$ & 6 & - & $13 / 8$ & $13 / 4$ & $3 \%$ \\
\hline & & & $213 T$ & $215 T$ & $M-3$ & 8 & 6 & 15 & 26 & 10 & 11 & 3 & 36 & $3 \%$ & $42 \frac{1}{4}$ & $11 / 4$ & 6 & - & - & - & $=$ \\
\hline 4G & 4 & 5 & $254 \mathrm{~T}$ & $256 \mathrm{~T}$ & $M-3$ & 8 & 6 & 15 & 26 & 10 & 11 & 3 & 36 & $3 \frac{1}{4}$ & $42 \%$ & $1 \%$ & 6 & - & $13 / 8$ & $13 / 4$ & $3 \frac{5}{8}$ \\
\hline & ASA & ASA & 284T & $286 \mathrm{~T}$ & $M-5$ & 8 & 6 & 15 & 26 & 10 & 11 & 3 & 36 & $71 / 2$ & $461 / 2$ & $1 \frac{1}{4}$ & 6 & - & $1 / 8$ & $11 / 6$ & $2 \%$ \\
\hline & & & $254 \mathrm{~T}$ & $256 \mathrm{~T}$ & $M-7$ & $9 \%$ & 8 & 16 & 29 & $13 \frac{1}{2}$ & $14 \frac{1}{2}$ & 6 & 36 & 6 & 48 & $5 / 16$ & 6 & - & $=$ & - & $3 / 8$ \\
\hline & & & $284 \mathrm{~T}$ & $286 \mathrm{~T}$ & $M-7$ & $9 \%$ & 8 & 16 & 29 & $131 / 2$ & $14 \frac{1}{2}$ & 6 & 36 & 6 & 48 & $3 / 16$ & 6 & $=$ & 1 & 2 & $3 \frac{1}{2}$ \\
\hline & ASA & ASA & $324 \mathrm{~T}$ & $326 \mathrm{~T}$ & $M-7$ & $91 / 4$ & 8 & 16 & 29 & $13 \frac{1}{2}$ & $14 \frac{1}{2}$ & 6 & 36 & 6 & 48 & $5 / 16$ & 6 & $21 / 2$ & 4 & $5 \%$ & $6 \%$ \\
\hline & & & $364 \mathrm{~T}$ & $365 T$ & $M-9$ & $9 \%$ & 8 & 16 & 29 & $131 / 2$ & $14 \frac{1}{2}$ & 6 & 44 & 6 & 56 & $5 / 16$ & 6 & - & $=$ & $1 \%$ & $2 \%$ \\
\hline
\end{tabular}




\section{ARMSTRONG CENTRIFUGAL PUMPS PERFORMANCE CURVES}

\section{SERIES 4030-4035-4040}
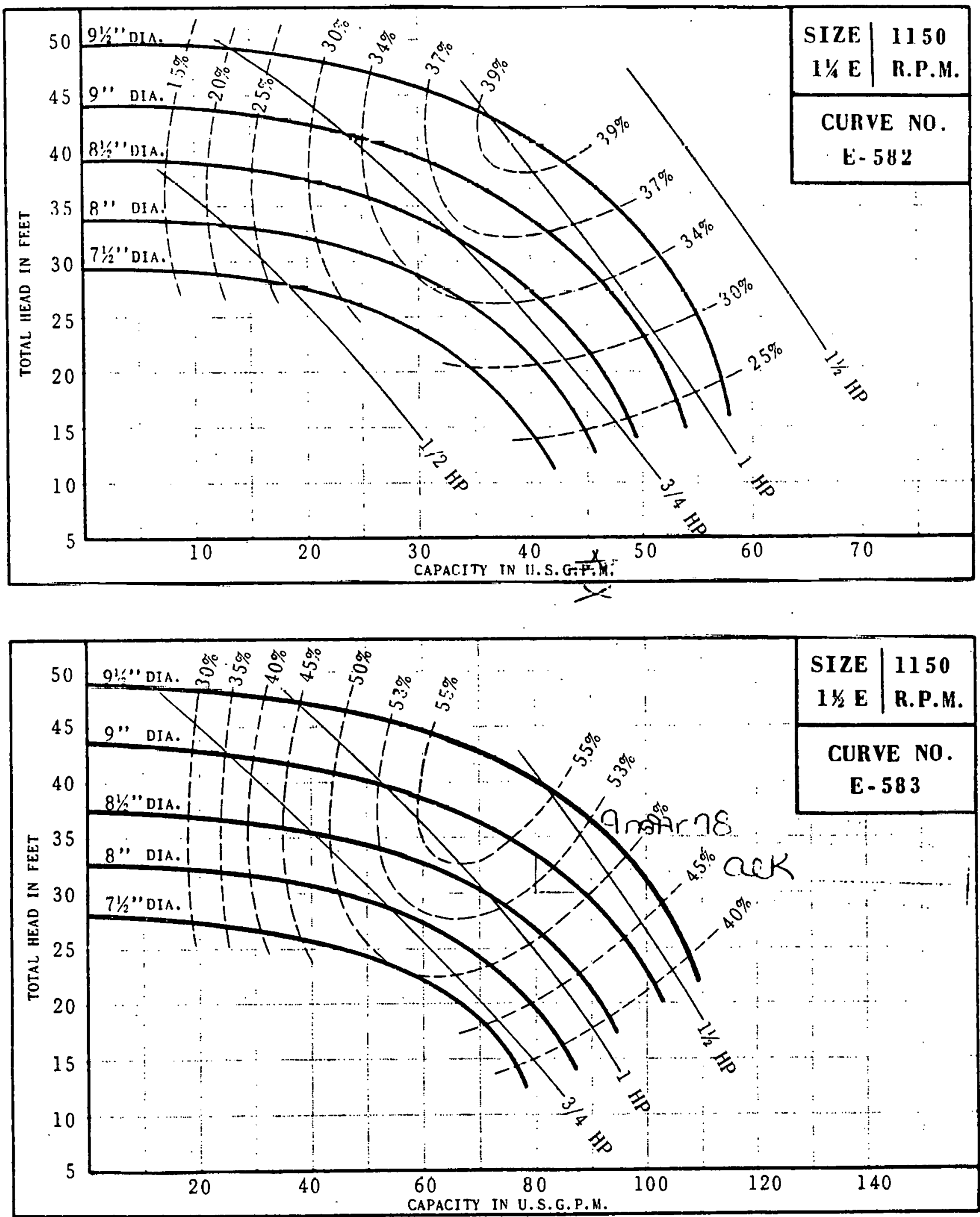


\section{SERIES 4030-4035-4040 PERFORMANCE CURVES}
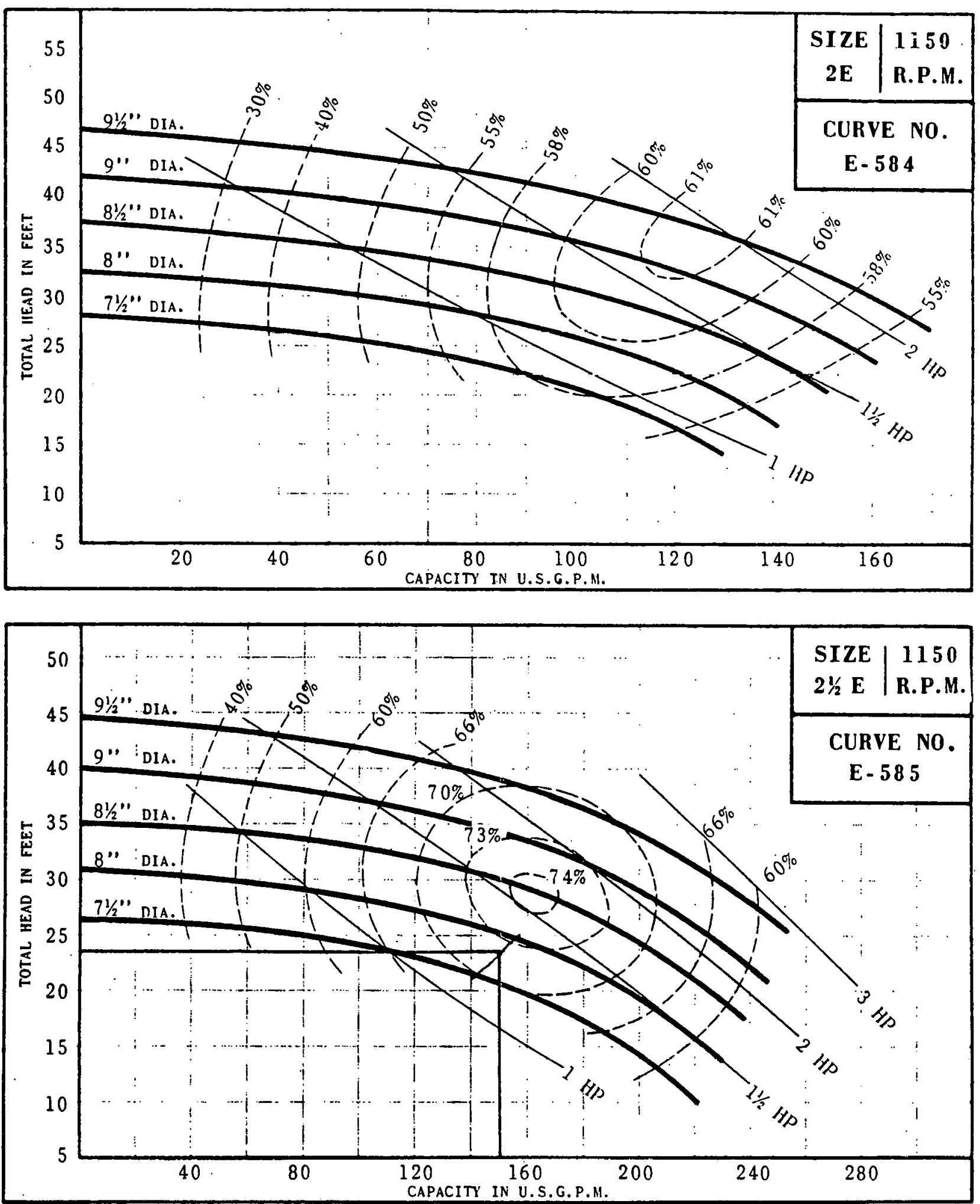


\section{ARMSTRONG CENTRIFUGAL FUMPS SUBMITTAL DATA}

\section{SERIES 4.380}

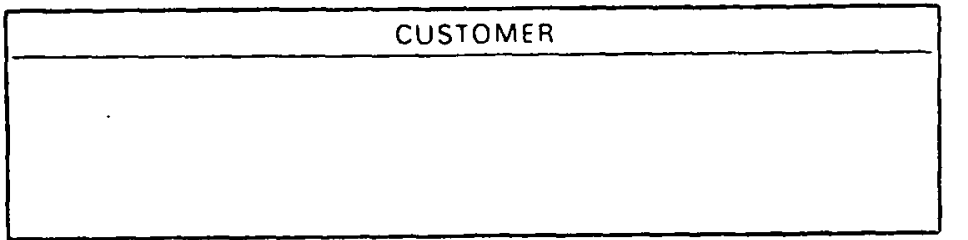

ENGINEER

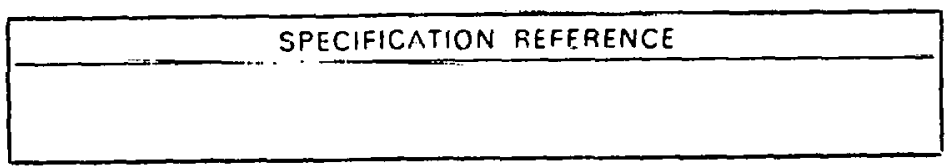

\begin{tabular}{|c|c|c|}
\hline \multicolumn{3}{|c|}{ PUMP DESIGN DATA } \\
\hline $\begin{array}{l}\text { PUMP } \\
\text { SIZE }\end{array}$ & $3-1+1$ & LMOUIO \\
\hline $\begin{array}{l}\text { CAPACITY } \\
\text { U.S.G.P.M. }\end{array}$ & 150 & $\underset{F}{\text { TFMP. }}$ \\
\hline $\begin{array}{l}\text { FEET } \\
\text { HEAD }\end{array}$ & 34 & $\begin{array}{l}\text { SPECIFIC } \\
\text { GRAVITY }\end{array}$ \\
\hline
\end{tabular}

\begin{tabular}{|c|c|c|c|}
\hline \multirow{2}{*}{$\begin{array}{l}\text { PART } \\
\text { NAME }\end{array}$} & \multicolumn{3}{|c|}{ CONSTRUCTION } \\
\hline & $\begin{array}{ll}A L L & \vdots \\
\text { IRON }\end{array}$ & $\begin{array}{l}\text { BRONZE - } \\
\text { FITTED }\end{array}$ & $\begin{array}{l}\text { ALL- } \\
\text { BRONZE }\end{array}$ \\
\hline $\begin{array}{l}\text { VOLUTF } \\
\text { IMPELLER }\end{array}$ & $\begin{array}{l}\text { CAST IHON } \\
\text { CAST IHON }\end{array}$ & $\begin{array}{l}\text { CAST IRON } \\
\text { BHONZE }\end{array}$ & $\begin{array}{l}\text { BRONZE. } \\
\text { BSTOUNZE }\end{array}$ \\
\hline $\begin{array}{l}\text { BRACKET } \\
\text { SHAFT SLEFVE }\end{array}$ & $\begin{array}{l}\text { CAST IRON } \\
\text { PLATEN STEFI }\end{array}$ & $\begin{array}{l}\text { CAST IRON } \\
\text { lWIS:ZE }\end{array}$ & $\begin{array}{l}\text { BRONZE } \\
\text { BRONZEE }\end{array}$ \\
\hline $\begin{array}{l}\text { MECII. SEAL } \\
\text { SEAL RING } \\
\text { SEAT INSERT } \\
\text { INSERT GNSKET }\end{array}$ & $\begin{array}{l}\text { STEEL FITTED } \\
\text { CARBOY } \\
\text { CERANIC } \\
\text { HUDBER }\end{array}$ & $\begin{array}{l}\text { RRASS FIT'TED } \\
\text { CARMOA } \\
\text { CEMAMIC } \\
\text { RUBRER }\end{array}$ & $\begin{array}{l}\text { BHASS F ITTED } \\
\text { CARBON } \\
\text { CERAMIC } \\
\text { RUBDER }\end{array}$ \\
\hline $\begin{array}{lc} & \vdots \\
\text { SPECIAL } & 1 \\
\text { FEATURES } & \vdots \\
& \vdots \\
& \vdots\end{array}$ & & & \\
\hline
\end{tabular}

REMARKS AND OF ACCESSORIES

DIMENSIONS: SE.T HFVFRSE SIMF. PERFORMANCE: SEE FII.E NO.
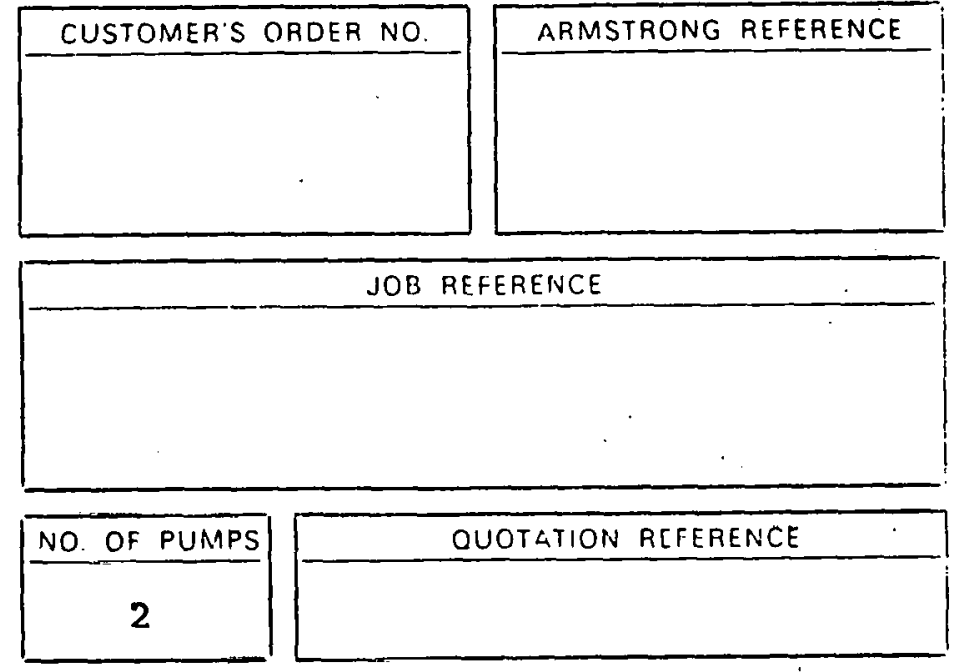

\begin{tabular}{|l|l|l|l|l|}
\hline \multicolumn{5}{|c|}{ MOTOR DESIGN DATA } \\
\hline $\begin{array}{l}\text { H.P. } \\
\text { RATING, }\end{array}$ & 2 & FMAME & \\
\hline $\begin{array}{l}\text { SIZE } \\
\text { R.P.M }\end{array}$ & 1750 & 145 & $\mathrm{JM}$ \\
\hline VOLIS & & ENCLOSURE \\
\hline
\end{tabular}

SPECIFICATIONS

PL'AII': -

IFITJCAL SIMIT, SINGIE STALIF, SINGLE SUCTION,

HAIIAH.IY STI.IT C.ASING, IN-LINE. MOUNTING CENTHIF.

URAL TYPE WITII SINGLE MEGMAICAL SEAL. IMPELLFR

MOUNTED DIRECTLY ON MOTOR SIIAF"I EXTENSION.

MOTOR: -

VERTICAL, SOLIN SHAFT, CONTINUOLSLY RITED, BALL

BEARING, SQUUTRRF.I. T.AGE, INDUCTION TYPE WITH NEMA

"C" FLANGE AND EXTENDED SHIFT.

MAXIMUM WORKING PRESSURE - PUMP VOLUTE

$\begin{array}{lll}\text { CAST IINON } & 175 \text { PSIG } & \square \\ \text { CAST INON } & 250 \text { PSIG } & \square \\ \text { CAST STEEL } & 300 \text { PSIG } & \square\end{array}$

siricial.

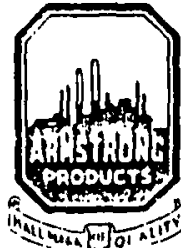




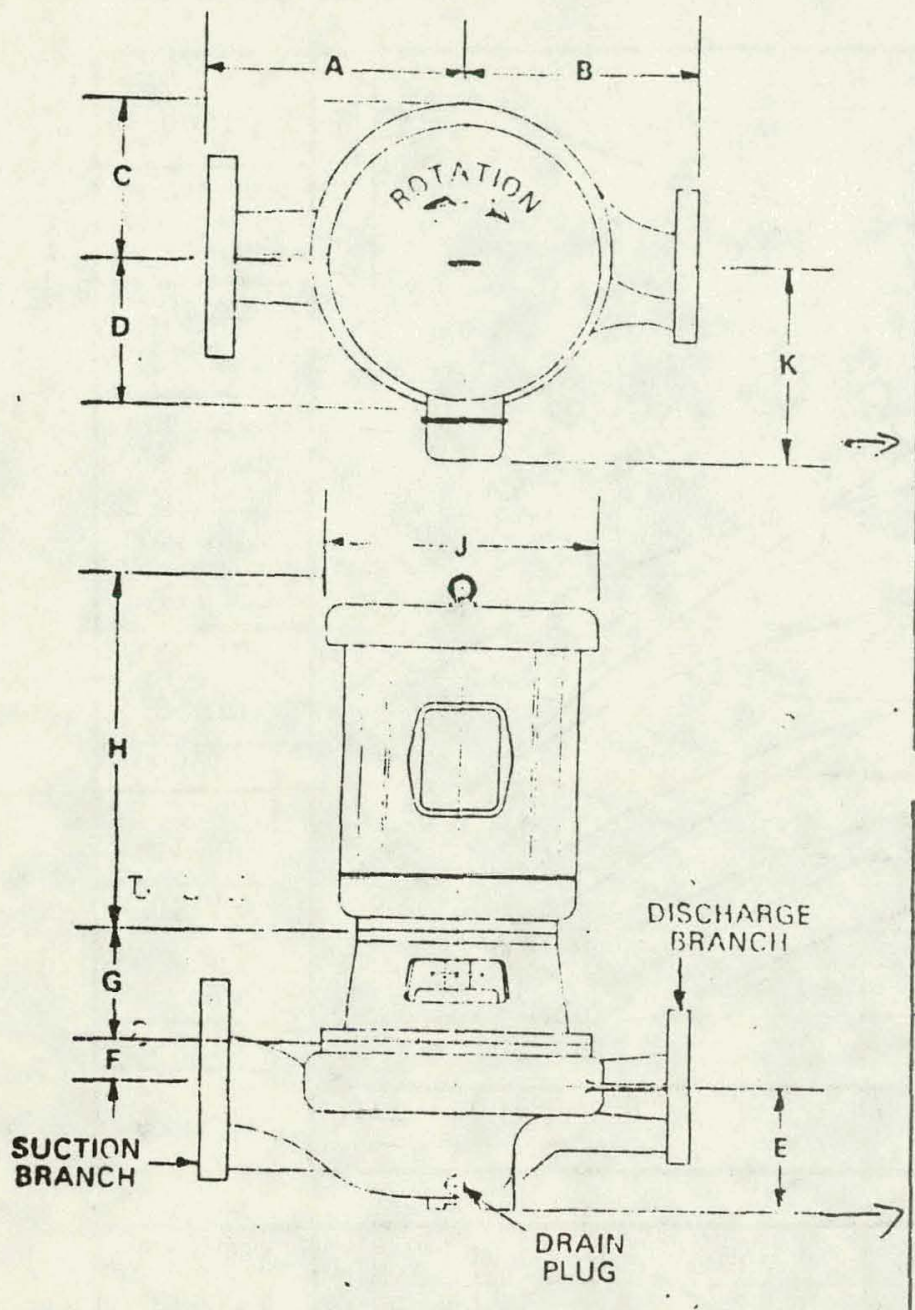

All dimensions are in inches. For exact in: tallaion diat?. please write factory for certified dinfensions.

\begin{tabular}{|c|c|c|c|c|c|c|c|c|}
\hline \multicolumn{9}{|c|}{ PUMP DIMENSIONS } \\
\hline \multirow{2}{*}{$\begin{array}{l}\text { PUMP } \\
\text { SIZE }\end{array}$} & \multicolumn{2}{|c|}{$\begin{array}{l}\text { BRANCH } \\
\text { SIZES }\end{array}$} & \multicolumn{6}{|c|}{ DIMENSIDES } \\
\hline & Disch. & Suct. & A & B. & c & $n$ & $E$ & $i$ \\
\hline $11 / 2 \mathrm{D}$ & $1^{\prime \prime} ;$ & 2 & 7 & $\sigma^{\prime}:$ & $4^{\prime}$ & $4 \%$ & $3^{2} ;$ & 1 i" \\
\hline $2 \mathrm{D}$ & 2 & $2 \% 2$ & 8 & 7 & 5 & A. & $1^{\prime}:$ & $i_{t}$ \\
\hline $3 D$ & 3 & 4 & 10 & 9 & $6^{3 / 4}$ & $\vdots$ & $\therefore$ & $1:$ \\
\hline $4 D$ & 4 & 6 & 12 & 10 & $6^{\prime}$; & 5 & 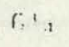 & 2 \\
\hline $11 / 2 \mathrm{E}$ & $11 / 2$ & 2 & $9^{1}=$ & 9 & $6^{\prime}$, & $5 \%$ & $\therefore$ & $1^{\prime}:$ \\
\hline $2 \mathrm{E}$ & 2 & 3 & 11 & 10 & $7: 4$ & E'. & $5^{\prime \prime}$ & $1{ }^{10}{ }_{11}$ \\
\hline $3 E$ & 3 & 4 & 12 & 11 & 7 & $E^{\circ}:$ & $5^{\prime}:$ & $1 ? 8$ \\
\hline $4 E$ & 4 & 6 & 14 & 12 & $8{ }^{\circ} \mathrm{B}$ & 63: & $2^{\prime}:$ & 2 \\
\hline
\end{tabular}

\begin{tabular}{|c|c|c|c|c|c|c|c|c|}
\hline \multicolumn{9}{|c|}{ FLANGE DETAILS } \\
\hline \multirow{3}{*}{$\begin{array}{l}\text { BRANCH } \\
\text { SIZE }\end{array}$} & \multicolumn{4}{|c|}{$\begin{array}{l}125 \text { LB ASA CAST IRON } \\
150 \text { LB ASA CAST STEEL }\end{array}$} & \multicolumn{4}{|c|}{$\begin{array}{l}250 \text { LR ASA CRET IRCN } \\
300 \text { LR ASSA SAS STELL }\end{array}$} \\
\hline & \multirow{2}{*}{$\begin{array}{l}\text { Flange } \\
\text { O.D }\end{array}$} & \multicolumn{2}{|c|}{ Bolts } & \multirow{2}{*}{ B.C.D } & \multirow{2}{*}{$\begin{array}{c}\text { Flang: } \\
\text { D. }\end{array}$} & \multicolumn{2}{|c|}{ E.rits } & \multirow{2}{*}{ PC.D } \\
\hline & & No & Sire & & & No, & sur & \\
\hline 11,2 & 5 & 4 & $1 / 2$ & $37 / 8$ & $6^{\prime} 8$ & $\therefore$ & 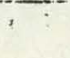 & $4^{\prime}$ \\
\hline 2 & 6 & 4 & 5 म & $4^{3}+$ & $6 \because 2$ & 3 & . & 5 \\
\hline $2 \div$ & 7 & 4 & $5 / 8$ & $5 !: 2$ & $7 \%$ & $\therefore$ & & $5 \%$ \\
\hline 3 & $71: 2$ & 4 & $5 / 8$ & 6 & $8 \%$ & $\because$ & $\therefore$ & $6:=$ \\
\hline 4 & 9 & 8 & $5 / 8$ & 712 & 10 & in & ' & $T^{\prime}:$ \\
\hline 6 & 11 & 8 & $3 / 4$ & $9 \%$ & $12 !$ & 1: & . & io : \\
\hline
\end{tabular}

\begin{tabular}{|c|c|c|c|c|c|c|c|c|c|c|}
\hline \multicolumn{11}{|c|}{ MOTOR DIMENSIONS } \\
\hline $\begin{array}{c}\text { MOTOR } \\
\text { FRAME } \\
\text { SIZE } \\
\text { SERIES } \\
4380\end{array}$ & $\begin{array}{l}H^{n} \text { (I) } \\
1 R 00 \\
\text { RIMA }\end{array}$ & $\begin{array}{l}\text { HP (a) } \\
3600 \\
\text { RPM }\end{array}$ & G & DRIF & Roof & K & G & H & C & k \\
\hline $143 \mathrm{JM}$ & 1 & $1 \%$ & $3^{3}{ }_{16}$ & 9 / $_{8}$ & 7 & $53 / 4$ & $3^{3}{ }^{3}$ & $101 / 2$ & $71 \%$ & $5^{7}$ \\
\hline $145 . J M$ & $9 \%$ & 2 & $3^{i}{ }_{1 k}$ & $10^{1 / n}$ & 7 & $53 / 4$ & $3^{3}{ }^{36}$ & $11 \%$ & $7 \overline{19}$ & $5 \gamma^{r}$ \\
\hline $145 J M$ & 2 & 3 & $3^{2}{ }_{11}$ & $i()^{1} \mathrm{in}$ & 7 & $5^{3} / 4$ & $3^{3}{ }_{16}$ & $11 \frac{1}{2}$ & $7 \because 1$ & 57 \\
\hline $182 . J M$ & 3 & 5 & $3^{\prime}$ & $10 \%$. & 9 & $81 / k$ & $3^{3}{ }_{i 6}$ & $12 \%$ & $9^{\circ}$ & $8: \ldots$ \\
\hline 184.Jn9 & 5 & $7 !$ & $3_{14}^{3}$ & $11 \% \mathrm{H}$ & 9 & $8 !$ : & $3^{3} \mathrm{in}$ & $13: \mathrm{is}$ & $83:$ & $8^{\prime}:$ \\
\hline 213.JM & $7^{\prime \prime}$ & 10 & $3^{i}{ }_{1 k}$ & $13: ;$ & $10 \%$ & $8^{3}$ & $3 i_{i n}$ & $15 \%$ & 11 & 91 \\
\hline 215JM & 10 & 15 & 3 is & $13 \%$ & $105 / 8$ & $83 / 4$ & $3^{3}$ is & $19^{3}$ i & $11 \div$ & 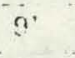 \\
\hline $254 T C Z$ & 15 & 20 & $77_{16}$ & $12: \div$ & $12 \%$ & $93 ;$ & $7 \div 16$ & $20^{\circ}$ & $1.3: 8$ & $10 \%$ \\
\hline $256 \mathrm{TC}$ & 20 & 25 & 7 ! is & $20^{\prime} ;$ & $12 \%$ & $9^{3} 4$ & $7 !$ is & $22^{2}$ & $13^{\circ}$ & $10 \%$ \\
\hline 284TC.Z & 25 & 30 & $7 ?_{16}$ & 21 & 14 & $111 / 4$ & $7 !$ is & 23 & 15 & $12^{5: n}$ \\
\hline $286 \mathrm{TCZ}$ & 30 & 40 & $7^{7} i_{i b}$ & $22^{\prime} ;$ & 14 & $111 / n$ & $7 !$ is & $24 \%$ & 15 & $12^{n}$ \\
\hline $324 \mathrm{TCZ}$ & 40 & 50 & $71 / 6$ & 23 & 16 & 14 & $7 !_{i 6}$ & 251 & 17 & 151 \\
\hline $3267 \mathrm{CZ}$ & 50 & 60 & $71 \%$ & $24 \%$ & 16 & 14 & $7 !_{16}$ & $20: 4$ & 17 & $151 \%$ \\
\hline
\end{tabular}




ARMSTRONG PERFORMANCE CURVES
SERIES 4300/80/85 $1750 \mathrm{RPM}$
Ret
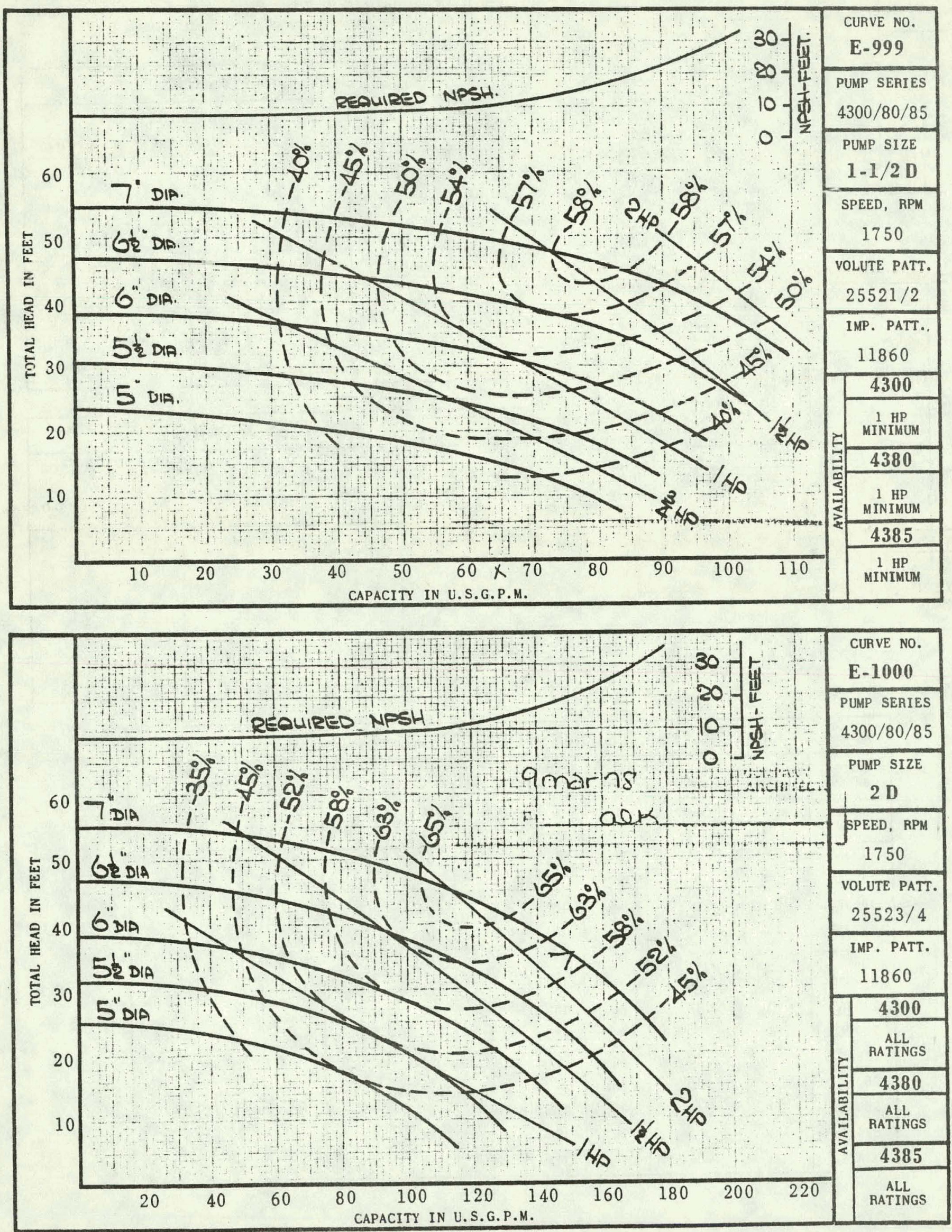

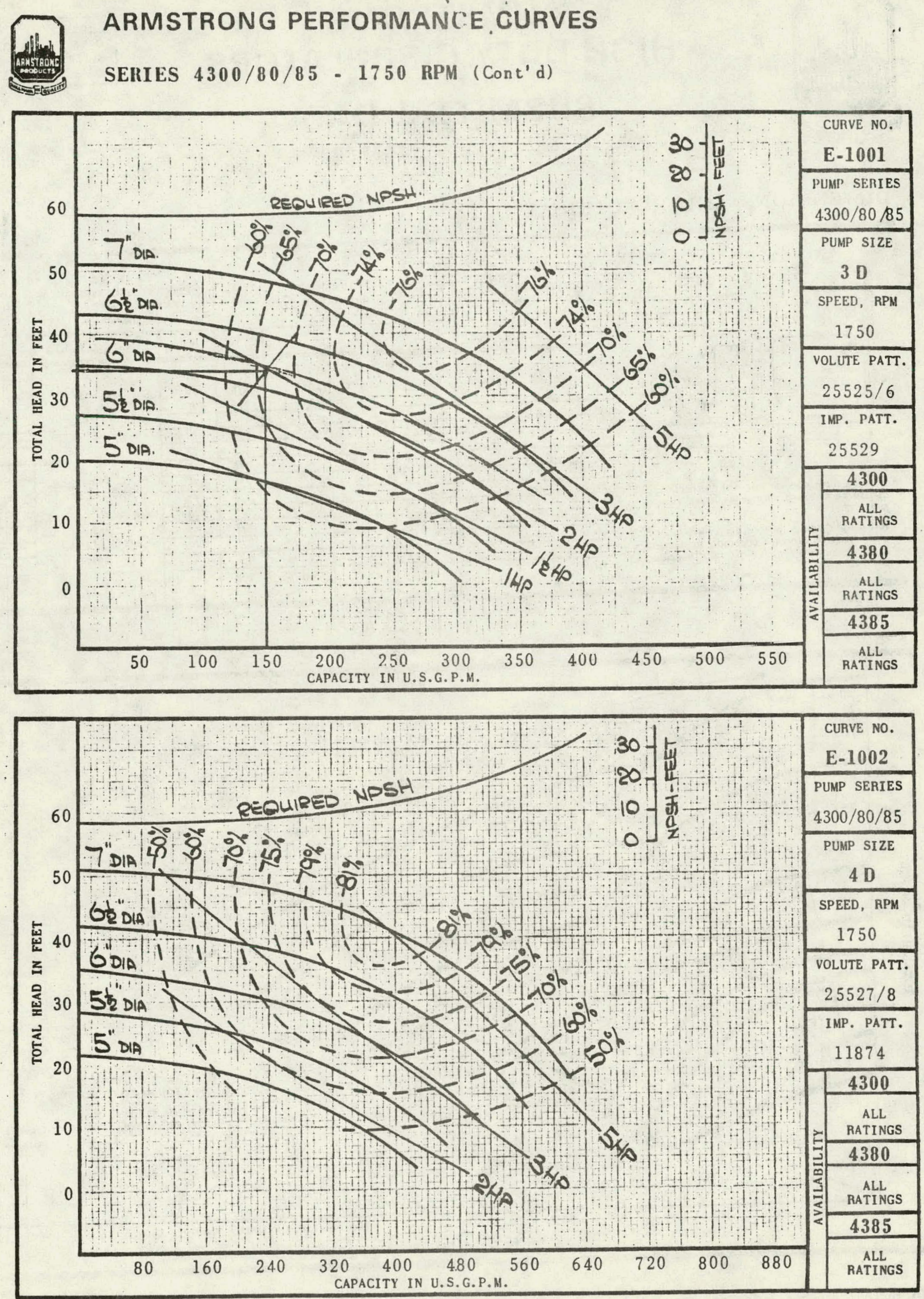


\title{
ARMST'RONG \\ HIGH DUTY CIRCULATORS \\ SUBMITTAL DATA
}

\author{
60 CYCLE - 1750 RPM
}

DIMENSIONS AND ELECTRICAL DATA

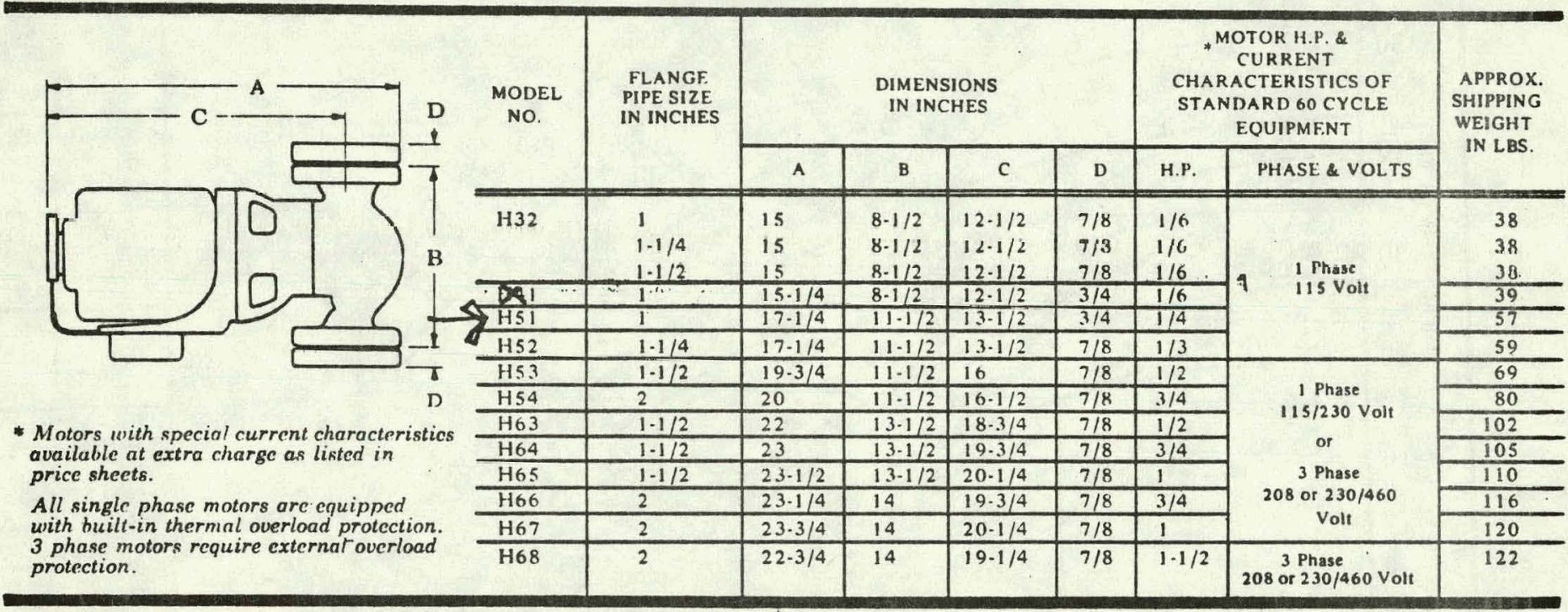

\section{appari:

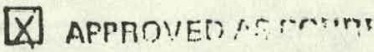

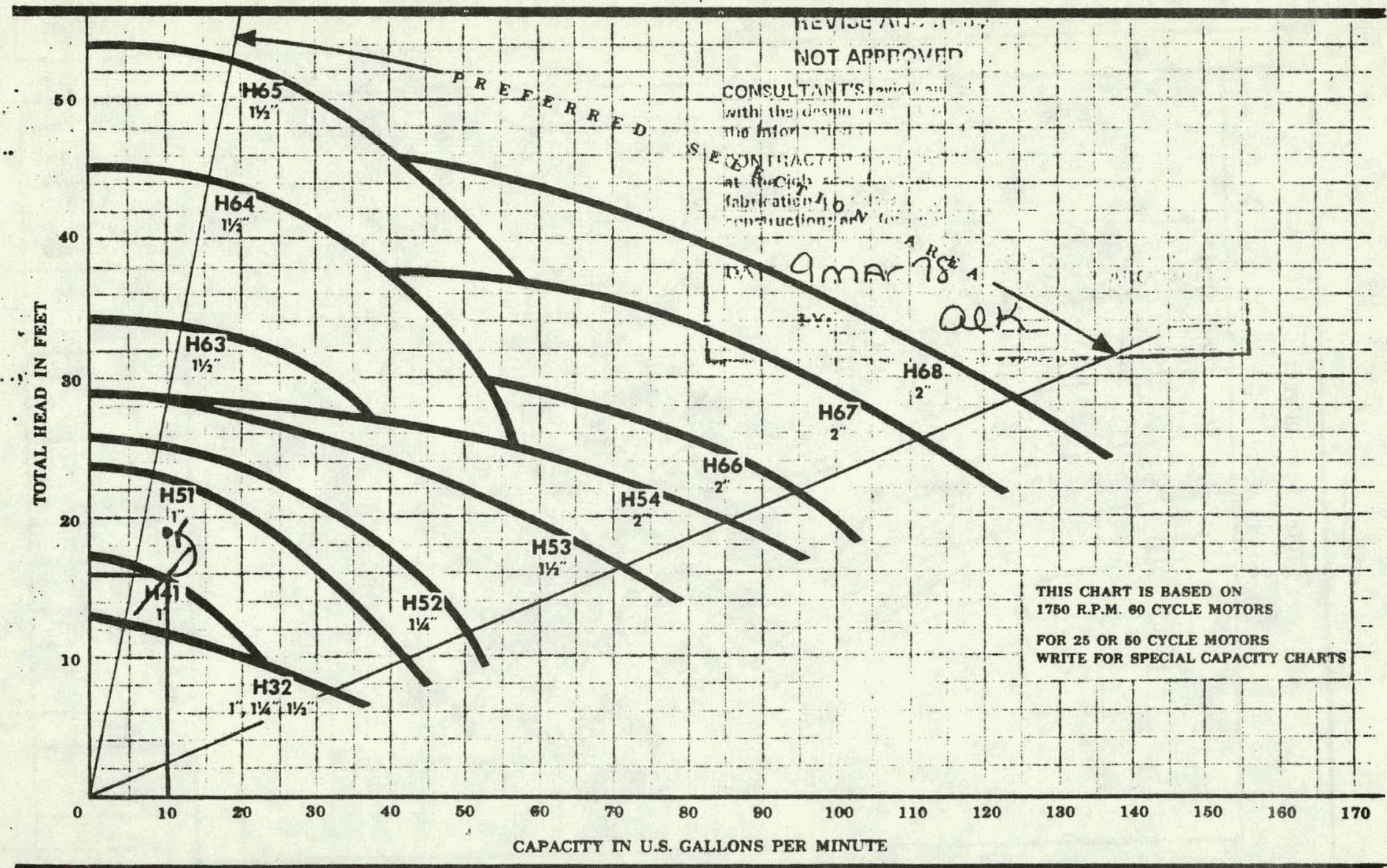

Dimensions shown are for reference only. For exact installation data, write Marketing

Division for certified dimensions. 


\section{ARMSTRONG HIGH DUTY CIRCULATORS SUBMITTAL DATA}

・nDELS: II32-II41-II51-II52-II53-II54-H63-H64-II65-II66-II67-II68 60 CYCLE 1750 RPM

\begin{tabular}{|c|}
\hline CUSTOMIEK \\
\hline FNGINEER \\
\hline SPECIFICATION REFERENCE \\
\hline PUMP DESIGN DATA \\
\hline \multirow{2}{*}{$\begin{array}{l}\text { PUMP MODEL } \\
\text { AND SIZE: } \\
\text { NOTE: Models H-32 and H-41 are down-discharge. } \\
\text { Models } \mathrm{H}-51 \text { thru H-68 are up-discharge. }\end{array}$} \\
\hline \\
\hline $\begin{array}{l}\text { TEMP. } \\
\text { OF. }\end{array}$ \\
\hline $\begin{array}{l}\text { FEET } \\
\text { HEAD } \\
\end{array}$ \\
\hline $\begin{array}{l}\text { COMPANION FLANGES ARE FURNISHED FOR SUCTION } \\
\text { AND DISCHARGE OF PUMP }\end{array}$ \\
\hline
\end{tabular}
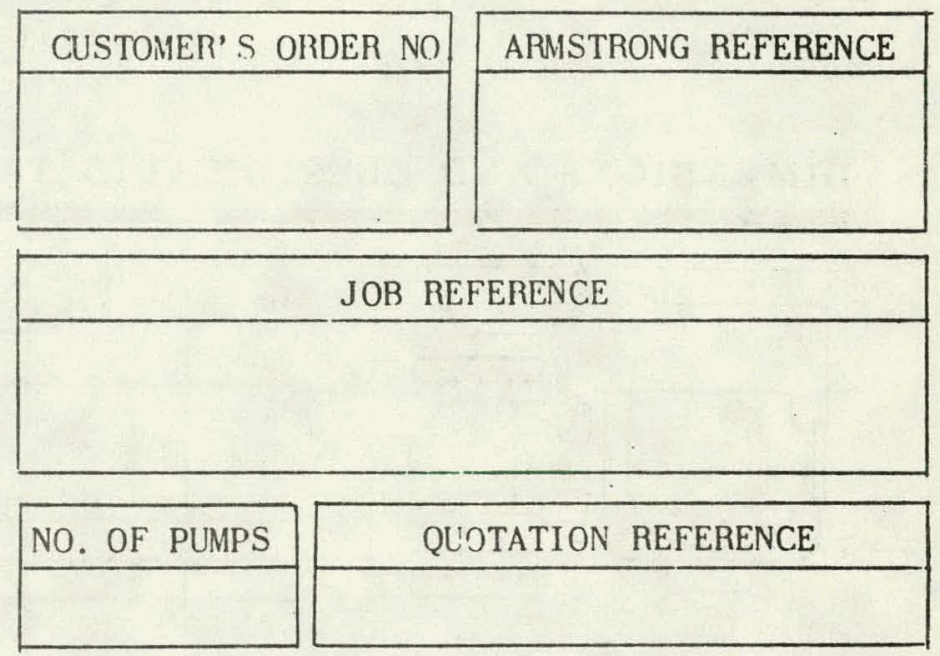

\begin{tabular}{|l|l|l|}
\hline \multicolumn{3}{|c|}{ MOTOR DESIGN DATA } \\
\hline $\begin{array}{l}\text { H.P } \\
\text { RATING }\end{array}$ & ENCLOSURE \\
\hline $\begin{array}{l}\text { SPEED } \\
\text { R.P.M. }\end{array}$ & \\
\hline VOLTS & CYCLES & PHASE \\
\hline
\end{tabular}

MAXIMUM WORKING PRESSURE: For Models $\mathrm{H}-32$ and H-41- 125 psi. For Models H-51 thru H-68 - 175 psi MAXIMUM STANDARD OPERATING TEMPERATURE: $225^{\circ} \mathrm{F}$.

\begin{tabular}{|c|c|c|}
\hline \multirow{2}{*}{ PART NAME } & \multicolumn{2}{|c|}{ STANDARD PUMP CONSTRUCTION } \\
\hline & MODELS H-32 \& H-4I & MODELS $\mathrm{H}-51$ THRU $\mathrm{H}-68$ \\
\hline & STANDARD & BRONZE FITTED \\
\hline VOLUTE CASING $\ldots \ldots \ldots \ldots$ & Cast Iron & Cast Iron \\
\hline IMPELLER $\ldots \ldots \ldots \ldots \ldots$ & Steel - Plated & $\begin{array}{l}\text { H-51 to H-54: Brass - Stamped } \\
\text { H-63 to HI-68: Bronze-Cast }\end{array}$ \\
\hline 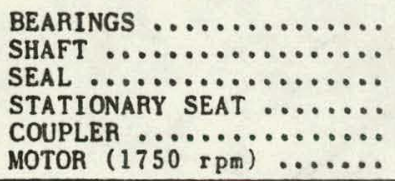 & $\begin{array}{l}\text { Sleeve, Oil Lubricated } \\
\text { Alloy Steel - Copper Sleeve } \\
\text { Mechanical } \\
\text { Cast Iron } \\
\text { Flexible, 4-Spring Type } \\
\text { Sleeve, Resilient Mounted }\end{array}$ & $\begin{array}{l}\text { Sleeve, Oil Lubricated } \\
\text { Alloy Steel - Copper Sleeve } \\
\text { Mechanical } \\
\text { ARMSEAL Coramic } \\
\text { Flexible Type } \\
\text { Sleeve, Resilient Mounted }\end{array}$ \\
\hline
\end{tabular}

\begin{tabular}{|l|}
\hline OPTIONAL FEATURES \\
\hline$\square$ HIGH TEMP. CONSTRUCTION \\
$\square$ STAINLESS STEEL SHAFT \\
$\square$ SPECIAL ELECTRICS \\
$\square$ ALL IRON CONSTRUCTION \\
$\square$ ALL BRONZE CONSTRUCTION \\
$\square$ BRONZE FITTED CONSTRUCTION \\
$\square$ SPECIAL MOTOR ENCL. \\
\hline
\end{tabular}

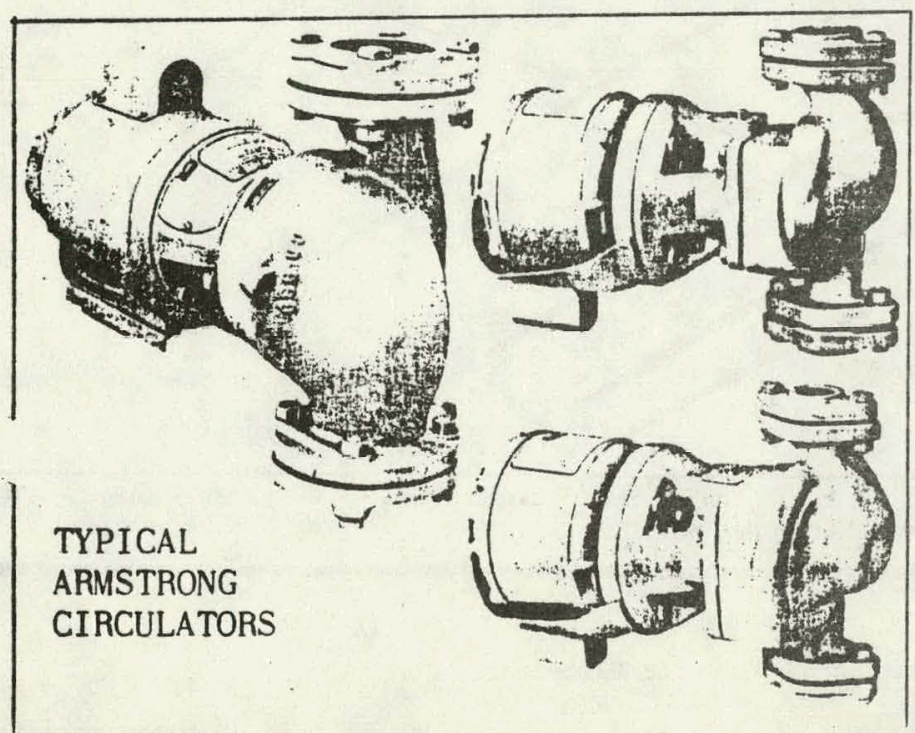

PREPARED BY:

DATE 


\title{
ARMISTRONG \\ STANDARD CIRCULATORS
}

SUBMITTAL DATA

FILE NO: $\quad 5010.90$

DATE: AUR. 15, 1973

SUPERSEDES: $\quad 5010.90$

DATE: July 30,1970

\author{
60 CYCLE - 1750 RPM
}

DIMENSIONS AND ELECTRICAL DATA

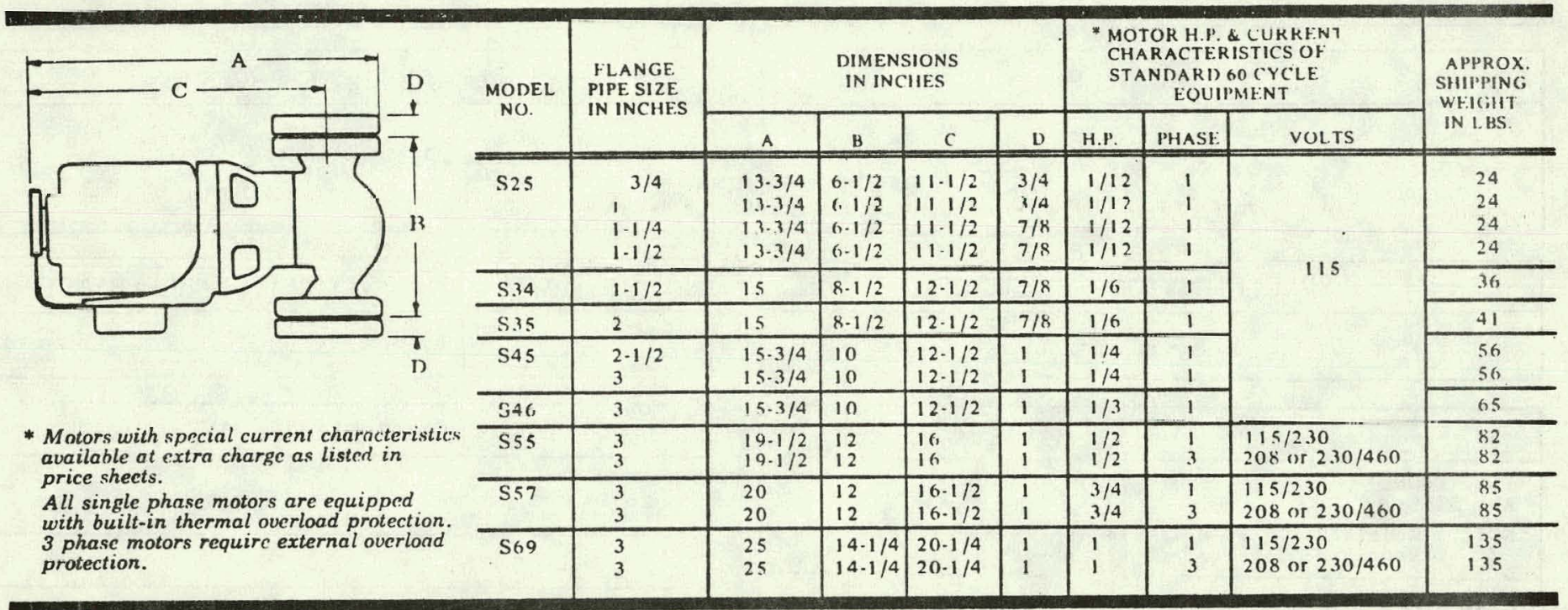

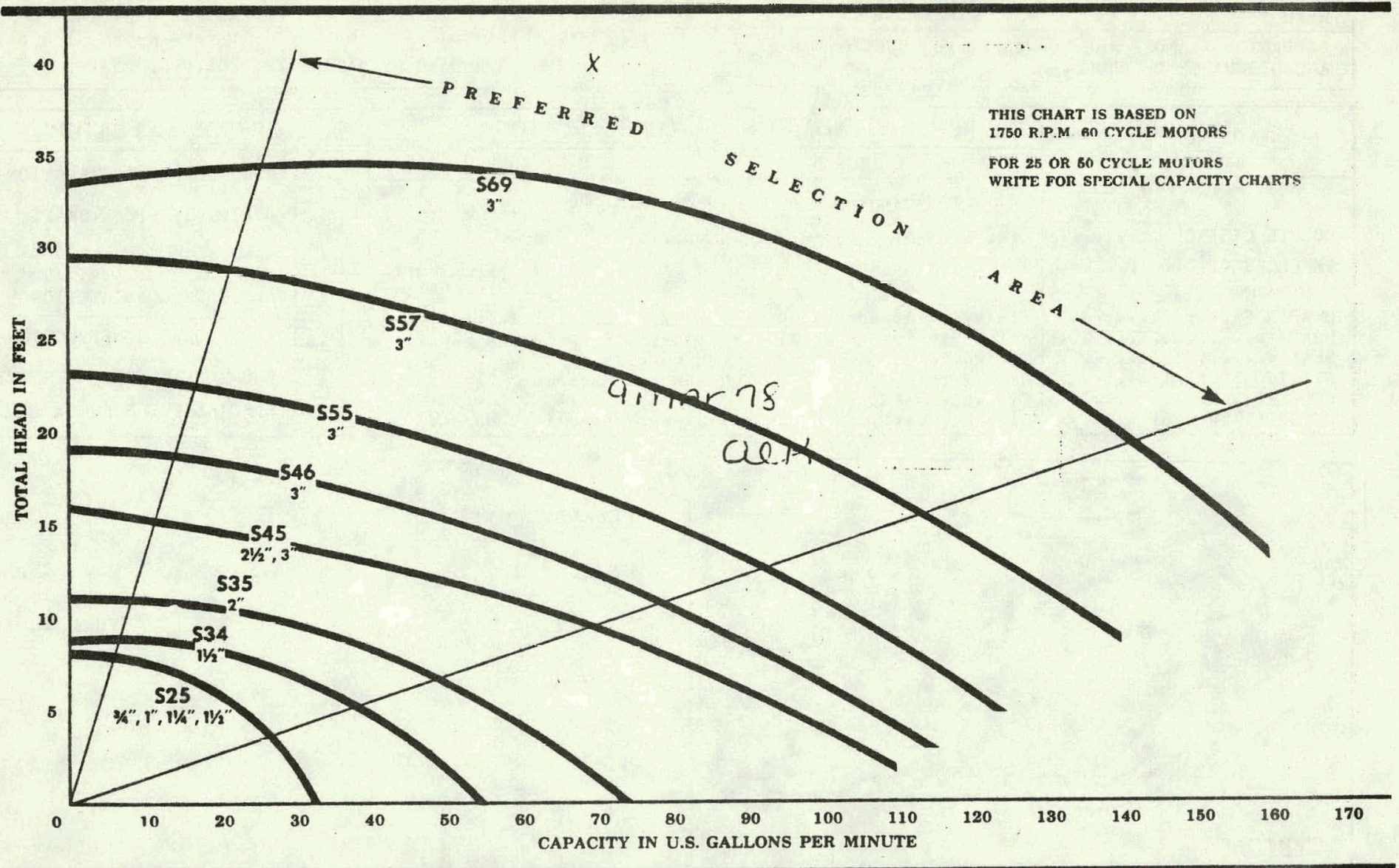

*

Dimensions shown are for reference only. For exact installation data, write Marketing

FORM. NO. $6166-$ D

Division for certified dimensions. 


\section{ARMSTRONG STANDARD CIRCULATORS SUBMITTAL DATA}

MODELS: S25-S34-S35-S45-S46-S55-S57-S69 60 CYCLE - 1750 RPM

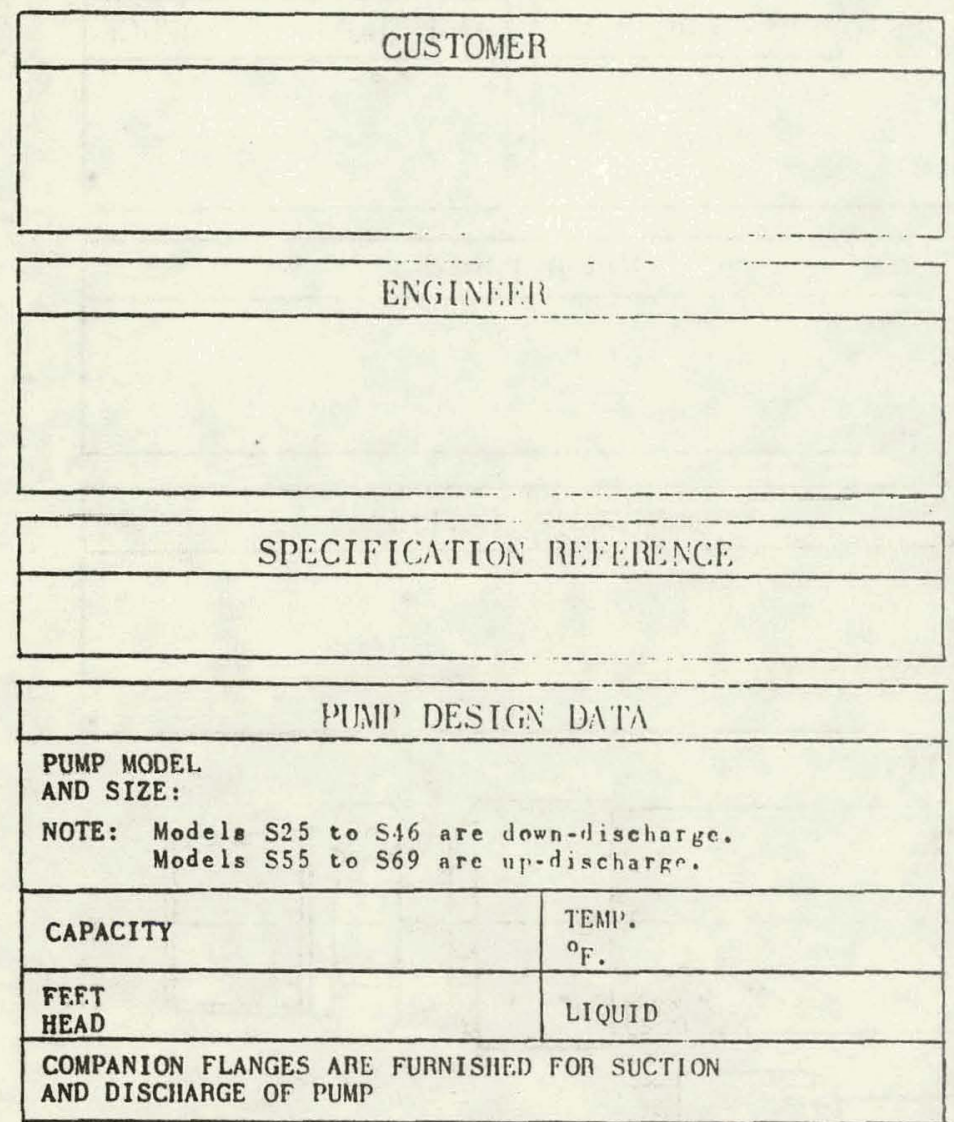

\begin{tabular}{|l|l|}
\hline CUSTOMER'S ORDER NO. & ARMSTRONG REFERENCE \\
\hline & \\
\hline
\end{tabular}
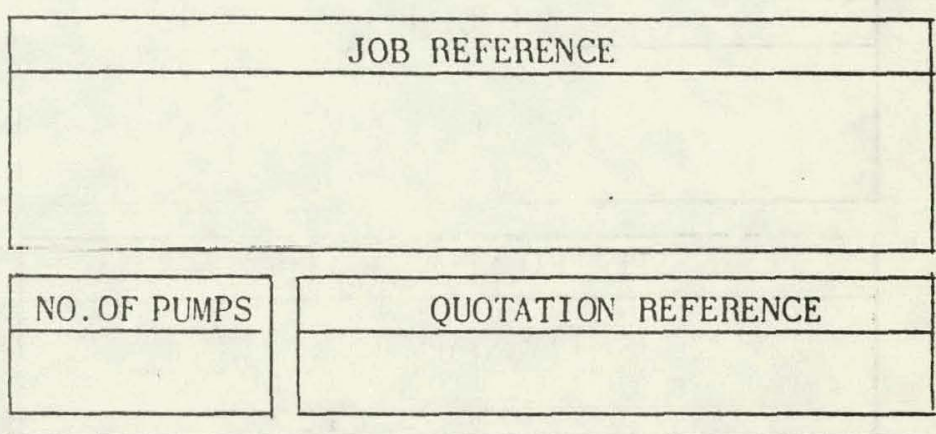

\begin{tabular}{|l|l|l|}
\hline \multicolumn{3}{|c|}{ MOTOR DESIGN DATA } \\
\hline $\begin{array}{l}\text { H.P } \\
\text { RATING }\end{array}$ & ENCLOSURE \\
\hline $\begin{array}{l}\text { SPEED } \\
\text { R.P.M. }\end{array}$ & \\
\hline VOLTS & CYCLES & PHASE \\
\hline
\end{tabular}

MAXIMUM WORKING PRESSURE: 125 psi

MAXIMUM STANDARD OPERATING TEMP: $225^{\circ} \mathrm{F}$.

\begin{tabular}{|c|c|c|}
\hline \multirow{2}{*}{ PART NAME } & \multicolumn{2}{|c|}{$\mathrm{CONSTRUCTION}$} \\
\hline & STANDARD & ALL BRONZE \\
\hline 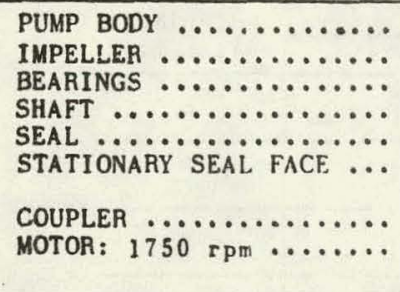 & 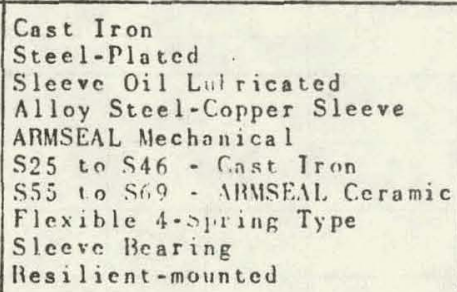 & $\begin{array}{l}\text { Bronze } \\
\text { Brass-Stamped } \\
\text { Slecve Oil Lubricated } \\
\text { Alloy Steel-Copper Sleeve } \\
\text { ARMSEAL Mechanical } \\
\text { ARMSEAL Ceramic } \\
\text { Flexible } 4 \text {-Spring Type } \\
\text { Sleeve Bearing } \\
\text { Resilient-mounted }\end{array}$ \\
\hline
\end{tabular}

\begin{tabular}{|l|}
\hline OPTIONAL FEATURES \\
\hline \\
$\square$ SPECIAL ELECTRICS \\
$\square$ SIGH TEMP. CONSTRUCTION \\
$\square$ SPECIAL MOTOR ENCLOSURE \\
$\square$ ALL IRON CONSTRUCTION \\
$\square$ BRONZE FITTED CONSTR. \\
$\square$ STAINLESS STEEL SHAFT \\
\hline
\end{tabular}

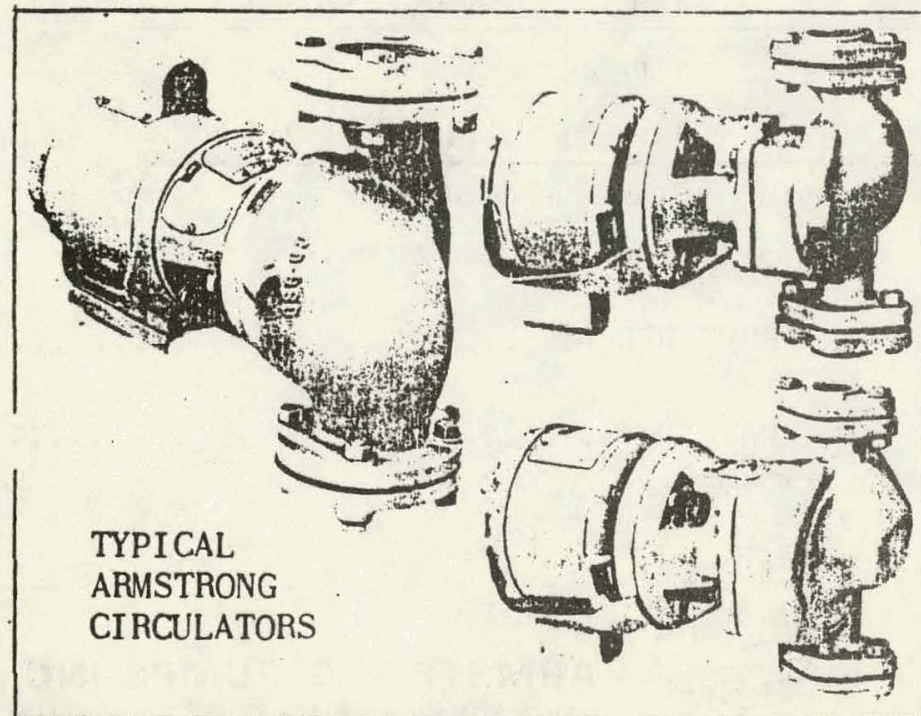

PREPARED BY:.

DATE 


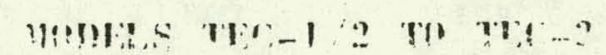
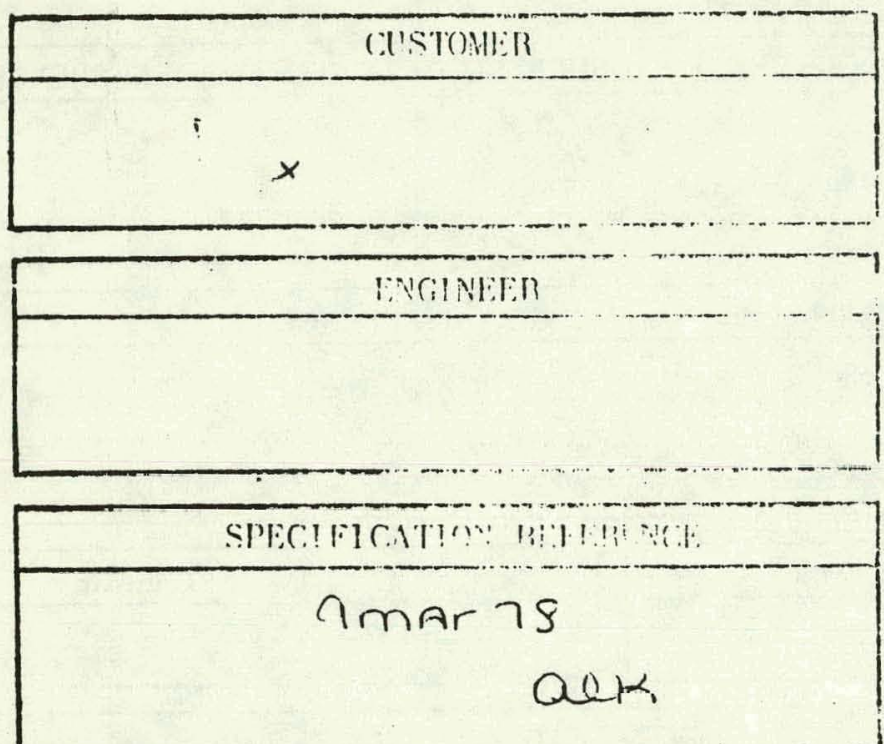

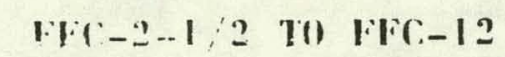

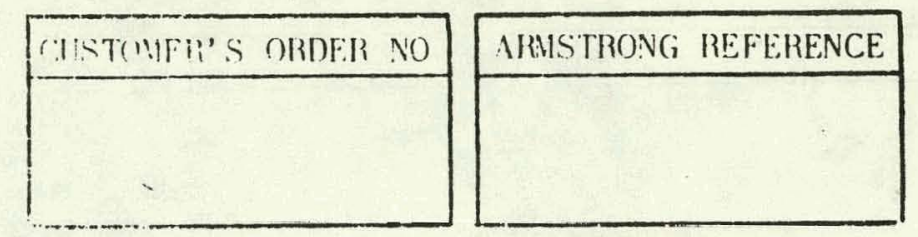

JOB REFFRFNCE:

(IIOTATON REFEHENCE

\section{BI!?!!, TEC:}
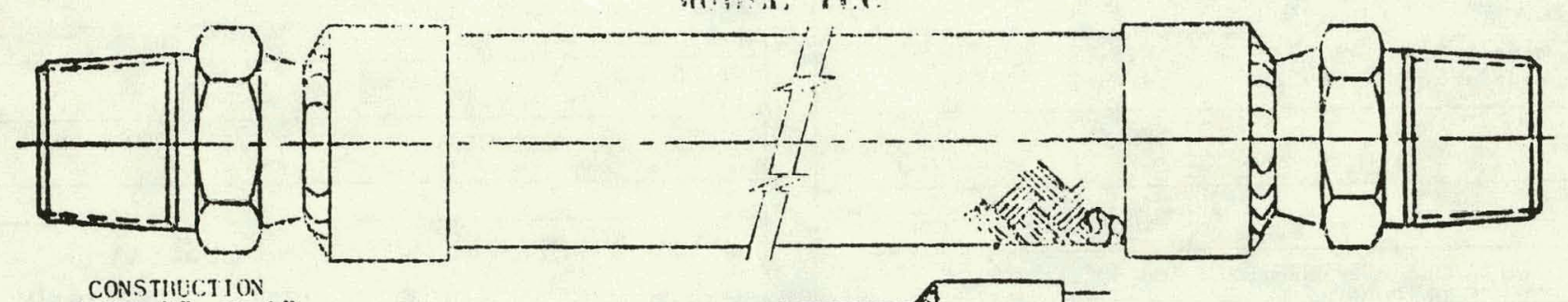

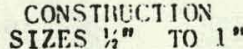

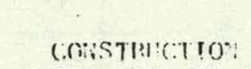
SI T.F.\& I :

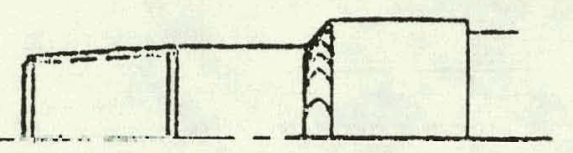

\begin{tabular}{|c|c|c|c|c|c|c|c|}
\hline \multirow{2}{*}{$\begin{array}{l}\text { (UN ITT } \\
\text { LEOH THER }\end{array}$} & \multirow[t]{2}{*}{ 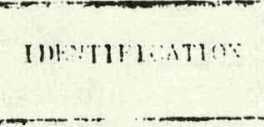 } & \multirow{2}{*}{$\begin{array}{r}\text { Mon. } \\
\text { No. }\end{array}$} & \multirow{2}{*}{ 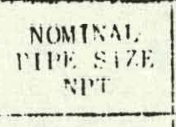 } & \multirow{2}{*}{ 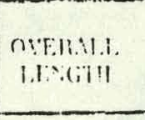 } & \multicolumn{2}{|c|}{$\begin{array}{c}\text { MA: IMIMM MOHF ING, PRFSSSIRE } \\
\text { PSIG }\end{array}$} & \multirow{2}{*}{$\begin{array}{l}\text { APPROX, } \\
\text { SHIPPING } \\
\text { HT. L.BS. } \\
\end{array}$} \\
\hline & & & & & AT $70^{\circ 1} \mathrm{~F}$ & AT $250^{6} \mathrm{~F}$ & \\
\hline & & Tl: $1 / 2$ & $1:$ & $6-1=2$ & $10+10$ & 950 & $3 / 8$ \\
\hline & & $11: 2: 1$ & 31 & $\because$ & $(6)(1)$ & 550 & $1: 2$ \\
\hline & & TES 1 & 1 & 8 & 580 & 530 & $3: 4$ \\
\hline \multirow[t]{3}{*}{24} & & Wir $1-1 / 1$ & $1-1 / 1$ & $8-1: 2$ & 150 & 410 & 1 \\
\hline & & $T ! i-1 \because$ & $1 .-1 \cdot 2$ & $n$ & .1111 & 375 & $1-1 / 1$ \\
\hline & & $\because 1: \quad$ & $\therefore$ & $111 \ldots 1 \ldots$ & $1: 11$ & 1.10 & $2-1 / 8$ \\
\hline
\end{tabular}

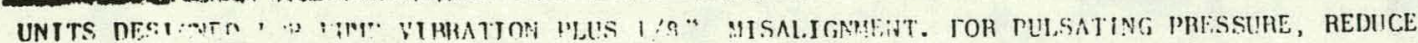

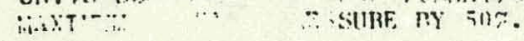

FEA IURES:

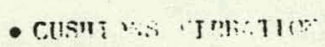

- MININizes stan " ?

PIPF. Aith bin! Jinin!

- ReDucis motse.

- saves spaci.

- LINGEK ST:I: 1 . . $1.1 \%$

FORM NO. 65.41

lit tho in $(x \| 1)$, $\because 1$ IFTI : I :

- 71, a

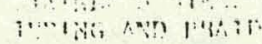

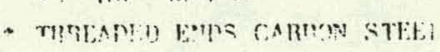

PREPARED BY

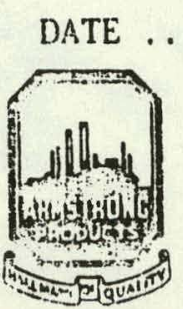

ARMSTRONG PUMPS INC. NORTH TONAWANDA $N V$ 


\section{ARMISTRONG FLEXIBİE PIMP CONNECTORS}

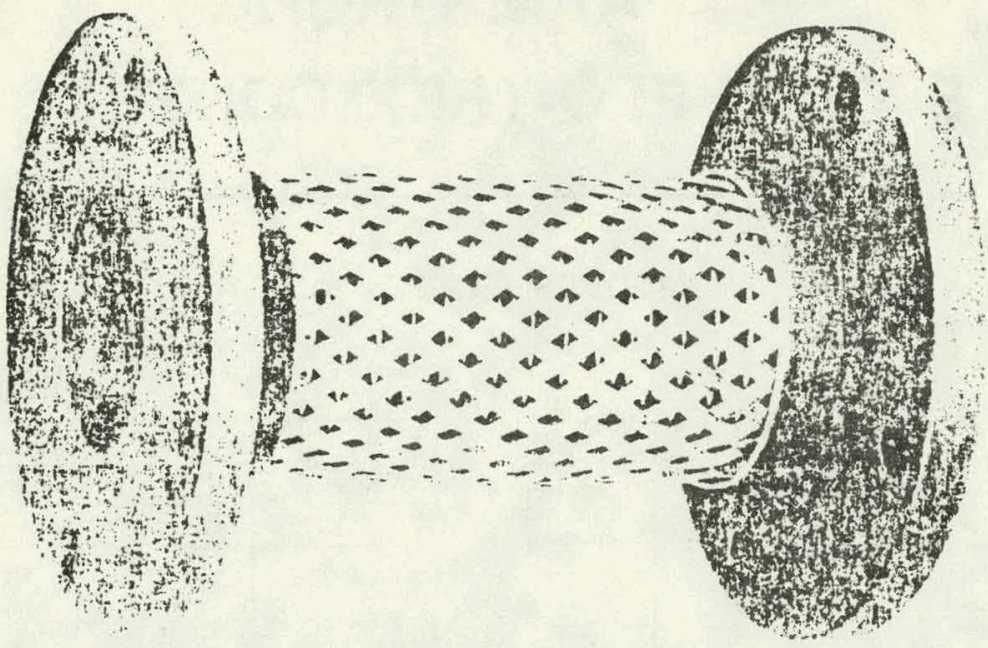

MODEL FFC

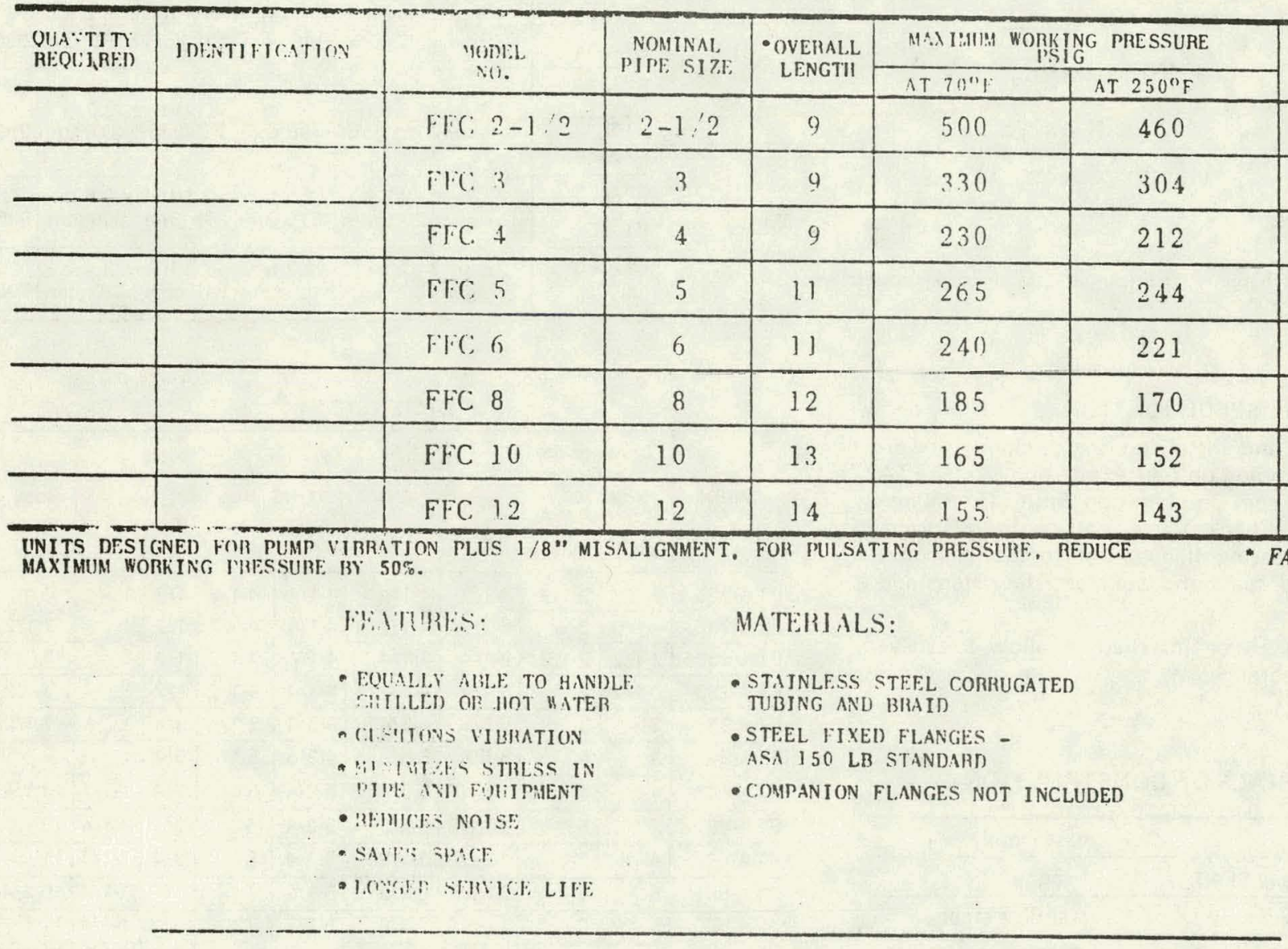

\section{GENERAL}

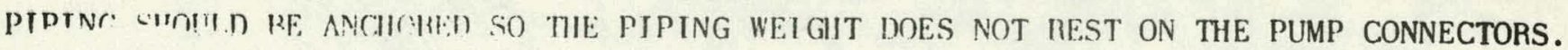

\section{SPECIFICAT] ON}

FURNISH AND INSTALL, WHITEF, SHOWN ON PLANS, ARMSTRONG FLEXIBLE PUMP CONNECTORS WITH STAINLESS STEEL BRA]DIII HELNFORCING AND FIXED ENDS. 


\section{ARMSTRONG \\ ANGLE TYPE FLO-TREX CONTROL VALVES}

MODELS FTA-225 to FTA-68

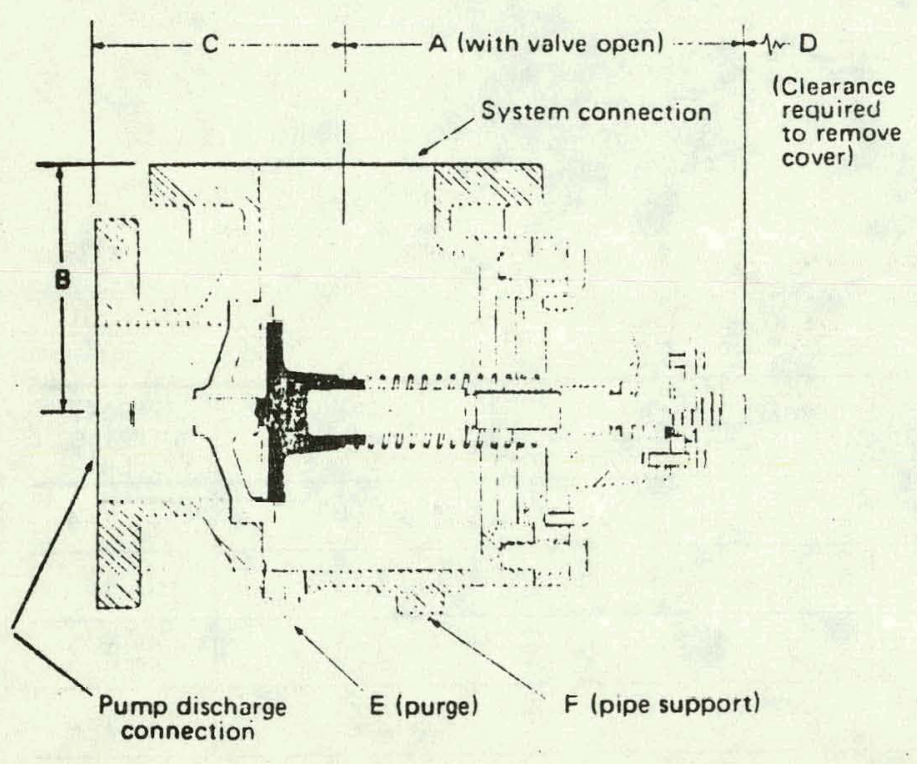

\begin{tabular}{|c|c|c|c|c|c|c|}
\hline \multirow{4}{*}{ Model } & \multicolumn{5}{|c|}{ PERFORMANCE } & \\
\hline & \multicolumn{6}{|c|}{ Pressure Drop in Feet of Water } \\
\hline & \multicolumn{6}{|c|}{ Line Velocity in Feet per Second } \\
\hline & $3 \mathrm{ft} / \mathrm{sec}$ & $4 \mathrm{ft} / \mathrm{sec}$ & $5 \mathrm{ft} / \mathrm{sec}$ & To it/sec. & $8 \mathrm{f} / \mathrm{sec}$. & $110 \mathrm{ft} / \mathrm{sec}$. \\
\hline \multicolumn{7}{|l|}{ FTA-225 } \\
\hline FTA-23 & \multirow{3}{*}{$\begin{array}{c}2.4 \mathrm{II} \\
4 \mathrm{I} \text { 'II) }\end{array}$} & \multirow{3}{*}{$\begin{array}{c}2.6 \mathrm{It} \\
\mathrm{UU}(\mathrm{II}) \mathrm{m}\end{array}$} & \multirow{3}{*}{$\begin{array}{c}2.911 \\
15 ! 11 \% 11\end{array}$} & \multirow{3}{*}{ 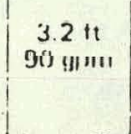 } & \multirow{3}{*}{$\begin{array}{c}4.3 \mathrm{ft} \\
120 \mathrm{yim}\end{array}$} & \multirow{3}{*}{$\begin{array}{c}C .5 \mathrm{f} \\
1 \mathrm{G}()_{4}\end{array}$} \\
\hline FTA.2525 & & & & & & \\
\hline FTA-253 & & & & & & \\
\hline FTA.33 & \multirow{2}{*}{$\begin{array}{c}2.3 \mathrm{ft} \\
70 \mathrm{gpm}\end{array}$} & \multirow{2}{*}{$\begin{array}{r}2.5 \mathrm{it} \\
92 \mathrm{gpm}\end{array}$} & \multirow{2}{*}{$\begin{array}{c}2.7 \mathrm{ft} \\
115 \mathrm{gpm}\end{array}$} & \multirow{2}{*}{$\left|\begin{array}{c}2.8 \mathrm{ft} \\
138 \mathrm{gpm}\end{array}\right|$} & \multirow{2}{*}{$\begin{array}{c}3.8 \mathrm{ft} \\
185 \mathrm{gpm}\end{array}$} & \multirow{2}{*}{$\begin{array}{c}5.7 \mathrm{fr}^{\circ} \\
230 \mathrm{gpm}\end{array}$} \\
\hline FTA-34 & & & & & & \\
\hline FTA-AS & \multirow{3}{*}{$\begin{array}{c}2.2 \mathrm{ft} \\
118 \text { ymm }\end{array}$} & \multirow{3}{*}{$\begin{array}{c}2.3 \mathrm{ft} \\
155 \mathrm{ysm}\end{array}$} & \multirow{3}{*}{$\begin{array}{c}2.5 \mathrm{ft} \\
108 \mathrm{gpm}\end{array}$} & \multirow{3}{*}{$\mid \begin{array}{c}2.7 \mathrm{ft} \\
2.38 \mathrm{gpm}\end{array}$} & \multirow{3}{*}{$\begin{array}{c}3.7 \mathrm{ft} \\
315 \mathrm{gpm}\end{array}$} & \multirow{3}{*}{$\begin{array}{c}5.5 \mathrm{hr} \\
396 \mathrm{grm}\end{array}$} \\
\hline FTA-45 & & & & & & \\
\hline FTA-46 & & & & & & \\
\hline FTA-55 & \multirow{2}{*}{$\begin{array}{c}2.1 \mathrm{ft} \\
187 \mathrm{gpm}\end{array}$} & \multirow{2}{*}{$\begin{array}{c}2.2 \mathrm{tt} \\
250 \mathrm{gpm}\end{array}$} & \multirow{2}{*}{$\begin{array}{c}2.3 \mathrm{ft} \\
315 \mathrm{gpn}\end{array}$} & \multirow{2}{*}{$\begin{array}{c}2.6 \mathrm{ft} \\
375 \mathrm{gpm}\end{array}$} & \multirow{2}{*}{$\begin{array}{c}3.5 \mathrm{ft} \\
500 \mathrm{gpm}\end{array}$} & \multirow{2}{*}{$\begin{array}{c}5.3 \mathrm{ft} \\
625 \mathrm{gpm}\end{array}$} \\
\hline FTA.56 & & & & & & \\
\hline FTA.66 & \multirow{2}{*}{$\begin{array}{c}2.0 \mathrm{ft} \\
270 \mathrm{gpm}\end{array}$} & \multirow{2}{*}{$\begin{array}{c}2.1 \mathrm{ft} \\
360 \mathrm{gpm}\end{array}$} & \multirow{2}{*}{$\begin{array}{c}2.2 \mathrm{ft} \\
450 \mathrm{gpm}\end{array}$} & \multirow{2}{*}{$\begin{array}{c}2.3 \mathrm{ft} \\
540 \mathrm{gpm}\end{array}$} & \multirow{2}{*}{$\begin{array}{c}3.4 \mathrm{ft} \\
720 \mathrm{gpm}\end{array}$} & \multirow{2}{*}{$\begin{array}{c}5.1 \mathrm{ft} \\
900 \mathrm{gpm}\end{array}$} \\
\hline FTA.68 & & & & & & \\
\hline
\end{tabular}

\section{TYPICAL SPECIFICATION}

Furnish and install on the discharge line of each pumping unit an Armstrong Model FTA Angle Type Combination Shut;off, Balancing and Check Valve, with positive spring. loaded seating, flanged cast iron body, bronze disc and seat, and stainless steel stem and spring.

Valve is to be installed to allow for valve stem clearance.

\section{XMATERIALS OF CONSTRUCTION}

\begin{tabular}{ll}
\hline BODY & cast iron \\
\hline DISC and SEAT & bronze \\
\hline STEM & stainless steel \\
\hline SPRING & stainless stecl \\
\hline PACKING & graphited asbestos \\
\hline
\end{tabular}

9 mar 78

acr
DIMENSIONS *

\begin{tabular}{|c|c|c|c|c|c|c|c|c|c|c|}
\hline \multirow[t]{2}{*}{ Model } & \multicolumn{2}{|c|}{$\begin{array}{c}\text { Connection } \\
\text { Sizes } \\
\text { Flanged } \\
125 \text { Ib A.S.A. }\end{array}$} & \multicolumn{6}{|c|}{ DIMENSIONS (inches) } & \multirow[t]{2}{*}{$\begin{array}{l}\text { Max. } \\
\text { Body } \\
\text { Din. }\end{array}$} & \multirow[t]{2}{*}{$w_{1}$} \\
\hline & Inlet & Outlet & A & B & C & v & {[} & $F$ & & \\
\hline FTA-225 & 2 & $2 \cdot 1 / 2$ & $8-1 / 4$ & $4-1 / 4$ & $4-1 / 4$ & $4-1 / 2$ & $1 / 2$ & 1 & 7 & 38 \\
\hline FTA-23 & 2 & 3 & $8-1 / 4$ & $4 \cdot 11 / 16$ & $41 / 4$ & $4-1 / 2$ & $1 / 2$ & 1 & $7.1 / 2$ & 42 \\
\hline FTA-2525 & $2 \cdot 1 / 2^{\circ}$ & $2 \cdot 1 / 2$ & $8-1 / 4$ & $4 \cdot 1 / 4$ & $4-1 / 4$ & $4-1 / 2$ & $1 / 2$ & 1 & 7 & 43 \\
\hline FTA.253 & $2 \cdot 1 / 2$ & 3 & $8.1 / 4$ & $4-11 / 16$ & $4 \cdot 1 / 4$ & $4.1 / 2$ & $1 / 2$ & 1 & $7.1 / 2$ & 50 \\
\hline FTA-33 & 3 & 3 & $8.5 / 8$ & $4 \cdot 3 / 4$ & $4-3 / 4$ & $5-1 / 4$ & $3 / 4$ & 1 & $7-1 / 2$ & 56 \\
\hline FTA.34 & 3 & 4 & $8 \cdot 5 / 8$ & $5-5 / 8$ & $4-3 / 4$ & $5-1 / 4$ & $3 / 4$ & 1 & 9 & $65^{3}$ \\
\hline FTA.44 & 4 & 4 & $10-1 / 8$ & $5 \cdot 3 / 4$ & $5 \cdot 3 / 4$ & 6 & $3 / 4$ & $1.1 / 4$ & $9.1 / 4$ & 98 \\
\hline FTA-45 & 4 & 5 & $10-1 / 8$ & $6.5 / 8$ & $5-3 / 4$ & 6 & $3 / 4$ & $1.1 / 4$ & 10 & 102 \\
\hline FTA-46 & 4 & 6 & $10-1 / 8$ & $7 \cdot 1 / 2$ & $5-3 / 4$ & 6 & $3 / 4$ & $1.1 / 4$ & 11 & $107^{\circ}$ \\
\hline FTA.55 & 5 & 5 & $11-5 / 8$ & $6 \cdot 1 / 2$ & $6-1 / 2$ & 7 & 1 & $1.1 / 4$ & $10-3 / 4$ & 138 \\
\hline FTA-56 & 5 & 6 & $11.5 / 8$ & $7 \cdot 3 / 8$ & $6-1 / 2$ & 7 & 1 & $1.1 / 4$ & 11 & 142 \\
\hline FTA-66 & 6 & 6 & 13 & 7 & 7 & 8 & 1 & 2 & $12-3 / 4$ & $148^{\circ}$ \\
\hline FTA-68 & 6 & 8 & 13 & $8-3 / 4$ & 7 & 8 & 1 & 2 & {$[13.1 / 2$} & $153^{-1}$ \\
\hline
\end{tabular}

* Consult factory for certified dimensions

\section{DESIGN INFORMATION} Maximum working pressure . ... 175 psig Maximum working temperature. . $300^{\circ} \mathrm{F}$ 


\section{ARMSTRONG SÜCTION GUIDE SUBMITTAL DATA}

MODELS: SG-252 TO SG-1010

\begin{tabular}{|c|}
\hline CUSTOMER \\
\hline \\
\end{tabular}

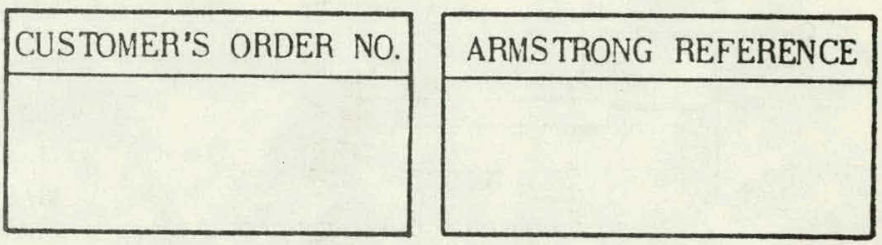

ENGINEER

SPECIFICATION REFERENCE

\section{QUANTITIES REQUIRED}

MAXIMUM WORKING PRE.SSURE 175 PSIG - MAXIMUM WORK ING TEMPERATURE $300^{\circ} \mathrm{F}$.

\begin{tabular}{l|l|l|l|l|}
\hline \multirow{2}{*}{ MODEL NUMBER } & \multicolumn{2}{|c|}{ CONNECTION SIZES 125 LB A.S.A. FLANGED } & \multirow{2}{*}{ QUANTITY REQUIRED } & \multirow{2}{*}{ IDENTIFICATION } \\
\cline { 2 - 5 } & INLET FRON SYSTEM & OUTLET TO PUMP & & \\
\hline & & & & \\
\hline & & & & \\
\hline
\end{tabular}

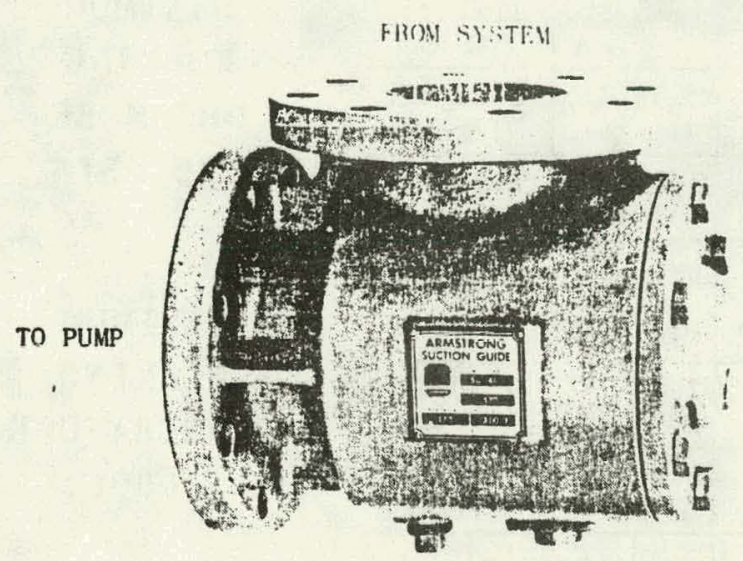

TYPE SG SUCTION GUIDE

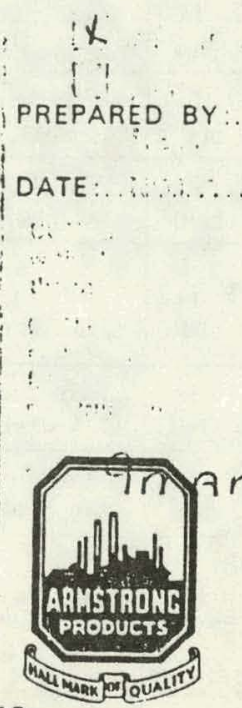

B5 - 19

\section{QUOTATION REFERENCE}

JOB REFERENCE 


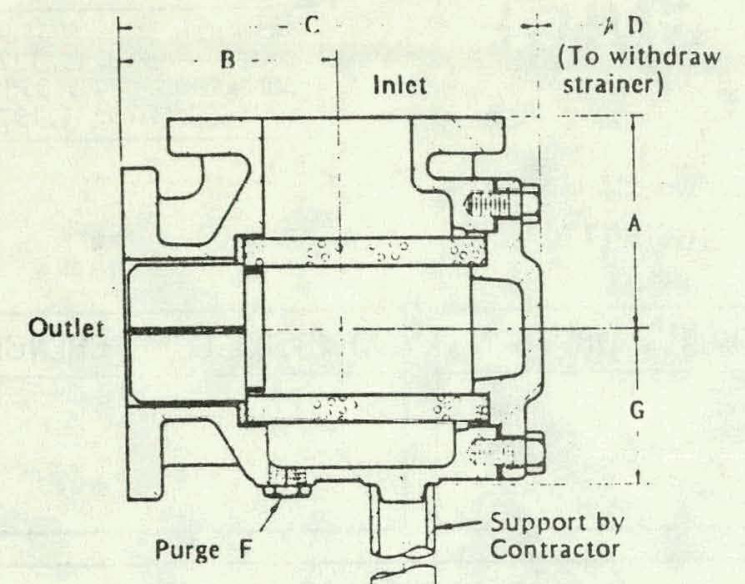

- ARMSTRONG SUCTION GUIDE

FOR USE ON SUCTION CONNECTION OF CENTIIIVUGAL PUMP ... TO SAVE SPACE, SIMPLIFY PIPING AND PROVIDE STEADY FLOW CONDITION AT PUMP INLET.

\section{MATERIAL}

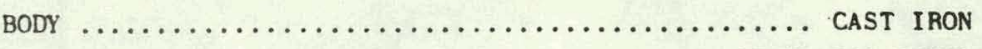

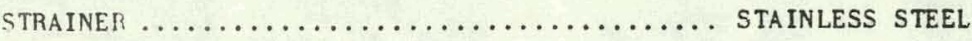

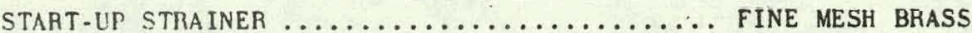

(Remove and dispose of start-up strainer after 1 month operation) GUIDE VANES $\ldots \ldots \ldots \ldots \ldots \ldots \ldots \ldots \ldots \ldots \ldots \ldots \ldots \ldots \ldots \ldots \ldots \ldots$
COMPANION FLANGE

NOT INCIMIIEFE

\section{DIMENSIONS}

\begin{tabular}{|c|c|c|c|c|c|c|c|c|c|c|c|c|c|}
\hline \multirow{2}{*}{ MODEL NO. } & \multicolumn{2}{|c|}{ 125" A.S.A. } & \multirow{2}{*}{$\begin{array}{c}\text { STRA INER } \\
\text { OPE.NINGS } \\
\text { DIA. }\end{array}$} & \multirow{2}{*}{\begin{tabular}{|c|} 
STRA INEH \\
FREE ARE. A \\
(SO. IN) \\
\end{tabular}} & \multicolumn{8}{|c|}{ DIMENSIONS (INCHES) } & \multirow{2}{*}{ 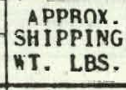 } \\
\hline & INLET & OUTLET & & & $A$ & $\mathrm{~B}$ & C & $\mathrm{D}$ & जIDTH E & $\mathrm{F}$ & G & $\mathrm{H}$ & \\
\hline $5 G-252$ & $21 \div 2$ & 2 & $18 \prime \prime$ & $3 i$ & $4-14$ & $4-1.4$ & $8-1 ; 2$ & $4-3: 4$ & $i$ & $1: 2$ & $3-1 / 8$ & 1 & 36 \\
\hline $5 G-32$ & 3 & 2 & 18 & 37 & $4-1116$ & $4-1,4$ & $8-1,2$ & $4-3: 4$ & $7-1: 2$ & $1: 2$ & $3-1 ; 8$ & 1 & 37 \\
\hline $5 G-2525$ & 212 & 212 & 18 & 37 & $4-14$ & $4-1: 4$ & $8-1: 2$ & $4-3 \quad 4$ & 7 & $1 / 2$ & $3-1 ; 8$ & 1 & 40 \\
\hline $\mathrm{SG}-325$ & 3 & $2 !:$ & 18 & 37 & $4-1116$ & $4-1,4$ & $8-1: 2$ & $4 \cdot 3 / 4$ & $7-1,2$ & $1 / 2$ & $3-1 / 8$ & 1 & 41 \\
\hline SG -33 & 3 & 3 & 1,8 & 50 & $4-3: 4$ & $4-3 / 4$ & $9-1 / 2$ & $5-1,2$ & $7-1 / 2$ & $3 / 4$ & $3-5 / 8$ & 1 & 53 \\
\hline $\mathrm{SG}-43$ & 4 & 3 & 18 & 50 & 5.58 & $4-3: 4$ & $9-1 / 2$ & $5-1: 2$ & 9 & $3 / 4$ & $3-5 ; 8$ & 1 & 55 \\
\hline SG-44 & 4 & 4 & $1: 8$ & 82 & $5-3: 4$ & $5-3 ; 4$ & $11-1 ' 2$ & 7 & 9 & $3 / 4$ & $4-1: 4$ & $11 / 4$ & 75 \\
\hline SG- 54 & 5 & 4 & $1: 8$ & 82 & $6-5 ' 8$ & $5-3 ; 4$ & $11-1,2$ & 7 & 10 & $3 / 4$ & $4-1 ; 4$ & $11 / 4$ & 81 \\
\hline SG-64 & 6 & 4 & $1: 8$ & 82 & $7-1 ' 2$ & $5 \cdot 34$ & $11-1: 2$ & 7 & 11 & $3 / 4$ & $4-1 / 4$ & $1 \frac{1}{4}$ & 86 \\
\hline SG - 55 & 5 & 5 & 18 & 104 & $6-1 / 2$ & $6-1: 2$ & $12-34$ & 8 & 10 & 1 & $4-7 ! 8$ & $11 / 4$ & 95 \\
\hline SG-65 & 6 & 5 & $1: 8$ & 104 & $7-3 ; 8$ & $6-1 / 2$ & $12-3: 4$ & 8 & 11 & 1 & $4-7 / 8$ & $11 / 4$ & 106 \\
\hline SG-66 & 6 & 6 & 18 & 153 & 7 & 7 & $14.1{ }^{\prime} 4$ & $9-1 / 4$ & $11-1 / 4$ & 1 & $5-3 / 4$ & 2 & 146 \\
\hline SG. 86 & 8 & 6 & 18 & 153 & $8-34$ & 7 & $14-1 / 4$ & $9-1: 4$ & $13-1,2$ & 1 & $5-3 / 4$ & 2 & 151 \\
\hline $\mathrm{SG}-88$ & 8 & 8 & $1: 8$ & 275 & $9-14$ & $9.1: 4$ & 18 & $12-1 ; 4$ & $13-3 / 4$ & 1 & 7 & 2. & 240 \\
\hline SG- 108 & 10 & 8 & 1.8 & 275 & 11 & 9.14 & 18 & $12-1: 4$ & 16 & 1 & $i$ & 2 & 265 \\
\hline SG- 1010 & 10 & 10 & $1: 8$ & 420 & $11-14$ & $11 \cdot 1 \cdot 4$ & $22-1 / 2$ & 15 & 16 & 1 & $8-1 / 8$ & 2 & 360 \\
\hline
\end{tabular}

\section{PERFORMANCE}

\begin{tabular}{|c|c|c|c|c|c|c|c|}
\hline \multirow{3}{*}{ MODEL NO. } & \multicolumn{7}{|c|}{ PRESSURE IDROP IN FEET OF WATER } \\
\hline & \multicolumn{7}{|c|}{ VELOCITY IN FT. ISEC. } \\
\hline & 3 FT. SEC. & 4 FT. SEC. & 5 FT. SEC. & i, FT. SEC & $8 \mathrm{FT} . \mathrm{SEC}$. & 10 FT. SEC. & 12 FT. ISEC. \\
\hline $\begin{array}{l}\text { SG-252 } \\
\text { SG-32 } \\
\end{array}$ & $\begin{array}{l}11 \mathrm{ft} \\
(32 \mathrm{GPM})\end{array}$ & $\begin{array}{r}20 \mathrm{ft} \\
(43 \mathrm{GPM}) \\
\end{array}$ & $\begin{array}{l}32 \mathrm{fe} \\
(54 \mathrm{GPM})\end{array}$ & $\begin{array}{c}.3 \mathrm{ft} \\
(64 \mathrm{GPM}) \\
\end{array}$ & $\begin{array}{l}80 \mathrm{ft} \\
(0 \mathrm{~s} \mathrm{GPM})\end{array}$ & $\begin{array}{r}1.28 \mathrm{ft} \\
(108 \mathrm{GPM})\end{array}$ & $\begin{array}{l}1.72 \mathrm{ft} . \\
(128 \mathrm{GPM})\end{array}$ \\
\hline $\begin{array}{l}\text { SG- } 2525 \\
\text { SG- } 325 \\
\end{array}$ & $\begin{array}{l}18 \mathrm{fL} \\
(45 \mathrm{GPM})\end{array}$ & \begin{tabular}{|lr}
32 & $\mathrm{ft}$ \\
$(60$ & Gi'M) \\
\end{tabular} & $\begin{array}{c}50 \mathrm{ft} \\
(: 5 \mathrm{GP} \mathrm{M}) \\
\end{array}$ & $\begin{array}{c}.72 \mathrm{ft} \\
(90 \mathrm{GMM})\end{array}$ & $\begin{array}{l}1.28 \mathrm{ft} \\
(120 \mathrm{GPM})\end{array}$ & $\begin{array}{r}2.00 \mathrm{ft} \\
(150 \mathrm{GPM}) \\
\end{array}$ & $\begin{array}{l}2.88 \mathrm{ft} . \\
(\mathrm{i} 80 \mathrm{GPM})\end{array}$ \\
\hline $\begin{array}{l}\text { SG-33 } \\
\text { SG-43 } \\
\end{array}$ & $\begin{array}{l}20 \mathrm{ft} . \\
(\mathrm{i} 0 \mathrm{GPM})\end{array}$ & $\begin{array}{l}.35 \mathrm{ft} \\
(92 \mathrm{GPM}) \\
\end{array}$ & \begin{tabular}{|c|}
$55 \mathrm{ft}$ \\
$(115 \mathrm{GPM})$ \\
\end{tabular} & $\begin{array}{r}.80 \mathrm{ft} \\
(138 \mathrm{GPM}) \\
\end{array}$ & $\begin{array}{c}1.42 \mathrm{ft} . \\
(185 \mathrm{GPM})\end{array}$ & $\begin{array}{r}2.20 \mathrm{ft} \\
(230 \mathrm{GPM})\end{array}$ & $\begin{array}{l}3.15 \mathrm{ft} . \\
(276 \mathrm{GPM})\end{array}$ \\
\hline $\begin{array}{l}S G-44 \\
S G-54 \\
S G=64 \\
\end{array}$ & $\begin{array}{c}17 \mathrm{ft} \\
(118 \mathrm{GPM})\end{array}$ & $\begin{array}{l}.29 \mathrm{ft} \\
(155 \mathrm{GPM})\end{array}$ & $\begin{array}{r}.47 \mathrm{ft} \\
(198 \mathrm{GPM})\end{array}$ & $\begin{array}{l}.68 \mathrm{ft} \\
(238 \mathrm{GPN})\end{array}$ & $\begin{array}{l}1.20 \mathrm{ft} \\
(315 \mathrm{GPM})\end{array}$ & $\begin{array}{c}1.90 \mathrm{ft} \\
(396 \mathrm{GPM})\end{array}$ & $\begin{array}{l}2.75 \mathrm{ft} . \\
(475 \mathrm{GPM})\end{array}$ \\
\hline $\begin{array}{l}\text { SG- } 55 \\
\text { SG-65 } \\
\end{array}$ & $\begin{array}{r}23 \mathrm{ft} \\
(18: \mathrm{GPM}) \\
\end{array}$ & $\begin{array}{rr}41 & \mathrm{ft} \\
(250 & \mathrm{GPM}) \\
\end{array}$ & $\begin{array}{r}.67 \mathrm{ft} . \\
(315 \mathrm{GPM})\end{array}$ & $\begin{array}{r}.95 \mathrm{ft} \\
(3 i 5 \mathrm{GPM})\end{array}$ & $\begin{array}{l}1.70 \\
(500 \mathrm{ft}\end{array}$ & $\begin{array}{l}2.65 \mathrm{ft} . \\
(625 \mathrm{GPM})\end{array}$ & $\begin{array}{l}3.80 \mathrm{ft} . \\
(750 \mathrm{GPM})\end{array}$ \\
\hline $\begin{array}{l}\text { SG- } 66 \\
\text { SG- } 86 \\
\end{array}$ & $\begin{array}{rr}.22 \mathrm{ft} \\
(270 \mathrm{GPM}) \\
\end{array}$ & $\begin{array}{r}.38 \mathrm{ft} \\
(360 \mathrm{GPM}) \\
\end{array}$ & $\begin{array}{c}.60 \mathrm{ft} \\
(450 \mathrm{GPM})\end{array}$ & $\begin{array}{r}.86 \mathrm{fl} \\
(540 \mathrm{GPM})\end{array}$ & $\begin{array}{r}1.54 \mathrm{ft} . \\
(720 \mathrm{GPM})\end{array}$ & $\begin{array}{c}2.40 \mathrm{ft} . \\
(900 \mathrm{GPM})\end{array}$ & $\begin{array}{l}3.45 \mathrm{ft} \\
(1080 \mathrm{GPM})\end{array}$ \\
\hline $\begin{array}{l}\text { SG }-88 \\
\text { SG- } 108 \\
\end{array}$ & $\begin{array}{rr}21 & \mathrm{ft} \\
(470 & \mathrm{GPM}\end{array}$ & $\begin{array}{r}37 \mathrm{ft} \\
(625 \mathrm{GPU}) \\
\end{array}$ & $\begin{array}{c}.58 \mathrm{ft} \\
(780 \mathrm{GPM}) \\
\end{array}$ & $\begin{array}{c}.85 \mathrm{ft} \\
(940 \mathrm{GPM})\end{array}$ & $\begin{array}{l}1.50 \\
(1250\end{array}$ & 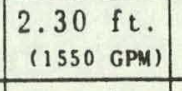 & $\begin{array}{l}3.35 \mathrm{ft} . \\
(18 \div 0 \mathrm{GPM})\end{array}$ \\
\hline SG -1010 & $\begin{array}{cc}26 & \mathrm{ft} \\
(740 & \mathrm{GPM}\end{array}$ & $\begin{array}{cc}46 & \mathrm{ft} \\
i 980 & \mathrm{GPM} \\
\end{array}$ & $\begin{array}{cc}73 & f t \\
(i 230 & \mathrm{GPM}\end{array}$ & $\begin{array}{lll}1.03 & \mathrm{ft} \\
(1480 \mathrm{GPM})\end{array}$ & \begin{tabular}{|lll}
1.85 & $\mathrm{ft}$ \\
$(1960$ & $\mathrm{GPM})$ \\
\end{tabular} & $\begin{array}{ccc}2.92 & f t \\
(2450 & \text { GPM }) \\
\end{array}$ & $\begin{array}{r}4.20 \mathrm{ft} . \\
(2940 \mathrm{GPM})\end{array}$ \\
\hline
\end{tabular}

\section{MAXIMUM \\ WORK ING \\ PRESSURE \\ 175 PSIG}

\section{MAXIMUM \\ WORKING \\ TEMPERATURE \\ $300^{\circ} \mathrm{F}$.}




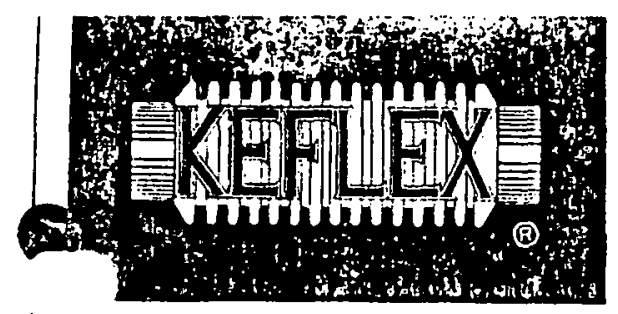

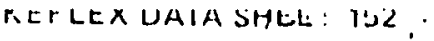 \\ DIFFERERTIAL \\ PUMP CONIECTORS}

\section{NEW PRODUCT PRE-CATALOG RELEASE}

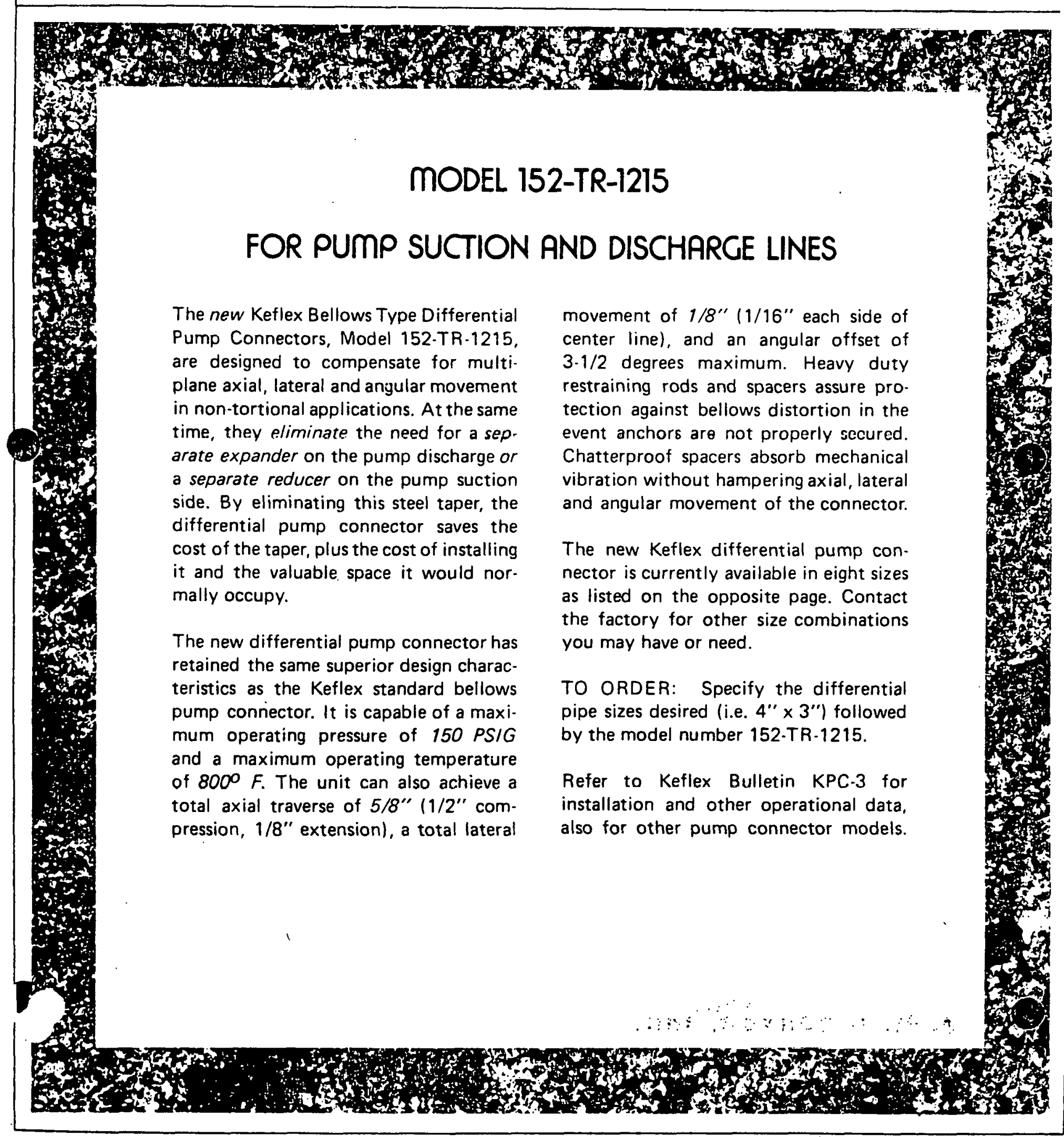


This page has been removed because of copyright material. For information on the Keflex Differential Pump Connectors, contact

Keflex, Inc., 225 S. Main Street, Bartlett, Illinois 60103. 


\section{ARMSTRONG VORTEX AIR SEPARATOR SUBMITTAL DATA}

\begin{tabular}{lrr|}
\hline FILE NO, & 5035.955 \\
DRIE: & SePt. 20,1974 \\
SUPERSEDES: & 5035.955 \\
DATE: & MaY $10,: 974$ \\
\hline
\end{tabular}

1

MODELS: VA-2 TO VA-12 LESS STIMINER

VAS- 2 TO VAS-12 H'ITH STRAINER

\begin{tabular}{|c|}
\hline CUSTONER \\
\hline \\
\hline ENGINEER \\
\hline \\
\hline \\
\hline
\end{tabular}

CISTONER'S ORDER NO.

ARMSTIRONG REFERENCE

SPECIFICATION REFERENCE

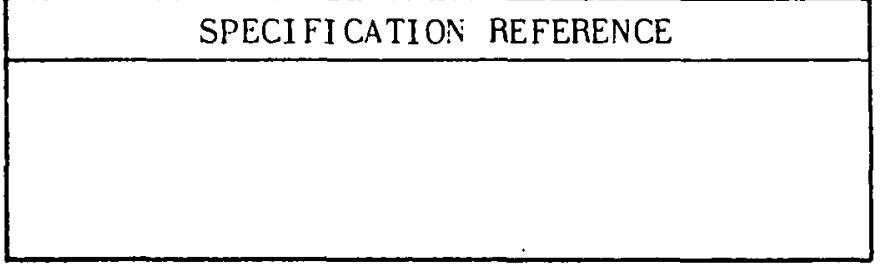

\begin{tabular}{|l|l|}
\hline MODEL NUMBER & MAXIMUM FLOW \\
\hline & USGPN \\
\hline
\end{tabular}

CONSTRUCTION OF VORTEX AIR SEPARATOR

Fabricated steel shell constructed according to ASMECode for Unfired Pressure Vesseis.

Form U.IA Manufacturer's Data Sheet signed by N.B. Inspector provided in accordance with ASME Code for the foliowing units ONLY: VA5 - VASS to VA12 - VAS12 inclusive.

Certificates are not required, and are not available, for smaller Units VA2 - VAS2 to VA4 - VAS4 inclusive.

Connections provided for:

(a) Main inlet and outlet flow .

(b) Air connections for line to compression tank

(c) Drain flushing connection at bottom to eliminate dirt and sediment

(d) With strainer () without strainer ()

U.S. PATENT NO. 3771290

\section{RENAARKS AND/OR ACCESSORIES}

DIMENSIONS: SEE REVERSE SIDE
QUOTATION REFERENCE

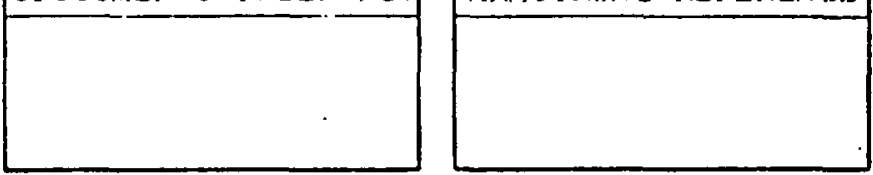

JOB REFERENCF:
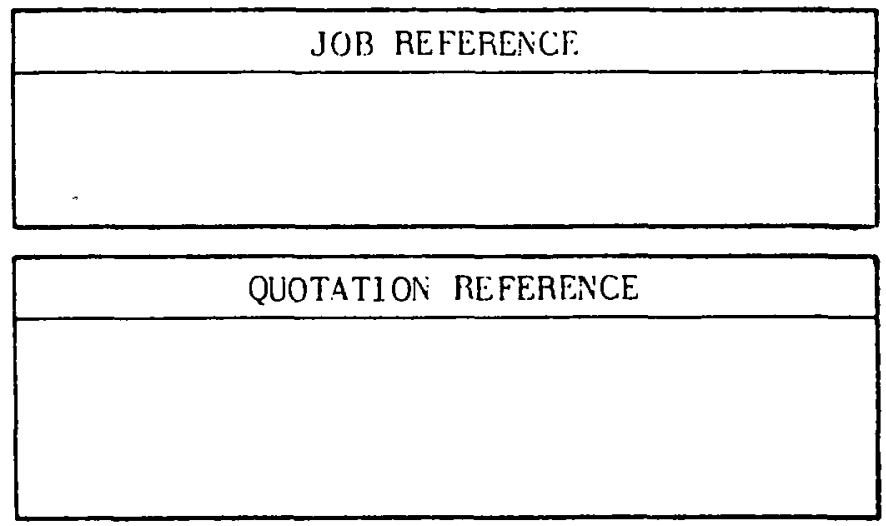

WORKING CONDITIONS

MAXIMUN WORKING PRESSURE .......... 125 psi MAXIMUM FORKING TEMPERATURE ........ $350^{\circ} \mathrm{F}$ HYDROSTATIC TEST PRESSURE .......... 200 psi

\section{SPECIFICATIONS}

VERTICALLY MOUNTED AIR SEPARATOR HAVING STEEL TANK WITH TANGENTIAL INLET AND OUT. LET FOR SEPARATION OF AIR IN HYDRONIC SYSTEM AND TO ACT AS A SEDIMENT TRAP. REMOVABLE STEEL AIR COLLECTOR TUBE.

SUITABLE FOR PIPE-LINE MOUNTING.

PREPARED BY:

DATE :

Form No. 6429.D

Litho in Canada

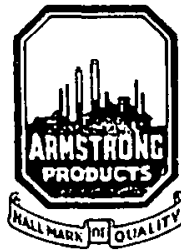

ARMSTRONG PUMPS INC. North Tonawanda, N. Y. 14120 
NOTES:

1. INLET AND OUTLET CONNECTIONS "A" ARE NPT FOR SIZES 2 " TO 3" AND 150 " A.S.A. (RF) FLANGED FOF SIZES 4 " TO 12 "

2. HOLT HOLES IN ALL FLANGES STRADDLE VERTICAL \&

MAXIMUM WORKING PRESSURE 125 PSIG

MAXIMUM WORKING TEMPERATURE $350^{\circ} \mathrm{F}$

"B" NPT AIR OUTLET
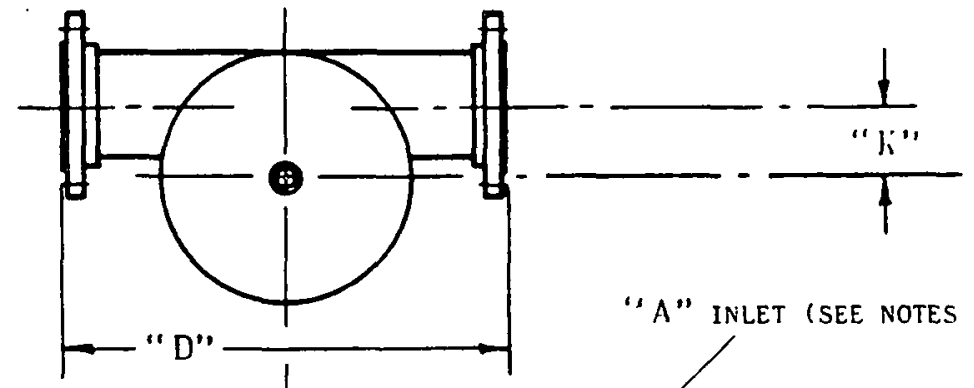

"A" INLET (SEE NOTES $1 \& 2$ ) "D"

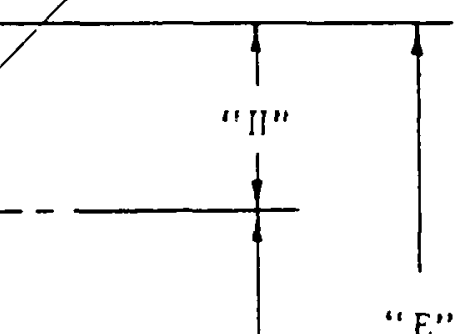

"A" OUTLET (EEe NOTES I \& 2)

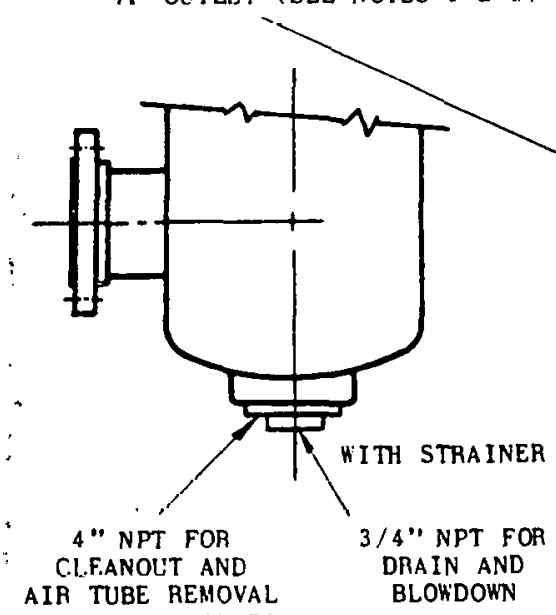

(3" NPT ON SIZES

VAS $-2 \&$ VAS - $\left.2 \frac{1}{2}\right)$

DRAIN AND BLOKDOWN
TURE
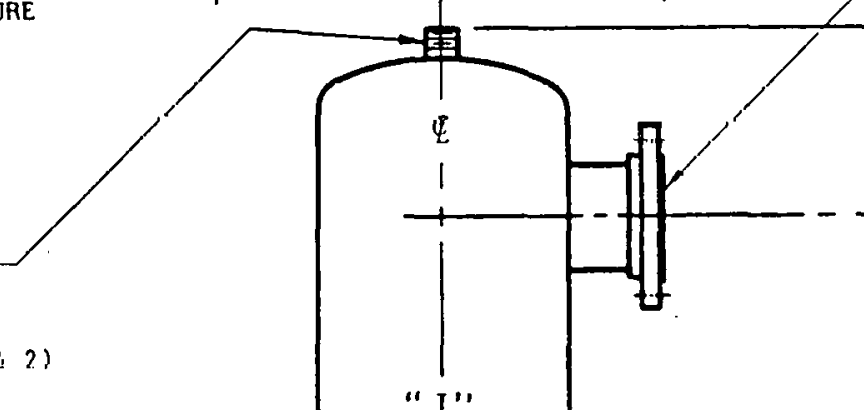

"J"

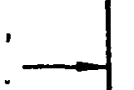

B" NPT FOR

DRAIN AND

AIR TUBE REMOVAL
"G"MINIMUM DIS.

TANCE HEUUIHED FUK AIR TUBE REMOVAL
"F" (APPROXIMATE)

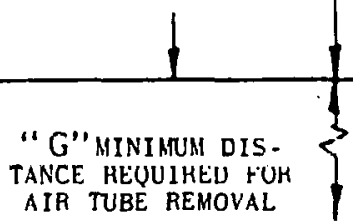

\begin{tabular}{|c|c|c|c|c|c|c|c|c|c|c|c|c|c|c|c|c|}
\hline \multicolumn{2}{|c|}{ MODEL NUMBER } & \multicolumn{12}{|c|}{ DIMENSIONS (IN INCHES) } & \multirow{4}{*}{$\begin{array}{l}\text { MAX. } \\
\text { FLOK' } \\
\text { USGPM } \\
60\end{array}$} & \multirow{2}{*}{\multicolumn{2}{|c|}{$\begin{array}{l}\text { APPROX. } \\
\text { WE I GHT LDS. }\end{array}$}} \\
\hline LESS & WI TH & & $\cdot$ & & & $E$ & $F$ & $\mathrm{E}$ & $F$ & \multirow{2}{*}{$G$} & \multirow[b]{2}{*}{$\mathrm{H}$} & \multirow{2}{*}{$\mathrm{J}$} & \multirow[b]{2}{*}{ K } & & & \\
\hline STR. & STR. & A & B & C & $\mathrm{D}$ & \multicolumn{2}{|c|}{ LESS STHAINER } & \multicolumn{2}{|c|}{ WITH STRAINEA } & & & & & & \begin{tabular}{|c|} 
LESS \\
STR. \\
\end{tabular} & $\begin{array}{l}\text { HITH } \\
\text { STR. }\end{array}$ \\
\hline $\mathrm{VA}-2$ & VAS - 2 & 2 & 1 & 6 & 10 & $18 \cdot 1 / 2$ & $6-1 / 2$ & $19-3 / 8$ & $7-1 / 4$ & 14 & $6-1 / 8$ & $5.15 / 16$ & $1-3 / 4$ & & 22 & 34 \\
\hline $\mathrm{VA}-2 \frac{1}{2}$ & VAS - $2 \frac{1}{2}$ & $21 / 2$ & 1 & 7 & 12 & $18-5 / 8$ & $5.7 / 8$ & $19 \cdot 3 / 8$ & $6 \cdot 3 / 4$ & 15 & $5 \cdot 5 / 8$ & $6-5 / 8$ & $1-7 / 8$ & 90 & 28 & 40 \\
\hline$V A-3$ & VAS- 3 & 3 & 1 & $7-1 / 2$ & 14 & $20-3 / 8$ & $6-5 / 8$ & $21-1 / 2$ & $7-3 / 4$ & 16 & $6-1 / 4$ & $8-5 / 8$ & $2 \cdot 9 / 16$ & 180 & 36 & 48 \\
\hline VA -4 & VAS - 4 & 4 & $1-1 / 2$ & $9.1 / 2$ & 18 & $24-1 / 2$ & $7 \cdot 3 / 4$ & $25-1 / 2$ & $8 \cdot 3 / 4$ & 20 & $7-1 / 4$ & $10-3 / 4$ & $3 \cdot 1 / 8$ & 310 & 92 & 103 \\
\hline VA- 5 & VAS- 5 & 5 & $1 \cdot 1 / 2$ & 11 & 20 & $27-3 / 4$ & $8.5 / 8$ & $28 \cdot 3 / 4$ & $9 \cdot 5 / 8$ & 23 & $8 \cdot 1 / 8$ & $12 \cdot 3 / 4$ & $3.5 / 8$ & 500 & 122 & 133 \\
\hline $1-6$ & VAS- 6 & 6 & $1-1 / 2$ & $12-1 / 2$ & 24 & $31-1 / 4$ & $9.5 / 8$ & $32-3 / 8$ & $10 \cdot 3 / 4$ & 26 & $9.1 / 8$ & 16 & $4-11 / 16$ & 725 & 156 & 208 \\
\hline VA- 8 & VAS-B & 8 & 2 & 16 & 28 & $39.3 / 8$ & 12 & $40-3 / 8$ & 13 & 33 & $11-3 / 8$ & 20 & $5.11 / 16$ & 1250 & 372 & 384 \\
\hline VA- 10 & VAS- 10 & 10 & 2 & 20 & 32 & $46-7 / 8$ & $13-3 / 4$ & $4 i-7 / 8$ & $14-3 / 4$ & 40 & $13-1 / 8$ & 24 & $6 \cdot 5 / 8$ & 2000 & 526 & 540 \\
\hline $\mathrm{VA}-12$ & VAS -12 & 12 & 2 & 26 & 38 & $57 \cdot 3 / 8$ & 16 & $58-3 / 8$ & 17 & 49 & $15-3 / 8$ & 30 & $8 \cdot 5 / 8$ & 2800 & 805 & 820 \\
\hline
\end{tabular}




\section{ARMSTRONG}

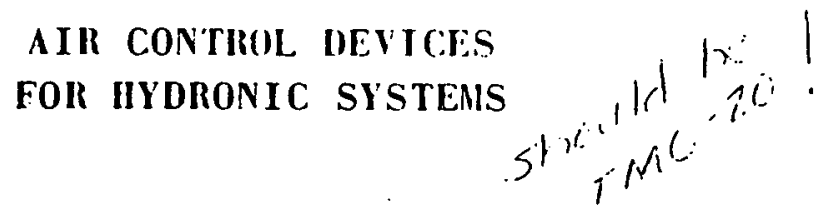

\begin{tabular}{lrr|}
\hline FILE NO.: & 5011.90 \\
DATE: & FCh. $28 / 69$ \\
SUPERSEDES: & 5011.90 \\
DATE: & OCL. & $24 / 60$ \\
\hline$A C$
\end{tabular}

\section{ARMSTRONG BMA BOILER FITTING}

Armstrong BNA Boiler Fittings are made in sizes 1" through 4". The size selected should be the same
as the supply main at the boiler. The Armstrong Boiler Fitting is designed to remove the air at the point of release - the boiler.

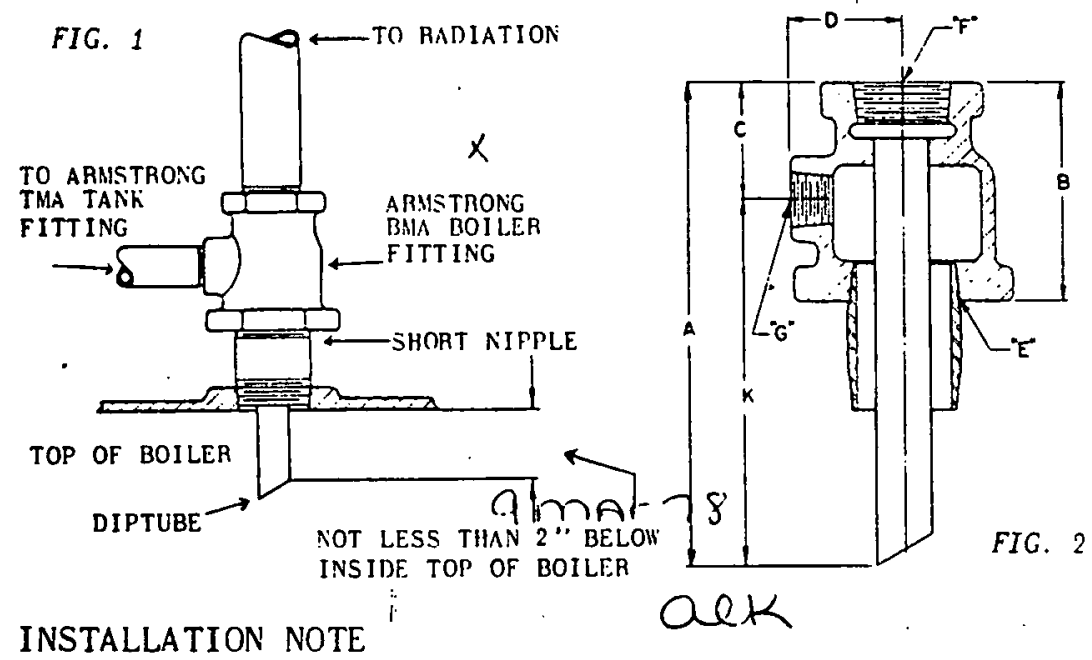

\begin{tabular}{|c|c|c|c|c|c|c|c|c|}
\hline \multirow{2}{*}{$\begin{array}{l}\text { UNIT } \\
\text { NO. }\end{array}$} & \multirow{2}{*}{$A$} & \multirow{2}{*}{ B } & \multirow{2}{*}{$C$} & \multirow{2}{*}{ D } & \multicolumn{3}{|c|}{$\begin{array}{c}\text { PIPE } \\
\text { THREAD }\end{array}$} & \multirow{2}{*}{$k$} \\
\hline & & & & & $E$ & $\mathrm{~F}$ & $G$ & \\
\hline $3 k^{k} 1$ & $10 !$ & 4 & $2 \frac{1}{8}$ & $1 ! 5$ & 2 & I & $\frac{3}{4}$ & 7 \\
\hline$B i k=i$ & $10 \frac{1}{2}$ & 4 & $2 \frac{1}{2}$ & 110 & 2 & $7 \frac{1}{4}$ & $\frac{3}{4}$ & 7 \\
\hline Bivi $1 !$ & $10 !$ & $\therefore$ & $2 \frac{1}{\varepsilon}$ & $1 \frac{1}{1 \vec{k}}$ & 2 & $1 \frac{1}{3}$ & $\frac{3}{4}$ & 7 \\
\hline $8 \div 2$ & $12 \div$ & $4 \frac{1}{2}$ & $2 \frac{1}{c}$ & $2 \frac{1}{2}$ & 3 & 2 & $\frac{3}{4}$ & $8 \frac{1}{3}$ \\
\hline 30142 & $12:$ & $4 \frac{1}{3}$ & $2 \frac{1}{3}$ & $2 \frac{1}{2}$ & 3 & $2 \frac{1}{2}$ & $\frac{3}{4}$ & $8 \frac{3}{3}$ \\
\hline 8. 3 & 143 & $4 \stackrel{5}{4}$ & $2 \frac{3}{2}$ & 3 & 4 & 3 & $\frac{3}{4}$ & $10 \frac{3}{4}$ \\
\hline Buis is & $15 \%$ & $4 \frac{3}{4}$ & $2 \vdots$ & 4 & 6 & 4 & $1 \frac{1}{4}$ & 11. $\frac{3}{4}$ \\
\hline
\end{tabular}

When installing Armstrong BMA Boiler Fittings, care should be taken to cnsure that diptube does not strike any obstruction in boiler. If standard diptube is too long for insertion in boiler, diptube may be cut lo sujt. Dipeube must always be installed below inside top of boiler. See fig. 1.

\section{ARMSTRONG TMA TANK FITTING}

The Armstrong Tank Fitting is designed to maintain the correct amount of air in the expansion tank. When selecting the Armstrong Tank Fitting it is important that the diameter of the.expansion-tank-be... known and a Tank Fitting be selected to suit as follows: -
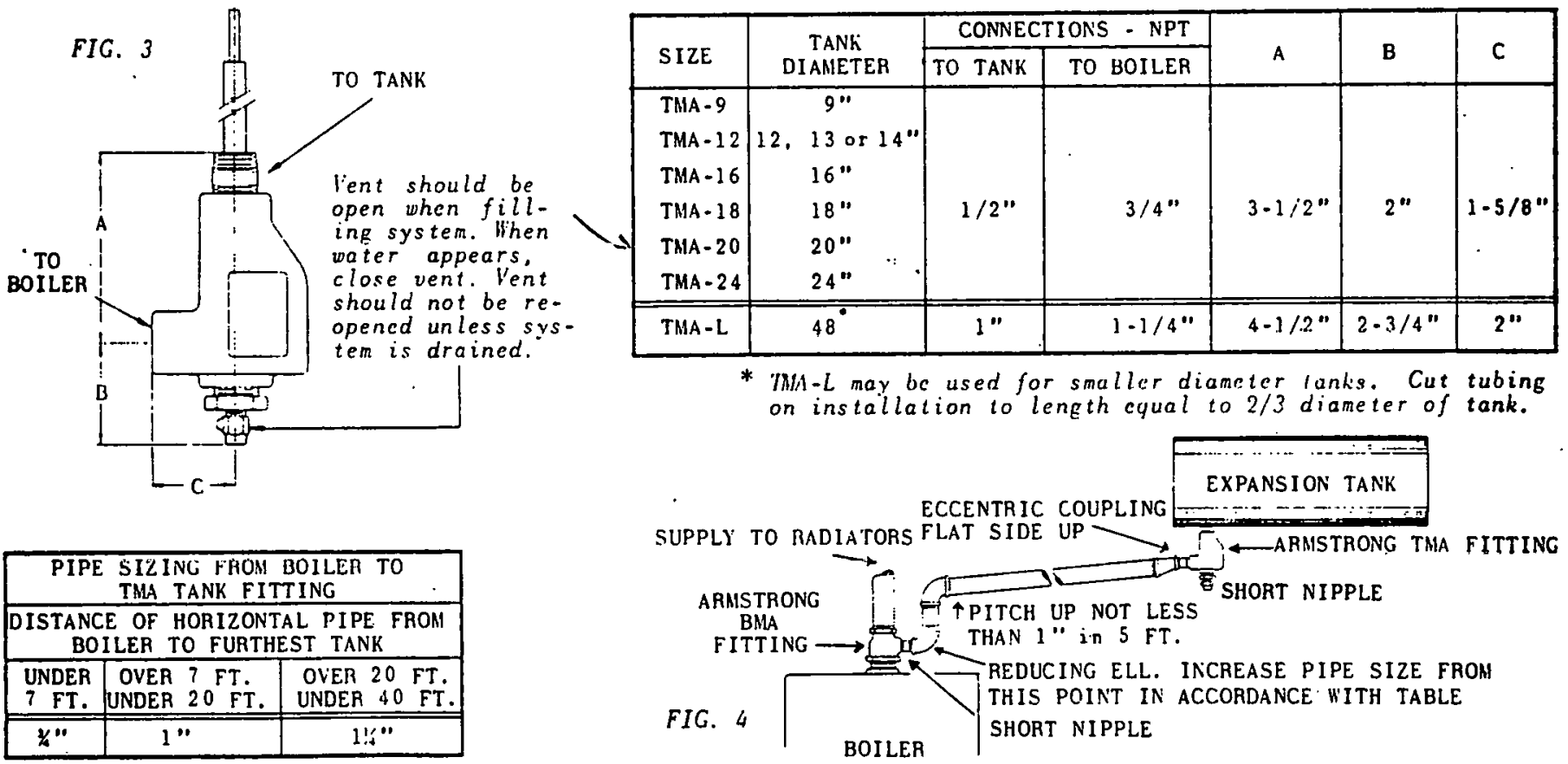

CAUTION:- then using several Armstrong Boiler Fittings on one boiler, only one Boiler Fitting should be connected to a Tank Fitting. Expansion tank connections on remaining Boiler Fittings must be plugged. 


\title{
ARMSTRONG
}

\author{
SUBMITTAL DATA
}

\section{AIH CONTROL DEVICES FOR IYYDIRONIC SYSTEMS}

125 psig WORKING PRESSURE - $250^{\circ} \mathrm{F}$ MAXIMUM OPEIRATING TEMPEMTIURE
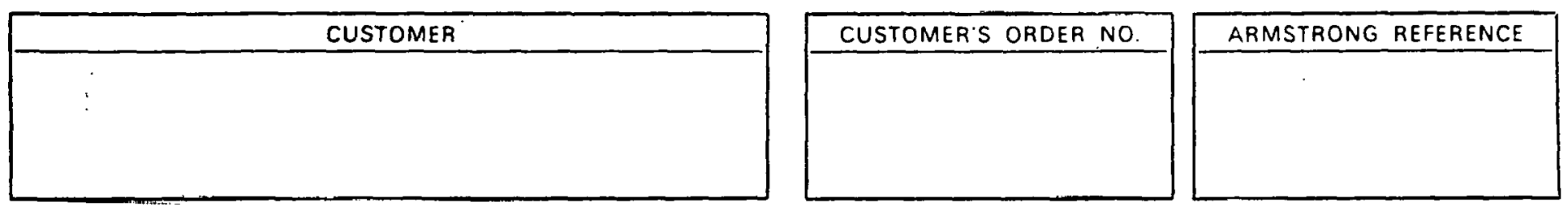

\section{ENGINEER}

JOB REFERENCE

BMA BOILER AIR FITTING

\begin{tabular}{|c|c|c|c|c|c|c|c|}
\hline SIZE & 1 & $1 \frac{1}{4}$ & $1 \frac{1}{2}$ & 2 & $2 \frac{1}{2}$ & 3 & 4 \\
\hline No. Req. & & & & & & & \\
\hline
\end{tabular}

TMA TANK AIR FITTING

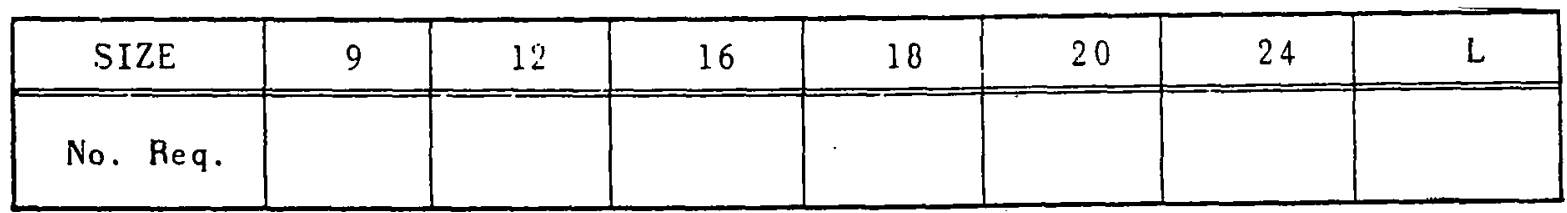

PREPARED BY

DATE : 
WOOD INDUSTRIAL PAODUCTS COMPANY AN ALCO STANDARO COMPANY

Conshohocken, Pa. 19428

TELEPHONE NUMBER
215-825.9110

$215-828.0800$

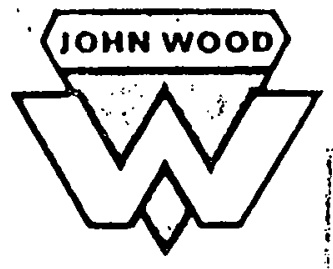

Elleclive duly 1,1976

Form \# 713

SPECIFICATIONS FOR

ASME EXPANSION-COMPRESSION TANKS

125 Lbs. Working Pressure - 188 Lbs. Test Pressure

Painted Red Oxide or Galvanized

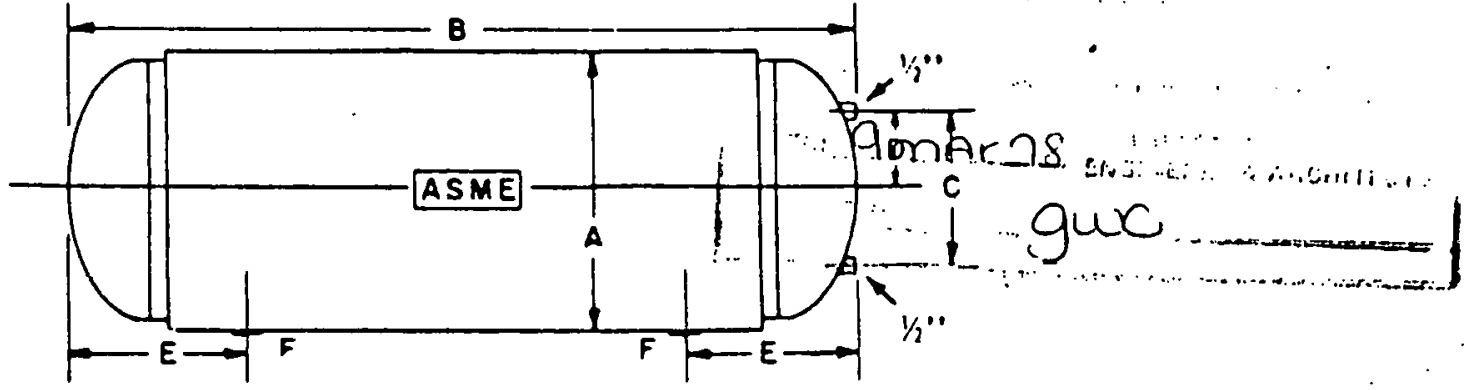

\begin{tabular}{|c|c|c|c|c|c|c|c|c|}
\hline \multirow{2}{*}{ GAL. } & \multirow{2}{*}{ A } & \multirow{2}{*}{ B } & \multirow{2}{*}{ C } & \multirow{2}{*}{$D$} & \multirow{2}{*}{ E } & \multirow{2}{*}{$F$} & \multicolumn{2}{|c|}{ WEICHTS } \\
\hline & & & & & & & PAINTED & GALV. \\
\hline 15 & $12^{\prime \prime}$ & $33^{\prime \prime}$ & $8^{\prime \prime}$ & $4^{\prime \prime}$ & $71 / 2$ & l". & $45 \mathrm{H}$ & $50 \mathrm{H}$ \\
\hline 18 & 12 & 39 & 8 & 4 & $71 / 2$ & 1 & 50 & 60 \\
\hline 24 & 12 & 52 & 8 & 4 & $71 / 2$ & $T$ & 63 & 70 \\
\hline 30 & 14 & 48 & 10 & 5 & $83 / 8$ & $T$ & 65 & 73 \\
\hline 40 & 14 & 63 & 10 & 5 & $8^{3 / 8}$ & 1 & 83 & 93 \\
\hline 60 & 16 & 72 & 12 & 6 & 914 & 1 & 113 & $\begin{array}{l}* 127 \\
\end{array}$ \\
\hline 80 & 20 & $621 / 2$ & 16 & $\overline{8}$ & 10 & $T$ & 130 & .145 \\
\hline 100 & 20 & 78 & 16 & 8 & 10 & 1 & 162 & .181 \\
\hline 120 & 24 & 65 & 20 & 10 & $11:$ & 1 & 195 & 215 \\
\hline 135 & 24 & 72 & 20 & 10 & 111. & 1 & 215 & 235 \\
\hline 180 & 30 & $62 \%$ & 22 & 11 & $13 i_{2}$ & $T 1 \%$ & 295 & $\times 318$ \\
\hline 220 & 30 & 77 & 22 & 11 & $13 !$ & $1 ! 2$ & 355 & 383 \\
\hline 235 & 30 & $81^{1 / 2}$ & 22 & 11 & $1.3 \frac{1}{2}$ & $\frac{1}{12}$ & 385 & पा5 \\
\hline 310 & 30 & 105 & 22 & 11 & $13 \%$ & $1 ! 2$ & 480 & 517 \\
\hline 300 & 36 & $71: 4$ & 28 & 14 & $14^{3 / 4}$ & $11 / 2$ & 485 & 515 \\
\hline 400 & 36 & $93 \frac{1}{2}$ & 28 & 14 & $14^{3 / 4}$ & $11 \%$ & 634 & 675 \\
\hline 515 & 36 & $120^{3}$ & 28 & 14 & $14^{3 / 4}$ & $\frac{1}{11 / 2}$ & 789 & 840 \\
\hline 525 & 42 & 97 & 2 Sets & $15^{\prime \prime C} \mathrm{Ctr}$. & $14^{7 / 16}$ & 2 & 834 & 882 \\
\hline 750 & 42 & 136 & 2 Sets & $15^{\prime \prime} \mathrm{Ctr}$. & $14^{7 / 16}$ & 2 & $\pi 33$ & 1200 \\
\hline 1000 & 48 & 129 & 2 Sets & $18^{\prime \prime} \mathrm{Ctr}$ & $15^{3 / 4}$ & 2 & 1729 & 1814 \\
\hline 1500 & 48 & 198 & 2 Sets & $18^{\prime \prime} \mathrm{Ctr}$. & $15^{3 / 4}$ & 2 & 2319 & 2421 \\
\hline 2000 & 48 & 267 & 2 Sets & $18^{\prime \prime} \mathrm{Ctr}$ & $15^{3 / 4}$ & 2 & 2914 & 3025 \\
\hline
\end{tabular}

- Sizes mormally in stock for prompt shipment.

Tonks ore fabricated to ASME Code, subject to approval of o resident, licensed inspector.

Telltale holes are furnished in lieu of inspection opening on oll sizes thru 15 to 144 gallon. Two 2" plugged in. spection openings ore provided on sizes 180 thru 515 gollon. 525 gollon and larger are equipped with an $11 \times 15^{\prime \prime}$ monhole.

Taps for 2 overlapping gauge glass ossemblies ore included on 525 gallon size \& larger.

Guarantees are agoinst defects in moterial or workmanship, and are good up to the time of installation and test only. The guarantee is to the extent of the tonk only, and does not include ony allowarice for replacement or consequentiol damage. Material must not be returned without first receiving our permission.

B -7 


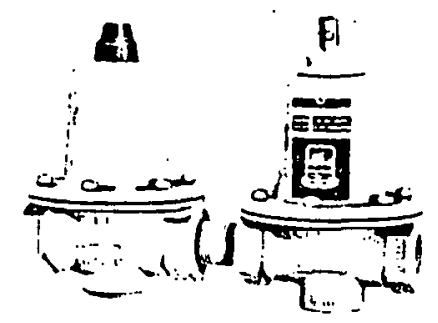

\section{COMBINATION UNITS}

Note: Reducing Valve factory set at 12 psi, adjustable 7 to 25 psi. Reducing Valve equipped with strainer, anti-syphon check, composition disc and brass valve scat.

\begin{tabular}{|c|c|c|c|c|}
\hline $\begin{array}{c}\text { NODEL } \\
\text { NO. }\end{array}$ & SIZE & $\begin{array}{c}\text { RELIEF } \\
\text { SETTING }\end{array}$ & BODY & $\begin{array}{c}\text { APPROX. } \\
\text { SIIIPPING WT. }\end{array}$ \\
\hline \hline C- 11 & $\% "$ & 30 PSI & BRASS & $2 \%$ LBS. \\
\hline C-20 & $1 \% "$ & 30 PSI & IRON & 6 LBS. \\
\hline
\end{tabular}

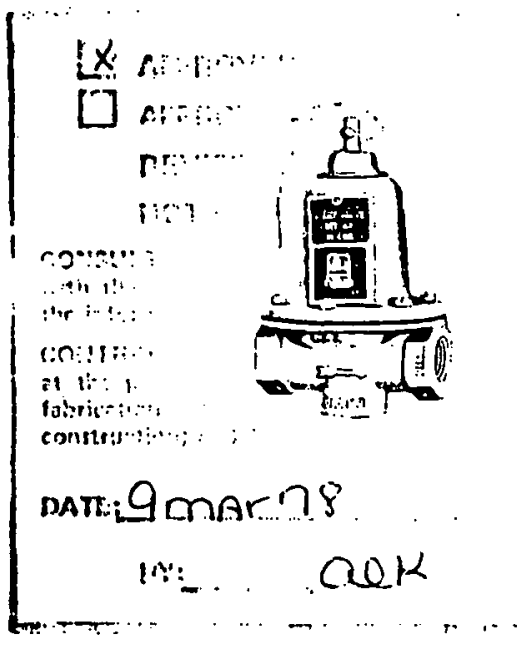

RELIEF VALVES

\begin{tabular}{|c|c|c|c|c|}
\hline $\begin{array}{c}\text { MODEL } \\
\text { NO. }\end{array}$ & SIZE & $\begin{array}{c}\text { RELIEF } \\
\text { SETTING }\end{array}$ & BODI & $\begin{array}{c}\text { APPROX. } \\
\text { SHIPPING WT. }\end{array}$ \\
\hline \hline RL-1] & $1 / 2 "$ & 30 PSI & BRASS & $11 / 4$ LBS. \\
\hline RL-20 & $1 / 2$ & 30 PSI & IRON & 3 LBS. \\
\hline
\end{tabular}

\section{REDUCING VALVES}

Note: RD-11 and RD-20 Reducing Valve same as used in C-11 and C-20 Combination Units. All Units complete with strainer and anti-syphon check.

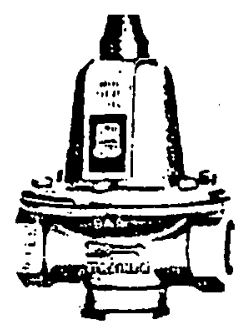

\begin{tabular}{|c|c|c|c|c|}
\hline $\begin{array}{l}\text { MODEL } \\
\text { NO. } \\
\end{array}$ & SIZE & $\begin{array}{l}\text { STD. } \\
\text { DEL. SETTING }\end{array}$ & BODY & $\begin{array}{c}\text { APPROX. } \\
\text { SHIPPING } W T \text {. }\end{array}$ \\
\hline $\mathrm{RD}-11$ & $1 / 2 "$ & $12 \mathrm{PSI}^{*}$ & BRASS & $11 / 4$ LBS. \\
\hline $\mathrm{RD}-20$ & $1 / 2 "$ & $12 \mathrm{PSI} *$ & IRON & $31 / 4$ LBS. \\
\hline RD- 50 & $3 / 4$ & 12 PSI & IRON & $31 / 4$ LBS. \\
\hline HRD -60 & $\frac{1}{2}=$ & 45 PSI + & BRASS & $31 / 4$ LBS. \\
\hline HRD - 70 & $3 / 4 "$ & 45 PSI + & BRASS & $3 \frac{1}{4}$ LBS. \\
\hline
\end{tabular}

* Adjustable 7 PSI to 25 PSI.

+ Adjustable 25 PSI to 125 PSI. Maximum Initial Pressure 150 PSI. 
JOIB : -

ENG I NEER : -

CONTRACTOR : -

onter No. IJA TE

QUOTATION REFFRENCE: -

REPRESENTATIVE: -

COMBINATION UNITS

\begin{tabular}{|c|c|c|c|}
\hline \multirow{2}{*}{$\begin{array}{c}\text { NOIDEI. } \\
\text { NO. }\end{array}$} & \multicolumn{2}{|c|}{ STD. SETTTNGS } & NO. \\
\cline { 2 - 4 } RED. * & REI,IEF & REQ \\
\hline C- 11 & $12 \mathrm{PSI}$ & $30 \mathrm{PSI}$ & \\
\hline C- 20 & $12 \mathrm{PSI}$ & $30 \mathrm{PSI}$ & \\
\hline
\end{tabular}

* Adjustable 7 PSI to 25 PSSI.

\section{RELIEF VALVES}

\begin{tabular}{|c|c|c|}
\hline $\begin{array}{c}\text { MODEL } \\
\text { NO. }\end{array}$ & $\begin{array}{c}\text { RELIEF } \\
\text { SETTING }\end{array}$ & $\begin{array}{c}\text { NO. } \\
\text { REQ }\end{array}$ \\
\hline \hline RL-11 & 30 PSI &. \\
\hline RL-20 & 30 PSI & \\
\hline
\end{tabular}

REDUCING I'ALIES

\begin{tabular}{|c|c|c|}
\hline $\begin{array}{c}\text { NODEL } \\
\text { NO. }\end{array}$ & $\begin{array}{c}\text { STI). DEL. } \\
\text { SETTING }\end{array}$ & $\begin{array}{c}\text { NO. } \\
\text { REQ' D. }\end{array}$ \\
\hline \hline RD-11 & 12 PSI & \\
\hline RD-20 & 12 PSI & \\
\hline RD-50 & 12 PSI & \\
\hline \hline HRD-60 & 45 PSI & \\
\hline HRD- 70 & 45 PSI & \\
\hline
\end{tabular}

+ RD-11, RD-20 and RD-50 Adjustable 7 PSI to 25 PSI.

HRD-60 and HRD-70 Adjustable 25 PSI to 125 PSI. Maximum Initial Pressure 150 PSI. 
WATTS

\section{A-740 SERIES ASME WATER PRESSURE RELIEF VALVES}

for Pressure Protection of Hot Water Heating Boilers

\section{Sizes: $3 / 4^{\prime \prime}$ thru $2^{\prime \prime}$}

The 174A-740 Series was developed to offer a complete line of boiler safety relief valve sizes from $3 / 4 "$ through 2" inclusive and with corresponding high BTU discharge capacity ratings. Watts was the first to offer this full selection of sizes, which afford complete pressure protection for the great majority of all hot water heating and supply boilers with a single valve.

Sizes 1" to 2" inclusive are proportionately larger valves to the $3 / 4 "$ size. They are designed for larger institutional and industrial installations to protect high BTU rated boilers that need greater relief capacities.

Whenever plans call for the latest and finest in A.S.M.E. relief valves, you'll find them in the Watts line.

\section{MFEATURES : : T}

- Seat located above drain: water can't be trapped and sediment can't foul seat

- Non-mechanical seat-to-disc alignment will not stick or freeze

- Water seal of high temperature resisting material isolates spring working parts from water during relief

- No. 740 has the same design features as No. 174A except for difference in body construction and material

\section{SPECIFICATIONS}

\section{BOILER RELIEF VALVES}

Each hot water space heating boiler shall be equipped with a pressure relief valve set to relieve below the maximum boiler working pressure. The device shall be certified in accordance with the A.S.M.E. low pressure heating boiler code Section 4. The BTU rating of the valve must be in excess of the BTU output heating of the boiler. Watts Regulator Company Series $174 \mathrm{~A}, 740$ or equal.

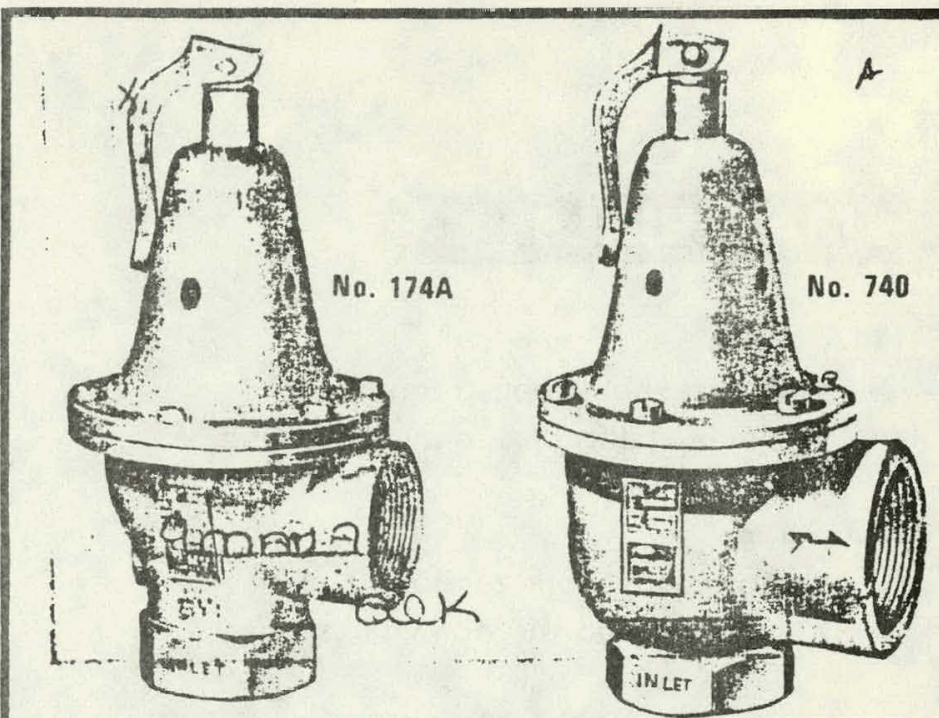

OPERATION: A hot water heating boiler operates normally full of water and steams only when there is trouble with the firing controls. When this occurs, it is good "safety" procedure to reduce the energy stored in the boiler by lowering the heat content of the boiler as rapidly as practicable.

WATER DISCHARGE

As thermal expansion conditions develop, pressures may be built up to the setting of the relief valve. In this phase of operation, it acts as a water relief valve, discharging the small quantity of water which is expanded in the system.

STEAM DISCHARGE

Should operating controls fail, permitting run-away firing, the boiler water may reach steamforming temperatures, creating a steam pressure condition. Acting as a steam safety valve, it dis. charges steam at the rate or faster than the boiler can generate it, thus restoring system pressure to a safer level.
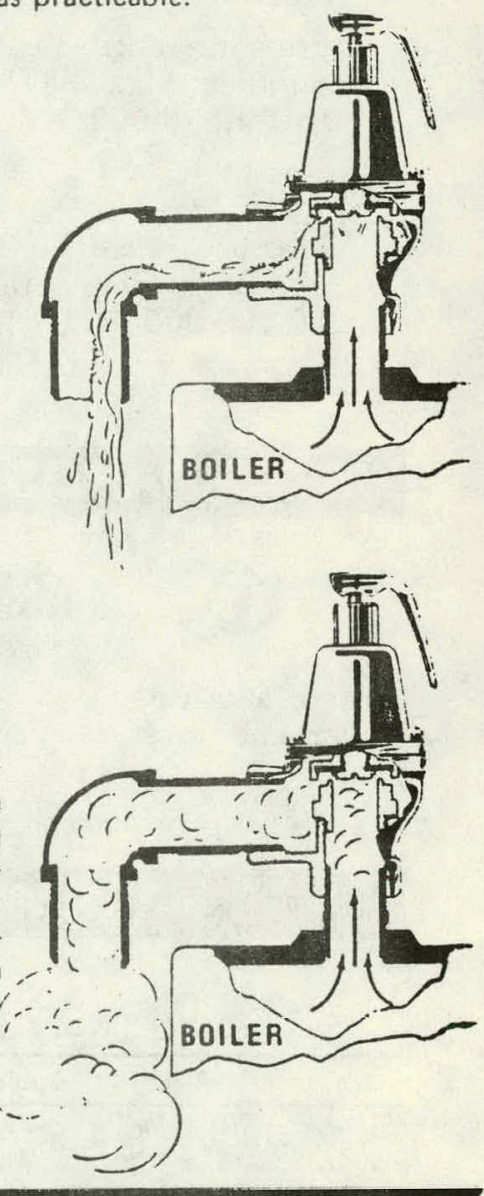

DESIGNED AND MANUFACTURED BY:

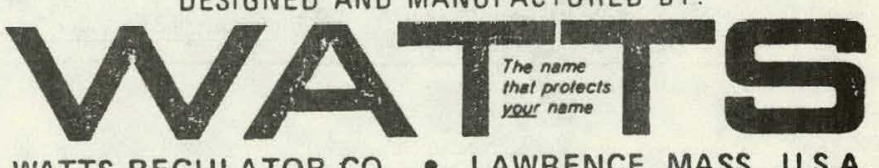

WATTS REGULATOR CO. - LAWRENCE, MASS., U.S.A.

With Representatives in Over 40 Cities from Coast to Coast

$$
\text { IN CANADA }
$$

WATTS REGULATOR OF CANADA, LTD. WESTON, ONTARIO 


\section{WATERIALS:}

174A Series

- Bronze body construction

- Non-metallic disc-to-metal seating

740 Series

- Iron body construction

- Non-metallic disc-to-metal seating

\section{MPRESSUREX}

\section{A Series}

Pressure range $30 \mathrm{lbs}$. to $160 \mathrm{lbs}$. with corresponding high BTU/HR ratings from 650,000 to $14,370,000 \mathrm{BTU} / \mathrm{HR}$.

\section{Series}

Pressure range $30 \mathrm{lbs}$. to $75 \mathrm{lbs}$. with corresponding high ratings from 925,000 to $10,700,000 \mathrm{BTU} / \mathrm{HR}$.

\section{STANDARDS}
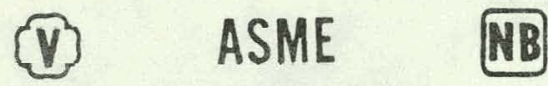

Tested and rated by A.S.M.E. National Board of Boiler and Pressure Vessel Inspecturs.

\section{CAPACITY}

\section{No. 174A Series}

SETTINGS and RELIEVING CAPACITIES

(National Board Certified Ratings)

BTU Steam Discharge Capacities

\begin{tabular}{|c|c|c|c|c|}
\hline Size & 30 lbs. & $100 \mathrm{lbs}$. & 125 lbs. & 150 lbs. \\
\hline $3 / 4$ & 650,000 & $1,695,000$ & $2,070,000$ & $2,445,000$ \\
\hline $1^{\prime \prime}$ & $1,005,000$ & $2,635,000$ & $3,215,000$ & $3,795,000$ \\
\hline $1 y^{\prime \prime}$ & $1,865,000$ & $4,870,000$ & $5,945,000$ & $7,020,000$ \\
\hline $1 y_{2}^{\prime \prime}$ & $2,020,000$ & $5,290,0 \cup 0$ & $b, 4 \mathrm{bU}, \mathrm{UUU}$ & $7,630,000$ \\
\hline $2^{\prime \prime}$ & $3,815,000$ & $9,970,000$ & $12,170,000$ & $14,370,000$ \\
\hline
\end{tabular}

NOTE: We recommend No. 740 Series as best buy for hot water space heating boiler requirements between 30 through $75 \mathrm{lbs}$.

\section{No. 740 Series}

- SETTINGS and RELIEVING CAPACITIES

(National Board Certified Ratings) BTU Steam Discharge Capacities

\begin{tabular}{|c|c|c|c|c|}
\hline Size & 0 lbs. & 45 lbs. & 50 lbs. & 75 lbs. \\
\hline $9 / 4=1 "$ & 925,000 & $1,245,000$ & $1,352,000$ & $1,886,000$ \\
\hline $1^{\prime \prime} \times 11 / 4$ & 0 & 0 & 00 & \\
\hline $11 / 4 " \times 1 \frac{1}{2 \prime}$ & & & $3,075,000$ & $4,285,000$ \\
\hline $11^{\prime \prime} \times 2^{\prime \prime}$ & $2,900,000$ & $3,903,000$ & 4,238 & $5,910,000$ \\
\hline $2^{\prime \prime} \times 21_{2}^{\prime \prime}$ & $5,250,000$ & $7,050,000$ & $7,650,000$ & $10,700,000$ \\
\hline
\end{tabular}

NOTE: Valve settings, other than shown above, are available in $5 \mathrm{lb}$ increments between the pressure range of 30 through $75 \mathrm{lbs}$.

\section{WDIMENSIONS :-WEIGHT}

No. 174A Series

\begin{tabular}{|c|c|c|c|c|c|}
\hline No. & Size & Model & Height & Length & Weight \\
\hline $174 A$ & $3 / 4 " \times 3 / 4 "$ & M3 & $5^{1 / 81}$ & $21 / 2^{\prime \prime}$ & $11 / 2 \mathrm{lbs}$. \\
\hline $174 \mathrm{~A}$ & $1^{\prime \prime} \times 1^{\prime \prime}$ & M1 & $53 / 4 "$ & $3^{\prime \prime}$ & $3^{1 / 8} \mathrm{lbs}$. \\
\hline $174 \mathrm{~A}$ & $11 / 4 " \times 11 / 40$ & M & $83 / 8^{\prime \prime}$ & $43 / 4^{\prime \prime}$ & $61 / 4 \mathrm{lbs}$. \\
\hline $174 \mathrm{~A}$ & $11 / 2 " \times 11 / 2 "$ & M & $9^{\prime \prime}$ & $47 / 8=$ & $71 / 4$ lbs. \\
\hline $174 \mathrm{~A}$ & $2^{\prime \prime} \times 2^{\prime \prime}$ & M & $115 / 8 "$ & $61 / 4^{\prime \prime}$ & $13^{3 / 4} \mathrm{lbs}$. \\
\hline
\end{tabular}

No. 740 Series

\begin{tabular}{cccccc}
\hline No. & Size & Model & Height & Length & Weight \\
\hline 740 & $3 / 4^{\prime \prime} \times 1^{\prime \prime}$ & MI & $55 / 8^{\prime \prime}$ & $3^{\prime \prime}$ & $17 / 8$ lbs. \\
740 & $1^{\prime \prime} \times 11 / 4^{\prime \prime}$ & M & $71 / 4^{\prime \prime}$ & $31 / 2^{\prime \prime}$ & $31 / 8$ lbs. \\
740 & $11 / 4^{\prime \prime} \times 11 / 2^{\prime \prime}$ & M & $83 / 4^{\prime \prime}$ & $45 / 8 "$ & $61 / 8$ lbs. \\
740 & $11 / 2^{\prime \prime} \times 2^{\prime \prime}$ & M & $91 / 4^{\prime \prime}$ & $51 / 4^{\prime \prime}$ & $71 / 2 \mathrm{lbs}$. \\
740 & $2^{\prime \prime} \times 21 / 2^{\prime \prime}$ & M & $115 / 8^{\prime \prime}$ & $63 / 4^{\prime \prime}$ & $161 / 2 \mathrm{lbs}$. \\
\hline
\end{tabular}
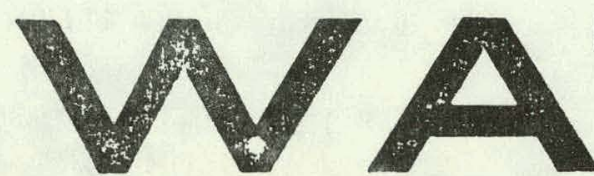

ES.174A.740 761
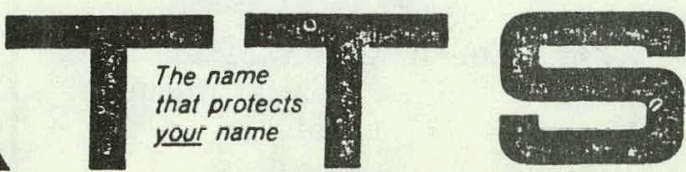

LAWRENCE, MASS., U.S.A. 


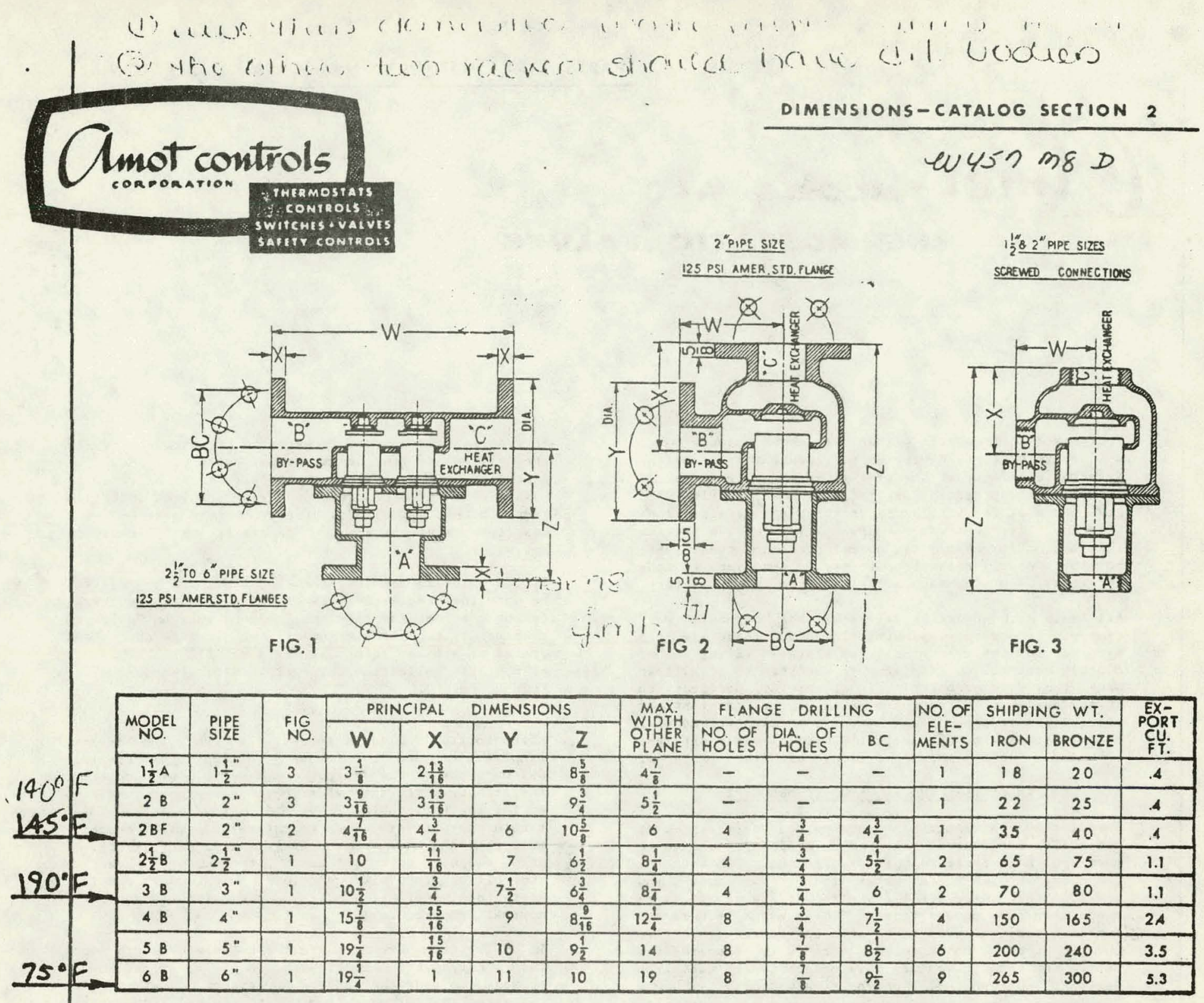

BASIC MODELS 1-1/2A, 2B AND 2BF ARE AVATLABLE IN CAST IRON OR ALL BRONZE. BASIC MODELS 2-1/2B THROUGH 6B ARE AVAILABLE IN CAST IRON, DUCTILE IRON OR ALL BRONZE. CAST IRON AND DUCTILE IRON VALVES ARE BRONZE FITTED.

VALVES MAY BE ORDERED BY SPECIFYING THE BASIC MODEL NO., THE HOUSING MATERIAL DESIRED, THE NOMINAL TEMPERATURE DESIRED, AND ANY SPECIAL CONDITIONS THE VALVE MUST WITHSTAND. AMOT IS ADOPTING THE POLICY OF ADDING TO THE COMPLETE MODEL NUMBER, A LETTER DESIGNATING ANY SPECLAL HOUSING FEATURES, ANOTHER LETTER TO DESIGNATE HOUSING MATERIAL (C, B, D OR A, ETC.) AND A CODE NUMBER AFTER THE NOMINAL TEMPERATURE TO INDICATE TYPE OP ELEMENT. THUS A VALVE ORDERED AS A 2-1/2B-160 CAST IRON BRONZE FITTED, WILI BE STAMPED ON THE NAMEPLATE AS A 2-1/2BOC160-01. THE O MEANS SIANDARD CONSTRUCTION, C MEANS CAST IRON, THE 01 MEANS STANDARD 1096X- TYPE ELEMITNT SET TO MAINTAIN A NOMINAL $160^{\circ} \mathrm{F}$. FLUID TEMPERATURE.

AMOT THERMOSTATIC VALVES ARE TEMPERATURE RATED FOR THE EXPECTED NOMTNAL OPERATING TEMPERATURE IN JAGKET WATER SERVICE. ON LUBRICATING OIL APPLICATIONS, THE OPERATING TEMPERATURE MAY BE SLIGHTLY ABOVE THE NOMINAL RATING DEPENDING ON THE TYPE OF OIL, FLOW RATE, AND OTHER CONDITIONS OF THE SYSTEM.

STANDARD STOCK TEMPERATURE SETTINGS AVAILABLE FOR AGB SERIES VALVES ARE $75,90,105,120,130,140,150,155,160$, $165,170,180$ AND $195^{\circ} \mathrm{F}$. SOME OTHER TEMPERATURE SEITINGS AVAILABLE ON SPECIAL ORDER ON LIMITED STOCK BASIS. BASIC MODEL 1-1/2A REQUIRES ONE 1126X-(TEMP.) TYPE THERMOSTATIC ELEMENT. BASIC MODEL B REQUIRES THE 1096X(TEMP.) TYPE ELEMENTS WHICH ARE INTERCHANGEABLE ON 2" THROUGH 6" SIZE.

2" TO 6" TYPE B VALVES ARE AVAILABLE WITH NAVY FLANGE DRILIING.

CERTIFIED PRINT

CUSTOMER

BY

PURCHASE ORDER NO.

DATE

\section{AMOT CONTROLS CORPORATION}

OUTLINE DIMENSIONS MODELS A \& B THERMOSTATIC VALVES
FORM 121

REV. 8

DATE $\quad 7.70$ PAINTED IN U. 3. A 


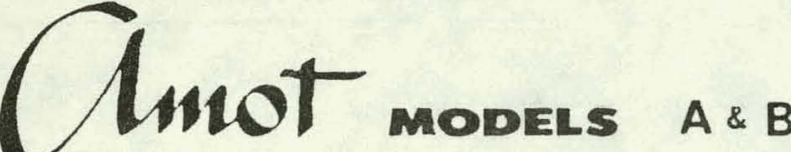

\section{THERMOSTATIC VALVES}

Your Amot Thermostatic Valve has been manufactured with extreme care and tested to insure that it had no detectable defects at the time it left the factory. If the value is correctly applied and installed it wlll give years of service under reasonable operating conditions. This instruction manual will give you service information for nearly all normal operating conditions, but for unusual situations it may be necessary to contact your Amot representative or the Amot factory.

A11 Amot A \& B series values use the "expanding wax" type of temperature sensing element. These elements are set to their nominal temperature rating under closely controlled conditions, and cannot be altered once they have been set. If it is ever necessary to change the nominal rating of a valve, different set of elements must be used. Amot has an element exchange program for new or only slightly used elements-see Form 138 for terms and conditions of the exchange.

\section{INSPECTING THE VALVE ON ITS RECEIPT}

Immediately on receiving your valve, check it over carefully for damage received in shipping, and to be sure you have recelved the proper unit. In checking the model number of the valve against your order, you may find that there are a few more digits on the nameplate than show on the order. These extra letters and numbers merely helf us to be able to identify the valve and its type of construction more fully than in the past. For example you may have ordered a $2 B F$ Cact Iron Valve at $160^{\circ} \mathrm{F}$ nominal, but you received a 2BFC-160-01. The larger number means the valve is a $2 \mathrm{BF}$ type, with cast iron housing, $160^{\circ} \mathrm{F}$ nominal rating; and the -01 indicates standard $1096 \mathrm{X}$ type elements were used. Similarly, a $1 \frac{2}{2} A-170$ (available in bronze only) becomes $1 \frac{1}{2} A O B-170-01$. The first 0 means standard $1 \frac{1}{2}$ "NPT connection threads, $B$ means bronze and the 01. Indicates standard $1126 \mathrm{X}$ elements.

\section{INSTALLATION}

$A \& B$ Valve dimensions are given on Form 121 , a certified copy of which is supplied with each valve order, and which should be checked against the actual valve on receipt of the order. If special engineered drawings have been prepared, these drawings should be followed. Any conflict arising between such drawings and Amot standard instructions should be resolved before the valve is put into service.

On Page 3 of this bulletin are several recommended methods of applying Amot valves. In general, "diverting" system will provide slightly more even temperature contral than a "mixing" arrangement. This is because the former introduces a homogenous fluid to the sensing elements whereas a mixing system requires the fluid at two different temperatures to $\mathrm{mix}$ in the small volume of the valve.
If severe electrolysis is expected or encountered in the system, a zinc or magnesium waste plug should be installed in the valve at the $A$ port - or as close as pussiblic to it. For direct oen warer rnnled installations, bronze valves with plated elements must be used. Cast iron housings are not generally satisfactory on sea water.

If the valve is mounted at the high point of sny system, be sure that the system is properly vented to prevent trapping air at the elements. A good rule to follow on systems is to place air vents at all high points and Utalus at all low points. Vente can all be connected to a single collection point if feasible. as shown in Fig. 1, Page 3.

\section{OPERATION}

After initially placing the valve in operation, system temperatures should be closely watched to be sure that all portions of the circuit are performing properly. A system in which the valves have been properly selected for the anticipated flows and heat rejection rates should operate very close to the value nominal temperature rating. Water cooling systems will usually operate at or slightly below the nominal temperature. Lubricating oil or other viscous fluids with lower specific neats than water will indicate at or slightly above the nominal temperature.

In any system where the indicated temperatures are more than $5^{\circ} \mathrm{F}$ frum the nominal valve rating, an effort should be made to find the cause. Any system operatiug at an indicated $10^{\circ} \mathrm{F}$ or mure from the anticipated temperatures could bein trouhle and the cause must be lucated immediately. Standard Amot elements should not be operated continuously at temperatures in excess of $25^{\circ} \mathrm{F}$ above their nominal rating. On most Amot elements, $10^{\circ} \mathrm{F}$ above the nominal is the maximum stroke point of the element sliding valve, and beyond that point the valve is directing all of the flowing fluid to the $C$ Port.

The assembled-unit type of construction of the Amot elements make them easy to check if they are suspected of causing erroneous system operation. The following procedure will give you an indication as to whether the element is close to its proper calibration. (For an accurate test, we suggest the elements be shipped prepaid to Amot for checking in our calibrated test tanks. Notc! Whenever shipping elements, be sure to wrap them individually for protection against nicks and bumps.)

On most elements in the $A \& B$ valve series, the sliding valve starts to lift off the spider seat at $5^{\circ} \mathrm{F}$ below the nominal temperature rating stamped on the side strap. (This is the only number referencing temperature on the element. All others are production control numbers.) At $10^{\circ} \mathrm{F}$ above the nominal, the element is at or near its full stroke. 


\section{TROUBLE-SHOOTING}

Place the element in a bucket of water $10^{\circ} \mathrm{F}$ below the nominal rating and stir the water vigorously with the element for 5 minutes (the sliding valve should not be off its seat). Next, place the element in water at $15^{\circ} \mathrm{F}$ above the nominal rating and stir vigorously for 5 minutes. The element should now be fully stroked. This is determined by immediately placing the element back in the valve housing, and pushing the element spider fully into its counterbore. If the resistance of the sliding value overtravel spring can be felt, the element is fully stroked. Perform the last step quickly before the element has cooled, and DO NOT USE OII AS THE TEST BATH. On very high temperature elements, water and glycol may be used.

\section{MAINTENANCE}

Amot thermostatic valves probably require less maintenance than any other type for similar use. Elements in normal service should be good for 6 to 10 years. Excessive temperatures, chemical, electrolytic or cavitation attack will of course shorten the life of the elements and seats (which are replaceable). Water additives may cause swelling of the 0-ring seal around the sliding valve, to the point where they may affect element action and require replacement. Synthetic base lubricants will definitely attack the 0 -ring seals which may be removed, or replaced by rings of alternate material.

Carbonates, scale and other solids must not be permitted to build up on sliding valve or sensing cup surfaces. The valve and elements may be cleaned with mild acid or Oakite solutions. Hard scale may require wire brush buffing. Unless definite trouble is encountered in operation, a valve need not he inspected morc than eveiy 2 to 3 years. When replacing elements, a light coating of grease on the 0-ring is helpful in re-assembly. Be sure the 0 -ring is centered in the sleeve before replacing element. When ordering replacement parts for Amot valves, always give the item number, name of the part, the complete valve model number and the correct oerial number.

Amot does not recommend that a large stock of spare parts be maintained at the valve installation. Most commonly used elements and seals are immediately available from Amot $B^{\prime}$ stocking area representatives, or from the factory direct. Rubber seals and composition gaskets are rated for a shelf life of one year from date of shipment. If adequately sealed from air, they may be good for longer periods. Shelf life of the Amot elements is from one to two years, depending on storage conditions.

\section{COMYNLY USED REPLACEMENT PARTS}

BASIC

MODBI

NO. OF
ELEMENTS
$\&$ O RING

PER VALVE

1)A (Older)

$1 \frac{1}{2} A$ (Current)

$1 \frac{1}{2} B 2 B 2 B P(01 d e r)$

$1 \frac{1}{2}$ 2B 2BF (Current)

$2+B$

3 B

4 B

5 B

6 B

\begin{tabular}{c} 
BASIC \\
ELEMENT \\
PART \\
NO. \\
\hline
\end{tabular}

$1126 \mathrm{X}$ - (Temp.)

$1126 \mathrm{X}$ - (Temp

1096X-(Temp.)

1096X-(Temp.)

1096X - (Temp.)

$1096 \mathrm{X}$-(Temp

1096X-(Temp.)

1096X-(Temp.)

1096X - (Temp.)
- RING HOUSING

ELEMENT GASKET

SEAL PART

NO. NO.

$1919 \quad 1067$

$1919 \quad 76$

$1183 \quad 1100$

$1183 \quad 761$

$1183 \quad 1094$

$1183 \quad 1094$

$1183 \quad 1123$

$1183 \quad 1117$

$1183-1149$
In the event that your cooling system does not operate close to the desired temperature, the following check list may point to one or more causes for the problem.

1. SYSTEM TEMPERATURE TOO COLD.

A. Insufficient heat rejected to coolant to maintain temperature.

B. Wrong nominal temperature selected.

C. Bi-metallic type thermometers will indicate low when calibrated in oil, then used in water.

D. Thermostatic valve greatly oversized or cooliny capacity of system much greater than required.

E. Thermostatic valve installed backwards, forces water to cooler at low temperatures.

F. Worn 0-ring seal or broken old style lip-type seal (see Form 542).

G. Too great a pressure difference (in excess of 25 psi) berween valve porto.

H. Foreign material stuck between sliding valve and seat.

2. SYSTEM TEMPERATURE TOO HOT.

A. Cooling capacity of system not adequate.

B. Thermostatic value too small for flow rate (also causes high pressure drop and possibly cavitatiou).

C. Valve installed backwards--as temp. increases, $B$ port closes, reducing flow to cooler.

D. Bypass will not close due to worn or pitted seats, sliding valve, 0-ring seal, etc.

E. Element may have been over-temperatured sufficiently to affect calibration or rupture wax seal. Requires complete now element.

F. Solids buildup on element sliding valve prevents proper action of element.

G. Foreign material stuck between sliding valve and seat.

H. Excessive pressure differential between ports (very low pressure through bypass leg-very high pressure in cooler).

\section{ADDITIONAL ITEMS THAT MAY BE CHECKED ARE--}

A. Thermometers--A thermometer that reads the same whether system is cold or hot needs replacing.

B. Location of thermometers--On horizontal pipe runs, these should be in the side of the pipe when posible. (Particularly on oil systems.)

c. They should be as far as possible downstream from the confluence of two streams of different temperature.

D. Look for bypasses or "sneak circuits" which prevent thermostatic valve control of the complete system.

E. Check to see that valve is marked properly.Lookin the $B$ port, one should always see the top of the element sliding valve (see section on Page 4).

F. Cracked or broken valves may be due to trying to make up piping runs that are too short; using small ring-type gaskets on flat face cast iron flanges and pulling bolts too tight; lack of expansion isolation between valve and piping; excessive weight or mis-alignment of piping; allowing untreated water to freeze in the system. 


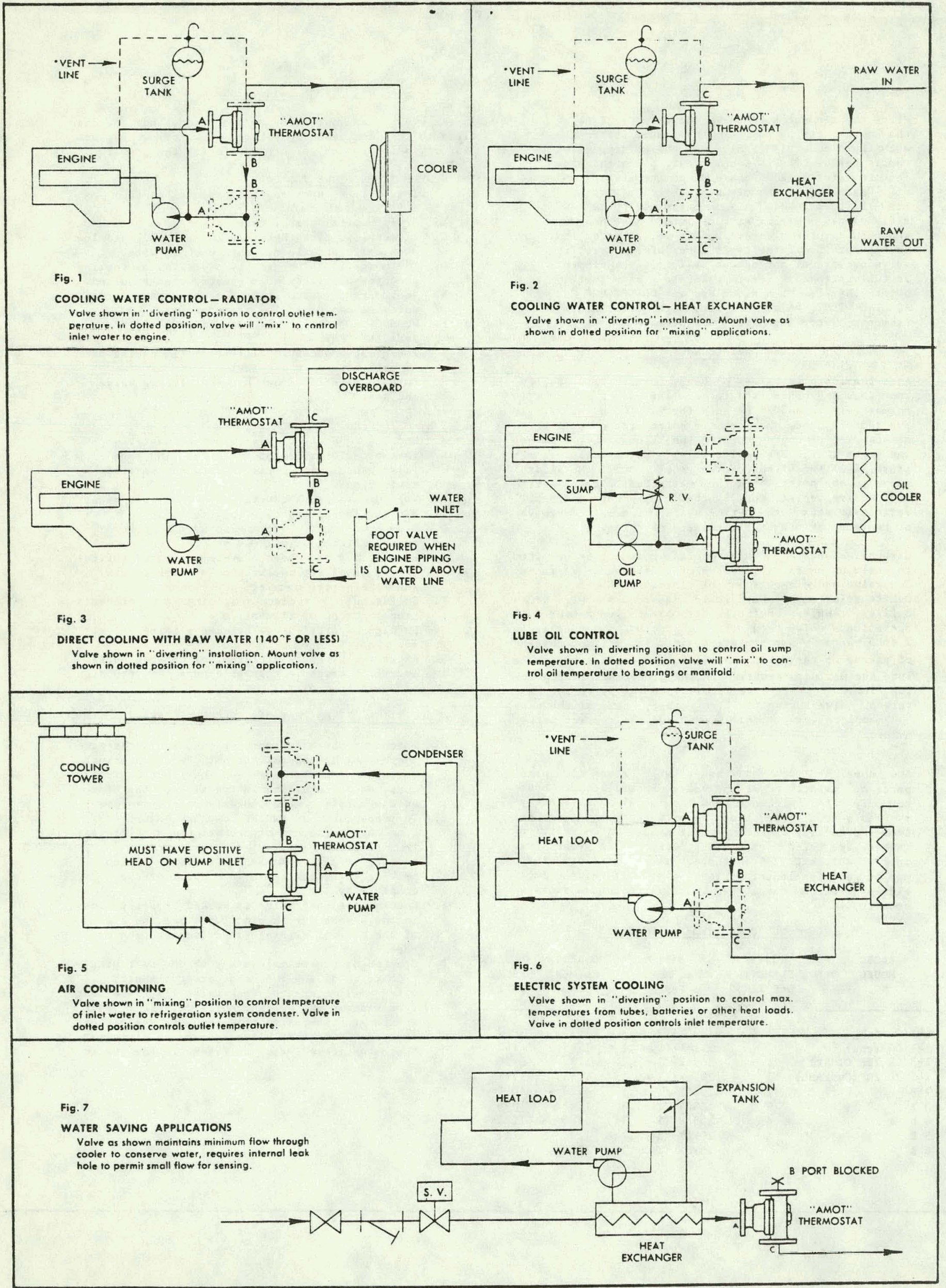


PARTS LIST

\section{MODELS $1 \frac{1}{2}$ A 2B 2BF}

(1)

\begin{tabular}{|c|c|c|c|c|}
\hline $\begin{array}{l}\text { REF. } \\
\text { NO. }\end{array}$ & DESCRIPTION & $\begin{array}{l}\text { QTY. } \\
\text { PER UNIT }\end{array}$ & $\begin{array}{c}1-1 / 2 A \\
\text { PART NOS. }\end{array}$ & $\begin{array}{l}2 B \& 2 B F \\
\text { PART NOS. }\end{array}$ \\
\hline 1 & HOUSING ASSY. & 1 & NOTF 4 & NOTE 4 \\
\hline 2 & UPPER HSG & 1 & NOTE 4 & NOTE 4 \\
\hline 3 & SEAT & 7 & NOT REQD. & 1092 \\
\hline 4 & SLEEVE & 1 & 3904 & $5 \overline{634}$ \\
\hline 5 & 0 RING SEAL & $i$ & 1919 & 1183 \\
\hline 7 & ELEMENT ASSY. & $i$ & $1126 \times \ldots$ & $1096 \times \ldots$ \\
\hline 8 & FLAT GASKET & T & 1067 & $\$$ \\
\hline $8 A$ & $O$ RING GASKET & 1 & 761 & 761 \\
\hline 9 & LOWER HSG. & 1 & NOTE 4 & NOTE 4 \\
\hline 10 & CAPSCREW & 4 & -46 & ti \\
\hline 11 & LOCKWASHER & 4 & $\neq \frac{2 \pi}{2}$ & $\neq \neq$ \\
\hline 172 & NUT & 4 & 棸 & $\Rightarrow$ \\
\hline
\end{tabular}

(1) ON MODEL $1-1 / 2 A$, THE SEAT IS NOT REPLACE$A B L E$.

(2) FLAT GASKET USED ON ALL OLDER SINGLE

ELEMENT VALVES HAVING SHORT MODEL NO.

(SUCH AS $1-1 / 2 A-170,2 B F-160$, ETC).

(3) O RING GASKET USED ON ALL CURRENT SINGLE

ELEMENT VALVES HAVING LONGER MODEL NO.

(SUCH AS $1-1 / 2 A O B-170-01,2 B F C-160-01$,

ETC.).

(4) ORDER HOUSINGS BY STATING THE MODEL NO.

AND MATERIAL REOUIRED.
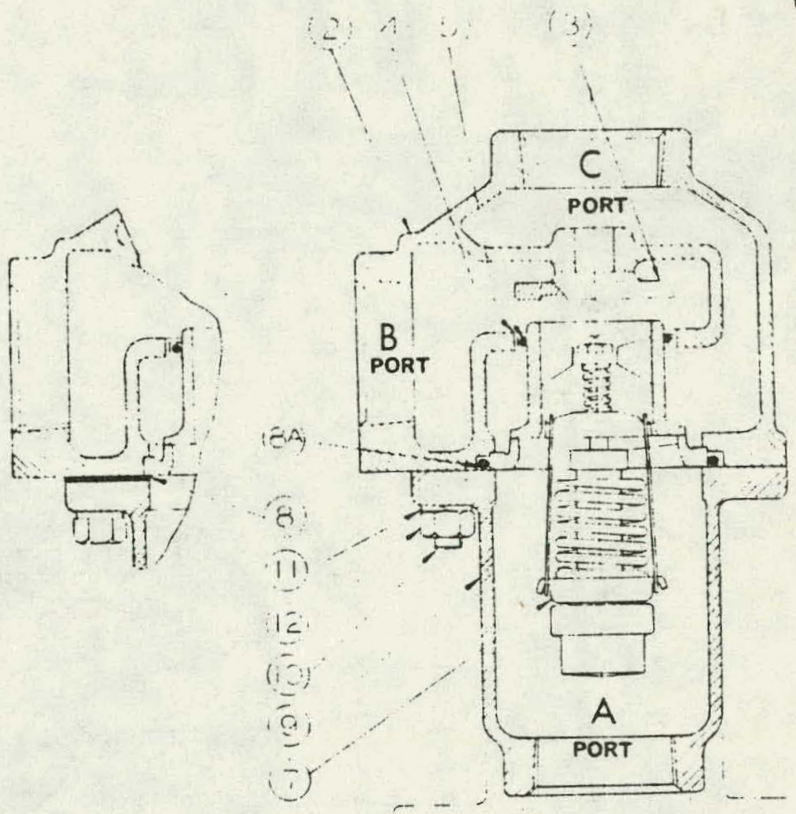

* oRder by REFERENCE nO., DFSCRIPTION AND

VAL Ve model nO. E.G. H10. CAPSCREW FOR 4B

VALVE, \#8, FLAT GASKET FOR 2R VALVE.

\section{PARTS LIST}

MODELS $2 \frac{1}{2}$ B 3 B 4 B 5 B $\quad 6 B$

\begin{tabular}{|c|c|c|c|c|c|c|c|}
\hline $\begin{array}{l}\text { REF. } \\
\text { NO. }\end{array}$ & DESCRIPTION & $\begin{array}{l}\text { QUANT I } \\
2-1 / 2 B\end{array}$ & $\frac{Y}{3 B}$ & $\frac{Q^{\prime} \cdot D}{4 B}$ & & $\underset{-W B}{U N}$ & $\begin{array}{c}\text { PART } \\
\text { NUMBERS }\end{array}$ \\
\hline 1 & HOUSING ASSY. & 1 & 1 & 1 & 1 & 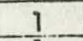 & NOTE 4 \\
\hline 2 & UPPER HSG. & $i$ & 1 & 1 & $\frac{1}{5}$ & $\frac{1}{0}$ & NOTE 4 \\
\hline 3 & SEAT & 2 & 2 & 4 & 6 & 9 & 1092 \\
\hline 4 & SLEEVE & 2 & 2 & 4 & 6 & 9 & 1182 \\
\hline 5 & $O$ RING SEAL & 2 & 2 & 4 & 6 & 9 & 1183 \\
\hline 6 & STUD & 0 & 0 & 2 & 2 & 4 & $\$$ \\
\hline 7 & ELEMENT ASSY. & 2 & 2 & 4 & 6 & 9 & $1096 x \ldots$ \\
\hline 8 & FLAT GASKET & 1 & 7 & 7 & 1 & 1 & \\
\hline 9 & DOWER HSG & $T$ & 1 & $T$ & 1 & $T$ & NOTE 4 \\
\hline 10 & CAPSCREW & $\frac{1}{4}$ & 4 & 6 & 6 & 8 & $1+*$ \\
\hline 17 & LOCKWASHER & 4 & 4 & 8 & 8 & 12 & $* \star$ \\
\hline 12 & NUT & 4 & 4 & 8 & 8 & 12 & $\hbar *$ \\
\hline
\end{tabular}

* order elements by basic monel no. ann TEMPERATURE. E.G. $1126 \times-150^{\circ}$. $1096 \times-180^{\circ}$.

* ORDER BY REFERENCE NO., DESCRIPTION AND VALVE MODEL NO. E.G. WO. CAPSCREW FOR 4R VALVE, 8 . FLAT GASKET FOR 3B VALVE.

(4) ORDER HOUSINGS BY STATING THE MODEL NO. AND MATERIAL REQUIRED.

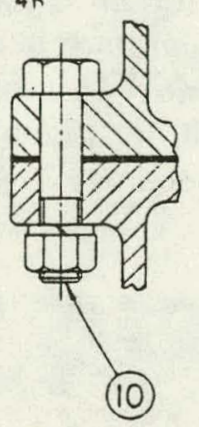

(2)

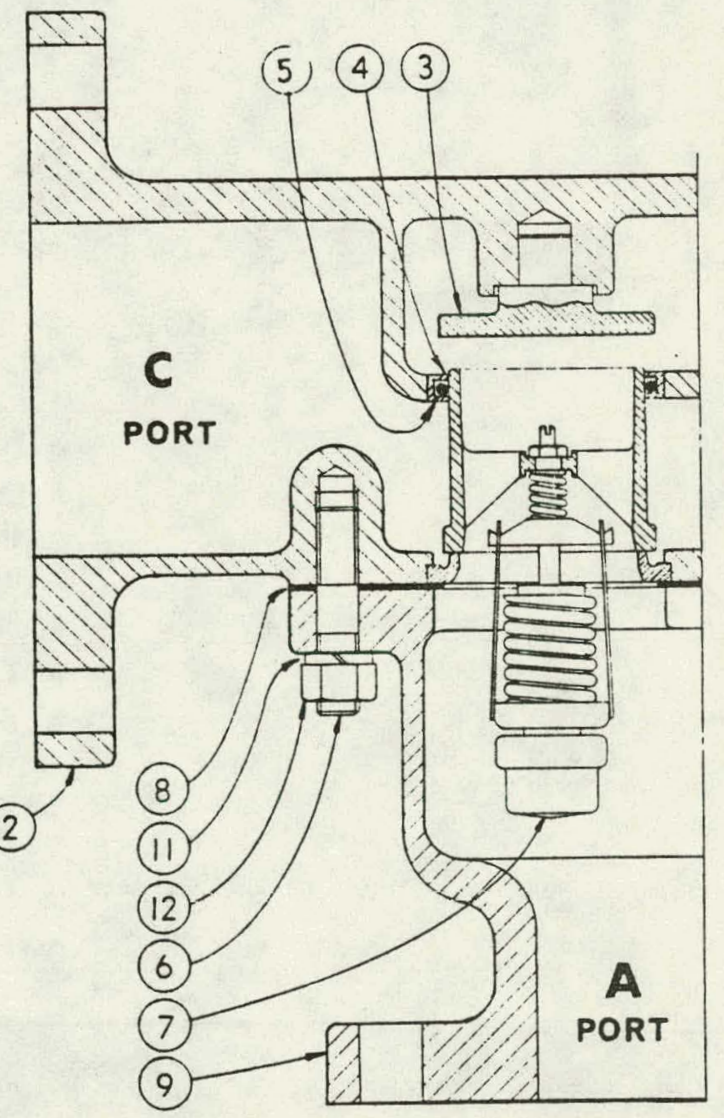




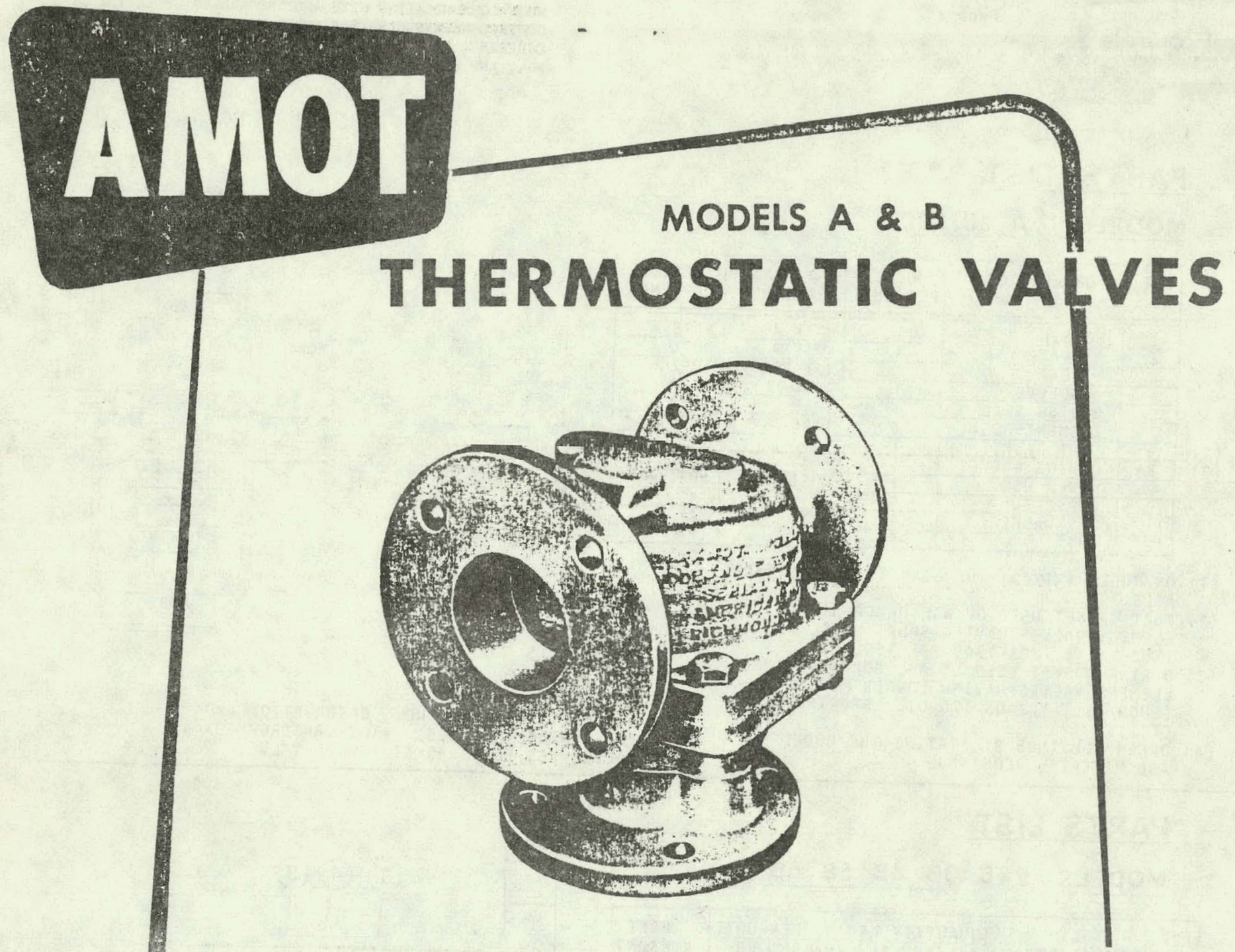

AMOT Thermostals offer the following advantages for automatic engine jacket water and lubricating oil temperature control:

- Large capacity combined with light weight.

- Excellent reliability even under extreme shocks and vibration.

- No external bulbs or tubing to break and cause trouble.

- Operating forces are very large and with no packing glands, there is no chance of valve ever sticking or becoming inoperative.

- Holds close regulation and is not sensitive to pressure.

- Temperature is fixed and cannot be tampered with.

- Because of its simple and rugged construction, practically no maintenance is ever required.

- Compact in size, and will operate in any position, which facilitates piping.

- Has positive 3-way action.

- Prices are competitive.

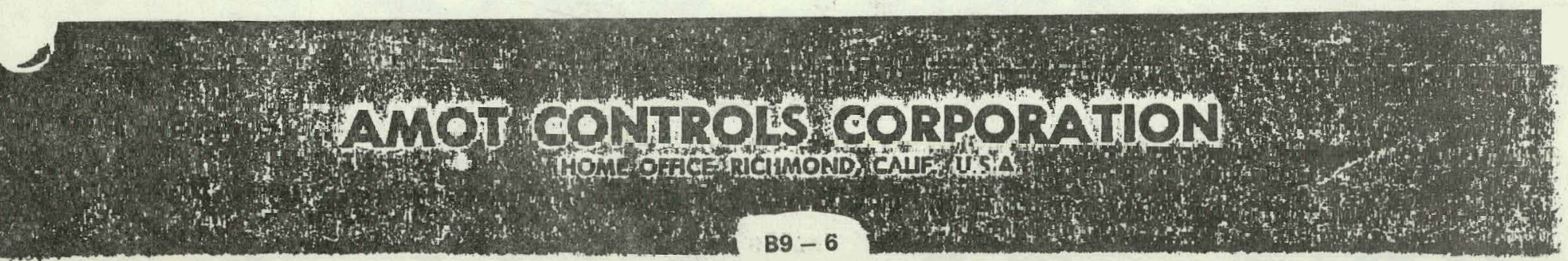




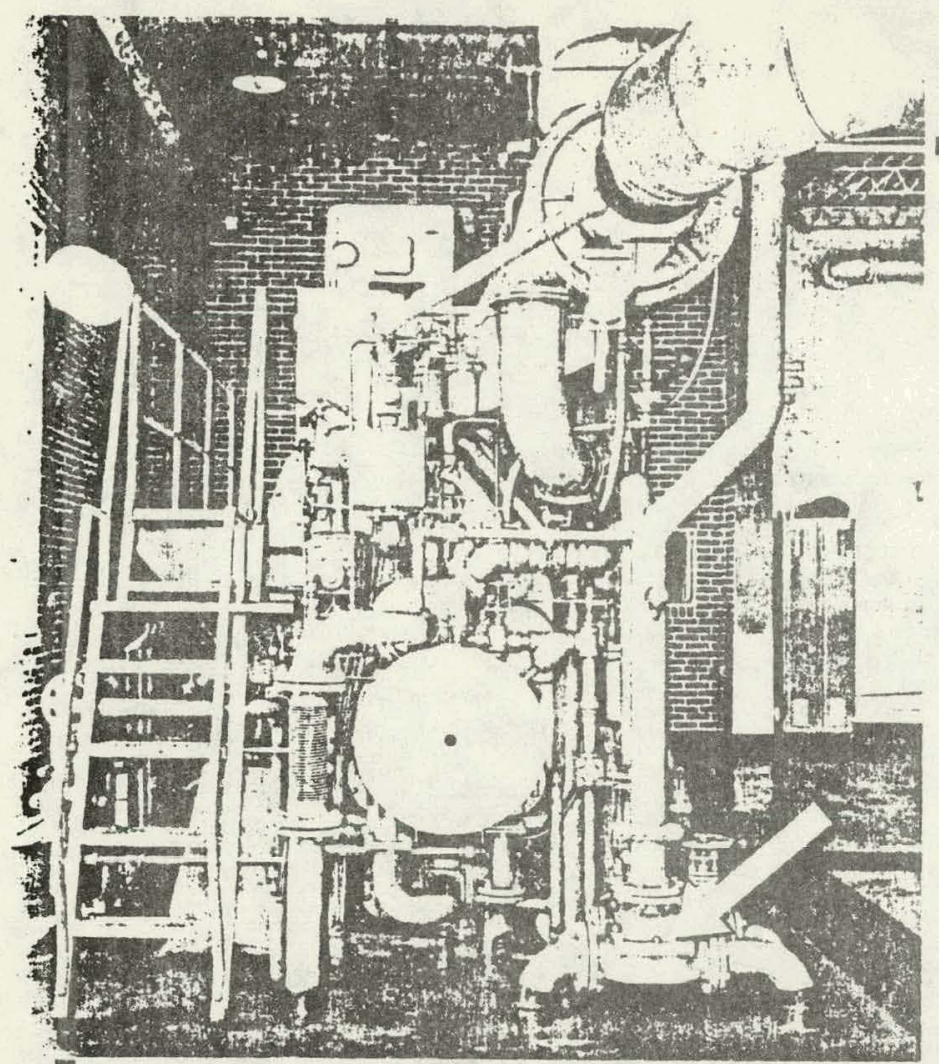

Fig. 1. Typical Amot thermostat installation on a 1200 hp dual fuel engine at Phillips Pipe Line Sharpe, Kansas station. Arrow shows position of a 4 " Amol thermostat in the engine water outlet line.

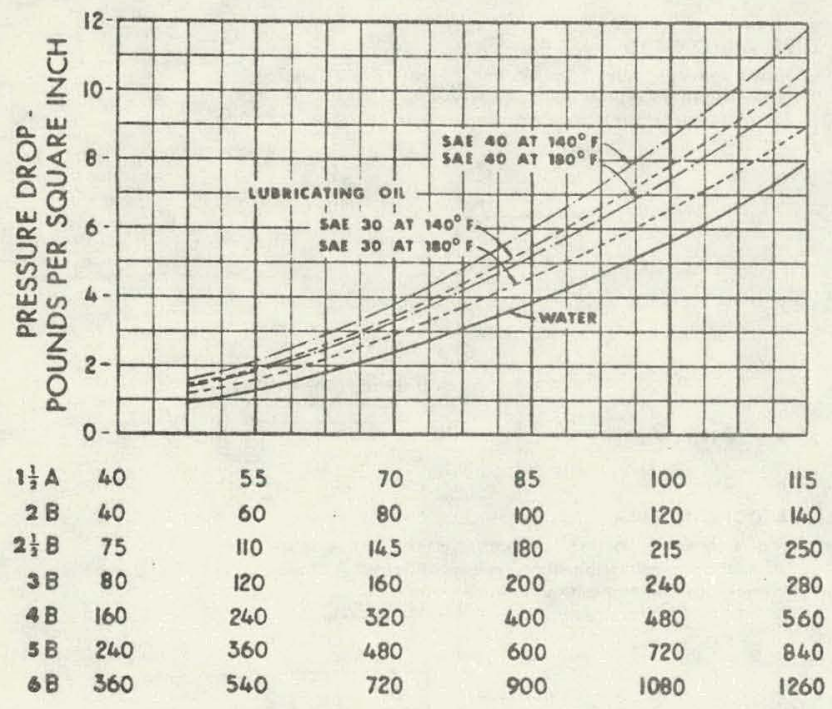

FLOW IN U.S. GALLONS PER MINUTE Recommended pressure drop is 2 to 7 psi.

Fig. 2. Pressure drop through Amot thermostats.

\section{GENERAL INFORMATION ON AMOT THERMOSTATS}

All AMOT thermostats are equipped with positive 3-way valve action in which the water or lubricating oil is positively made to flow in the direction required. On jacket water applications when the engine is started up and is cold, the AMOT thermostat causes all of the water to be positively by-passed back into the engine, thus providing the quickest warm-up period possible. After warm up, the correct amount of water is by-passed and automatically mixed with the cold water returning from the heat exchanger or other cooling device to produce the desired jacket water outlet temperature. If ever required, the AMOT thermostat will shut off positively on the by-pass line. The 3-way action of the AMOT thermostat allows constant volume of water through the pump and engine at all times with no pump restriction when the engine is cold.

No adjusiments or service are ever required on AMOT thermostats. Once installed an AMOT thermostat can be forgoften about. It is entirely sell-contained, and there are no external bulbs or lines to become damaged or broken. There are no packing glands to fighten and no parts to oil. The temperature is permanently sef af the foctory and requires no further adjustment. The operating temperature can be changed only by changing elements. Elements are easily replaced by unbolting the housing. Elements ore all interchangeable befween the 2" to 6" sizes and sizes larger than 2" contain multiple elements in one housing. This means that the same size AMOT element will fit any size AMOT thermostat housing from the 2" pipe size to the 6" pipe size model.

Because AMOT thermostats are set to a predetermined lemperature at the factory, costly errors due to mistakes of operating personnel are eliminated. After an AMOT thermostat has been installed, it is impossible for the operator to change the operating temperature and run the engine too cold unless the elements themselves are changed.

The femperature range of any AMOT thermostat ordered should follow the engine builder's recommendation. This information is usually ovailable from AMOT engineers who work closely with the engine manufacturers. A few general recommendations can be made, however. For direct salt water cooled marine engines the $120^{\circ} \mathrm{F}$. model should be used as this temperature is well below the point at which salt will deposit in the water passages of large diesel engines. Although it is saie to go somewhat higher than this temperature it is general practice to use the $120^{\circ} \mathrm{F}$. model for diesels and the $140^{\circ} \mathrm{F}$. model for smaller gasoline marine engines with direct salt water cooling. Special salt water thermostats are available at extra cost in the $120^{\circ} \mathrm{F}$. and $140^{\circ} \mathrm{F}$. models. For marine and stationary engines using heat exchangers, radiators, or some other type of fresh water system a jacket water outlet temperature of $160^{\circ} \mathrm{F}$. is fairly universal. In regard to size, the size thermostat corresponding to the water outlet pipe size on the engine is usually satisfactory. However, if waler flow information is available, the graph on figure 2 can be used to select the proper size. Pressure drop is usually limited to approximately 7 pounds per square inch.

Thermostat housings in the 2" flanged size and larger are furnished in either cast iron, ductile iron, or all bronze. The cast iron models are recommended for most applications because of their equal performance at a lower price. All bronze models are sometimes used on Navy installations when all bronze construttion is specified. The 2" size with screwed connections and smaller sizes are furnished in all bronze only. $1 / 2$ " to $11 / 4^{\prime \prime}$, and models larger than 6 " AMOT thermostats are also available 
and are described in other bulletins. Dimensions and weights of AMOT thermostats are shown on the last page of this bulletin.

\section{PIPING DIAGRAMS FOR AMOT THERMOSTATS}

The two most commonly used piping diagrams for jacket water control are shown in figures 4 and 5 . The AMOT thermostat will operate in any position and mounting should be made in accordance with convenience.

For lubricating oil temperature control, the AMOT thermostat is used directly in the lubricating oil line as shown in figure 6 . The oil will by-pass the heat exchanger when cold and will reach the desired operating temperature rapidly. When warm, the corred amount of oil will be circulated through the heat exchanger to maintain the desired remperature automatically. The system shown in fig. $6 \mathrm{~A}$ is similar to the standard jacket water temperature control system. In fig. 6B the AMOT thermostat is used as a mixing valve. Hot oil enters "B" and cold oil enters " $\mathrm{C}$ " and the oil is mixed and proportioned so as to emerge from " $\mathrm{A}$ " at the desired temperature. This system is sometimes used in connection with jacket water femperature control when it is desired to maintain a cestain temperature of the water entering the engine.

\section{FEATURES OF AMOT THERMOSTAT ELEMENTS}

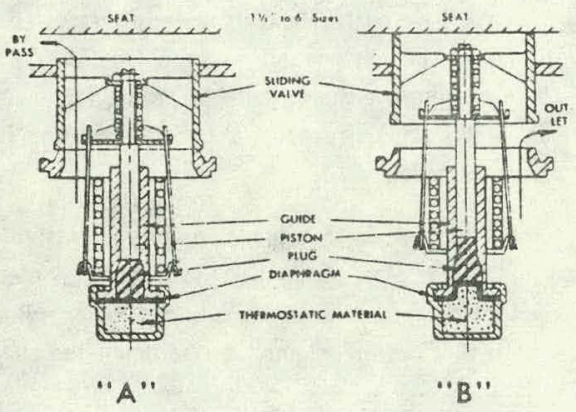

Fig. 3.

The power creating medium utilizes the expansion of a special thermustutic material which remains in o semi-solid form and which is highly sensitive to temperature changes.

The operating range is defermined by the chemical composition of the material. Expansion of the material created by a rise in temperature develops a pressure that is transmitfed directly to the piston producing a very large force which easily overcomes the refurn spring force of over 100 pounds. Construction is simple and rugged, yet the unit is very sensitive to changes in femperaiure. The element can stand extreme temperafures without damage or change in calibration. Changes in pressure do not affect the element and due to the valve construction surges in pressure do not tend to upset the stability of the thermostat.

" $A$ " in figure 3 shows the element with the operating piston in the cold position. The woter trovels out the by-pass as shown by the arrow.

" $\mathrm{B}$ " in figure 3 shows the piston moved up to the extended or worm position. The by-pass closes off as the sliding valve seats and the water travels out the outlet as shown by the arrow. In actual operation, the sliding valve is normally in about the mid-position. When the unit operates, the expansion of the thermostat material forces a moulded synthetic rubber plug into a reduced diameter in the piston guide, which multiplies the movement of the piston by an extruding action. The plug and diaphragm are of a synthetic composition.
Fig. 4 .

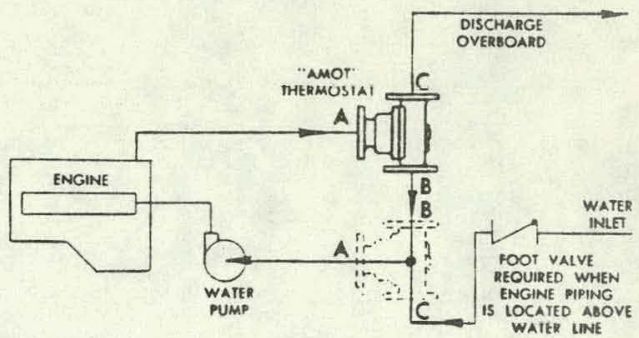

DIRECT COOLING WITH RAW WATER (140 F OR LESS)

Valve shown in "diverting" installation. Mount valve as.

shown in dotted position for "mixing" applications.

Fig. 5.

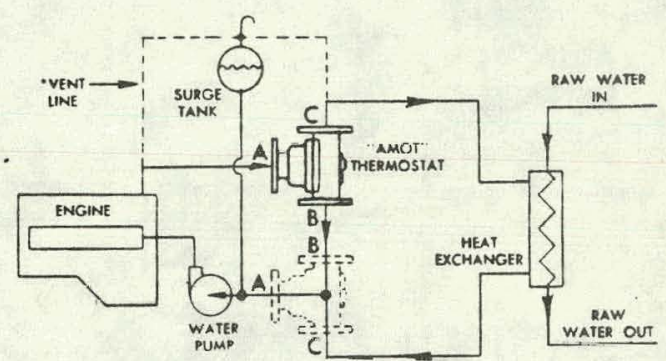

COOLING WATER CONTROL - HEAT EXCHANGER

Valve shown in "diverting installation. Mount valve as shown in dotted position for "mixing" applications.

Fig. 6 .

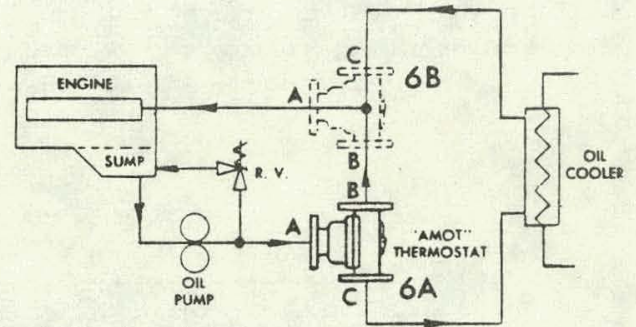

UUE OIL CONTROL

Valve shown in diverting position to control oil sump temperature. In dolled position volve will "mix" to control oil remperature lu beurings or manifold.

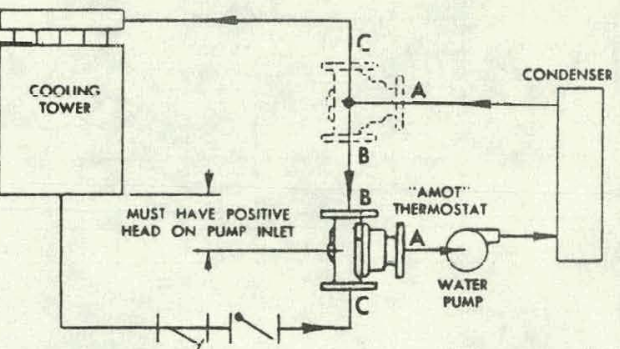

\section{AIR CONDITIONING}

Valve shown in "mixing" position to control temperature of inlet water to refrigeration system condenser. Valve in dotted position controls outlet temperature.

\section{Fig. 7.}

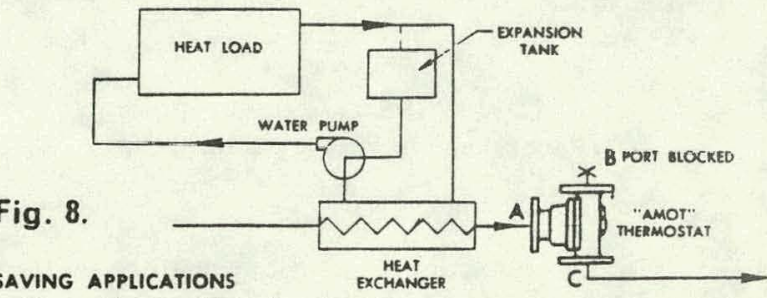

Valve as shown maintains minimum flow through

cooler to conserve water, requires internal leak hole to permit small flow for sensing. 


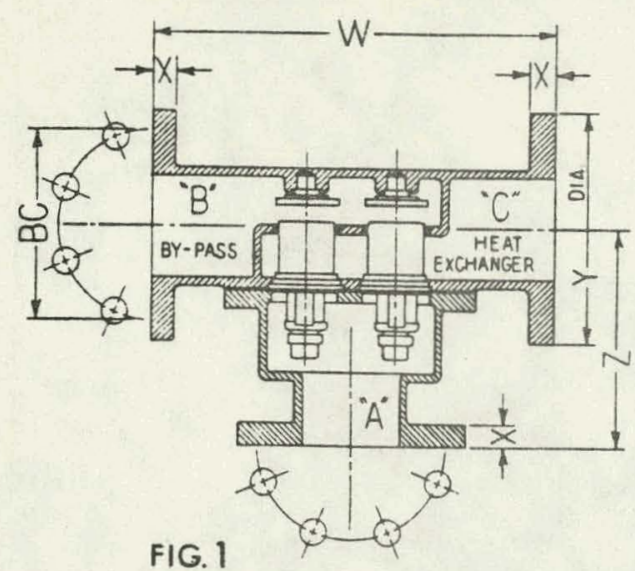

$2 \frac{1}{2}$ TO O" PIPE SIZE 125 PSI AMER STD, FLANGES

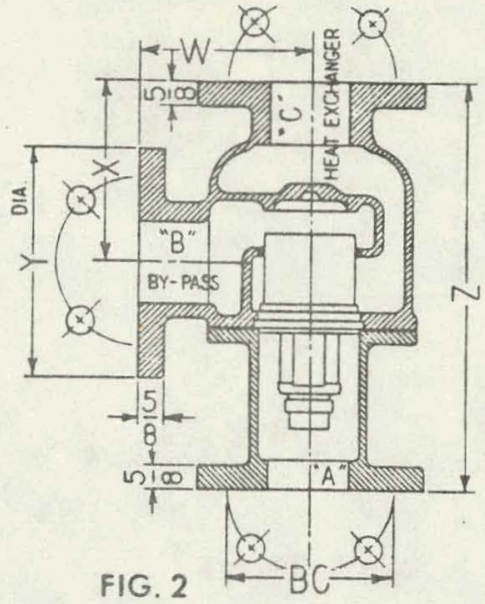

2"PIPE SIZE

125 PSI AMER. STD. FLANGE

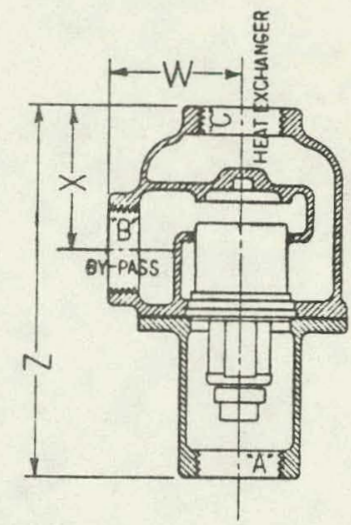

FIG. 3

I 2 " 82 2"PIPE SIZES SCREWED CONNECTIONS

\begin{tabular}{|c|c|c|c|c|c|c|c|c|c|c|c|c|c|c|}
\hline \multirow{2}{*}{$\begin{array}{l}\text { MODEL } \\
\text { NO. }\end{array}$} & \multirow{2}{*}{$\begin{array}{l}\text { PIPE } \\
\text { SIZE }\end{array}$} & \multirow{2}{*}{$\begin{array}{l}\text { FIG. } \\
\text { NO. }\end{array}$} & \multicolumn{4}{|c|}{ PRINCIPAL DIMENSIONS } & \multirow{2}{*}{\begin{tabular}{l|} 
MAX \\
WIDTH \\
OTHER \\
PLANE \\
\end{tabular}} & \multicolumn{3}{|c|}{ FLANGE DRILLING } & \multirow{2}{*}{$\begin{array}{l}\text { NO OF } \\
\text { ELE- } \\
\text { MENTS }\end{array}$} & \multicolumn{2}{|c|}{ SHIPPING WT. } & \multirow{2}{*}{$\begin{array}{l}\text { EXPORT } \\
\text { CUBIC } \\
\text { FEET }\end{array}$} \\
\hline & & & W & $x$ & $Y$ & $z$ & & $\begin{array}{l}\text { NO. OF } \\
\text { HOLES }\end{array}$ & $\begin{array}{l}\text { DIA. OF } \\
\text { HOLES }\end{array}$ & B.C. & & IRON & BRONZE & \\
\hline $1 \frac{1}{2} \mathrm{~A}$ & $1 \frac{1 "}{2}$ & 3 & $3 \frac{1}{8}$ & $2 \frac{13}{16}$ & - & $8 \frac{5}{6}$ & $4 \frac{7}{8}$ & - & - & - & 1 & 18 & 20 & .4 \\
\hline $2 \mathrm{~B}$ & 2 & 3 & $3 \frac{2}{16}$ & $3 \frac{13}{16}$ & - & $9 \frac{3}{4}$ & $5 \frac{1}{2}$ & - & - & - & 1 & 22 & 25 & .4 \\
\hline $2 \mathrm{BF}$ & 2 & 2 & $4 \frac{7}{16}$ & $4 \frac{3}{4}$ & 6 & $10 \frac{5}{8}$ & 6 & 4 & $\frac{3}{4}$ & $4 \frac{3}{4}$ & 1 & 35 & 40 & .4 \\
\hline $2 \frac{1}{2} B$ & $2 \frac{11}{2}$ & 1 & 10 & $\frac{11}{16}$ & 7 & $6 \frac{1}{2}$ & $8 \frac{1}{4}$ & 4 & $\frac{3}{4}$ & $5 \frac{1}{2}$ & 2 & 55 & 75 & 1.1 \\
\hline $3 \mathrm{~B}$ & 3 & 1 & $10 \frac{1}{2}$ & $\frac{3}{4}$ & $7 \frac{1}{2}$ & $6 \frac{3}{4}$ & $8 \frac{1}{4}$ & 4 & $\frac{3}{4}$ & 6 & 2 & 60 & 80 & 1.1 \\
\hline 4B & $4^{\prime \prime}$ & 1 & $15 \frac{7}{8}$ & $\frac{15}{16}$ & 9 & $8 \frac{9}{16}$ & $12 \frac{1}{4}$ & 8 & $\frac{3}{4}$ & $7 \frac{1}{2}$ & 4 & 135 & 150 & 24 \\
\hline $5 B$ & $5^{\prime \prime}$ & 1 & $19 \frac{1}{4}$ & $\frac{15}{16}$ & 10 & $9 \frac{1}{2}$ & 14 & 8 & $\frac{7}{8}$ & $8 \frac{1}{2}$ & 6 & 200 & 240 & 3.5 \\
\hline $6 B$ & $6^{\prime \prime}$ & 1 & $19 \frac{1}{4}$ & 1 & 11 & 10 & 19 & 8 & $\frac{7}{8}$ & $9 \frac{1}{2}$ & 9 & 270 & 300 & 5.3 \\
\hline
\end{tabular}

BASIC MODELS $1 \frac{1}{2} A, 2 B$ AND 2BF ARE AVAILABLE IN CAST IRON OR ALL BRONZE. BASIC MODELS 21/2B THROUGH 6B ARE AVAILABLE IN CAST IRON, DUCTILE IRON OR ALL BRONZE. CAST IRON AND DUCTILE IRON VALVES ARE BRONZE FITTED. SPECIFY HOUSING MATERIAL.

VALVES MAY BE ORDERED BY SPECIFYING THE BASIC MODEL NO., THE HOUSING MATERIAL DESIRED THE NOMINAL TEMPERATURE DESIRED, AND ANY SPECIAL CONDITIONS THE VALVE MUST WITH. STAND. AMOT HAS ADOPTED THE POLICY OF ADDING TO THE COMPLETE MODEL NUMBER A LETTER DESIGNATING ANY SPECIAL HOUSING FEATURES, ANOTHER LETTER TO DESIGNATE HOUSING MATERIAL (C, B, D OR A, ETC.) AND A CODE NUMBER AFTER THE NOMINAL TEMPERATURE TO INDICATE TYPE OF ELEMENT. THUS A VALVE ORDËRED AS A $21 / 2 B-160$ CAST IRON BRONZE FITTED, WILL BE STAMPED ON THE NAMEPLATE AS A 21/2BOC-160-01. THE O MEANS STANDARD CONSTRUCTION, C IS CAST IRON, THE 01 MEANS STANDARD 1096 X-TYPE ELEMENT, THE 160 MEANS SET TO MAINTAIN A NOMINAL $160^{\circ} \mathrm{F}$ FLUID TEMPERATURE.

AMOT THERMOSTATIC VALVES ARE TEMPERATURE RATED FOR THE EXPECTED NOMINAL OPERATINC TEMPERATURE IN JACKET WATER SERVICE. ON LUBRICATING OIL APPLICATIONS THE OPERATING TEMPERATURE MAY BE SLIGHTLY ABOVE THE NOMINAL RATING, DEPENDING ON THE TYPE OF OIL, FLOW RATE, AND OTHER CONDITIONS OF THE SYSTEM.

STANDARD STOCK TEMPERATURE SETTINGS AVAILABLE FOR A AND B SERIES VALVES ARE 75, 90,105 $120,130,140,150,155,160,165,170,180$, AND $195^{\circ} \mathrm{F}$. SOME OTHER TEMPERATURE SETTINGS AVAIL ABLE ON SPECIAL ORDER ON LIMITED STOCK BASIS. BASIC MODEL $11 / 2 A$ REQUIRES ONE $1126 \times-(T E M P$.) TYPE THERMOSTATIC ELEMENT. BASIC MODEL B REQUIRES THE $1096 \times$-(TEMP.) TYPE ELEMENT WHICH IS INTERCHANGEABLE ON 2" THROUGH 6" SIZES.

2" TO 6" TYPE B VALVES ARE AVAILABLE WITH NAVY FLANGE DRILLING, AND MANUAL OVERRIDE IF DESIRED.
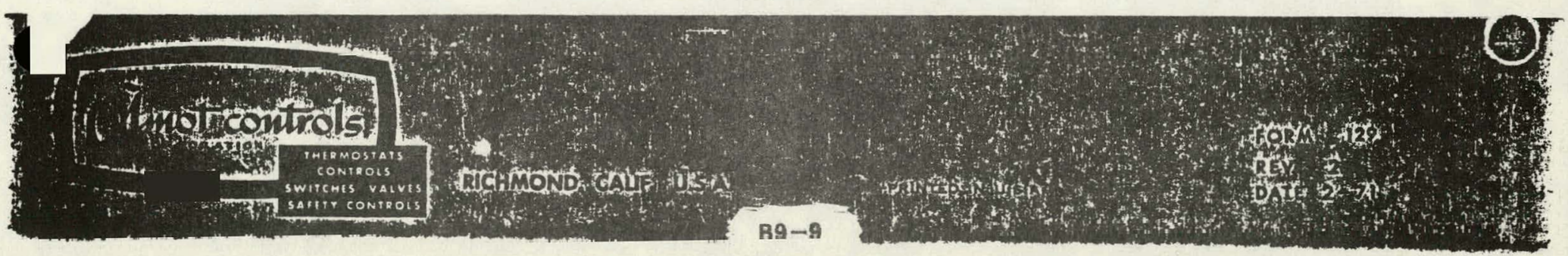


\section{Honeywell}

THE R7412 DIFFERENTIAL TEMPERATURE CONTROLLER PROVIDES AUTOMATIC CONTROL OF CIRCULATING PUMPS, VALVES, DAMPERS, MOTORS, AND OTHER ACCESSURIES USED IN SOLAR ENERGY SYSTEMS.

- All models contain a solid state differential temperature controller.

$\square R 7412 B$ includes freeze protection.

- R7412C includes freeze protection and an auxiliary relay driver.

D R7412D includes overtemperature prutection.

- R7412E includes overtemperature protection and an auxiliury relay driver.

R7412F includes frceze and uvertempcrature protectinn (field adjustable), and an auxiliary relay driver.

- Plug-in resistors permit changing on and off temperature differential and adapt. ing $\mathrm{R} 7412$ for single function temperature control.

D R7412B-E overtemperature and freeze protection set points are selectable for factory setting, but are not field adjustable.

口 Uses one or two C773 Temperature Sensors.

R.L.

REV. $10.77(.07)$ 
$\left[\begin{array}{c}0 \\ i\end{array}\right.$

THE SPECIFICATIONS GIVEN IN THIS PUBLICATION DO NOT INCLUDE NORMAL MANUFACTURING TOLERANCES THEREFORE, THIS UNIT MAY NOT MATCH THE LISTED SPECIFICATIONS EXACTLY. ALSO, THISPRODUCT IS TESTED AND CALIBRATED UNDER CLOSELY CONTROLLED CONDITIONS, AND SOME MINOR DIFFERENCES IN PERFOR. MANCE CAN BE EXPECTED IF THOSE CONDITIONS ARE CHANGED.

\section{TRADELINE MODELS}

TRADELINE MODELS are selected and packaged for ease of stocking, ease of handling, and maximum replacement value. TRADELINE specifications are the same as those of standard models except as noted below.

TRADELINE MODEL AVAILABLE:

R7412F Differential Temperature Controller.
TRADELINE FEATURES:

- Includes freeze and overtemperature protection and an auxiliary relay driver.

- TRADELINE Pack with cross reference label.

STANDARO MODELS

\begin{tabular}{|c|c|c|c|c|}
\hline MODEL & $\begin{array}{l}\text { DIFF. TEMP. } \\
\text { CONTROL }\end{array}$ & $\begin{array}{c}\text { FREEZE } \\
\text { PROTECTION }\end{array}$ & $\begin{array}{c}\text { OVERTEMPERATURE } \\
\text { PROTECTION }\end{array}$ & $\begin{array}{c}\text { AUXILIARY RELAY } \\
\text { DRIVER }\end{array}$ \\
\hline$R 7412 \mathrm{~A}$ & $\mathrm{Yes}^{\mathrm{a}}$ & & & \\
\hline R7412B & Yesa & $Y_{e s}{ }^{a d}$ & & \\
\hline $\mathrm{R} 7412 \mathrm{C}$ & $Y_{e s}^{a}$ & $Y_{e s}{ }^{c d}$ & & $Y_{e j} \mathrm{C}$ \\
\hline$R 7412 \mathrm{D}$ & $Y_{e s}{ }^{a}$ & & Yes bd & \\
\hline$R 7412 E$ & $Y_{e s}{ }^{a}$ & & Yescd & - Yes ${ }^{\mathrm{C}}$ \\
\hline$R 7412 \mathrm{~F}$ & $Y_{e s}{ }^{a}$ & $Y_{e s}{ }^{\text {ac }}$ & Yes bc & $\mathrm{Yes}^{\mathrm{C}}$ \\
\hline
\end{tabular}

${ }^{a}$ Internal relay energizes. ${ }^{b}$ Internal relay de-energizes. ${ }^{c}$ Auxiliary relay energizes. OProtection setpoint is factory fixed.

TEMPERATURE SETTING RANGES:

Operating Range (as defined by the temperature of the low temperature sensor) -0 to plus $210 \mathrm{~F}$ [minus 18 to plus $99 \mathrm{C}$ ].

Differential Temperature Controller-Adjustable $O N$ and $O F F$ differentials from minus 10 to plus $40 \mathrm{~F}$ (minus 5.6 to plus $22.2 \mathrm{C}$ ). Factory. set at $18 \mathrm{~F}[10 \mathrm{C}]$ temperature difference $O N$ and $3 \mathrm{~F}[1.7 \mathrm{C}]$ temperature difference OFF. Plug in resistors vary settings (see Table 1, page 7).

Freeze Protection -

Field adjustable (R7412F only) in $5 \mathrm{~F}$ [ $3.2 \mathrm{C}$ ] increments frnm 140 to $190 \mathrm{~F}$ [57 to $89 \mathrm{C}$ ].
R7412B-E set points may be specified in $5 \mathrm{~F}$ [ $3.2 \mathrm{C}$ ] increments from 140 to $190 \mathrm{~F}$ [57 to $89 \mathrm{C}$ ], but they are factory fixed.

Overtemperature differential-10 F [5.5 C].

Freeze differential-3 F [1.7 C].

Overtemperature-

Field adjustable settings (R7412F only) at 37,42 or $47 \mathrm{~F}[3,6$, or $8 \mathrm{C}]$.

R7412B-E set points may be specified at 37,42 , or $47 \mathrm{~F}[3,6$, or $8 \mathrm{C}]$, but they are factory fixed

Factory-set at $140 \mathrm{~F}[66 \mathrm{C}]$.

\section{ORDERING INFORMATION}

WHEN PURCHASING REPLACEMENT AND MODERNIZATION PRODUCTS FROM YOUR TRADELINE WHOLESALER OR YOUR DISTRIBUTOR, REFER TO THE TRADELINE CATALOG OR PRICE SHEETS FOR COMPLETE ORDERING NUMBER, OR SPECIFY-

1. Differential Temperature Controller order number.

2. Temperature Sensor order number (two required).

IF YOU HAVE ADDITIONAL QUESTIONS, NEED FURTHER INFORMATION, OR WOULD LIKE TO COMMENT ON OUR PRODUCTS OR SERVICES, PLEASE WRITE OR PHONE:

1. YOUR LOCAL HONEYWELL RESIDENTIAL DIVISION SALES OFFICE (CHECK WHITE PAGES OF PHONE DIRECTORY).

2. RESIDENTIAL DIVISION CUSTOMER SERVICE HONE YWELL INC., 1885 DOUGLAS DRIVE NORTH MINNEAPOLIS, MINNESOTA 55422 (612) $542-7500$

IIN CANADA-HONEYWELL CONTROLS LIMITED, 740 ELLESMERE ROAD, SCARBOROUGH, ONTARIO MIP 2V9) INTERNATIONAL SALES AND SERVICE OFFICES IN ALL PRINCIPAL CITIES OF THE WORLD.
3. Immersion Well order number.

4. Accessories, if desired.

5. Optional specifications, if desired.

(continued on page 3) 

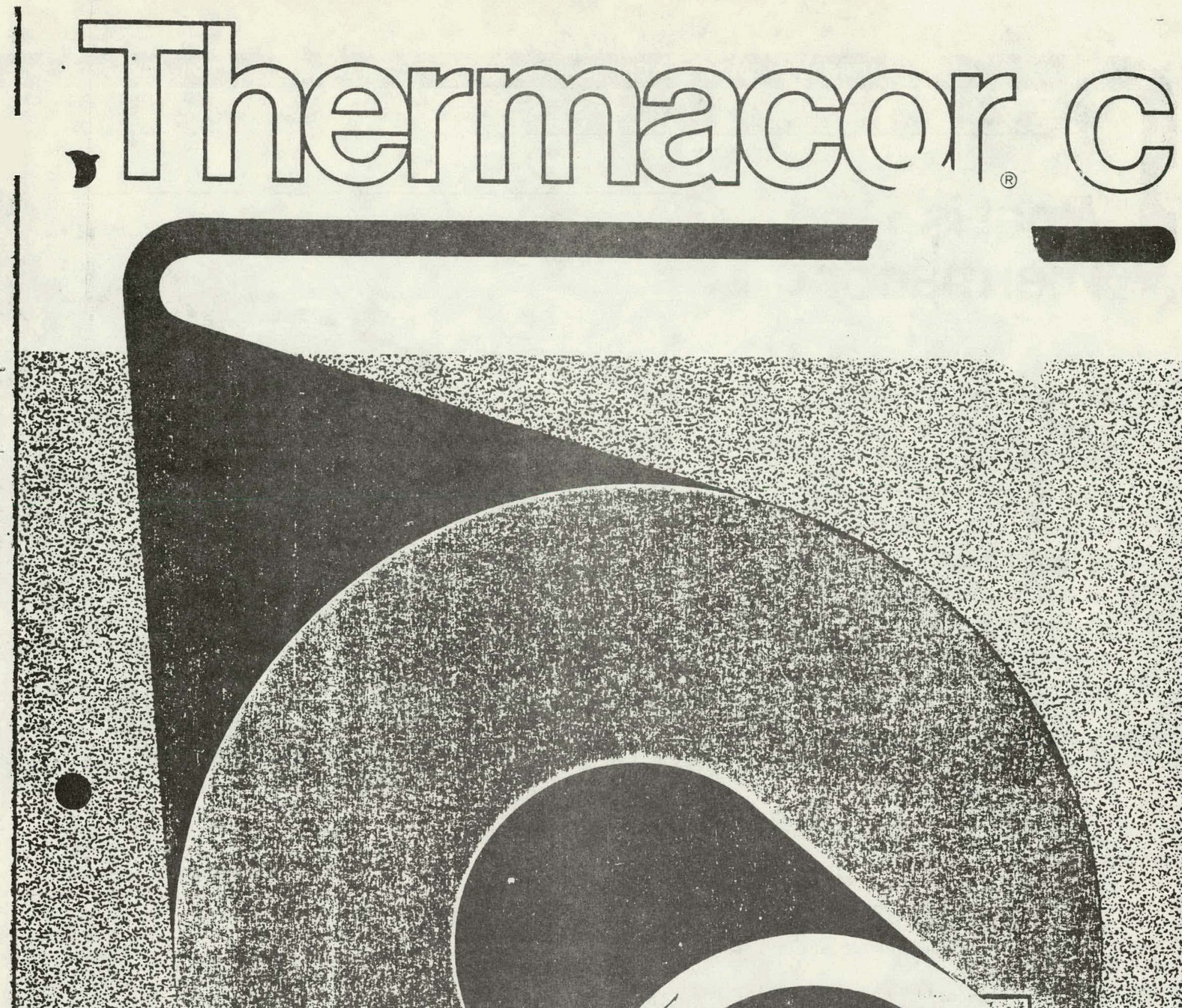

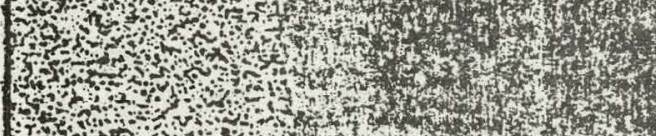

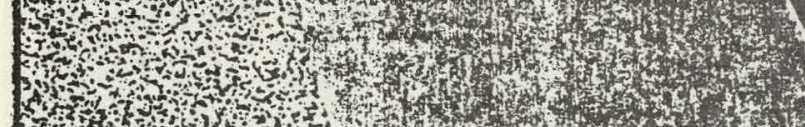

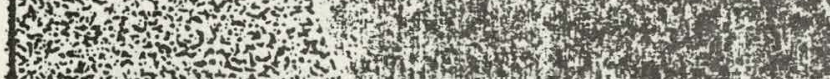

m.

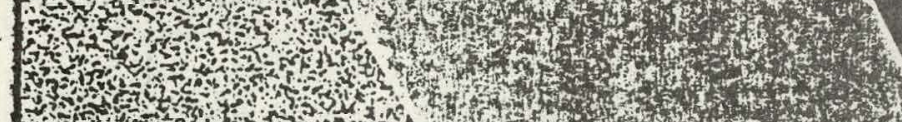

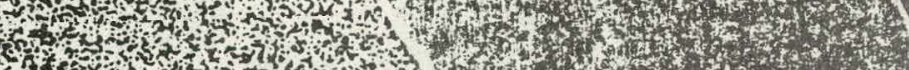

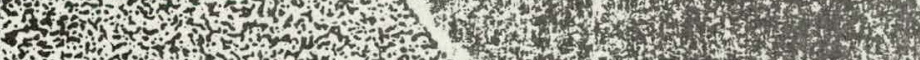

6.

(3.

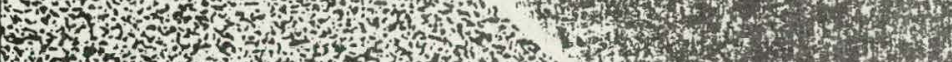

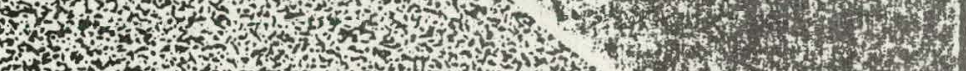

Shy

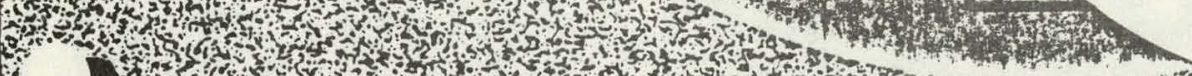

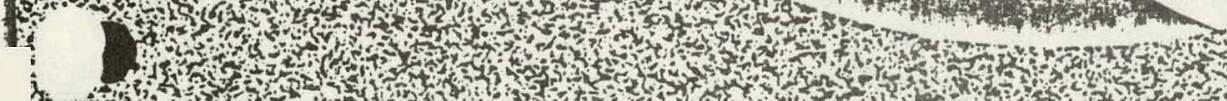

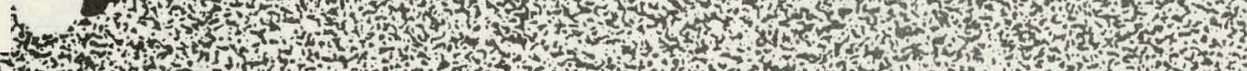

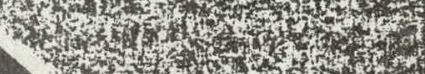

. 


\section{An exclusive coating which insulates and controls corrosion on nearly any type or size of pipe. It is $95 \%$ installed before it leaves the plant. And it is unequaled in creating dramatic savings and quality improvements in a host of applications.}

Thermacor Process Inc. of Fort Worth, Texas, has created Thermacor Type C - a revolutionary and exclusive patented pipe coating for thermal insulation and corrosion control using a urethane foam which is molded to the pipe in thicknesses of one or more inches. The coated pipe is then primed and wrapped with a polyvinyl chloride-butyl rubber laminate tape.

\footnotetext{
4 The Thermacor " $C$ " coating bonds exceptionally and equally well to all types of pipe including steel, iron, copper, plastic, galvanized, aluminum, and asbestos cement. It can be used on pipe with diameters extending from $1 / 2$ to 48 inches. The processing length of the pipe can be either $20 / 21$-foot standard lengths or 40 -foot random lengths. Pipe to be coated may be furnished through Thermacor Process Inc. or by the individual company before coating application. Thermacor " $C$ " is produced by a patented process whereby the pipe actually leaves the factory with $95 \%$ of the insulation already installed. Six inches at both ends of the coated sections are left bare for final on-site field joining and insulating. Special easy-to-use kits and molds are available for uniform insulation of joints, tees and elbows.
}

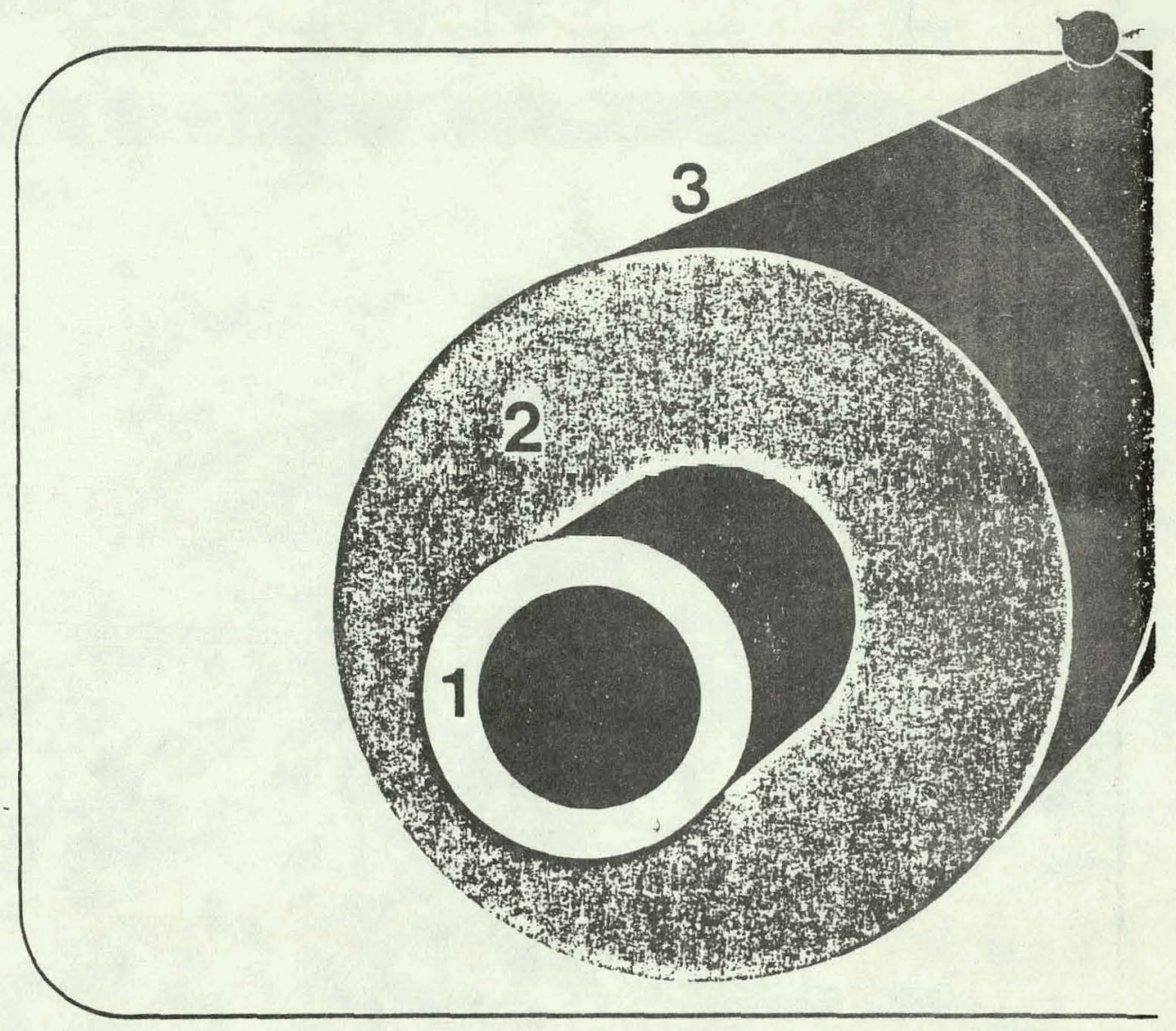

2 The inner layer of bonded-to-pipe urethane cells forms an anti-corrosive and moisture proof seal Even with a break in the outer layer, moisture will not penetrate. This layer also prevents any condensation that might result from temperature differential between the pipe and the environment. The outer layer - or skin - of high density urethane provides a protective barrier for the thick middle layer of closed-cell urethane... scientifically proven and well documented to be the best insulating material available.

3 The outer wrapping of polyvinyl chloride-butyl rubber laminate tape (which is available in black or white) applied over the urethane skin creates an extremely tough extra barrier to rocks, blows, earth shifts, etc. The tape's effectiveness as a vapor transmission and a di-electric barrier is a scientific fact. This combination of materials forming the Thermacor " $\mathrm{C}$ " coating, having a resistance of 30 to $48 \mathrm{PSI}$, provides a quality product that is creating major economic breakthroughs in numerous applications.

Thermacor " $\mathrm{C}$ " processed pipe is as near temperature proof as is possible to achieve with present technology against temperatures of between $-325^{\circ}$ and $+275^{\circ}$ Fahrenheit.

Special field installation kits containing the proper proportions of urethane components are available for connecting joints and fittings uniformly with the factory-applied coating ... leaving absolutely no cracks or voids. Also available are portable and reusable molds of various sizes. Installation of an on-site, thoroughly bonded connection is simple and accomplished in minutes.

Thermacor Process Inc., with the introduction of Thermacor " $\mathrm{C}$ ", has accomplished a major advance in the insulation of above and below ground pipe systems ... in both initial cost and long-term results. Thermacor has 


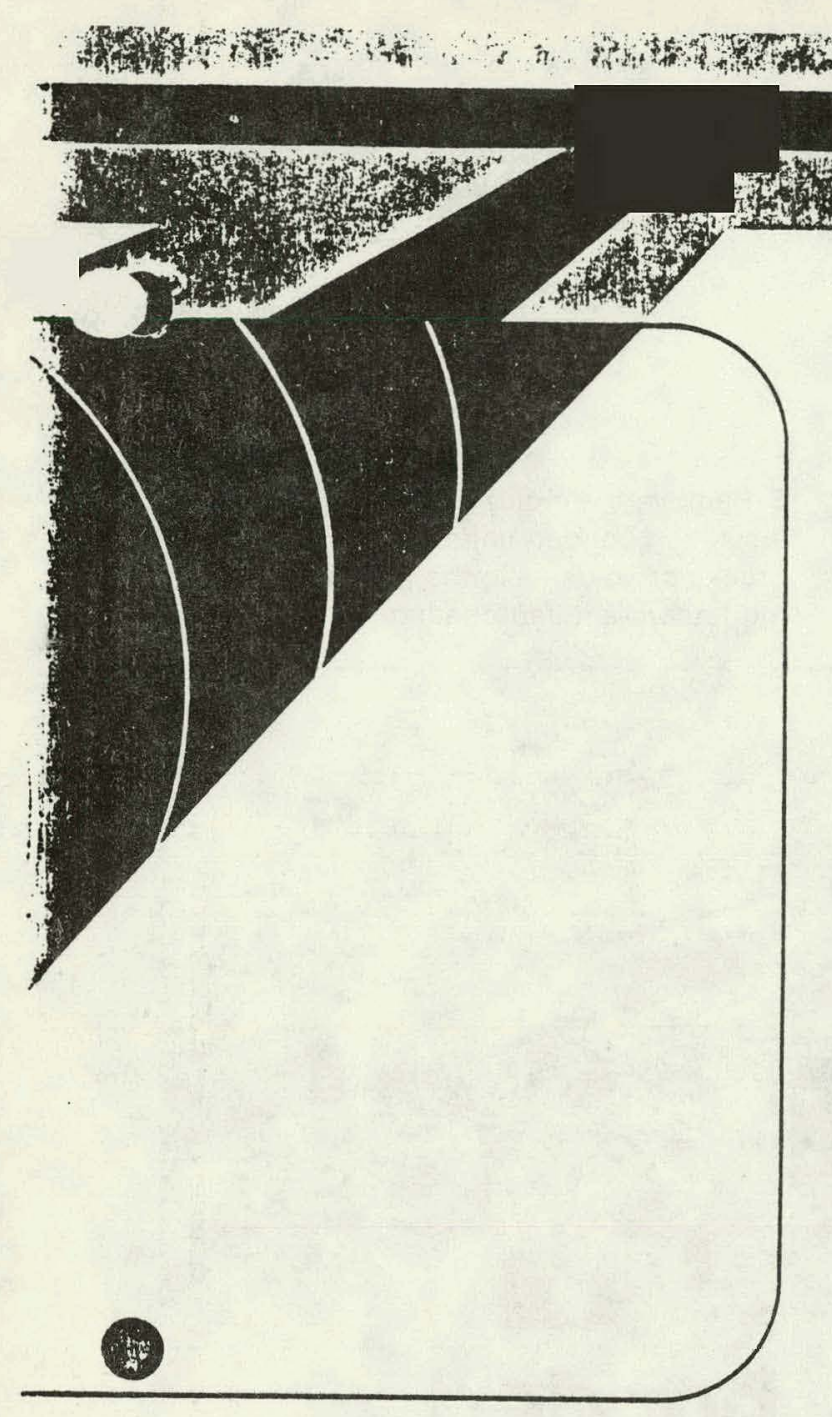

1 Thermacor Type $C$ coating is simple to install in the field. A sharp knife will smoothly cut through the urethane and the tape, if needed.
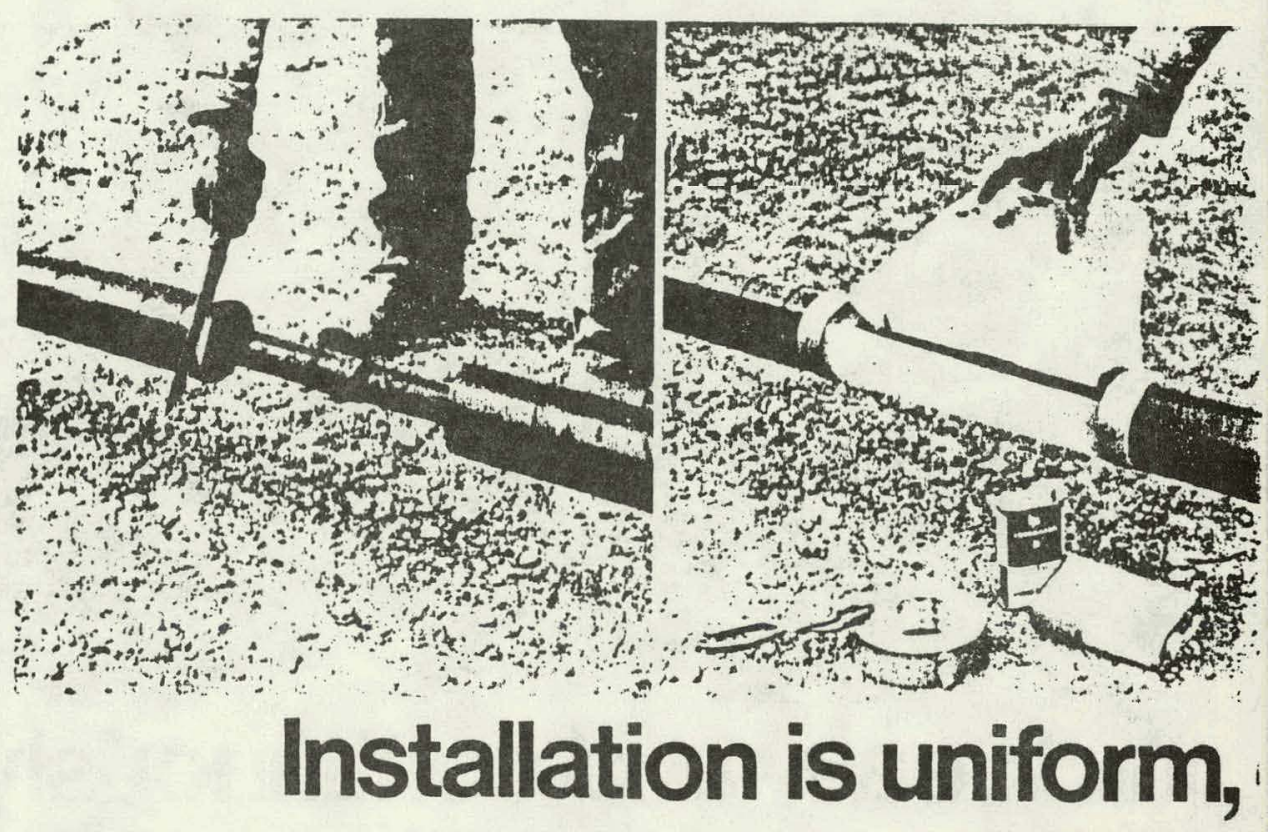

developed the maximum physical properties inherent in a specific formula of raw urethane foam and has adapted the process to more efficient, automated production techniques. It provides lower purchase cost, lower on-site field installation cost, ease of handling, and superior physical properties.

And, it has produced quality improvements in a host of industries and applications. By eliminating thermal conduits and expensive thick insulation, Thermacor " $\mathrm{C}$ " is ideal for underground low pressure steam, hot and chilled water, and domestic hot water distribution piping systems. It also is applicable to multi-building complexes, total energy systems and other large project piping ... natural gas piping... refinery piping... cryogenic piping ... low gravity or high paraffinic and crude transporting.

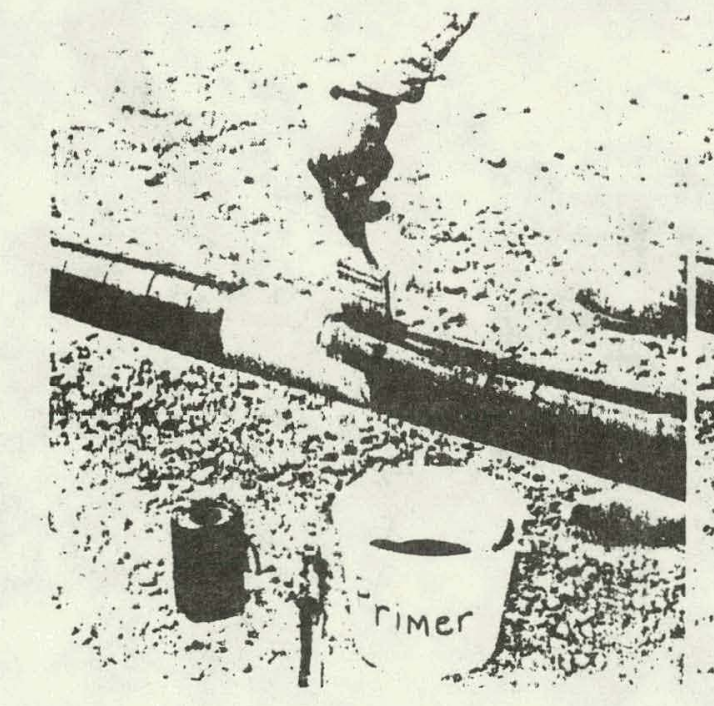

6 Apply a primer to the entire bare surface. An ordinary paint brush is sufficient to do the job.
2 On a straight coupling, merely tape the reusable mold securely onto the coated sections, as illustrated.

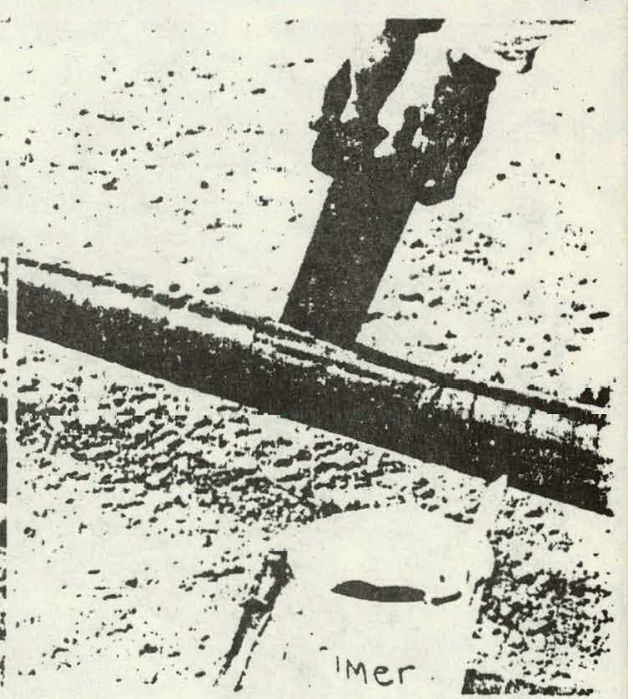

7 Wrap the primed area with the polyvinyl chloride-butyl rubber laminate tape to the primed surface. The field coating now is complete.

Thermacor " $C$ " makes new things possible through an economical premium product. 
iter the mold is in place, using the recommended kit of urethane components, pour the contents of Component $B$ into the container of Component A. Shake vigorously for at least 10 seconds. Hold the flap of the mold open then pour the contents evenly into the mold.

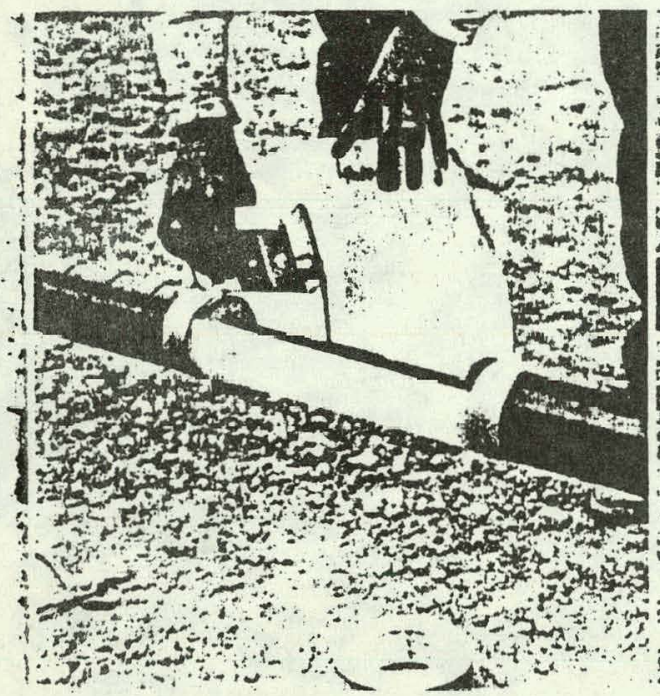

4 Tape the flap securely in place.

The material will then begin to foam. Allow approximately 20 minutes for the curing process. After curing, the urethane releases itself from the mold.
5 Remove the mold. You now have an insulation bonded uniformly - without cracks or voids - to the pipe and to the factory-applied coating.

\section{juick, easy and continuously bonded}

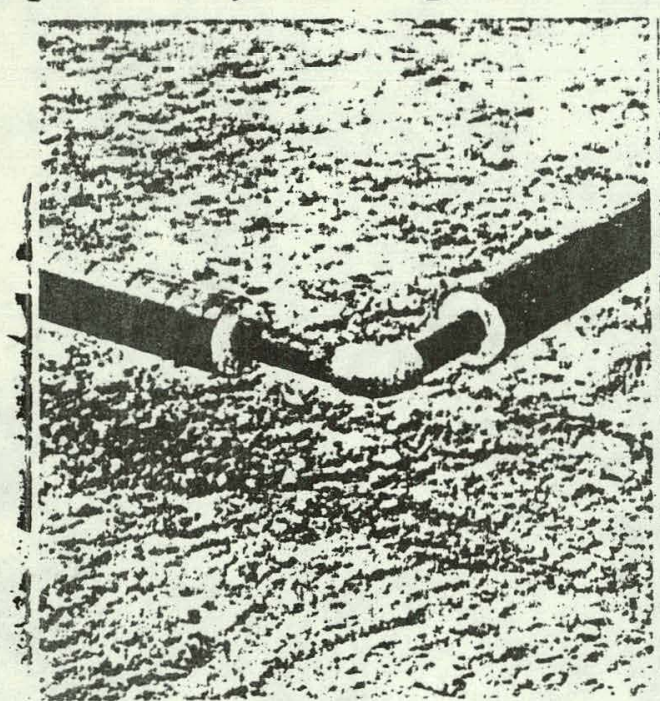

8 Elbow joints or tee connections are just as easy to complete using the Thermacor method.
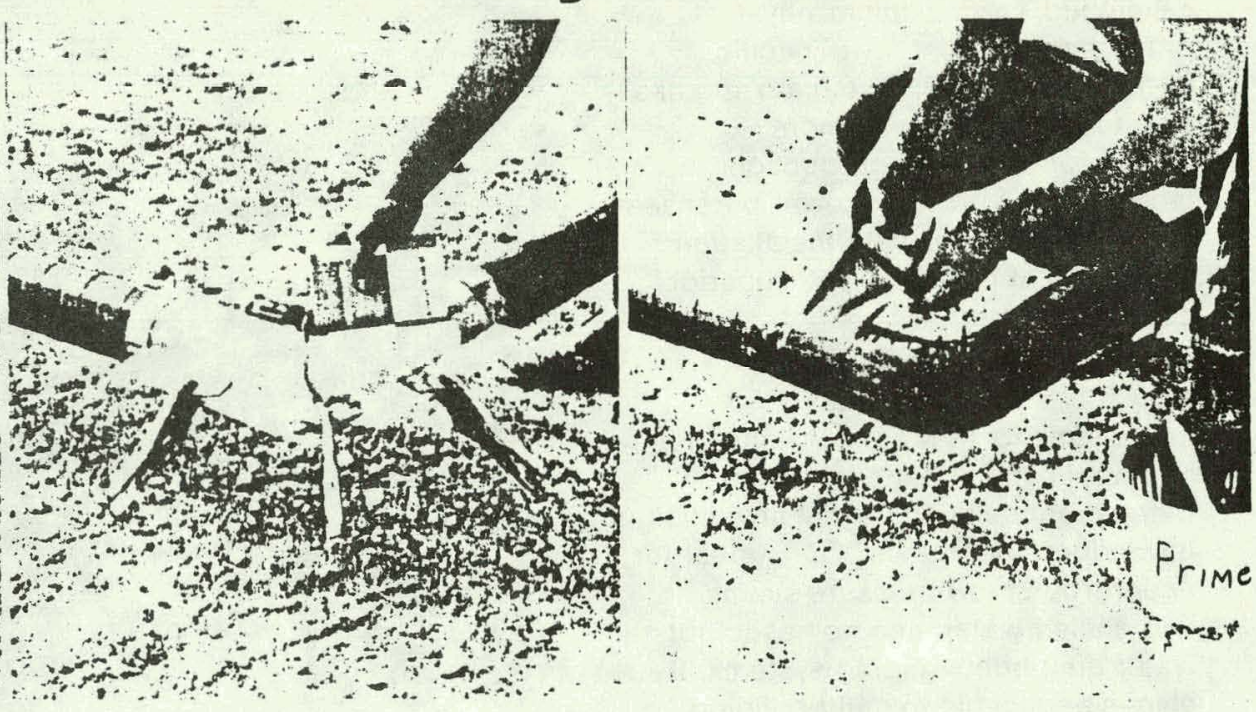

10 Allow approximately 20 minutes for the urethane to foam and cure. Remove the mold, apply primer and polyvinyl chloride-butyl rubber laminate tape securely. Installation is complete. (Tee joints are connected in the same manner.) 


\section{Technical data}

Physical Properties of Thermacor C

Thermal Conductivity

0.13

(k-factor)

Moisture Absorption (\%) $\quad 0.3 \%$

Resistance to heat Internal

Jacket

Tensil Sirength

Compressive strength

Electrical Properties

Dielectric Strength

Internal

Jacket volts $/ \mathrm{mil}$

Density
250

$275 \mathrm{~F}$

180 Deg F (max)

56 psi

30-60 psi depending

on density

103 CP S. 1.06

$650-850$

2.2 Lbs

Schicatiale to Bloct Sterl Pipe I" Fnsulation Thicknoss

Urethane Foam-Thermacor Insulating Material

Moisture vapor Transmission - (grains/hr/sq $\mathrm{ft} /$ in) (cut Surface) 2-3 perm-in

Water Absorption - $.05 \mathrm{lbs} / \mathrm{sq} \mathrm{ft}$ cut surface Moisture pickup of

material having barrier

film, submerged under

$8 \mathrm{ft}$ head for 4 years

Bond to pipe

Negligible

Solvent Resistance

Conc. Alkali

Dil. Alkali

Hydrocarbons

Dil. Acids

Conc. Acids

Degradation by

Fungus

Excellent

Degradation by Molds

Excellent

Excellent

Good to Excellent

Good

Poor

Good

Good

Polyvinyl Chloride-Butyl Rubber Laminate Tape

Shock Impact

Abrasion

Weathering

Acids, non-oxidizing

Alkalis

Alcohol

Hydrocarbons
Excellent

Excellent

Excellent

Excellent

Good

Fair to Excellent

Fair to Excellent
Bacteria

Fungi

Vapor Transmission

Tensil Strength

Tear Strength
Excellent

Excellent

$.002-.05$ grams/100

sq. inches $/ 24 \mathrm{hrs}$.

2800-3500 psi

350-475 Lbs./in.
Thermacor " $C$ " suggested thicknesses for proper insulation

\begin{tabular}{|c|c|c|c|c|c|c|c|c|c|c|c|}
\hline \multirow{2}{*}{$\begin{array}{c}\text { Nom. } \\
\text { 1.P.S. }\end{array}$} & -300 & -250 & -200 & -150 & -100 & -60 & -40 & -20 & 0 & 20 & 40 \\
\hline $1 / 2$ & 2 & 2 & 2 & $11 / 2$ & $11 / 2$ & $11 / 2$ & $11 / 2$ & 1 & & & \\
\hline $1 / 2$ & $21 / 2$ & 2 & 2 & 2 & $11 / 2$ & $11 / 2$ & $11 / 2$ & 1 & & & \\
\hline 1 & $21 / 2$ & $21 / 2$ & 2 & 2 & $11 / 2$ & $11 / 2$ & $11 / 2$ & 1 & 1 & & \\
\hline $11 / 6$ & 3 & $21 / 2$ & 2 & 2 & $11 / 2$ & $11 / 2$ & $11 / 2$ & $11 / 2$ & 1 & & \\
\hline $11 / 2$ & 3 & $21 / 2$ & 2 & 2 & $11 / 2$ & $11 / 2$ & $11 / 2$ & $11 / 2$ & 1 & & \\
\hline 2 & 3 & $21 / 2$ & $21 / 2$ & 2 & $11 / 2$ & $11 / 2$ & $11 / 2$ & $11 / 2$ & 1 & & \\
\hline $21 / 2$ & 3 & $21 / 2$ & $21 / 2$ & 2 & $11 / 2$ & $11 / 2$ & $11 / 2$ & $11 / 2$ & 1 & & \\
\hline 3 & 3 & 3 & $21 / 2$ & $21 / 2$ & 2 & $11 / 2$ & $11 / 2$ & $11 / 2$ & 1 & 1 & \\
\hline $31 / 2$ & 3 & 3 & $21 / 2$ & $21 / 2$ & 2 & $11 / 2$ & $11 / 2$ & $11 / 2$ & 1 & 1 & \\
\hline 4 & $31 / 2$ & 3 & $21 / 2$ & $21 / 2$ & 2 & $11 / 2$ & $11 / 2$ & $11 / 2$ & 1 & 1 & 1 \\
\hline $41 / 2$ & $31 / 2$ & 3 & $21 / 2$ & $21 / 2$ & 2 & $11 / 2$ & $11 / 2$ & $11 / 2$ & 1 & 1 & 1 \\
\hline 5 & $31 / 2$ & 3 & 3 & $21 / 2$ & 2 & 2 & $11 / 2$ & $11 / 2$ & $11 / 2$ & 1 & 1 \\
\hline 6 & $31 / 2$ & $31 / 2$ & 3 & $21 / 2$ & $21 / 2$ & 2 & $11 / 2$ & $11 / 2$ & $11 / 2$ & 1 & 1 \\
\hline 8 & 4 & $31 / 2$ & 3 & $21 / 2$ & $21 / 2$ & 2 & $11 / 2$ & $11 / 2$ & $11 / 2$ & 1 & 1 \\
\hline
\end{tabular}

Cold pipe insulation thicknesses based on $90^{\circ} \mathrm{F}$. ambient air, $80 \%$ relative humidity, zero M.P.H. velocity, and a minimum surface temperature of $84^{\circ} \mathrm{F}$.
Graph showing

Thermal Conductivity of Thermacor " $C$ " Thermacor vs. other insulations.

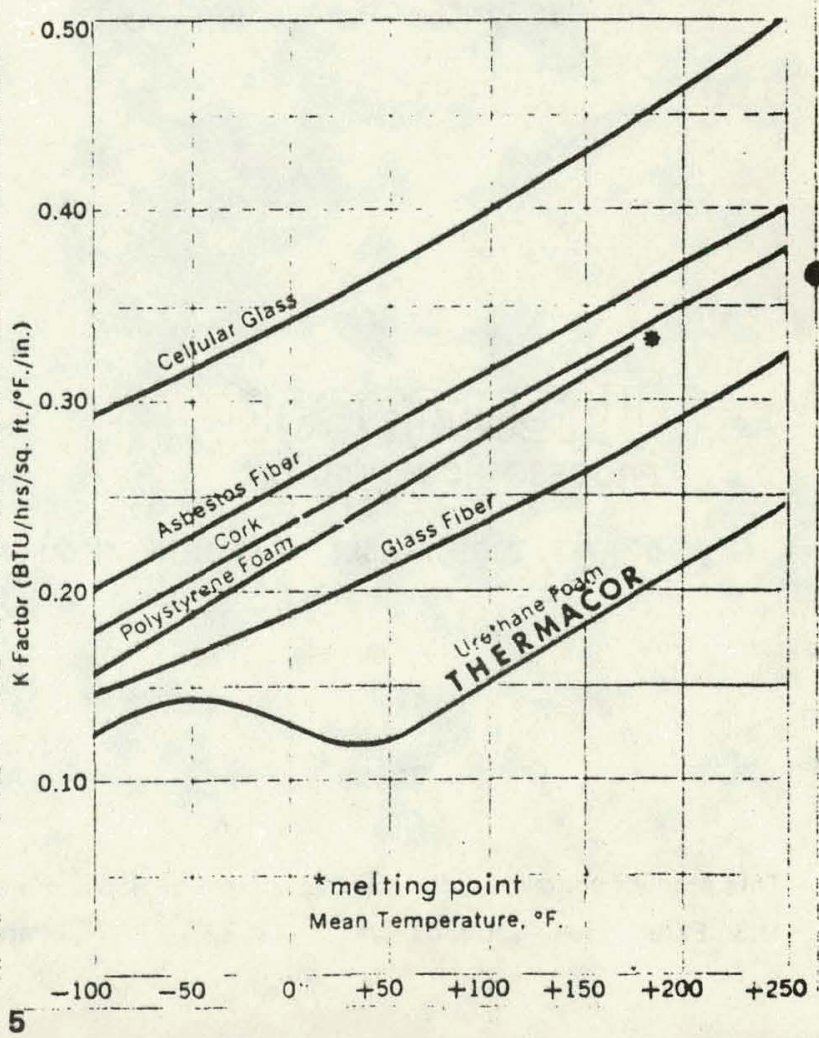




\section{Specifications}

All underground lines shall be factory coated with a $1^{\prime \prime}$ or $2^{\prime \prime} \pm 1 / 8$ " thick closed cell urethane foam with an integral vapor barrier film of high density urethane, and an outer wrapping of primer and 20 mil laminated butyl rubber/polyvinyl tape, as an outer vapor barrier, producing the following physical properties:

1 Minimum density of core 2 ibs/ cubic $\mathrm{ft}$.

2 Minimum compressive strength of core 30 psi.

3 Minimum average compressive strength of coating $48 \mathrm{psi}$.

$1 \mathrm{~K}$ factor 0.13 at $50^{\circ} \mathrm{F}$ mean temperature.

5 Water vapor transmission of barrier film at 0.01 perm-in.

6 Water vapor transmission of core 2 to 3 perm-in.

7 Dielectric strength $103 \mathrm{CP}$ S 1.06 .

8 Fully bonded and continuous throughout the length of the pipe with 6" cut back each end of each joint.

Joints and fittings shall be coated in the field with the same material and to the same thickness as the pipe, using methods and procedures as recommended by THERMACOR PROCESS INC. of Fort Worth, Texas.

\section{Therrnacors. PROCESS INCORPORATED}

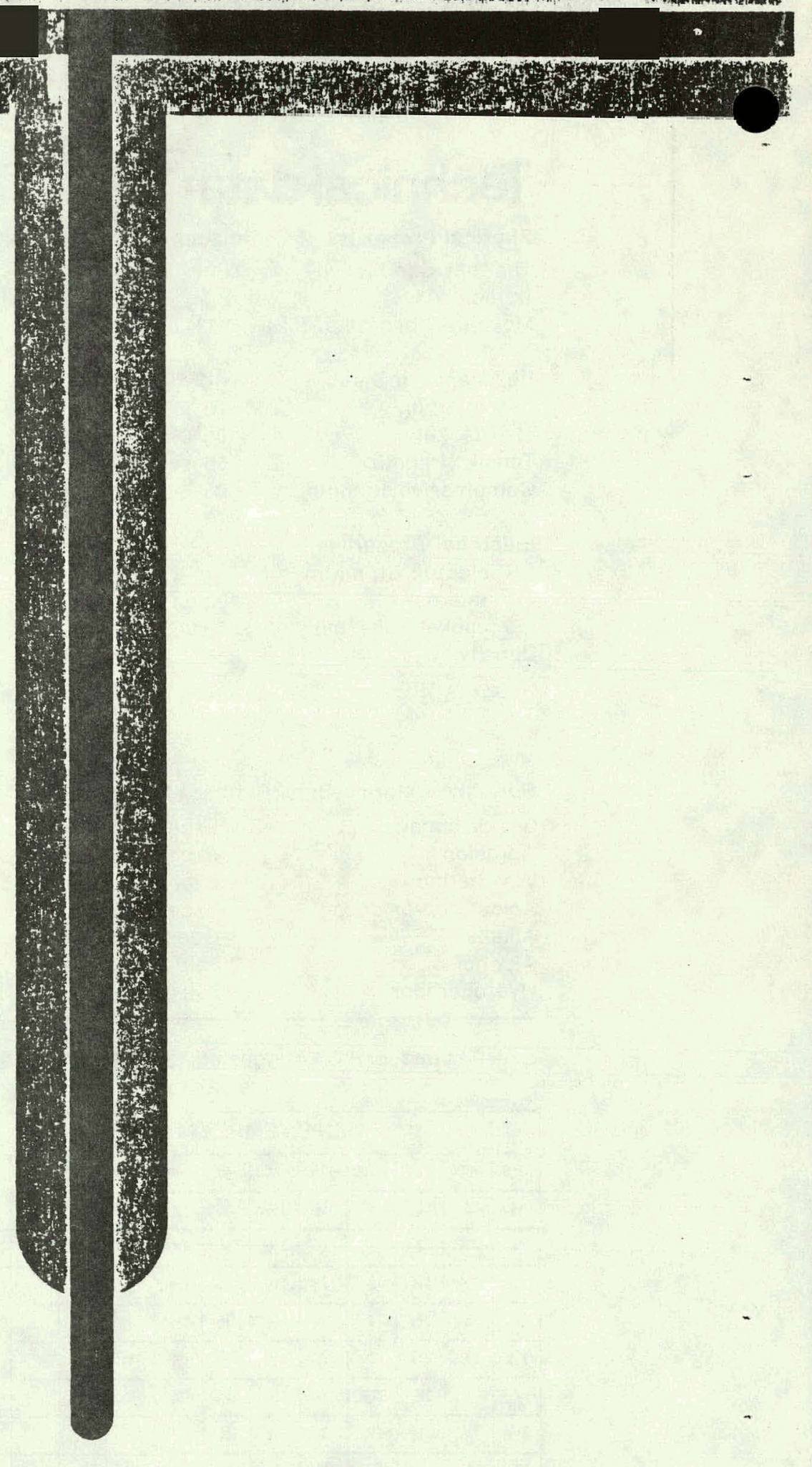

500 N.E. 23RD STREET, FT. WORTH, TEXAS 76106, TELEPHONE A.C. 817 624-1181 


\section{THERMAL PRODUCTS, INC.}

P. . B. BOX 736 - PHONE TE 8.4070

WICHITA, RANSAS 67204

Date February 16

MECHANICAL INSULATION SUBMITTAL

PROJECT: Solar Energy Application LOCATION:K-12 School Building, Mentor, Ks .

REFER TO:

SURFACES TO INSULATE:

Solar water and heat pump system water piping

MATERLAL TO BE USED:

17" thick fiberglass pipe insulation reed oumutac ox: undergraind youing

MANUEACTURED BY: in Dulation.

Certain-Teed Corp.

ADHESIVE OR METHOD OF ATTACHMENT:

Self seal lap

MATERIAL FOR FITTINGS:

Insulating cement to same thickness as adjacent pipe insulation, finished smooth and canvassed with $4 \mathrm{oz}$. canvas. Fittings exposed to weather shall be weatherproofed with Childers CP-10 weatherproof couting, reinforced with glass fabric.

TYPE OF FINISH:

White All Service Jacket (ASJ) on all piping. Pliping exposed to weather shall have .016 aluminum jacket.

Not required

PAINTING:

NOTES OR REMARKS:

Convertor and alr separator shall be covered with $1 \frac{1}{2}$ " thick flberglass same as plping.
[1] ArPringen

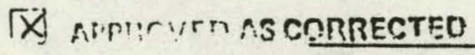
mi: A PID RESUEMIT . . . T! …
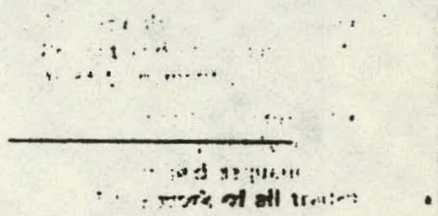

amar 78 a imn III ymh. 


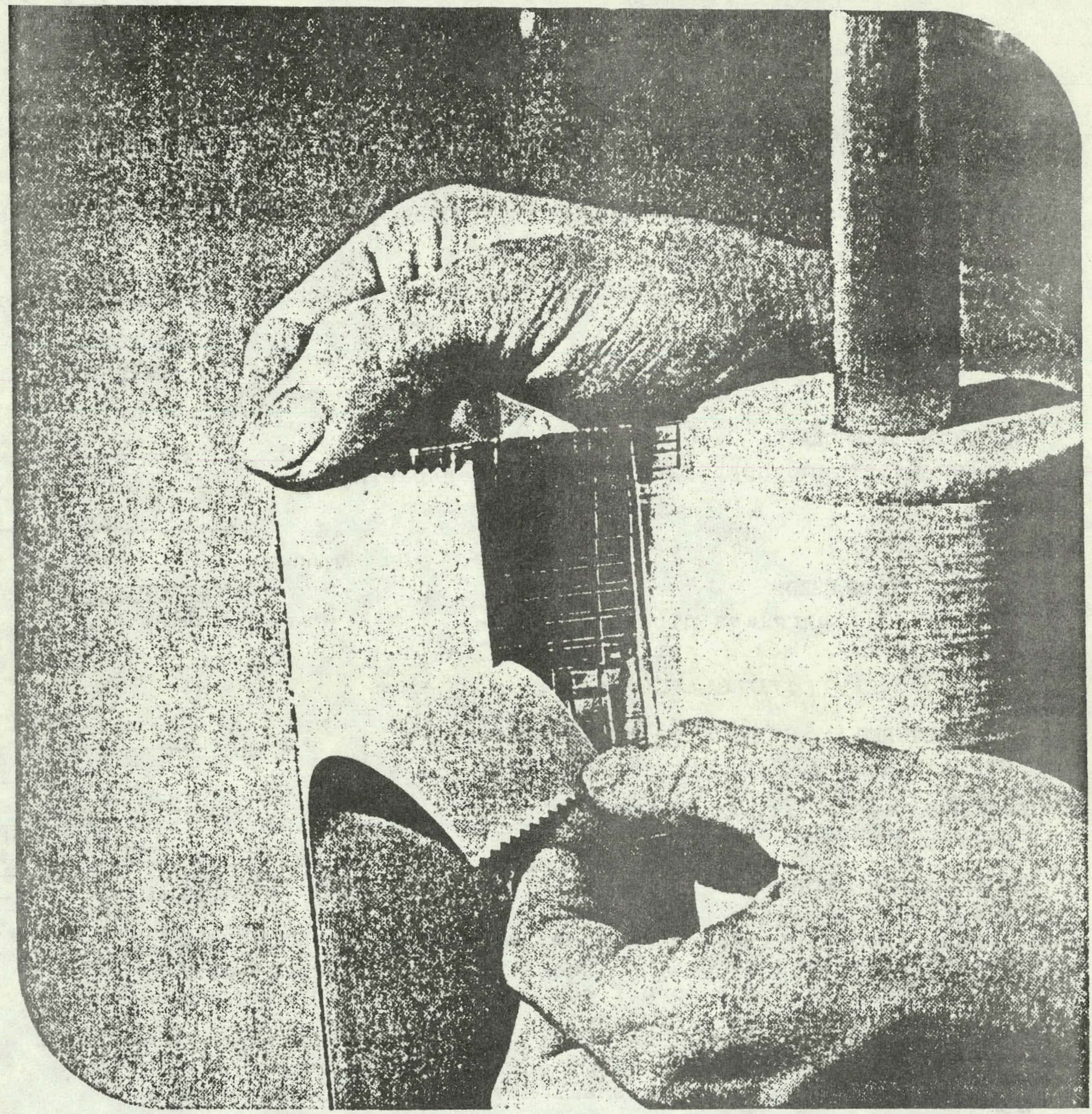

ASJ JACKET SHOWN WITH FACTOPY APPLIED SSL TAPE, ALSO AVAILABLE WITHOUT SSL TAPE

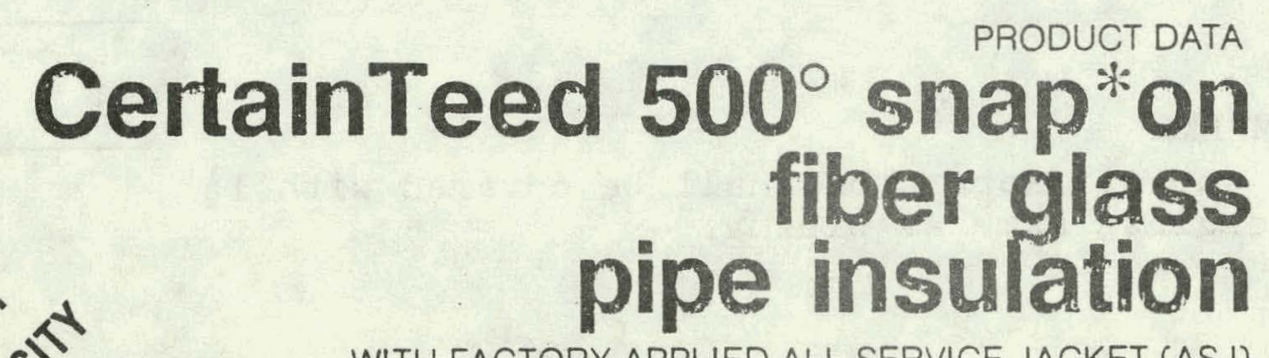

WITH FACTORY APPLIED ALL SERVICE JACKET (ASJ)

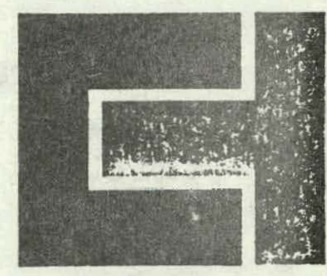

CEETANTIEED 


\section{Description}

CertainTeed $500^{\circ}$ Snap *On Pipe Insulation is composed of extremely fine diameter glass fibers bonded together with a phenolic resin and molded in one piece sections. Made with a single seam. CertainTeed Snap* On spreads open to receive the pipe and snaps quickly in place. it has all the desirable features of glass. The glass fiber will not burn, is not affected by moisture, will not corrode metals. will not rot or mildew and is permanent. The insulation will permit expansion and contraction of the pipe without cracking and it will not shrink CertainTeed $500^{\circ}$ Snap* On Pipe Insulation with factory applied ALL SERVICE JACKET is specifically designed for dual temperature piping. The product may be used on lines operating from $-20 \mathrm{~F}$ to $500 \mathrm{~F}$.

\section{Uses}

Dumestic Hot Water Heating Hot Water High Temperature Hot Water

Chilled Water

Dual Temperature

\section{Thermal Efficiency}

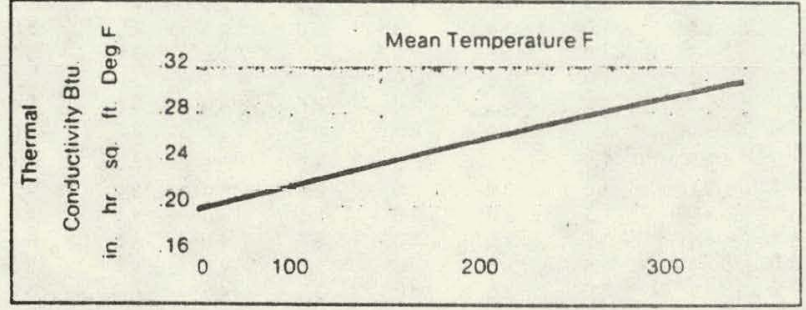

\section{Benefits}

- Quick and clean application

- Flame resistant

- High thermal efficiency

- Vapor barrier not broken as need for staples is eliminated

- Meets the fire and safety requirements of building codes and government guide specifications

\section{Specification Compliances}

CertainTeed $500^{\circ}$ Snap* On Pipe Insulation with factory applied ALL SERVICE JACKET meets the following specifications:

HH-B-100B. Types I and II. (Jacket)

HH-l-558B, Form D, Type III, Class 12 (10 450F)

Class 13 (to 500F)

\section{MIL-1-22344B}

ASTM C547-67

\section{U.L. Listing}

CertainTeed $500^{\circ}$ Snap*On with factory applied ALL SERVICE JACKET is listed by Underwriters' Laboratories as having a composite (pipe covering and jacket) flame hazard classification not to exceed the following

Flame Spread 25 Fuel Contributed 50

Smoke Developed 50

\section{Jacket Properties}

- Laminated aluminum foil, glass reinforcing and white kraft paper

- Perm rating of 0.01 perms (HH-B-100B. Type I)

- Puncture resistance: minimum 50 (Beach puncture)

- Tensile strength: $35 \mathrm{lbs}$ //in.

\section{$850^{\circ}$ Snap* On}

CertainTeed also manufactures a Snap*On Pipe Insulation for service temperatures up to $850^{\circ} \mathrm{F}$. Description of this extremely efficient fiber glass insulation for these temperatures is covered in data sheet \#30-31-49U
Recommendations For Installing CertainTeed $500^{\circ}$ Snap* On With Self Sealing ASJ Jacket

1. Make certain ASJ Jacket is clean.

2. Make all cuts and fabrications before removing release paper.

3. Make sure to rub hard to assure a positive seal.

4. Do not install when air temperature is Inwer than $3.5 \mathrm{~F}$ nr nver 1 ? $\mathrm{F}$.

5. Do not leave adhesive strip exposed to the air. Adhere self sealing lap immediately after removing paper backing.

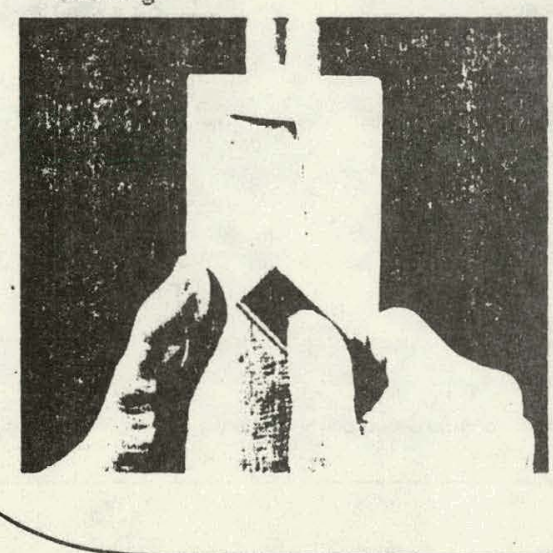

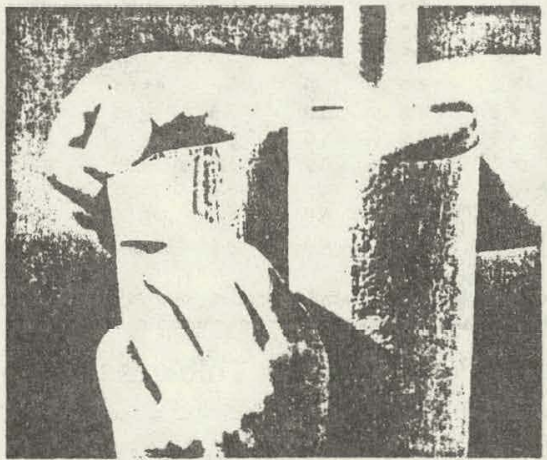
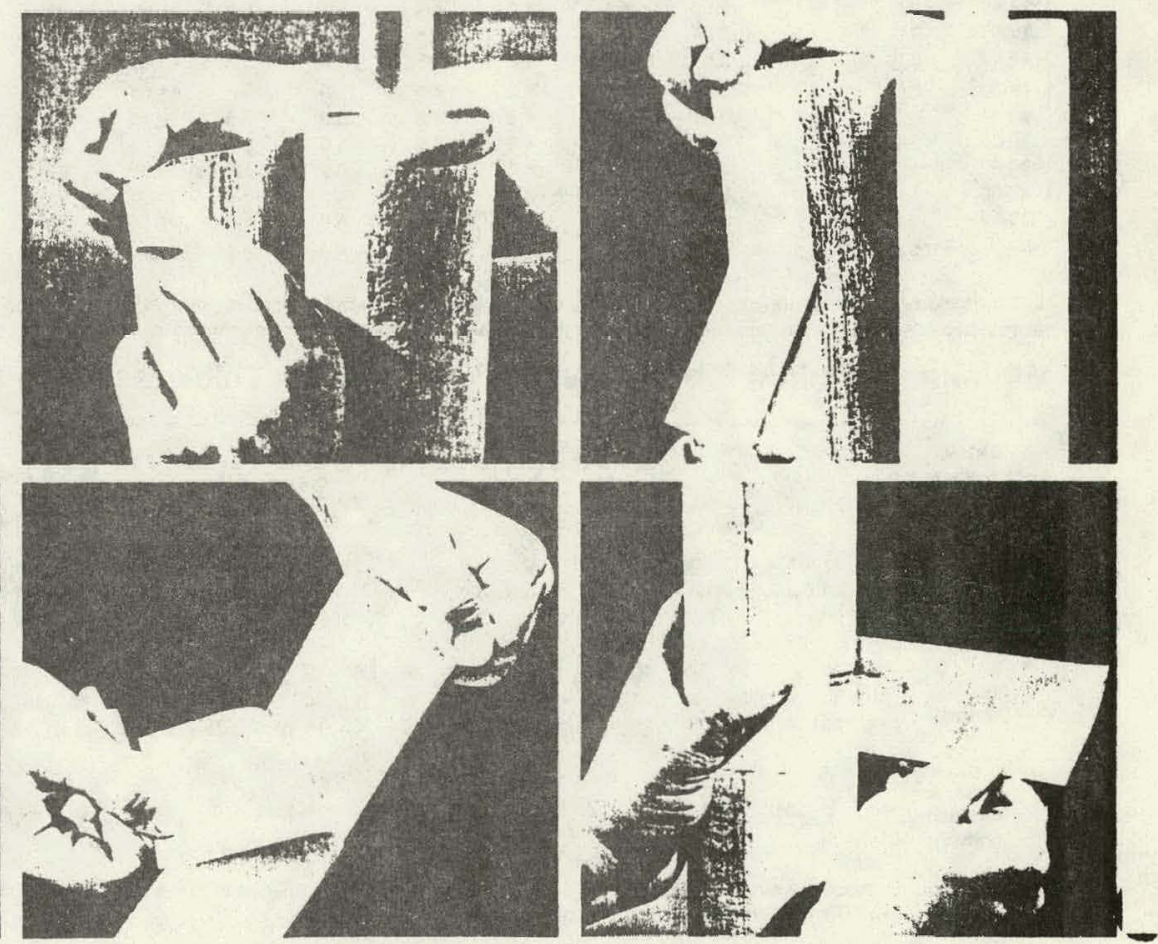
Recommended Thicknesses* Heated Piping Insulation

\begin{tabular}{|c|c|c|c|}
\hline \multicolumn{4}{|c|}{ Commerclal (fiull time) } \\
\hline Size & 150 & 200 & 250 \\
\hline 8.50 & 0.5 & 1.0 & 1.5 \\
\hline 0.75 & 1.0 & 1.0 & 1.5 \\
\hline 1.00 & 1.0 & 1.5 & 1.5 \\
\hline 1.25 & 1.0 & 1.5 & 2.0 \\
\hline 1.50 & 1.0 & 1.5 & 2.0 \\
\hline 2.00 & 1.5 & 2.0 & 2.5 \\
\hline 2.50 & 1.5 & 2.0 & 2.5 \\
\hline 3.60 & 1.5 & 2.0 & 2.5 \\
\hline 9.50 & 1.5 & 2.5 & 3.0 \\
\hline $\begin{array}{l}4.00 \\
4.50\end{array}$ & 1.5 & 2.5 & 3.0 \\
\hline 8.00 & 2.0 & 2.5 & 3.0 \\
\hline 6.60 & 2.0 & 2.5 & 3.5 \\
\hline 7.00 & 2.0 & 3.0 & 3.5 \\
\hline 8.00 & 2.0 & 3.0 & 3.5 \\
\hline 9.00 & 2.0 & 3.0 & 3.5 \\
\hline 10.00 & 2.0 & 3.0 & 4.0 \\
\hline 12.00 & 2.5 & 3.0 & 1.0 \\
\hline 14.00 & 2.0 & 3.0 & 4.0 \\
\hline 16.00 & 2.0 & 3.0 & 4.0 \\
\hline 18.00 & 20 & 3.0 & 4.0 \\
\hline 20.00 & 2.0 & 3.0 & 3.5 \\
\hline 24.00 & 2.0 & 3.0 & 3.5 \\
\hline 30.00 & 2.0 & 2.5 & 3.5 \\
\hline 36.00 & 1.5 & 2.5 & 3.0 \\
\hline Flat & 2.5 & 3.5 & 4.5 \\
\hline
\end{tabular}

Operating Temperature (F)

Process (full time) Operating Temperature (F)

Commercial (pant tume) Operating Temperature (F)

$\begin{array}{rrrrr}2.5 & 3.0 & 0.50 & 0.5 & 1.0 \\ 3.0 & 3.0 & 0.75 & 0.5 & 1.0 \\ 3.0 & 3.5 & 1.00 & 0.5 & 1.0 \\ 3.5 & 4.0 & 1.25 & 1.0 & 1.0 \\ 3.5 & 4.0 & 1.50 & 1.0 & 1.5 \\ 4.0 & 4.5 & 2.00 & 1.0 & 1.5 \\ 4.5 & 5.0 & 2.50 & 1.0 & 1.5 \\ 4.5 & 5.0 & 3.00 & 1.0 & 1.5 \\ 5.0 & 5.5 & 3.50 & 1.5 & 2.0 \\ 5.0 & 5.5 & 4.00 & 1.5 & 2.0 \\ 5.0 & 6.0 & 8.50 & 1.5 & 2.0 \\ 5.5 & 6.0 & 5.00 & 1.5 & 2.0 \\ 5.5 & 6.5 & 6.00 & 1.5 & 2.0 \\ 6.0 & 6.5 & 7.00 & 1.5 & 2.0 \\ 6.0 & 6.5 & .00 & 1.5 & 2.5 \\ 6.0 & 7.0 & 8.00 & 1.5 & 2.5 \\ 6.5 & 7.0 & 10.00 & 1.5 & 2.5 \\ 6.5 & 7.0 & 12.00 & 2.0 & 2.5 \\ 6.5 & 70 & 14.00 & 2.0 & 2.5 \\ 6.5 & 7.0 & 16.00 & 2.0 & 2.5 \\ 6.5 & 7.0 & 1.00 & 2.0 & 2.5 \\ 6.0 & 7.0 & 20.00 & 1.5 & 2.5 \\ 6.0 & 6.5 & 24.00 & 1.5 & 2.5 \\ 5.5 & 6.0 & 30.00 & 1.5 & 2.0 \\ 5.0 & 5.5 & 36.00 & 1.5 & 2.0 \\ 7.5 & 8.0 & 710 & 2.0 & 3.0\end{array}$

$\begin{array}{llllll}250 & 300 & 350 & 400 & 450 & 500 \\ 1.0 & 1.5 & 1.5 & 1.5 & 2.0 & 2.0 \\ 1.0 & 1.5 & 1.5 & 2.0 & 2.0 & 2.5 \\ 1.5 & 1.5 & 2.0 & 2.0 & 2.5 & 2.5 \\ 1.5 & 2.0 & 2.0 & 2.5 & 2.5 & 30 \\ 1.5 & 2.0 & 2.0 & 2.5 & 3.0 & 3.0 \\ 2.0 & 2.0 & 2.5 & 3.0 & 3.0 & 3.5 \\ 2.0 & 2.5 & 2.5 & 3.0 & 3.5 & 3.5 \\ 20 & 2.5 & 3.0 & 3.5 & 3.5 & 4.0 \\ 2.0 & 2.5 & 3.0 & 3.5 & 4.0 & 4.0 \\ 2.5 & 2.5 & 3.0 & 3.5 & 4.0 & 4.5 \\ 2.5 & 3.0 & 3.5 & 3.5 & 4.0 & 4.5 \\ 2.5 & 3.0 & 3.5 & 4.0 & 4.5 & 4.5 \\ 2.5 & 3.0 & 3.5 & 4.0 & 4.5 & 5.0 \\ 3.0 & 3.5 & 3.5 & 4.0 & 4.5 & 5.0 \\ 3.0 & 3.5 & 4.0 & 4.5 & 5.0 & 5.5 \\ 3.0 & 3.5 & 4.0 & 4.5 & 5.0 & 5.5 \\ 3.0 & 3.5 & 4.0 & 4.5 & 5.0 & 5.5 \\ 0.0 & 3.5 & 4.0 & 4.5 & 5.0 & 5.5 \\ 3.0 & 3.5 & 4.0 & 4.5 & 5.0 & 5.5 \\ 3.0 & 3.5 & 4.0 & 4.5 & 5.0 & 5.5 \\ 3.0 & 3.5 & 4.0 & 4.5 & 5.0 & 5.5 \\ 3.0 & 3.5 & 4.0 & 4.5 & 5.0 & 5.5 \\ 3.0 & 3.5 & 4.0 & 4.5 & 5.0 & 5.0 \\ 2.5 & 3.0 & 3.5 & 4.0 & 4.5 & 5.0 \\ 2.5 & 3.0 & 3.0 & 3.5 & 4.0 & 4.5 \\ 3.5 & 4.0 & 5.0 & 5.5 & 6.0 & 6.5\end{array}$

\begin{tabular}{|c|c|c|c|}
\hline stza & 150 & 200 & 250 \\
\hline 0.50 & 0.5 & 1.0 & 1.0 \\
\hline $\begin{array}{l}0.75 \\
1.00\end{array}$ & $\begin{array}{l}0.5 \\
0.5\end{array}$ & $\begin{array}{l}1.0 \\
1.0\end{array}$ & $\begin{array}{l}1.0 \\
1.5\end{array}$ \\
\hline 1.25 & 1.0 & 1.0 & \\
\hline 1.50 & 1.0 & 1.5 & $\begin{array}{l}1.5 \\
20\end{array}$ \\
\hline $\begin{array}{l}2.00 \\
2.50\end{array}$ & $\begin{array}{l}1.0 \\
1.0\end{array}$ & $\begin{array}{l}1.5 \\
1.5\end{array}$ & $\begin{array}{r}2.0 \\
2.0\end{array}$ \\
\hline 3.00 & 1.0 & 1.5 & 2.0 \\
\hline 3.50 & 1.5 & 2.0 & 2.0 \\
\hline 4.00 & 1.5 & 2.0 & 2.5 \\
\hline 4.30 & 1.5 & 2.0 & 2.5 \\
\hline 5.00 & 1.5 & 2.0 & 2.5 \\
\hline 6.00 & 1.5 & 2.0 & 2.5 \\
\hline 7.00 & 1.5 & 2.5 & 3.0 \\
\hline 8.00 & 1.5 & 2.5 & 3.0 \\
\hline 9.00 & 1.5. & 2.5 & 3.0 \\
\hline 10.00 & 2.0 & 2.5 & 3.0 \\
\hline 12.00 & 2.0 & 2.5 & 3.0 \\
\hline 14.00 & 2.0 & 2.5 & 3.0 \\
\hline 16.00 & 2.0 & 2.5 & 3.0 \\
\hline 18.00 & 2.0 & 2.5 & 3.0 \\
\hline 20.00 & 2.0 & 2.5 & 3.0 \\
\hline 24.03 & 1.5 & 2.5 & 3.0 \\
\hline 30.00 & 1.5 & 2.0 & 2.5 \\
\hline$\$ 8.00$ & 1.5 & 2.0 & 2.5 \\
\hline Fiat & 2.0 & 3.0 & 3.5 \\
\hline
\end{tabular}

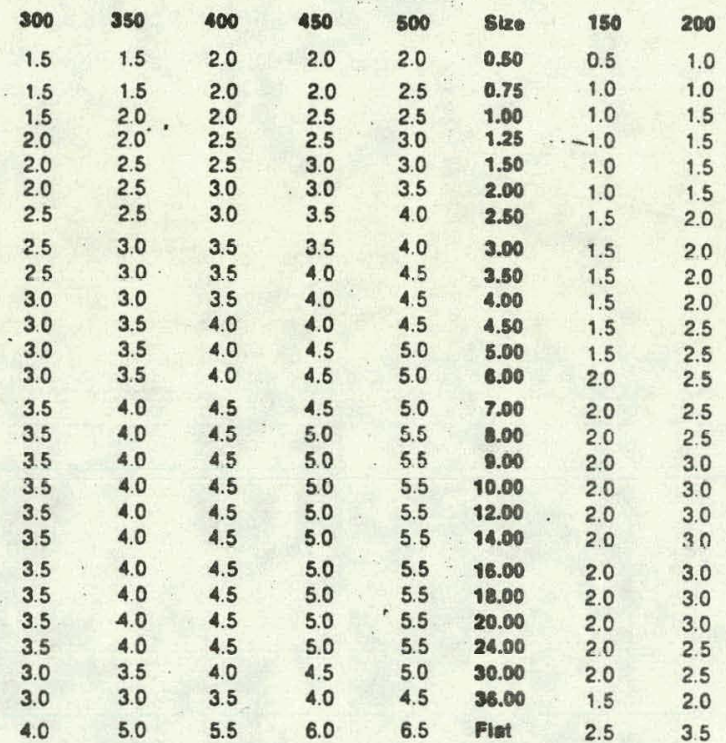

Operating Temperature (F)

- ECON thicknesses appearing above are economic thicknesses under average operating condition. Special conditions may warrant the use of other thicknesses. ECON is the lowest possible sum of the annual cost of heat and the annual cost of insulation as determined by the Thermal Insulation Manufacturers Association (TIMA).

Minimum Nominal Fiber Glass Pipe Insulation Thickness Needed to Prevent Condensation

Tamble Condtaton:

.

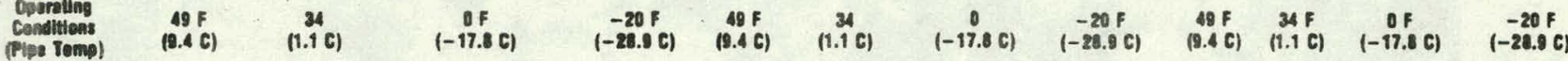

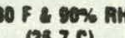

(23.7 C)

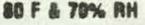

$20 \mathrm{~F}$ : $50 \%$ AH

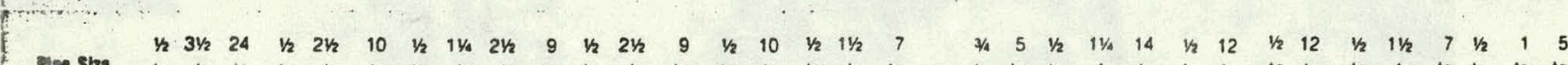

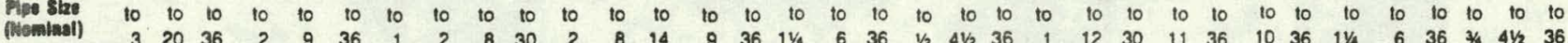

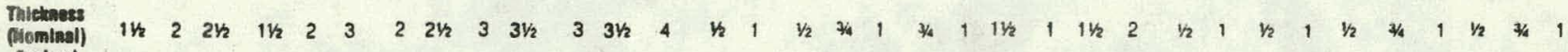
(Rnehes)

This chart was developed to aid designers and buyers in choosing the proper insulation thickness for piping systems. For more details on specific or unusual problems. consult your nearest CertainTeed District Office. Temperature Conversion $-{ }^{\circ} \mathrm{C}=5 / 9\left({ }^{\circ} \mathrm{F}-32\right)$ 


\begin{tabular}{|c|c|c|c|c|c|c|c|c|c|c|c|c|c|c|c|c|c|}
\hline $\begin{array}{l}\text { Nom. } \\
\text { Plpe } \\
\text { Sizes }\end{array}$ & $\begin{array}{l}\text { Ptpo } \\
\text { O.0. }\end{array}$ & $\begin{array}{l}h^{\prime} \text { w } \\
\text { Walt }\end{array}$ & $\begin{array}{l}\text { I Thlek } \\
\text { Inaul. } \\
\text { O.D. }\end{array}$ & $\begin{array}{l}\text { T"Wo } \\
\text { Woll }\end{array}$ & $\begin{array}{c}\text { nThlek } \\
\text { Insul. } \\
\text { O.D. }\end{array}$ & $\begin{array}{l}\text { Is" w } \\
\text { Wall }\end{array}$ & $\begin{array}{c}\text { all Thick } \\
\text { Inaul. } \\
\text { O.D. }\end{array}$ & & $\begin{array}{c}\text { II Thlek } \\
\text { Ineul. } \\
\text { O.d. }\end{array}$ & $\begin{array}{l}2 v_{2}^{\prime} \cdot w \\
\text { WoH }\end{array}$ & $\begin{array}{c}\text { II Thick } \\
\text { Insul. } \\
\text { O.D. }\end{array}$ & $\begin{array}{l}\text { 3' We } \\
\text { Wall }\end{array}$ & $\begin{array}{c}\text { I Thlck } \\
\text { Insul. } \\
\text { O.D. }\end{array}$ & $\begin{array}{c}2 k " n \\
\text { Wall }\end{array}$ & $\begin{array}{c}\text { sit Thick } \\
\text { Insul. } \\
\text { O.D. }\end{array}$ & $\begin{array}{l}3 \cdot \text { wo } \\
\text { Wall }\end{array}$ & $\begin{array}{l}\text { II Thick } \\
\text { Insul. } \\
\text { O.D. }\end{array}$ \\
\hline 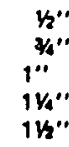 & $\begin{array}{r}.84 \\
1.05 \\
1.32 \\
1.66 \\
1.90\end{array}$ & $\begin{array}{l}.51 \\
.63 \\
.49 \\
.60 \\
.48\end{array}$ & $\begin{array}{l}1.90 \\
2.38 \\
2.38 \\
2.88 \\
2.88\end{array}$ & $\begin{array}{r}.99 \\
.88 \\
1.06 \\
.91 \\
1.04\end{array}$ & $\begin{array}{l}2.88 \\
2.88 \\
3.50 \\
3.50 \\
4.00\end{array}$ & \begin{tabular}{|l}
1.55 \\
1.44 \\
1.56 \\
1.66 \\
1.54
\end{tabular} & $\begin{array}{l}4.00 \\
4.00 \\
4.50 \\
5.00 \\
5.00\end{array}$ & $\begin{array}{l}2.05 \\
1.94 \\
2.09 \\
1.93 \\
1.82\end{array}$ & $\begin{array}{l}5.00 \\
5.00 \\
5.56 \\
5.56 \\
5.56\end{array}$ & $\begin{array}{l}\bar{z} \\
\bar{z}\end{array}$ & $\begin{array}{l}z \\
z \\
-\end{array}$ & $\begin{array}{l}\bar{z} \\
\bar{z}\end{array}$ & $\begin{array}{l}\bar{z} \\
\bar{z} \\
\bar{z}\end{array}$ & $\begin{array}{l}2.35 \\
2.78 \\
2.65 \\
2.48 \\
2.36 \\
\end{array}$ & \begin{tabular}{|l|}
5.56 \\
6.63 \\
6.63 \\
6.63 \\
6.63 \\
\end{tabular} & $\begin{array}{l}2.87 \\
3.01 \\
3.13 \\
2.95 \\
2.83\end{array}$ & $\begin{array}{l}6.63 \\
7.63 \\
7.63 \\
7.63 \\
7.63 \\
\end{array}$ \\
\hline & $\begin{array}{l}2.38 \\
2.88\end{array}$ & $\begin{array}{l}.55 \\
.55\end{array}$ & $\begin{array}{l}3.50 \\
4.00\end{array}$ & $\begin{array}{l}1.05 \\
1.05\end{array}$ & $\begin{array}{l}4.50 \\
5.00\end{array}$ & $\begin{array}{l}1.58 \\
1.33\end{array}$ & $\begin{array}{l}5.56 \\
5.56\end{array}$ & \begin{tabular}{|l}
2.11 \\
1.86 \\
\end{tabular} & $\frac{6.63}{6.63}$ & $\begin{array}{l}2.62 \\
2.36\end{array}$ & $\begin{array}{l}7.63 \\
7.63\end{array}$ & $\begin{array}{l}3.11 \\
2.86\end{array}$ & $\begin{array}{l}8.63 \\
8.63\end{array}$ & & & & \\
\hline $\begin{array}{l}3^{\prime \prime \prime} \\
3 V^{\prime \prime \prime}\end{array}$ & $\begin{array}{l}3.50 \\
4.00\end{array}$ & $\begin{array}{l}.70 \\
.70\end{array}$ & $\begin{array}{l}5.00 \\
5.56 \\
5.56\end{array}$ & $\begin{array}{r}.95 \\
1.23 \\
\end{array}$ & $\begin{array}{r}5.56 \\
6.63 \\
\end{array}$ & $\frac{1.48}{1.23}$ & $\frac{6.63}{6.63}$ & $\begin{array}{l}1.98 \\
1.73 \\
\end{array}$ & $\begin{array}{l}7.63 \\
7.63 \\
8.63\end{array}$ & $\begin{array}{l}2.48 \\
2.73 \\
2.54\end{array}$ & $\begin{array}{l}8.63 \\
9.63 \\
9.63\end{array}$ & $\begin{array}{l}2.98 \\
2.73 \\
\end{array}$ & $\begin{array}{r}9.63 \\
9.63 \\
.67\end{array}$ & \multirow{3}{*}{\multicolumn{4}{|c|}{$\begin{array}{l}\text { Standard Lengths are 3-foot } \\
\text { sections. All sizes below } \\
\text { solid ( ) line available } \\
\text { on request in uniacketed } \\
6 \text {-foot sections. At factory } \\
\text { option } 10 \% \text { of 6-toot } \\
\text { sections may be lurnished } \\
\text { in 3-toot sections. }\end{array}$}} \\
\hline 4" & 4.50 & .51 & 5.56 & 1.04 & 6.63 & 1.54 & 7.63 & 2.04 & 8.63 & 2.54 . & 9.63 & 3. 10 & 10.75 & & & & \\
\hline $\begin{array}{c}44^{\prime \prime} \\
5^{\prime \prime} \\
6^{\prime \prime} \\
77^{\prime \prime} \\
8^{\prime \prime} \\
9^{\prime \prime} \\
10^{\prime \prime} \\
11^{\prime \prime \prime} \\
12^{\prime \prime} \\
14^{\prime \prime \prime} \\
15^{\prime \prime} \\
16^{\prime \prime}\end{array}$ & \begin{tabular}{|r}
5.00 \\
5.56 \\
6.63 \\
7.63 \\
8.63 \\
9.63 \\
10.75 \\
11.75 \\
12.75 \\
14.00 \\
15.00 \\
16.00 \\
\end{tabular} & $\begin{array}{l}.79 \\
.75 \\
.50 \\
= \\
= \\
= \\
= \\
= \\
=\end{array}$ & $\begin{array}{l}6.63 \\
7.12 \\
7.63 \\
= \\
= \\
= \\
\overline{ } \\
\overline{ } \\
\overline{-}\end{array}$ & $\begin{array}{r}1.29 \\
1.00 \\
1.00 \\
.96 \\
1.02 \\
1.02 \\
.95 \\
1.07 \\
1.06 \\
.93 \\
.93 \\
.93\end{array}$ & $\begin{array}{r}7.63 \\
7.63 \\
8.63 \\
9.63 \\
10.75 \\
11.75 \\
12.75 \\
14.00 \\
15.00 \\
16.00 \\
17.00 \\
18.00 \\
\end{array}$ & $\begin{array}{l}1.29 \\
1.50 \\
1.50 \\
1.53 \\
1.52 \\
1.52 \\
1.57 \\
1.57 \\
1.56 \\
1.43 \\
1.43 \\
1.43\end{array}$ & \begin{tabular}{|r}
7.63 \\
8.63 \\
9.63 \\
10.75 \\
11.75 \\
12.75 \\
14.00 \\
15.00 \\
1600 \\
17.00 \\
18.00 \\
19.00 \\
\end{tabular} & $\begin{array}{l}1.79 \\
2.00 \\
2.07 \\
2.03 \\
2.02 \\
2.14 \\
2.07 \\
2.07 \\
2.06 \\
1.93 \\
1.93 \\
1.93\end{array}$ & $\begin{array}{r}8.63 \\
9.63 \\
10.75 \\
11.75 \\
12.75 \\
14.00 \\
15.00 \\
16.00 \\
17.00 \\
18.00 \\
19.00 \\
20.00\end{array}$ & $\begin{array}{l}2.85 \\
2.57 \\
2.57 \\
2.53 \\
2.65 \\
2.64 \\
2.57 \\
2.57 \\
2.56 \\
2.43 \\
2.43 \\
2.43\end{array}$ & $\begin{array}{l}10.75 \\
10.75 \\
11.75 \\
12.75 \\
14.00 \\
15.00 \\
16.00 \\
17.00 \\
18.00 \\
19.00 \\
20.00 \\
21.00\end{array}$ & $\begin{array}{l}2.85 \\
3.07 \\
3.07 \\
3.15 \\
3.15 \\
3.14 \\
3.07 \\
3.07 \\
3.06 \\
2.93 \\
2.93 \\
2.93\end{array}$ & \begin{tabular}{|l}
10.75 \\
11.75 \\
12.75 \\
14.00 \\
15.00 \\
16.00 \\
17.00 \\
18.00 \\
19.00 \\
20.00 \\
21.00 \\
22.00
\end{tabular} & & & & \\
\hline $17^{\prime \prime}$ & 00 & - & - & .93 & 1 & 1 & 10 & 1. & 10 & 2.43 & 22.00 & 2.93 & 23.00 & & & & \\
\hline $18^{\prime \prime}$ & & - & - & .93 & & 1. & & & & 2.43 & 23.00 & & & & & & \\
\hline $19^{\prime \prime}$ & .00 & - & - & .93 & 21.00 & 1.43 & 22.00 & 1.93 & 23.00 & 2.43 & 24.00 & 2.93 & 25.00 & & & & \\
\hline $20^{\prime \prime}$ & 0 & - & - & .93 & 22.00 & 1.43 & 23.00 & 1.93 & 24.00 & 2.43 & 25.00 & 2.93 & 26.00 & & & & \\
\hline $21^{\prime \prime}$ & 100 & - & - & .93 & 23.00 & 1.43 & & 1. & & 2.4 & 26.00 & & & & & & \\
\hline $22 "$ & 2.00 & - & - & .93 & 24.00 & 1.43 & 25.00 & 1.9 & 26.00 & 2.43 & 27.00 & 2.93 & 28.00 & & & & \\
\hline $23^{\prime \prime}$ & 3.00 & - & - & .93 & 25.00 & 1. & 2600 & 1.9 & 27 & 2.4 & 28.00 & & & & & & \\
\hline $24^{\prime \prime}$ & 4.00 & - & - & .93 & 26.00 & 1.43 & 27.00 & 1.93 & 28.00 & 2.43 & 29.00 & 2.93 & 30.00 & & & & \\
\hline $25^{\prime \prime}$ & 25.00 & - & - & .93 & 27.00 & 1.43 & 28.00 & 1.93 & 29.00 & 2.43 & 30.00 & 2.93 & 31.00 & & & & \\
\hline $26^{\prime \prime}$ & .00 & - & - & .83 & 28.00 & 1.43 & 29.00 & 1.93 & 30.00 & 2.43 & 31.00 & 2.93 & 32.00 & & ay vary & & \\
\hline $27 "$ & .00 & - & - & .93 & 29.00 & 1.43 & & 1.9 & 31 & 2.43 & 32.00 & 2.93 & 33.00 & & & & \\
\hline $28^{\prime}$ & & - & - & .93 & 30.00 & 1.43 & 31.00 & 1.8 & 32.00 & 2.43 & 33.00 & 2.93 & 34.00 & & & & \\
\hline $29^{\prime \prime}$ & .00 & - & - & .93 & 31.00 & 1.43 & 32.00 & 1. & 33.00 & 2. & 34.00 & 2.93 & 35.00 & & & & \\
\hline $30^{\prime \prime}$ & & - & - & .93 & 32.00 & 1.43 & 33.00 & 1.93 & & 2.43 & 35.00 & 2.93 & & & & & \\
\hline $31^{\prime \prime}$ & 00 & - & - & .93 & 33.00 & 1.43 & 34.00 & 1.93 & 35.00 & 2.43 & 36.00 & 2.93 & 37.00 & & & & \\
\hline 32"' & & - & - & .93 & 34.00 & 1.43 & 35.00 & 1.93 & 36. & 2.43 & 37.00 & 2.93 & & & & & \\
\hline $33^{\prime \prime}$ & & - & - & .93 & 35.00 & 1.43 & 36.00 & 1.93 & 37.00 & 2.43 & 38.00 & 2.93 & 39.00 & & & & \\
\hline & & - & 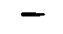 & .93 & 38.00 & 1.43 & 39.00 & - & - & - & - & - & - & & & & \\
\hline
\end{tabular}

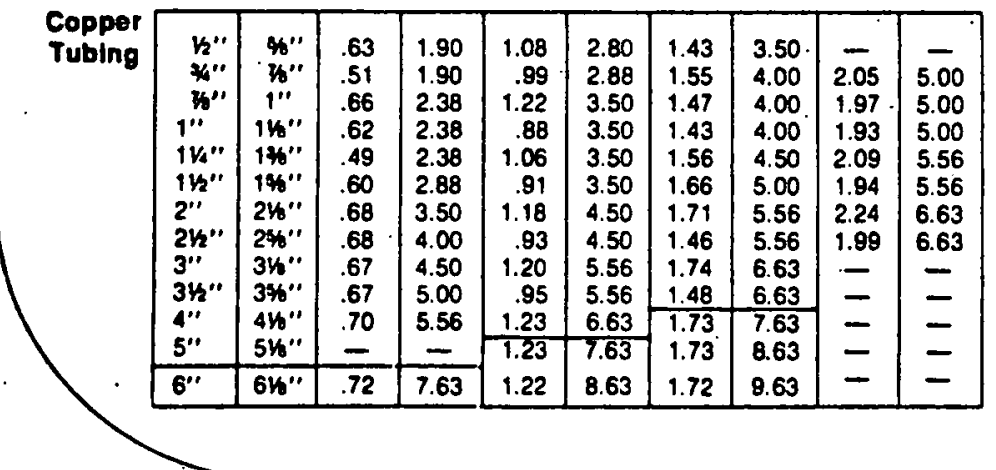

Standard Lengths are 3 -foot sections. All sizes below solid (___ ) line available on request in unjacketed 6-10ot sections. Al factory option 10\% of 6 -foot sections may be furnished in 3-loot sections.

\section{SALES DISTRICTS}

\section{ATLANTA DISTAICT}

P. O. Box 80725

5587 New Peachtree Road Atlanta, GA 30366 404/457-1172

\section{BALTIMORE DISTAICT}

4756 Trident Court

Baltimore. MD 21227 301/247-2170
CHICACO DISTAICT

B54 Fainway Drive

Bensenville. IL 60106 $312 / 921-6123$

HOUSTON DISTAICT

6610 Hanwin Street

Suite $\# 260$

Houston, TX 77036

$713 / 783-7270$
KANSAS CITY DISTRICT

4000 Somerset Drive

Prairie Village, KS 66208 913/381-9010

LOS ANGELES DISTAICT

14401 Industry Circle

La Mirada. CA 90638

213/868-9926
NEW YOAK DISTAICT

220 Raritan Center Parkway

Edison. NJ 08817

201/225-1555

PITTSBURGH DISTRICT

2247 Babcock Blvd.

Pittsburgh, PA 15237

412/821-6252 


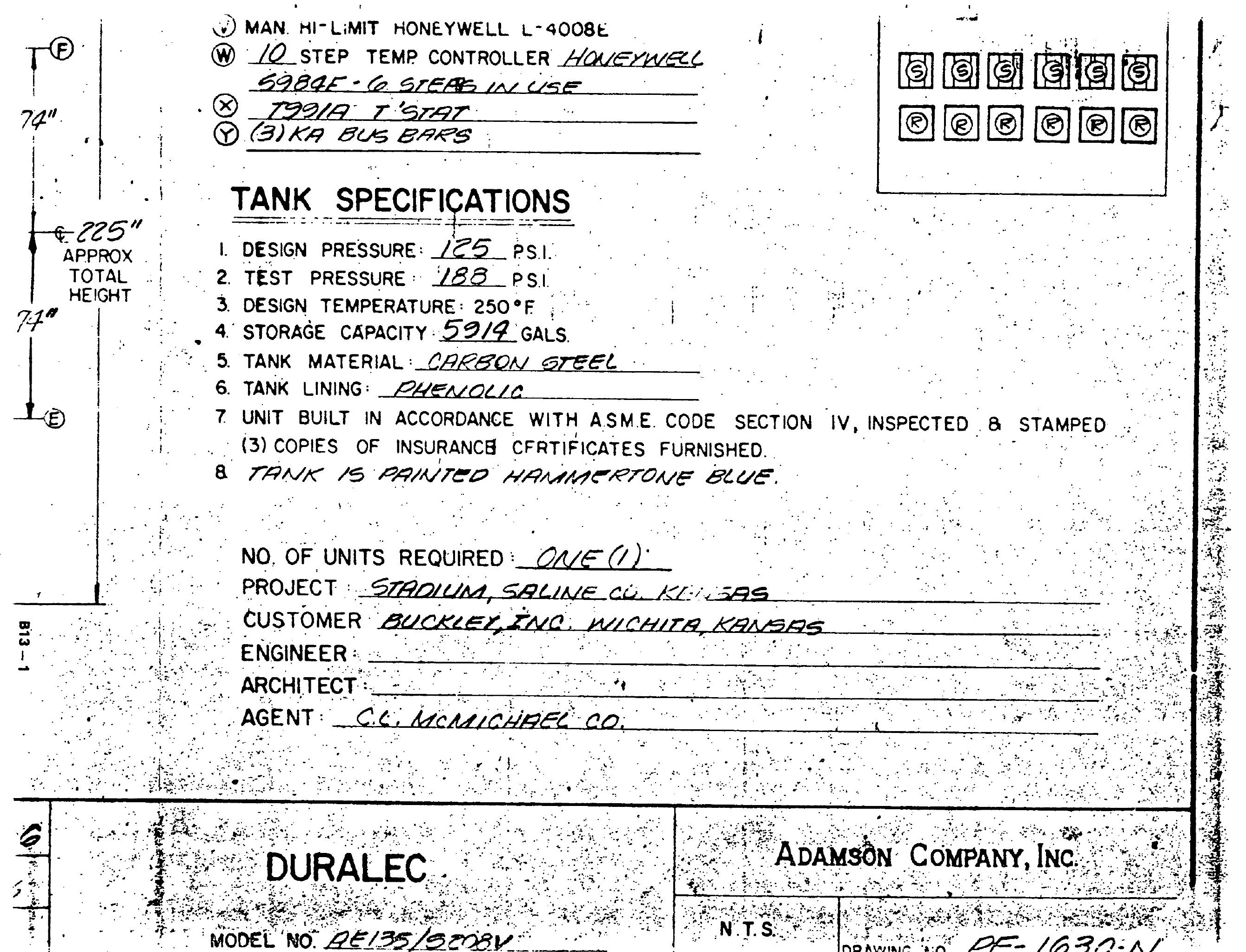


- ELEMENT SPECIFICATIONS

iELECTRIC ELEMENT TO HEAT 553 G.PH OF WATER FROM $90^{\circ}$ F TOLYOF ELEMENT IS RATED @/35K.W.; $180 \mathrm{~V}$, 3 PH. 6QHZ.WI IENO 5 COPPER 'SHEATHED BLADES INDIVIDUALLY REPH ACEABLE A MECHANICALLY FIXED TO A STD. 10" 150"FLANGE 'W/ COPPER FACE CDELTA WIRED CIRCUITS, SO WATT: MAX DENSITY.

FIELD WIRING FOR SUPPLY CONNECTION'S USE 4LQ AREG - WIRE SUITABLE FUR $\left(167^{\circ} \mathrm{F}\right) 75^{\circ} \mathrm{C}$ PER 1975 NEC

FOR GROUNO CONNECTIONS USE NO. 3 ... AWG WIRE.

INTERNAL POWER WIKING TO BE BLACK.

CASH-ACME W WHL-KE

ASME STAMPED TO RELIEVE LOSL OCOBTUMR D LZSPSI.

a 200 F . LTE: NIAES TO

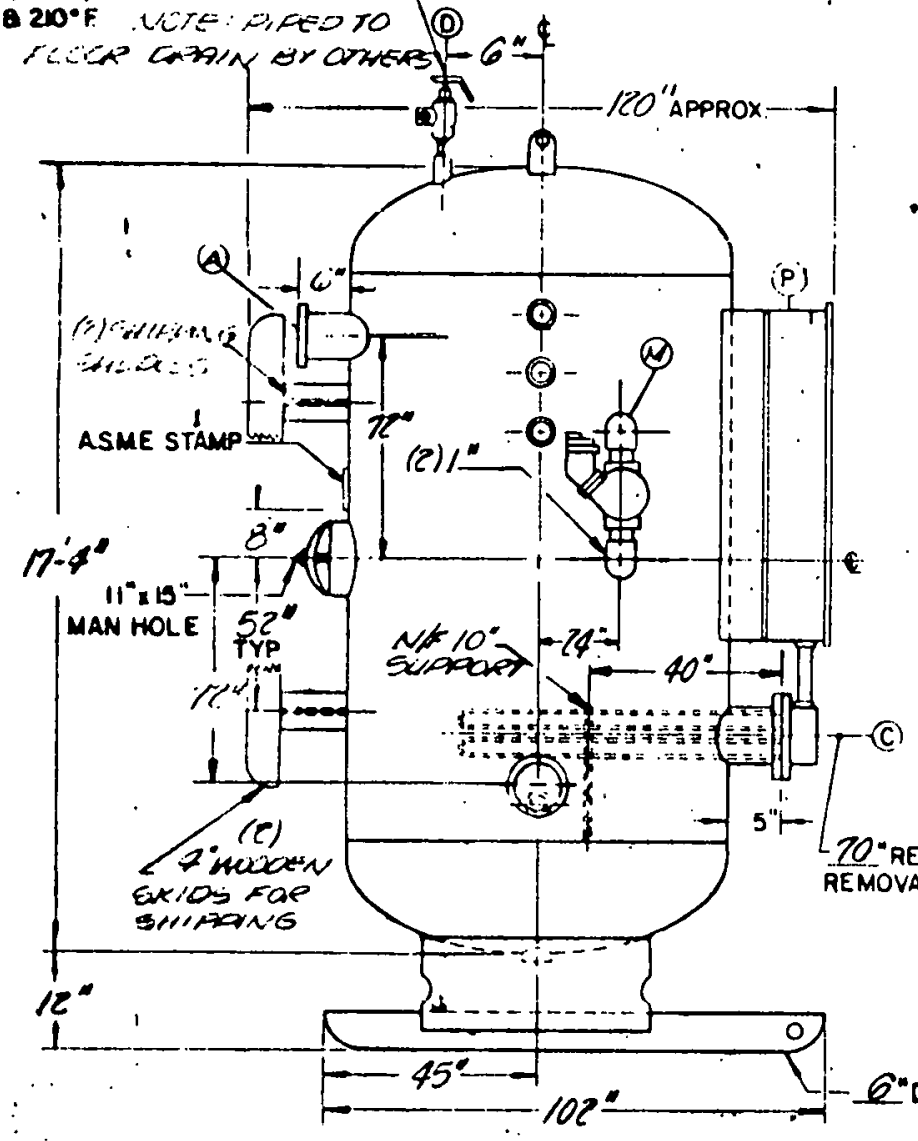

$6^{n} \mathrm{C}$ SND SUPPORTS

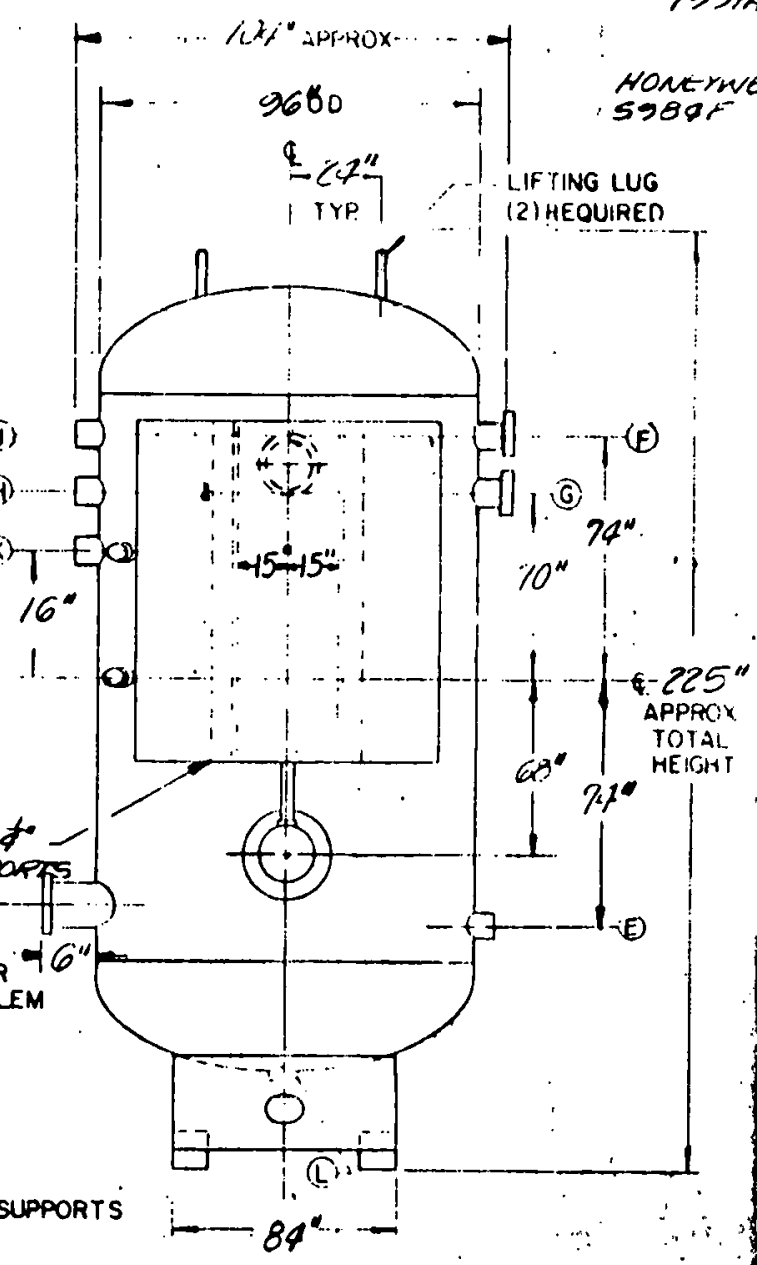

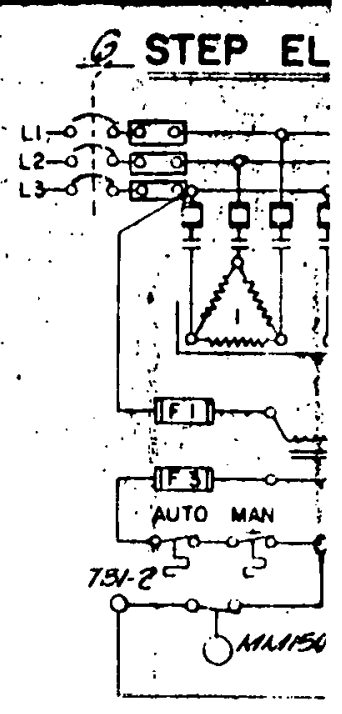

$9 \rightarrow 10$ 


\section{PLUSNUT SPECIFICATIONS COUNTERSUNK HEAD}

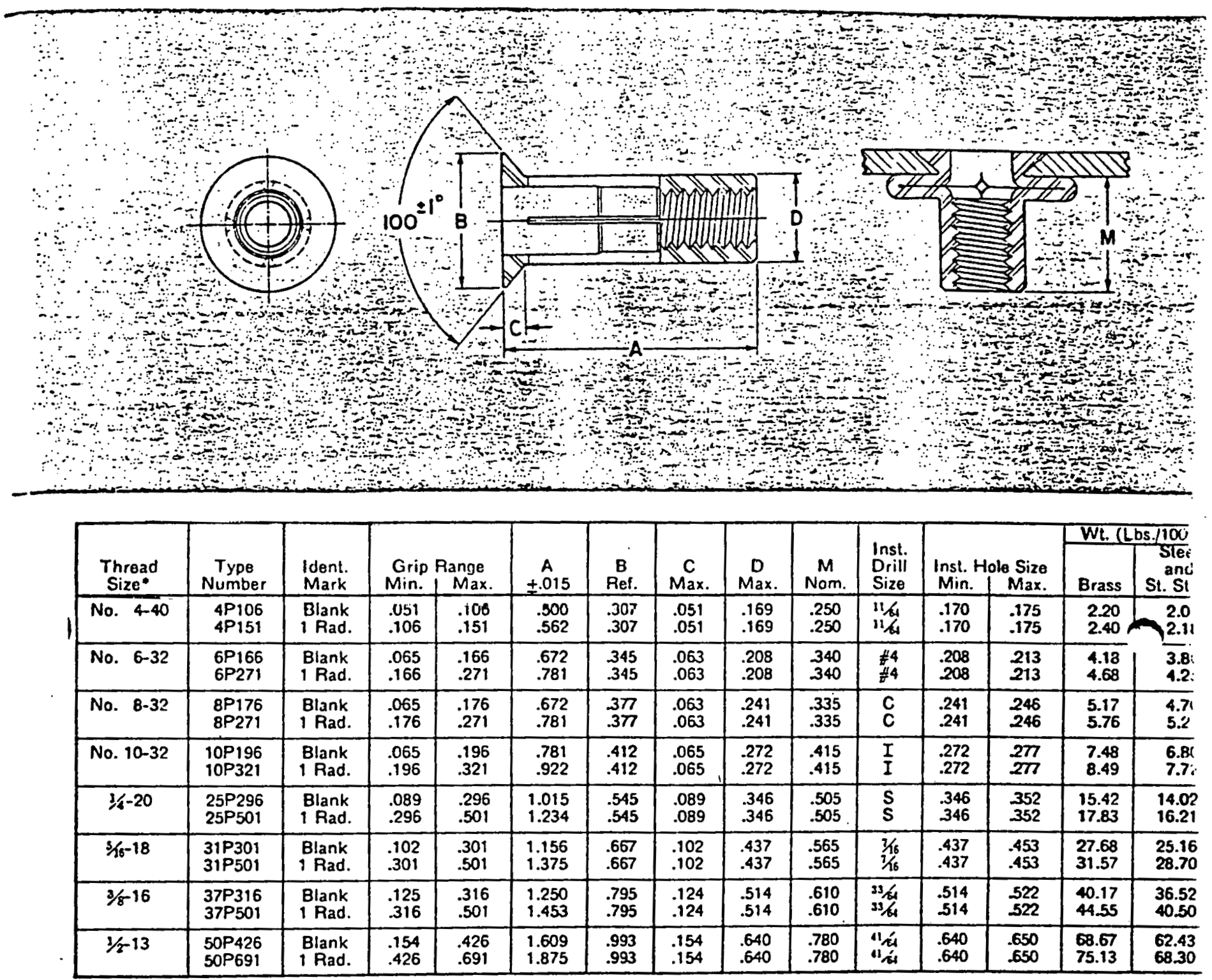

-Both UNC and UNF threads are available in No. 10 and larger sizes.

CODE: Diameter and grip range as tabulated. First lefter of type number indicates material: "S" for stoel, "SS" for stainless steel, and "BR" for brass.

EXAMPLE: S25P296 - Steel $1 / 3-20$ intornal thread $.089-.296$ orip range.

STANDARD PLUSNUT MATERIALS AND FINISHES

\begin{tabular}{|c|c|l|}
\hline Material & Type No. & \multicolumn{1}{|c|}{ Standard Finish } \\
\hline STEEL & C-1108* & $\begin{array}{l}\text { Cadmium Plate - 0002- Minimum } \\
\text { Chickness will meet Foderal Specification: } \\
\text { QQ-P-4166, Class 3, Typo I }\end{array}$ \\
\hline STAINLESS STEEL & 430 & $\begin{array}{l}\text { Pickled and Passivated per Federal } \\
\text { Specification QQ-P-35, Type II }\end{array}$ \\
\hline BRASS & Alloy No. 260 & Bright as machined. \\
\hline
\end{tabular}

- $\mathrm{C}-1108$ and C-1110 steel may be used interchangeably; 
APPENDIX C

SUPPLEMENTAL COST DATA
C - 1 ELECTRICAL EQUIPMENT
C- 2 MECHANICAL EQUIPMENT
C- 3 CONSTRUCTION 


\section{ELECTRICAL EQUIPMENT COMPANY, Inc.}

Electrical Contractors

PHONE 913 827.5249

Salina, Kansas 67401
Residential and Commercial Wiring

P. O. BOX 591

January 9, 1978

Wilson \& Company

P. O. Box 1648

Salina, Kansas

67401

Att: Jim Hall

Re: USD 306

Southeast of Saline

$\mathrm{K}-12$ School Building

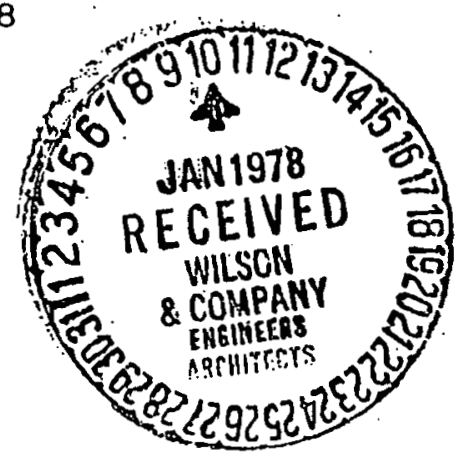

Dear Sir:

We propose to furnish labor and material to complete the electrical work for the Solar Energy installation according to revised drawings showing pump. \#6-8-\&10 as spare pumps only. The starters for pumps $3-4-\& 5$ to be installed in existing motor control center. The starters for pump 7 and 9 to be separate starters. All control iteins furnished by others.

All the above for Eleven Thousand Four Hundred Seventy Five Dollars $(\$ 11,475.65)$.

Sales Tax not included.

Sincerely,

Electrical Equipment Company, Inc.

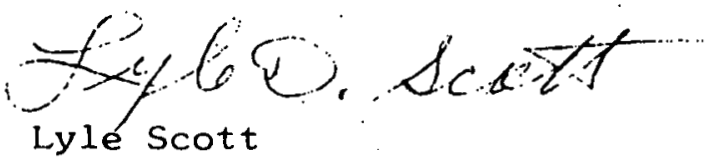

cc: file 
PUCKLEY

INCORPORATED MECHANICAL CONTRACTORS

1600 EAST MURDOCK • P. O. BOX 515 • WICHITA, KANSAS 67201 • PHONE (316) 263.5223

January 9, 1978

Wi1son \& Company, Engineers and Architects

P.O. Box 1648

Salina, Kansas 67401

Nttention: Mr. James M. Ha11. III

Re: Solar Energy System

K-12 School, U.S.D. \#306

Saline County, Kansas

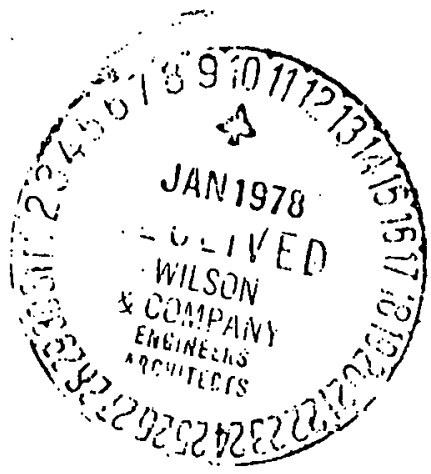

Dcar Jim:

A review of cost parameters of the above referenced project indicates that, based on recent system design modifications as itemized herein, we will be able to hold firm to previously proposed contract dollar amounts; specifically dollar amounts as stated in a written proposal dated March 9, 1977, and a verbal proposal (to modify domestic hot water circulation system) of July 8, 1977. We propose, therefore, to provide a complete mechanical installation as defined in the previously referenced proposals, and modified herein, for the amount of:

ONE HUNDRED SIXTY-THREE THOUSAND SEVEN HUUNDRED and no/100 DOLLARS - $\$ 163,700.00$.

System design modifications include:

1. Location of the solar array approximately one hundred fifty (150) feet southeast of the building location.

2. Installation of solar collectors in twelve (12) banks of twenty-five (25) collectors each.

3. Installation of $1 \frac{1}{2}$ " thick fiberglass insulation on above ground piping; equivalent thickness of urethane foam on below ground piping; aluminum,jacket over insulation exposed to the weather.

4. Installation of Pumps \#P-3 and P-4 in parallel arrangement; installation of Pumps \#P-5, P-7 and P-9 with Pumps \#P-6, P-8 and P-10 provided to the owner as respective stand-by units.

If you have any questions in regard to the above, please advisg.

$\mathrm{MCW} / \mathrm{jm}$

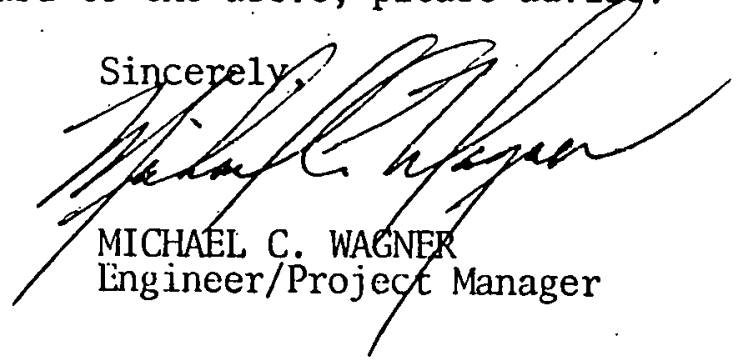

C - 2 
I. S. Trank Gonstruction ba., Iruc.

Seneral bortractors

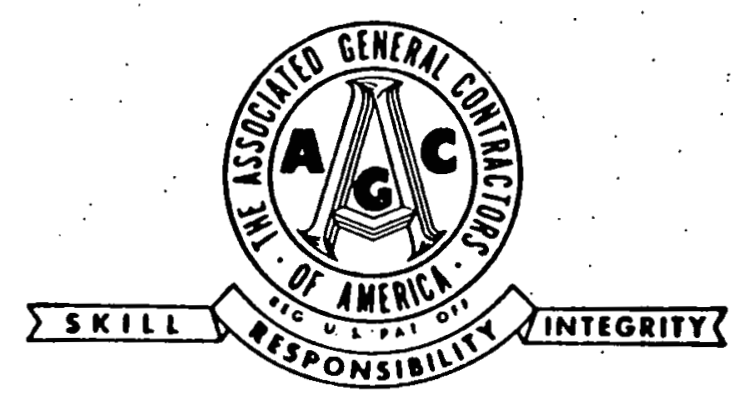

225 SOUTH FIFTH • SALINA, KANSAS 67401

P. O. BOX $X$ PHONE: AC $913827-2760$

1726

January 19, 1978

Mr. James Hal1

Wilson \& Company, Architects

631 E. Crawford

Salina, Kansas 67401

Dear Mr. Hall:

Re Board of Education, Southeast of Saline, Kansas

Unified School District No. 306 (USD 306)

Solar Energy DSE 76-2

We propose to provide the necessary supports for the solar heating units as per drawings to include the concrete bases, angle iron frames, and pipe supports for the mechanical pipe, including painting, for the total sum of $\$ 87,807.00$. (Eighty-Seven Thousand Eight Hundred Seven Dollars).

This proposal subject to review if not accepted within thirty days.

Our breakdown is as follows:

\section{Labor}

1. Excavation

$\$ 10,923$.

2. Concrete

3,040 .

$\$ 5,900$

42,320 .

Subcontract

$-$

3. Structural Steel 16,760

4. Painting - Labor and Material

5. Fence

1,000 .

1,000

$\$ 6,864$

6,864 .

2,000 .

Totalo

$\$ 31,723$.

$\$ 49,220$.

$\$ 6,864$.

$\$ 87,807$.

Sincerety

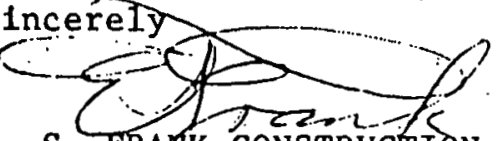

- J. S. FRANK CONSTRUCTION CO., INC.

F. E. Frank, President 


\section{APPENDIX D}

PICTURES

D - 1 PRIMARY \& SECONDARY HEAT EXCHANGER

TANK TO HEAT EXCHANGER PUMP

D-2 COLLECTOR PANEL

LULLECTÓ̈ FIELD

D - 3 PIPING SUPPLY

PIPING RETURN

D - 4 STRUCTURAL FRAMING OF ROWS STRUCTURAL FRAMING REAR VIEW

D - 5 DOUBLE TIERED COLLECTOR ROW COLLECTOR FIELD 


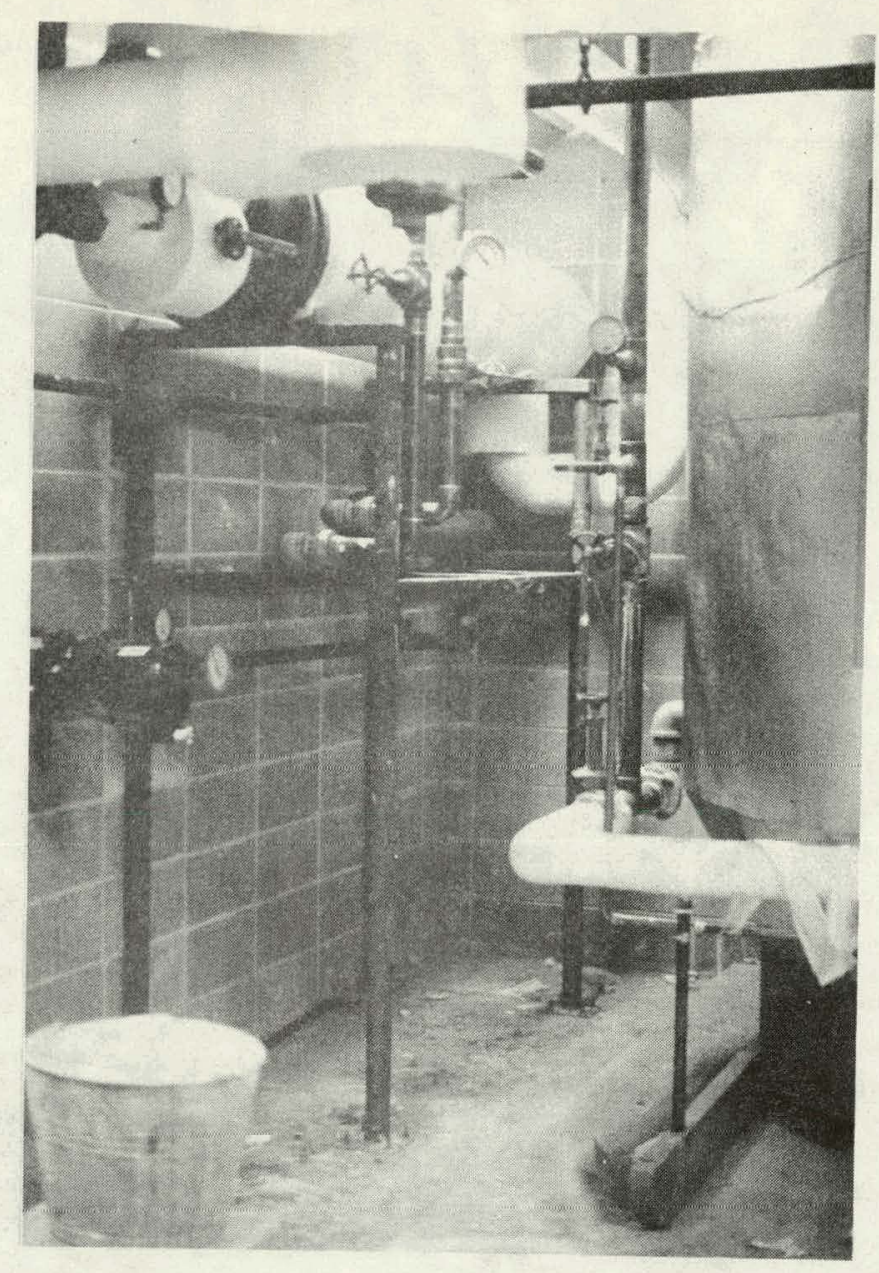

VIEW OF PRIMARY AND DHW HEAT EXCHANGER

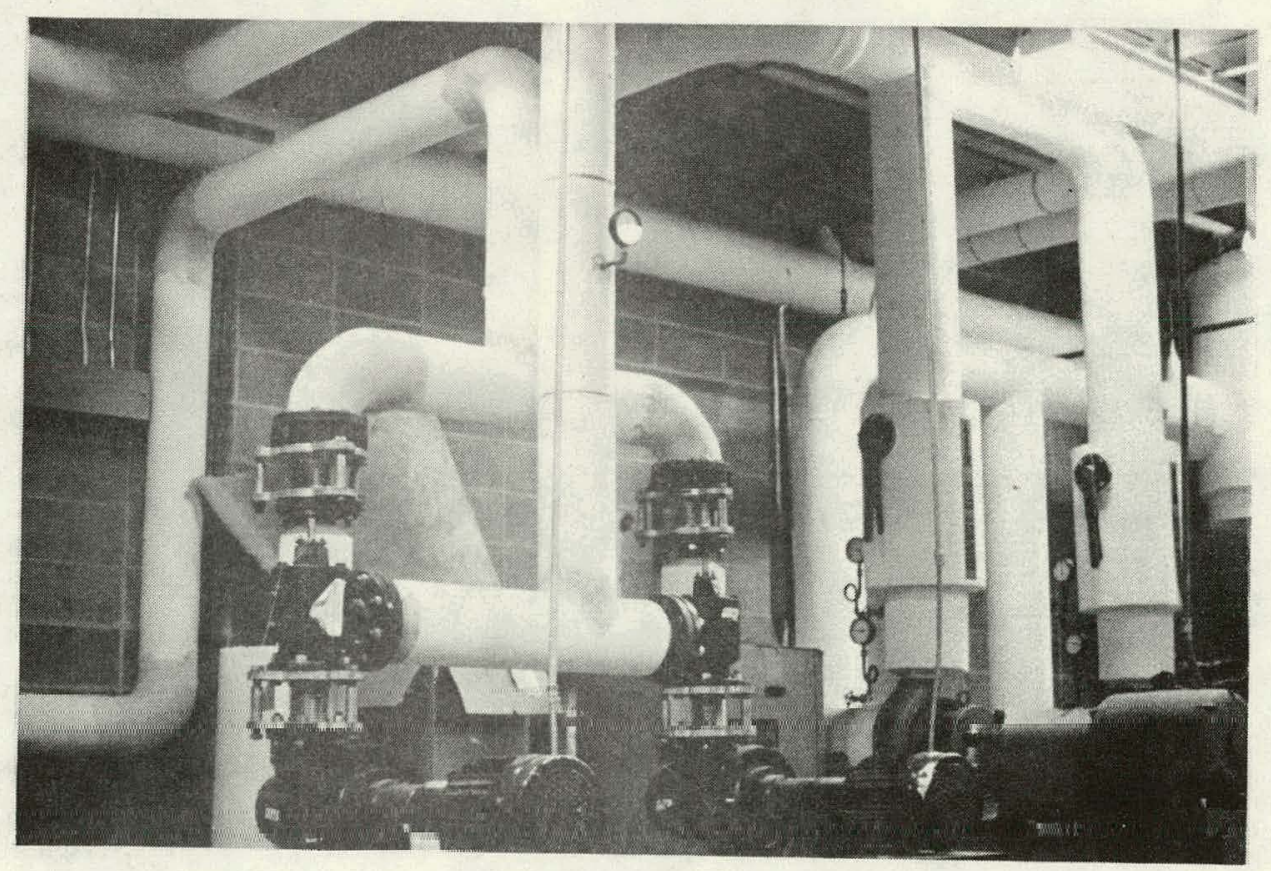




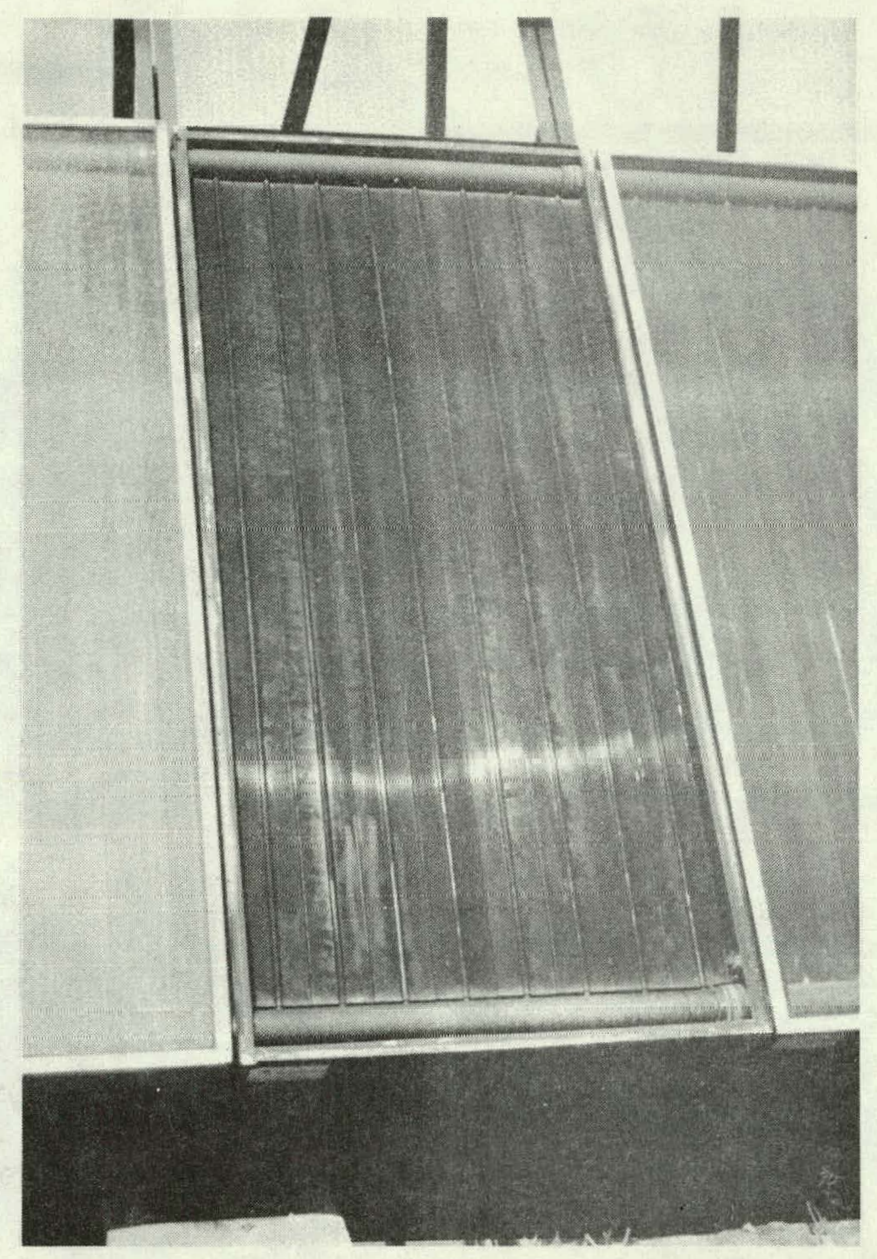

COLLECTOR PANEL WITHOUT GLAZING

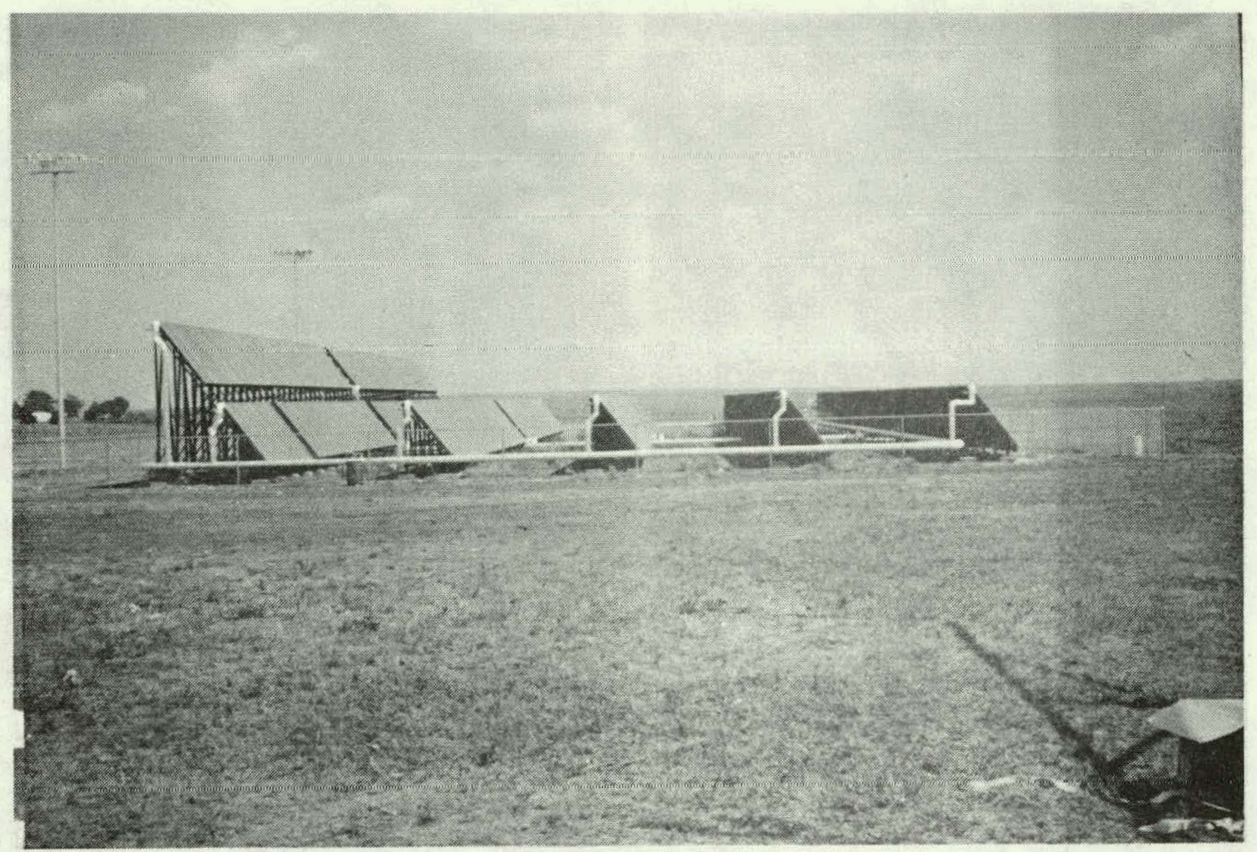

VIEW OF COLLECTOR FIELD 


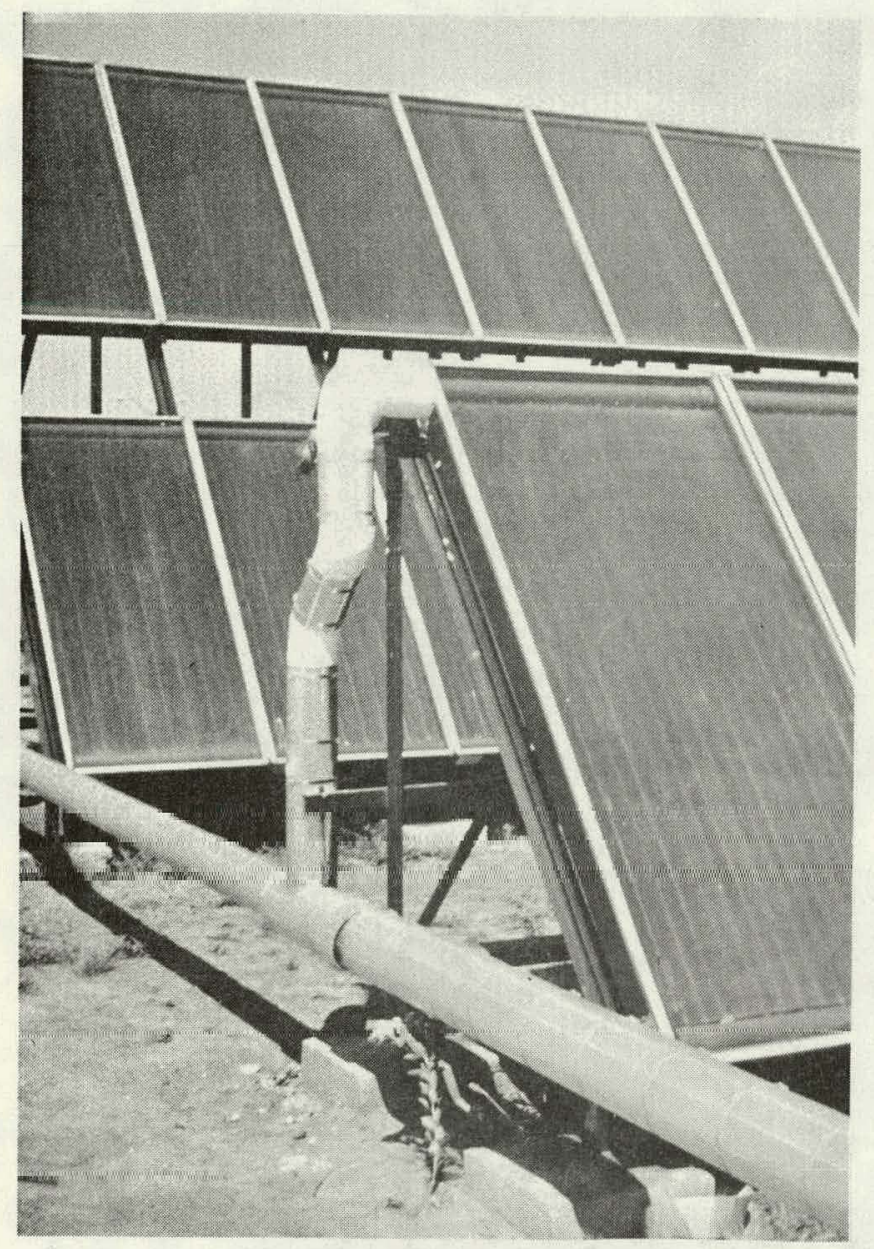

RETURN PIPING

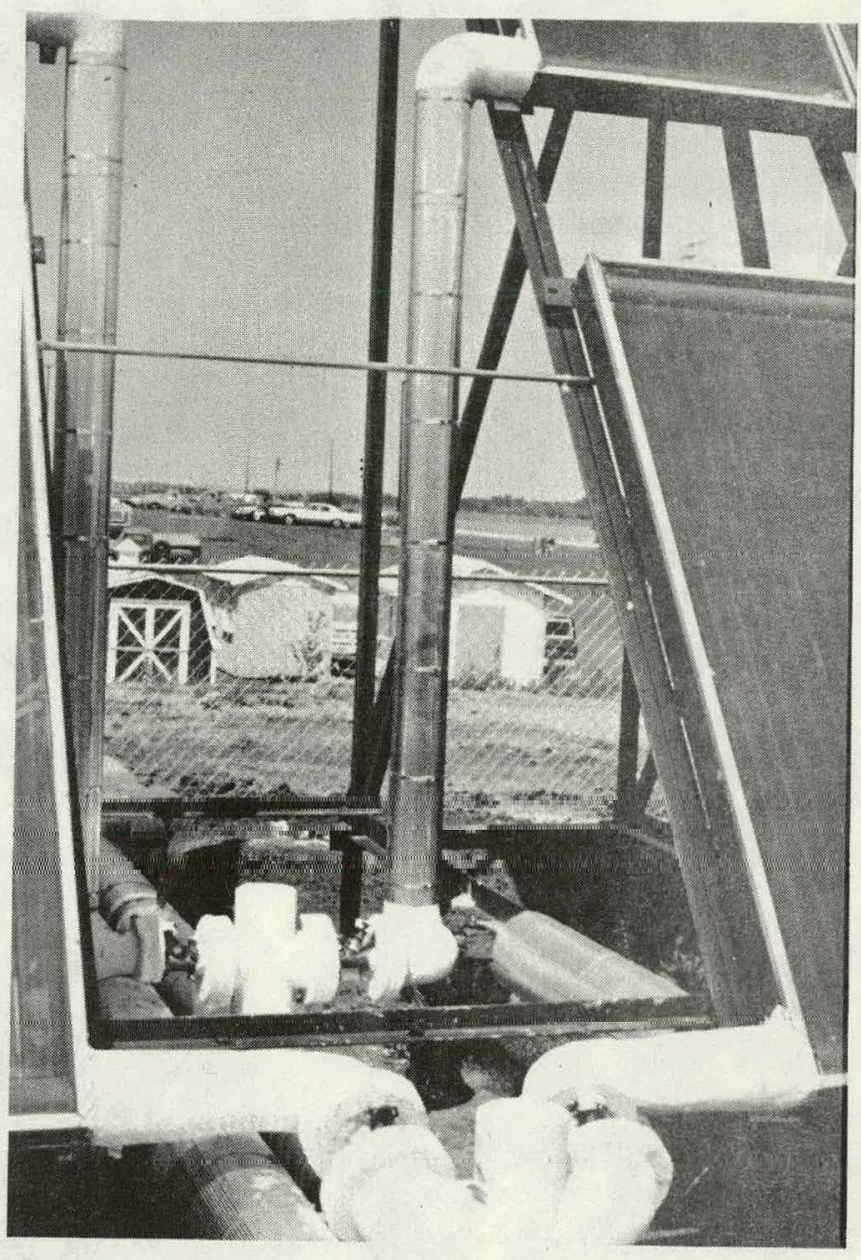

SUPPLY PIPING 


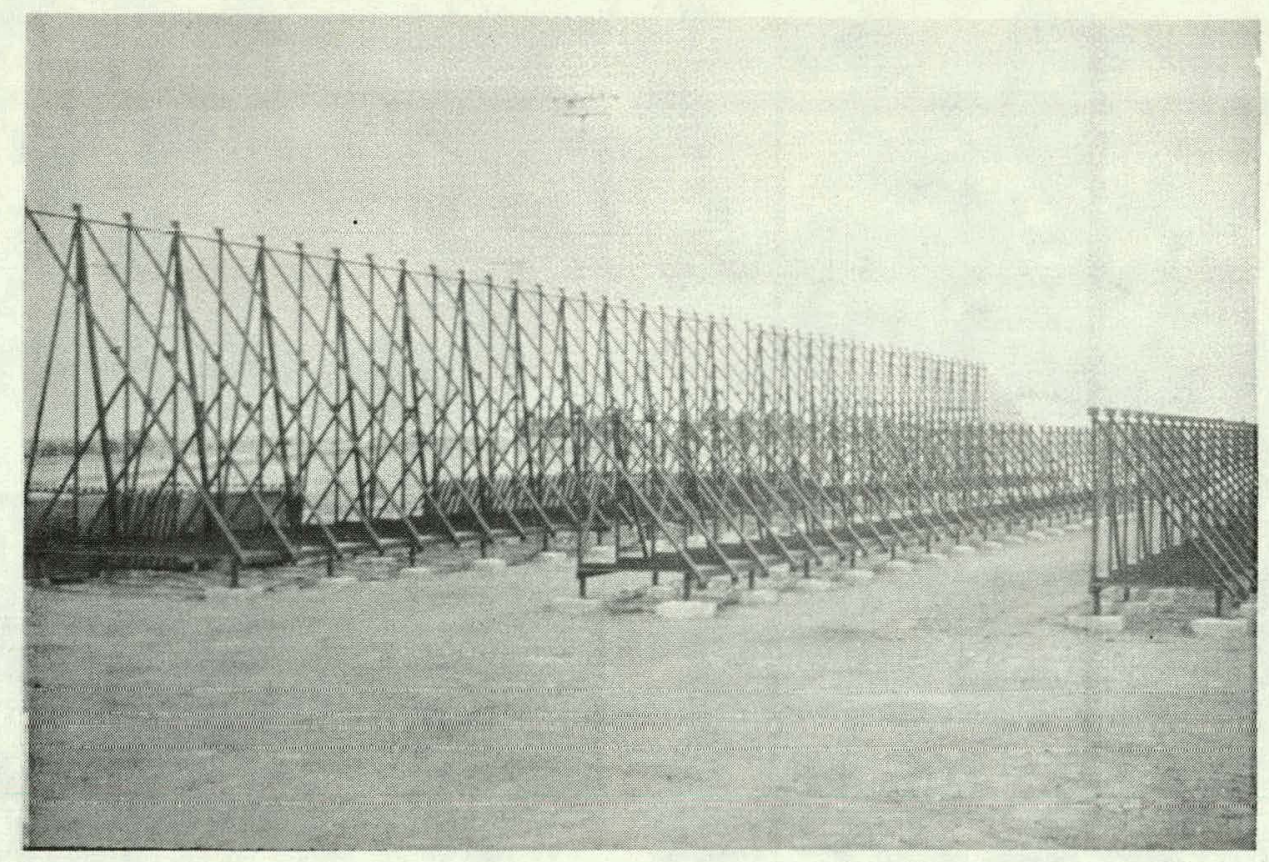

STRUCTURAL FRAMING OF ROWS

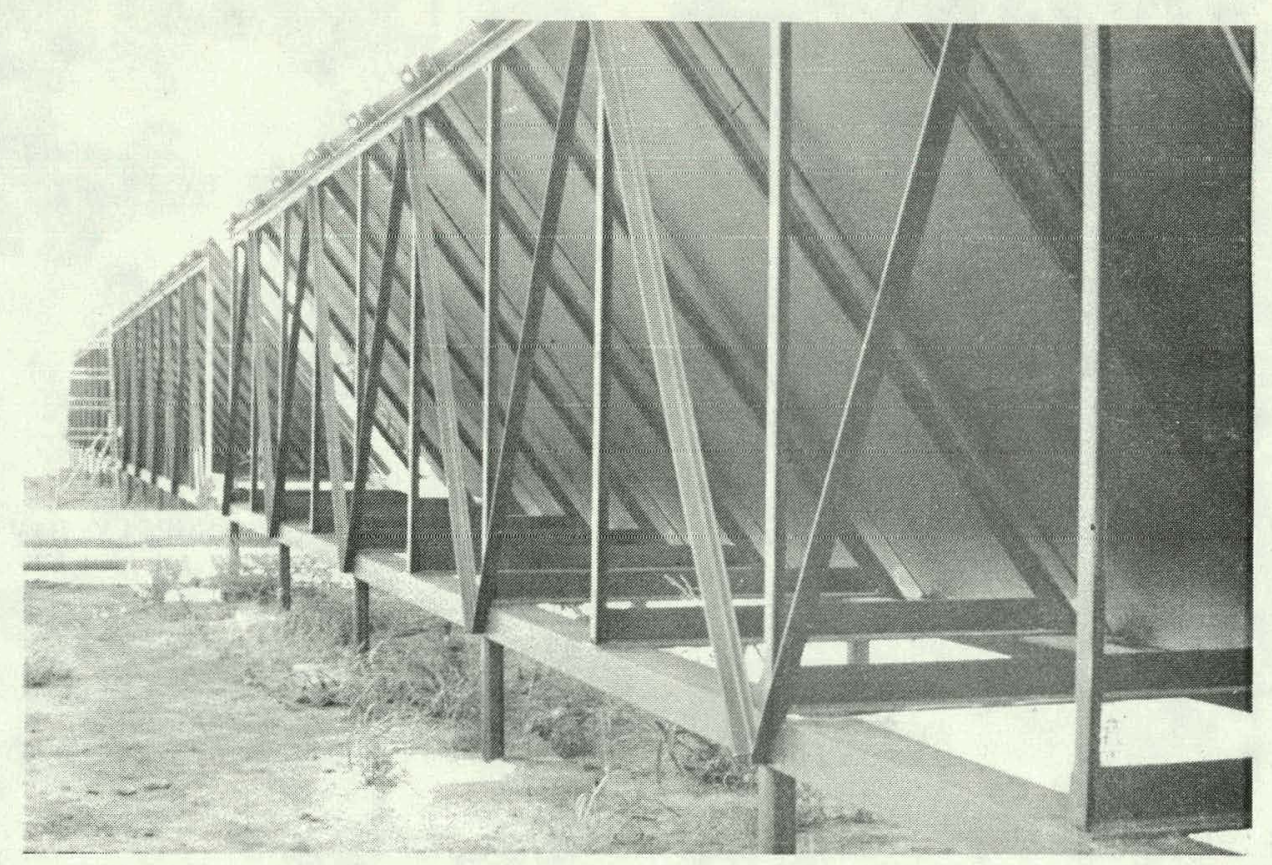

REAR VIEW OF STRUCTURAL FRAMING

D -4 


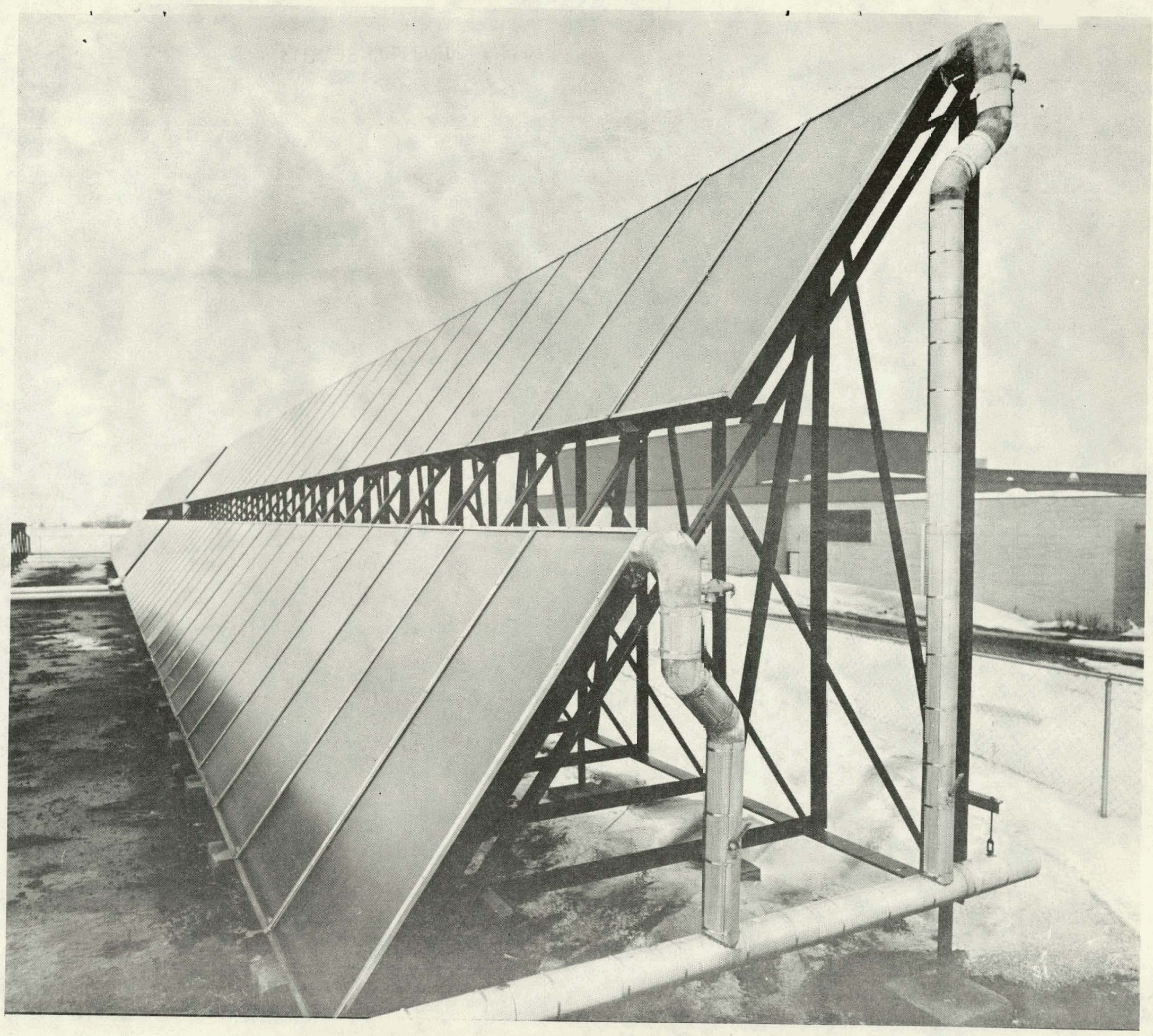




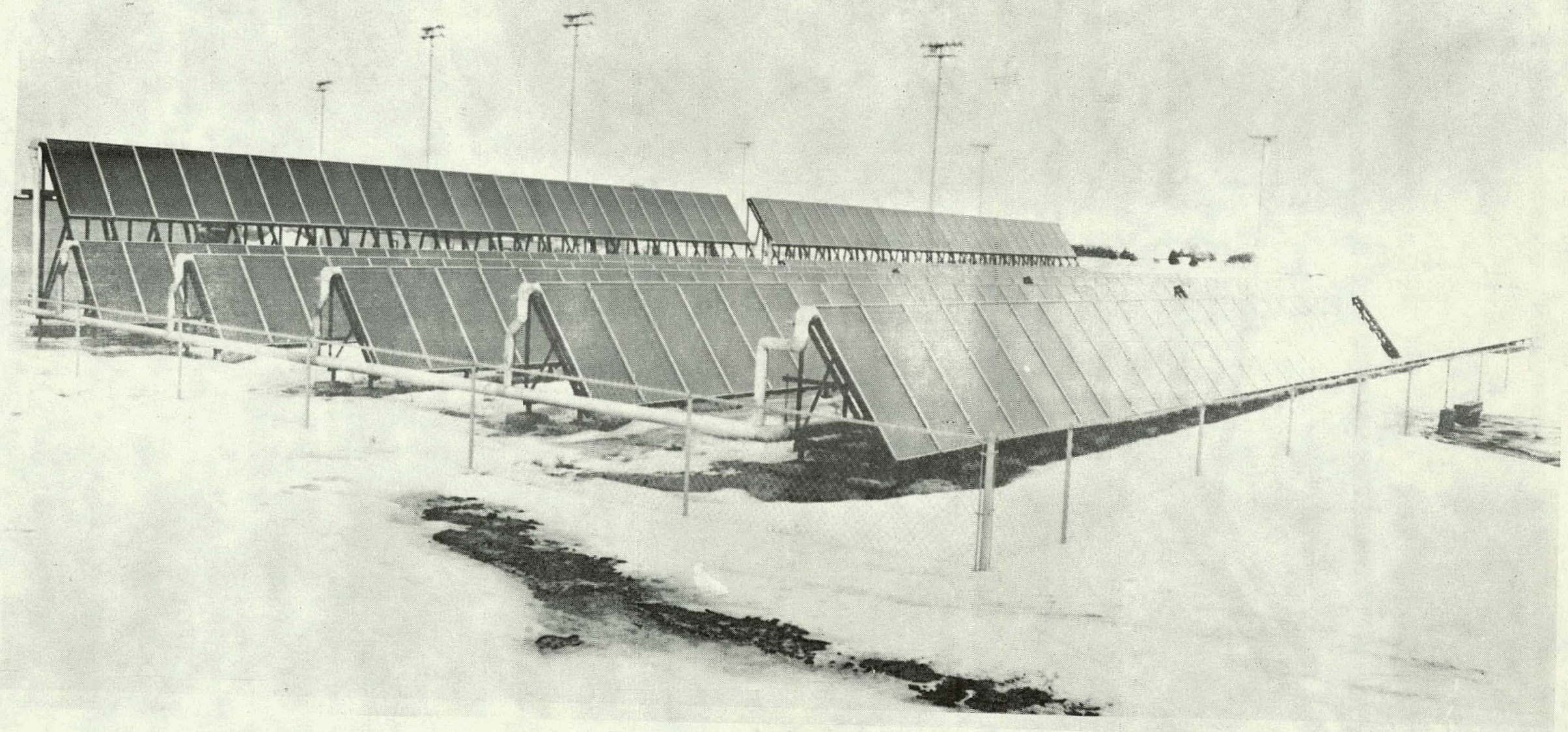

VIEW OF COLLECTOR FIELD 


\section{APPENDIX E}

OPERATIONS AND MAINTENANCE

1. SYSTEM OPERATION SEQUENCE

SYSTEM MAINTENANCE INSTALLATION

2. PUMP - FLUID LOSS

- POWER LOSS

- PROPYLENE GLYCOLL

3. HYDROSTATIC TESTING 


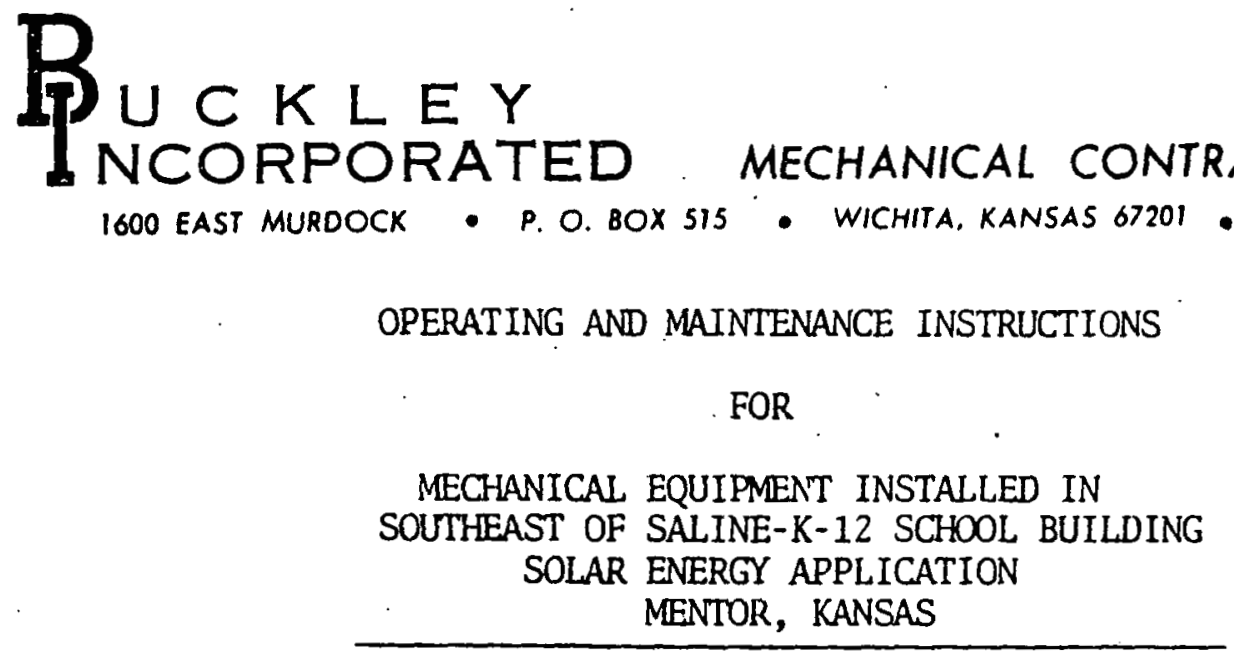

A. System Operation Sequence

(See Contro1 Diagram for Equipment Designations)

1. If inlet water temperature at control " $\mathrm{T}_{1}$ " rises above set point, heat rejection unit fan. will run.

2. When temperature difference between sensor " $\mathrm{S}_{1}$ " in storage tank and sensor " $\mathrm{S}_{3}$ " on collector plate rises above set point on controller " $\mathrm{D}_{1}$ ", pumps \#3 or 4 and 5 will run. When temperature difference falls below set point, pumps will stop. When collector plate sensor " $\mathrm{S}_{5}$ " temperature rises above set point on controller " $D_{3}$ ", pump \#5 will start.

3. When temperature difference between sensor " $S_{2}$ " in domestic hot water tank and sensor " $S_{4}$ " in the storage tank rises above set point on controller " $\mathrm{D}_{3}$ ", pumps \#7 and \#9 will run. When temperature difference falls below set point, pumps will stop. When domestic hot water temperature rises above set point on control "T 3 ", pumps will stop.

B. System Maintenance Installation

1. Service and maintain all mechanical equipment as recommended by Manufacturer.

2. Survey solar collector array daily.

a. Check glass for damage, breakage or excessive dust film; repair, replace or clean as required.

b. Check hose and clamp joints for leaks; should leak exist, tighten clamp as required.

3. Test solar transfer medium (liquid) periodically for proper strength; solution should be approximately $40 \%$ propylene glycol and $60 \%$ water, providing freeze protection to about $-10^{\circ} \mathrm{F}$. Add propylene glycol if necessary; an extra drum of propylene glycol (Dow Chemical "Dowfrost") has been provided for this purpose. 


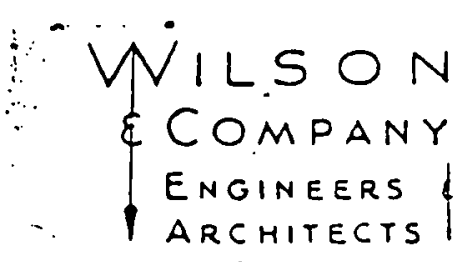

Southeast of Saline, USD 306

RR 1

Gypsum, KS 67448

Aten: Mr. Jim Buxton

Re: USD $306 k-12$ School Building WCEA File: $73-59$

Dear Mr. Buxcon:

In reply to your letter of 4 October 1978 , requesting information regarding the operation of the supplemental solar heating system, we wish to advise you as follows:

1. In case the circulating pump which circulates the antifreeze solution does not function and the relief valves discharge antifreeze solution, we would recommend that you refill the system to replace that fluid which was lost after the circulating pump operation has been restored

2. In case a leak develops in one of the rubber hose connections between pancls we would recommend that you close the values on the inlet and outlet to the panel array which contains the leak and continue to operate the rest of the system in the normal fashion. The fluid concained within that panel array should then be drained and a repair effected. It will be necessary then to refill the panel array which has been drained.

3. In case of a failure of the heat rejector during hot weather or any t1me in the event of a power failure if the system becomes hot, the relief valve's will function to discharge fluid to prevent pressure bulld-up. After the power has been restored, refill the system.

4. After the antifreeze solution has been added, there will not be a freezing problem in either of the panels or in the outdoor piping during the winter. It is important to realize that the antifreeze solution which is used to protect the system from freezing is ixture of 50 percent propylene glycol and water which is an antifre_ze fluid used widely in the food processing industry. It is non-toxic. This fluid should not be confused with ethylene glycol which is commonly used in automobile radiators and is toxic. Under no circumstances should echylene glycol ever be used in this system due to its toxicity. 


\title{
PuCK KLEY $_{\text {NCORPORATED }}$
}

1600 EAST MURDOCK

- P. O. $80 \times 575$

\section{MECHANICAL CONTRACTORS}

\author{
June 11,1979
}

Wilson \& Company

Box 1648

Salina, KS 67401

Attn: George Chitwood

Re: Solar Energy Application

K-12 Schoo1 B1dg., USD \#306

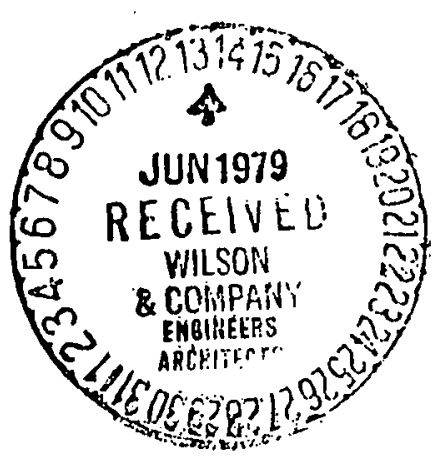

Dear George,

Per your request, the following is a brief description of the system testing procedure lhat our personnel uged on the above réferenced project.

In genera1 - with the exception of solar collector arrays - system piping and components were hydrostatically tested at a pressure equal to that in the water main serving the building (approximately fifty-five (55) psig). This pressure was maintained for a period of twenty-four (24) hours to verify system integrity.

Solar collector arrays were hydrostatically tested at a pressure of approximately thirty (30) psig. It was found that a static test caused the absorbers in the end two or three collectors of each array to "slide" In a direction away from the center of the array; this invariable caused the header tube of at least one collector to slip out of its coupling hose resulting in a leak at that point. To correct this situation, we provided - with the collector Manufacturer's authorization - an internal shim and an external constraint to eliminate the aforementioned movement of the absorber. With the absorber slippage problem corrected, we were able to conduct successful hydrostatic tests at thirty (30) psig for an appropriate period of time.

Should you have any question in regard to the above or require additional information, please advise.

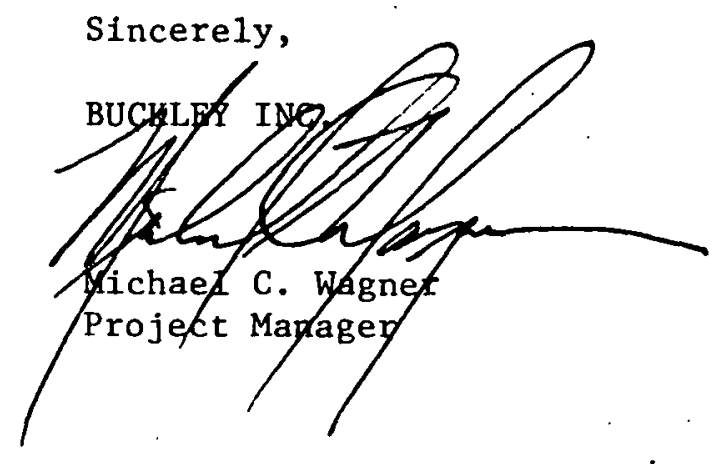

